\title{
Water Soluble Photochromic Fluorescent Nanoprobes based on Diheteroarylethenes and Polymer Coated Quantum Dots
}

\author{
Dissertation \\ for the award of the degree \\ Doctor of Philosophy \\ Ph.D. Division of Mathematics and Natural Sciences \\ of the Georg-August-Universität Göttingen \\ within the doctoral program Grundprogramm Chemie \\ of the Georg-August University School of Science \\ (GAUSS)
}

Submitted by

Sebastián Andrés Díaz

From Buenos Aires, Argentina

Göttingen, 2013 
Thesis Committee

Prof. Dr. Elizabeth Jares-Erijman (Dpto. de Química Orgánica, Universidad de Buenos Aires)

Prof. Dr. Thomas Jovin (Laboratory of Cellular Dynamics, MPI Biophysikalische Chemie)

Prof. Dr. Dietmar Stalke (Inst. for Inorganic Chemistry, Georg-August-Universität Göttingen)

Members of the Examination Board

Reviewer: Prof. Dr. Dietmar Stalke (Inst. for Inorganic Chemistry, Georg-August-Universität Göttingen)

Second Reviewer: Prof. Dr. Thomas Jovin (Laboratory of Cellular Dynamics, MPI Biophysikalische Chemie)

Further members of the Examination Board

Prof. Dr. Tiago Outeiro, (NeuroDegeneration and Restaurative Research, Universitätsmedizin Göttingen)

Jun.-Prof. Dr Guido Clever (Inst. for Inorganic Chemistry, Georg-August-Universität Göttingen)

Prof. Dr. Jörg Enderlein (Drittes Physikalisches Institut, Georg-August- Universität Göttingen)

Prof. Dr. Philipp Vana (Institute of Physical Chemistry, Georg-August- Universität Göttingen)

Date of the oral examination: July $2^{\text {nd }}, 2013$ 




\section{ABSTRACT}

The rapidly growing field of fluorescence microscopy is dependent on the introduction of new, versatile probes. Quantum dots (QDs) are of great interest due to their brightness, fatigue resistance, large extinction coefficient, broad excitation, and narrow emission. In addition, fluorescent probes capable of a systematic modulation of emission intensity provide increased detection sensitivity due to the suppression of background contributions. Of particular interest are probes whose emission can be controlled by external stimuli such as irradiation. By satisfying certain spectroscopic and structural criteria, Förster resonance energy transfer (FRET) can be employed as an efficient means for generating modulation of fluorescence. The use of photochromic molecules, e.g. diheteroarylethenes (PC), as acceptors permits the FRET pair to be reversibly switched between transfer/ nontransfer states with light. This strategy introduced previously as photochromic FRET (pcFRET), was exploited in this thesis to develop photomodulatable fluorescent nanoparticle probes.

A photomodulatable comb-like amphiphilic polymer was synthesized containing lucifer yellow and PC groups as FRET donors and acceptors, respectively. The adopted nano-conformation of the polymer as well as its spectral and switching properties were characterized in organic and aqueous solutions, alone or in combination with a QD template. Self-assembly of the polymer on the surface of a red light emitting QD created a dual-color photoswitchable nanoparticle dispersible in an aqueous medium.

When a $550 \mathrm{~nm}$ emitting QD was coated with a photochromic comb-like amphiphilic polymer it was observed that the polymer conjugated PC molecules self-assembled within the hydrophobic microenvironment demarcated by the outer surface of the semiconductor QD and the polymer cap. This novel surface architecture generated a biocompatible and stable photoswitchable quantum dot (psQD). The psQDs were small ( $\sim 15 \mathrm{~nm}$ diameter) and an emission modulation of up to $50 \%$ in steady state and $47 \%$ in time-resolved fluorescence was observed upon UV irradiation. The psQDs underwent multiple on-off cycles without fatigue in buffer solution with a complete recovery of the initial intensity upon $550 \mathrm{~nm}$ light irradiation. Versions of the psQDs were prepared in which Alexa647 was also conjugated to the polymer and acted as an internal reference, thereby adding ratiometric properties to the construct.

The ensemble psQDs were characterized with a multitude of techniques including singleparticle microscopy. The single particle microscopy supports a model according to which the UV irradiation causes a fraction of the psQDs to enter into a long-lived dark state. Finally psQDs were functionalized by adding biological molecules on the surface, creating targetable psQDs. Preliminary fluorescence microscopy studies were realized on live cells labeled with psQDs. The unique properties of psQDs should lead to numerous applications in cellular imaging. 


\section{Abbreviations}

$\alpha_{\mathrm{PS}}$

A

A431

Abs

ACP

$\mathrm{Al}$

ap

Boc

C12

$\mathrm{CHO}$

$\mathrm{CoA}$

cPC

D

Da

DCC

DLS

E

EDC

EGF

EGFR

eq.

ER

ESIPT

FABP

FLIM

Fmoc

FRAP

FRET

GFP

HDA

HPLC

hv

$J$

$k^{2}$

LY

MWCO

n-BuLi

NMR

NP

OLID

oPC

$\mathrm{p}$
Degree of photoconversion at the photostationary state

FRET acceptor

Epidermoid carcinoma cell line

Absorbance

Acyl Carrier Protein

Alexa Fluor $\mathbb{R} 647$

Anti-parallel

$N$-tert-butoxycarbonyl amine protecting group

n-Dodecylamine

Chinese hamster ovary cell line

Coenzyme A

Closed (photocycled) conformation of diheteroarylethene

FRET donor

Dalton

$N, N^{\prime}$-Dicyclohexylcarbodiimide

Dynamic Light Scattering

FRET efficiency

1-ethyl-3-(3-dimethylaminopropyl)carbodiimide

Epidermal Growth Factor

Epidermal Growth Factor Receptor

Equivalents

Emission Ratio

Excited State Intramolecular Proton Transfer

Fatty-Acid-Binding Proteins

Fluorescence Lifetime Imaging Microscopy

Fluorenylmethyloxycarbonyl carbonate amine protecting group

Fluorescence Recovery After Photobleaching

Förster Resonance Energy Transfer

Green Fluorescent Protein

1-hexadecylamine

High Precision Liquid Chromatography

Light

FRET spectral overlap integral

FRET dipole orientation factor

Lucifer Yellow

Molecular Weight Cut Off

$n$-Butyllithium

Nuclear Magnetic Resonance

Nanoparticle

Optical Lock In Detection

Open conformation of diheteroarylethene

Parallel 
PC

$\mathrm{PCahxNH}_{2}$

$\mathrm{PCalaNH}_{2}$

pcFRET

$\mathrm{PCmNH}_{2}$

$\mathrm{PCmNO}_{2}$

PCmonomer

PEG

PMA

PS

psNP

psQD

PVA

Q

QD

QY

$r_{D A}$

$\mathrm{R}_{\mathrm{f}}$

$\mathrm{R}_{\mathrm{o}}$

ROI

RT

SBB

SE

SmFRET

SOFI

SPM

STED

STORM

$\tau$

TAC

TCSPC

TEM

TIRF

TLC

TOP

TOPO

TR

XPS

Diheteroarylethene or can specifically refer to 3,3'-(3,3,4,4,5,5-hexafluorocyclopent-1-ene-1, 2diyl)bis(2-methylbenzo[b]thiophene)

6-amino-N-(3-(3,3,4,4,5,5-hexafluoro-2-(2-methylbenzo[b]thiophen-3-yl)cyclopent-1-enyl)-2methylbenzo[b]thiophen-6-yl)hexanamide

2-amino-N-(3-(3,3,4,4,5,5-hexafluoro-2-(2-methylbenzo[b]thiophen-3-yl)cyclopent-1-enyl)-2methylbenzo[b]thiophen-6-yl)propanamide

Photochromic FRET

3-(3,3,4,4,5,5-hexafluoro-2-(2-methylbenzo[b]thiophen-3-yl)cyclopent-1-enyl)-2methylbenzo[b]thiophen-6-amine

[3-(3,3,4,4,5,5-Hexafluoro-2-(2-methylbenzo[b]thiophen-3-yl)cyclopent-1-en-1-yl)-2-methyl-6nitrobenzo[b]thiophene]

4-((3-(3,3,4,4,5,5-hexafluoro-2-(2-methylbenzo[b]thiophene-3-yl)cyclopent-1-en-1-yl]-2methylbenzo[b]thiophene-6-yl)amino)-4-oxobutanoic acid

Polyethylene glycol

Poly[isobutylene-alt-maleic anhydride]

Photostationary

Photoswitchable Nanoparticle

Photoswitchable Quantum Dot

Polyvinyl alcohol

Heat

Quantum Dot

Quantum Yield

Donor-Acceptor distance

Retention factor

Förster distance

Region of Interest

Room Temperature

Sodium Borate Buffer

Sensitized Emission

Single Molecule FRET

Superresolution Optical Fluctuation Imaging

Single Particle Microscopy

Stimulated Emission Depletion

Stochastic Optical Reconstruction Microscopy

Fluorescence lifetime

Time-to-Amplitude Converter

Time Correlated Single Photon Counting

Transmision Electron Microscopy

Total Internal Reflection Microscopy

Thin Layer Chromatography

Trioctylphosphine

Trioctylphosphine oxide

Texas Red

X-ray Photoelectron Spectroscopy 


\section{TABLE OF CONTENTS}

Introduction 1

Photochromes 2

Förster Resonance Energy Transfer $\quad 8$

Photochromic Förster Resonance Energy Transfer 11

Nanoparticles 13

$\begin{array}{ll}\text { Scope } & 15\end{array}$

1. Synthesis and Characterization of Diheteroarylethenes and Polymers 21

1.1 Synthesis of Base Diheteroarylethene (PC) 23

1.2 Synthesis of PCs for conjugation to Polymer 25

1.2.1 Synthesis of $\mathrm{PCmNH}_{2}$ and of $\mathrm{PCmonomer} 25$

1.2.2 Characterization of $\mathrm{PCmNH}_{2}$ and of $\mathrm{PCmonomer} \quad 26$

1.3 Photochromes with Extended Functionalizations 30

1.3.1 PC with Primary Amine Terminated Aliphatic Linkers $\quad 30$

1.3.2 Photoactivatable Crosslinking PC 32

1.4 Polymer Synthesis $\quad 32$

1.4.1 Preparation of Photochromic Polymers 32

1.4.2 Polymer Characterization $\quad 34$

1.5 Bifunctional Polymer Synthesis 36

1.5.1 Bifunctionalization with PC and a Fluorescent Dyes 36

1.5.2 Bifunctionalization with PC and Targeting Molecules 38

2. Characterization of Polymer Bound pcFRET Pair 41

2.1 Photoswitchable Polymer in Organic Solvents $\quad 41$

2.1.1 Single Polymer Strands in Solution 43

2.1.2 Polymer Conjugated LY-PC as pcFRET Pair 45

2.1.3 Kinetics of Photoconversion and Fluorescence Quenching of Polymer Chains $\quad 46$

2.2 Photoswitchable Polymer as Polymersomes in Aqueous Solutions 47

$\begin{array}{ll}\text { 2.2.1 Physical Characterization of Polymersomes } & 47\end{array}$

2.2.2 Spectral Characterization of Polymersomes $\quad 47$

2.3 Dual-Color Photoswitchable Nanoparticles $\quad 49$

2.3.1 Physical Characterization of psNP 50

2.3.2 Spectral Characterization of the LY and QD Emission Components of psNP $\quad 50$

2.3.3 psNP as Ratiometric Probes 54

2.3.4 Spectral Kinetics and FRET Systems 55

2.4 Comparisons and Interpretation 57

2.5 Conclusions $\quad 58$ 
3. PHOTOSWITCHABLE QUANTUM DOTS 61

3.1 Photoswitchable Quantum Dot (psQD) 62

3.1.1 Preparation and Purification of psQDs 63

$\begin{array}{ll}\text { 3.1.2 Spectral Characterization of psQDs } & 65\end{array}$

$\begin{array}{ll}\text { 3.1.3 Photoconversion Kinetics } & 67\end{array}$

$\begin{array}{ll}3.2 \text { pcFRET Model for psQDs } & 68\end{array}$

3.2.1 Physical Model Describing the pcFRET System 70

3.3 Dual-color psQDs $\quad 72$

$\begin{array}{ll}\text { 3.3.1 Preparation of Dual-color psQDs } & 72\end{array}$

$\begin{array}{ll}\text { 3.3.2 Spectral Properties of Dual-Color psQDs } & 73\end{array}$

$\begin{array}{ll}\text { 3.3.3 Ratiometric Cycles } & 77\end{array}$

$\begin{array}{ll}\text { 3.3.4 Dual-color psQD Kinetics } & 78\end{array}$

3.3.5 Using Dual-Color psQDs to Study Polymer Coating Stability 79

3.4 psQDs Prepared with Photochromic Polymers Conjugated with Different 81 Linker Length PCs

4. SINGLE PARTICLE MICROSCOPY OF PHOTOSWITCHABLE QUANTUM 87 DOTS

4.1 Imaging psQDs with a Time Correlated Laser Scanning Confocal Microscope $\quad 87$

4.1.1 Sample Preparation $\quad 87$

$\begin{array}{ll}\text { 4.1.2 Experimental Set-up } & 88\end{array}$

4.1.3 Results and Discussion $\quad 88$

4.2 Imaging psQDs with Single Particle TIRF Microscopy 92

4.2.1 Experimental Set-up $\quad 92$

$\begin{array}{ll}\text { 4.2.2 Data Analysis } & 92\end{array}$

4.2.3 Analysis of Open and PS State Measurements 93

$\begin{array}{ll}\text { 4.2.4 Photoswitching experiments } & 97\end{array}$

$\begin{array}{ll}\text { 4.2.5 Secondary Observations } & 97\end{array}$

$\begin{array}{ll}4.3 \text { Conclusions } & 98\end{array}$ 
5. BIOLOGICAL TARGETING OF PHOTOSWITCHABLE QUANTUM DOTS 101

5.1 psQDs Targeted Through Post-Coating Functionalization 102

$\begin{array}{ll}\text { 5.1.1 Conjugation of Texas Red Cadaverine to psQDs } & 103\end{array}$

5.1.2 Conjugation of Epidermal Growth Factor to psQDs 104

$\begin{array}{ll}\text { 5.1.3 Conjugation of Streptavidin to psQDs } & 105\end{array}$

$\begin{array}{ll}\text { 5.1.4 Conjugation of Biotin to psQDs } & 106\end{array}$

5.2 Fluorescence Imaging Experiments with Post-Coating Functionalized psQDs $\quad 107$

5.3 psQDs Targeted Through Functionalized Polymer Coating 111

$\begin{array}{ll}\text { 5.3.1 Photophysical and Surface Characterization } & 111\end{array}$

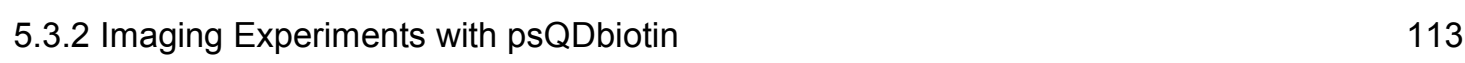

$\begin{array}{ll}5.4 \text { Conclusions } & 114\end{array}$

6. SUMMARY \& OUTLOOK 117

$\begin{array}{ll}\text { Chemistry } & 117\end{array}$

$\begin{array}{ll}\text { Nano-Conformations } & 118\end{array}$

$\begin{array}{ll}\text { Photoswitchable Quantum Dots } & 119\end{array}$

$\begin{array}{ll}\text { Outlook } & 120\end{array}$

7. EXPERIMENTAL 123

$\begin{array}{ll}7.1 \text { Synthesis } & 123\end{array}$

$\begin{array}{ll}7.2 \text { Measurements } & 130\end{array}$

$\begin{array}{ll}7.3 \text { Procedures } & 131\end{array}$

$\begin{array}{ll}\text { Acknowledgements } & 135\end{array}$ 


\section{INTRODUCTION}

Technological breakthroughs in microscopy have pushed the limits of what is detectable in cell biology beyond what was once thought physically possible. Faster and more sensitive camaras, precise piezoelectronics, and improved software, along with conceptual breakthroughs such as STED (Stimulated Emission Depletion Microscopy), ${ }^{1}$ SOFI (Superresolution Optical Fluctuation Imaging), ${ }^{2}$ and STORM (Stochastic Optical Reconstruction Microscopy) ${ }^{3}$ have pushed fluorescence microscopy to new heights. A clear example is how super-resolution techniques have overcome the Abbe diffraction limit, which states a lower limit of $200 \mathrm{~nm}$ resolution for light microscopy, with observed details as small as $20 \mathrm{~nm}^{4}$ As the conventional theoretical limits are overcome it has become clear that one current limitation is the development of new fluorescent probes. A recent editorial in Nature focused on this topic and provides an illuminating quote: Although advances in instrumentation and informatics should not be overlooked, many researchers believe that it is better-performing fluorescent labels that will allow super-resolution microscopy to continue to move forward. "That's an area where the field will see the biggest advances, ... That's been limiting all of us. "5 The desired properties of these advanced fluorescent probes include: brightness, fatigue resistance, large extinction coefficients, high quantum yields, and the possibility of modulating the fluorescence. Semiconductor nanocrystals, or Quantum Dots (QDs), are particularly well suited due to their brightness, fatigue resistance, and large extinction coefficients while also possessing broad excitation and narrow emission. The utilization of a photochromic molecule as an acceptor for Förster Resonance Energy Transfer (FRET) allows for controlled photomodulation of the QD emission. ${ }^{6}{ }^{7}$ For biological applications the QD and the photochromic acceptor, along with the advantageous properties they present, must be made water soluble without drastically affecting the quantum yield of the QD emission and photochrome transformation. My work focused on creating a new fluorescent probe that presented many of these desired properties by combining photochromism, FRET, and QDs. Therefore a short introduction on these three topics will follow. 


\section{Photochromes}

Photochromic compounds are characterized by a reversible transformation that occurs when they are illuminated at appropriate wavelengths. ${ }^{8}$ The transition is between two different structural forms with distinct absorption spectra. It is common to find that physical properties such as solubility, viscosity, refractive index, and dielectric constants change along with the absorption spectra.
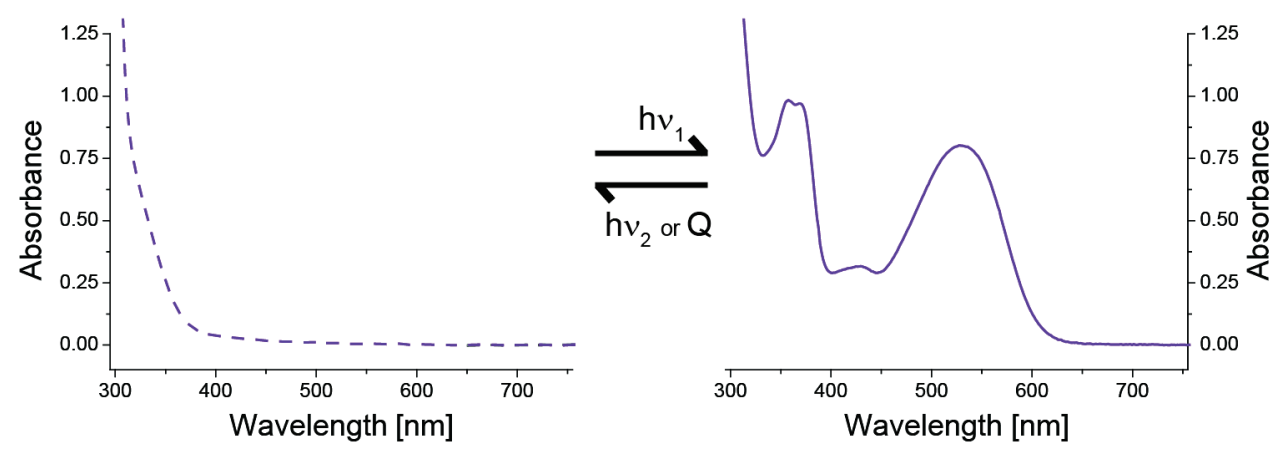

Figure 1: Spectral change of an example photochromic molecule upon irradiation.

Dr. Willy Marckwald in the 1890's was the first to correctly describe the phenomenon, while the term photochromism was coined by Dr. Yehuda Hirshberg in the 1950's while working with spiropyrans. ${ }^{9}$ For a molecule to be photochromic the reaction must be reversible, and this reversal can occur through a thermal mechanism, irradiation with a different wavelength, or both. When the photoreaction can proceed along one energy surface, the activation energy being a small enough barrier, then the thermal mechanism will be a competing pathway. In Scheme 1 a selection of the large set of known photochromic molecule families are presented. The first molecules present thermal reversion (marked as Q, heat) while the last two are examples of thermally stable photochromes at room temperature. Thermal reversion can be beneficial or detrimental depending on your selected use of the photochrome. In applications such as holographic materials the speed of both the conversion and the thermal reversal is paramount, and recent examples have demonstrated films with millisecond discoloration. ${ }^{10}$ A time resolution that is comparable with that of the human eye. In other applications, such as the fluorescence modulation studied in this thesis, the lack of thermal reversion permits long lived conformations and a greater control of the fluorescence emission. Photochromic systems have found applications in multiple academic areas as well as many successful commercial products. 
Azobenzene
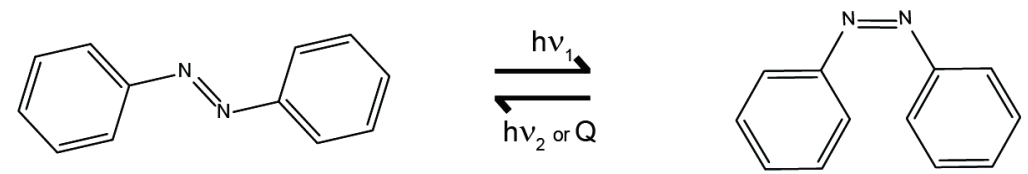

Spiropyrans
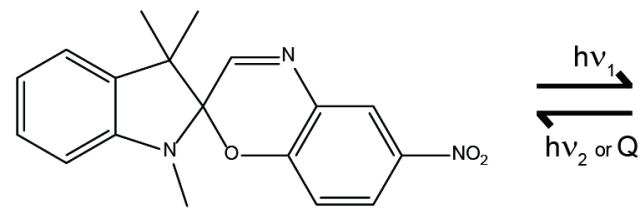<smiles>C[N+]1=C(/C=N/c2cc([N+](=O)[O-])ccc2Cl)C(C)(C)c2ccccc21</smiles><smiles>C1=CC(C2(c3ccccc3)C=Cc3c(ccc4ccccc34)O2)=CCC1</smiles>

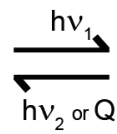<smiles>O=C1C=Cc2ccccc2/C1=C/C=C(c1ccccc1)c1ccccc1</smiles>

Fulgides<smiles>CC1=C2C(=O)OC(=O)C2=C(C)c2cc(C)oc2C1</smiles>

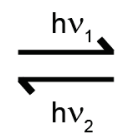<smiles>CC1=CC2=C(C)C3=C(C(=O)OC3=O)C(C)(C)C2(C)O1</smiles>
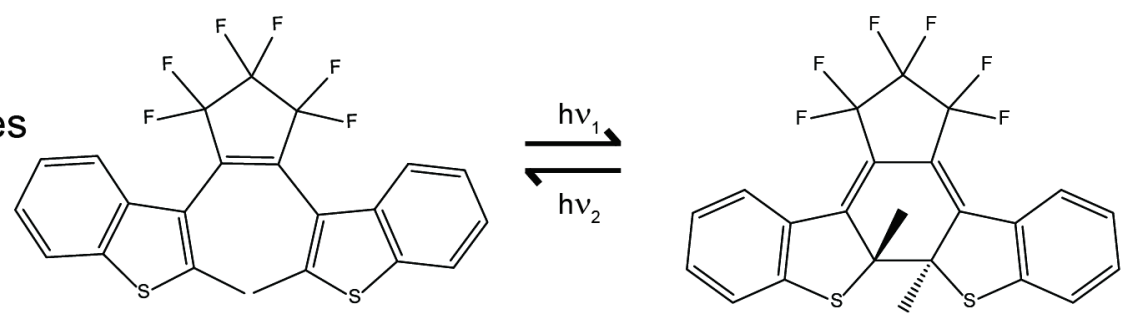

Scheme 1: Families of photochromic molecules. Fulgides and Diheteroarylethenes do not undergo thermal reversion.

The field of data storage has long been interested in photochromic materials. Photochromic materials are erasable, rewritable, and store data in an optical manner (instead of magnetic). ${ }^{11}$ The optical mode of data storage allows for higher data density and even more important is the possibility of 3D storage, since todays traditional 2D media (i.e. DVDs and Blu-ray) have reached their limit. ${ }^{12}$ The first demonstration of a 3D optical memory was demonstrated by Peter Rentzepis and collaborators using spirobenzopyrans and a two-photon process for writing as well as reading and erasing. ${ }^{13}$ 


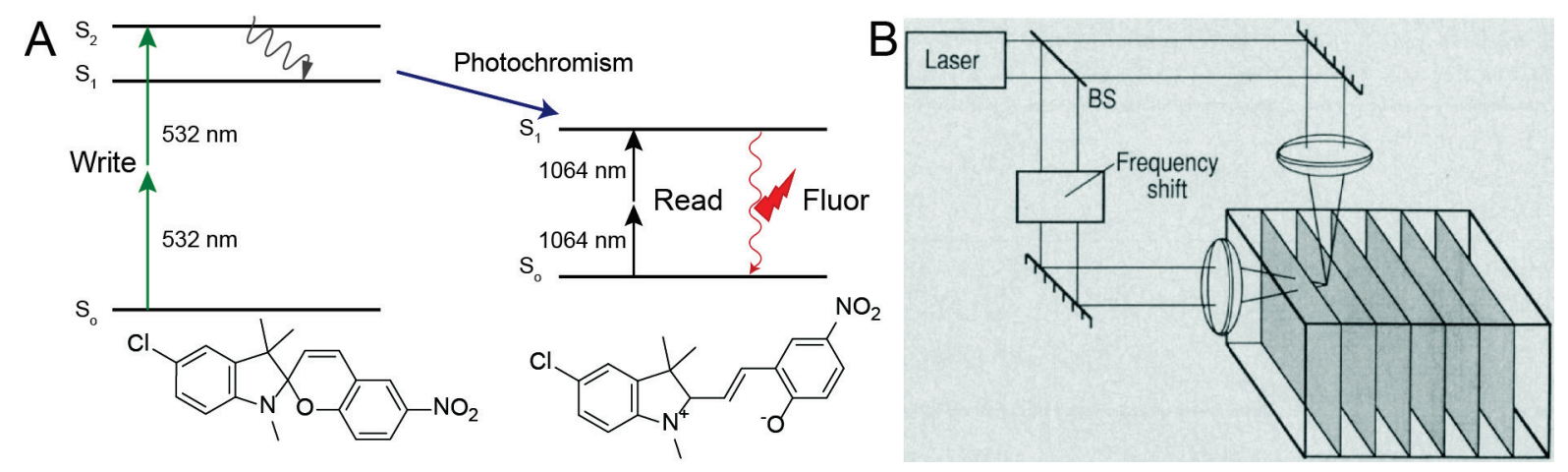

Scheme 2: A) Two-photon read-write process using spirobenzopyran energy levels. B) Scheme of two-photon 3D data storage scheme. ${ }^{13}$ C AAAS 1989.

Optical information can also have multiplexed read-outs such as wavelength, polarization or phase. Uchida et $\mathrm{al}^{14}$ demonstrated a recording system that presented an 8 -state read-out by combining 3 photochromic moieties in one polymer; more importantly each state could be read in the IR region in a nondestructive manner. In another example of multiplexing, diheteroarylethenes polymer films were used to create read-outs that combine spectral as well as polarization and angular multiplexing. ${ }^{15}$
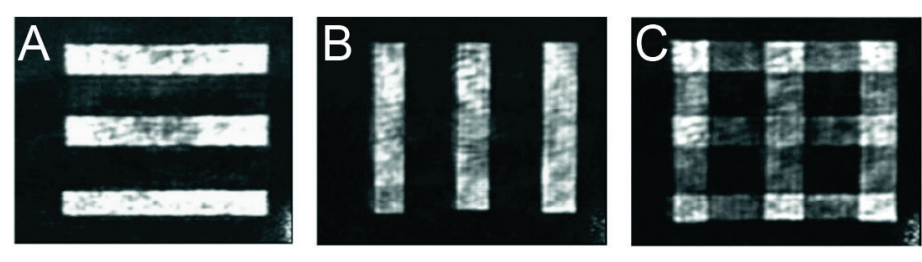

Figure 2: Retrieved images from the polarization multiplexing holograms. (a) Parallel circular polarization hologram at $0^{\circ}$ (B) Orthogonally circular polarization hologram at $90^{\circ}$ (C) Overlapping retrieved image at $45^{\circ}$. (C) SPIE 2008.

To this date there is no commercial availability of 3D optical data storage, though multiple companies are exploring the technology and it may be just over the horizon.

The use of photochromic molecules in supramolecular chemistry is extensive and complex. Even a basic review exceeds the scope of this introduction, so it will be limited to a few examples in two key areas. The first area is the host-guest interaction, where traditionally the photochrome induces a morphological change which modulates the interaction. Han et al ${ }^{16}$ demonstrated the selective encapsulation of $\left[\mathrm{B}_{12} \mathrm{~F}_{12}\right]^{-2}$ ions by creating a photoswitchable cage based on the rigid/flexible modulations of dithienylethene. 
A)

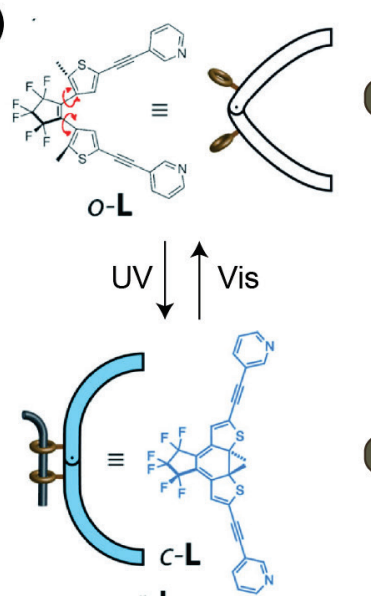

B)
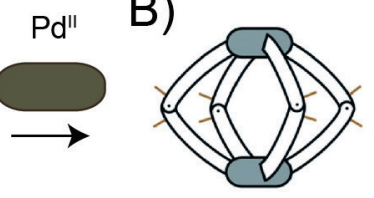

UV $\downarrow \uparrow$ Vis

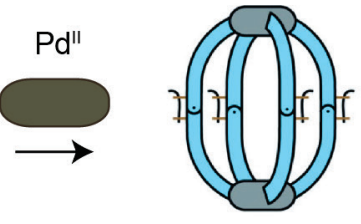

C)
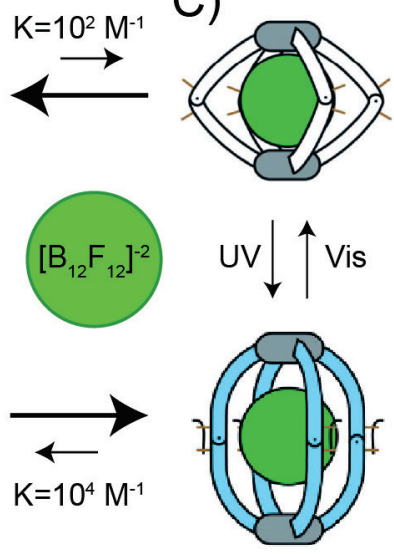

Scheme 3: A) The conformationally flexible bis-monodentate pyridyl ligand based on a dithienylethene photoswitch is converted into its rigid closed isomer c-L upon irradiation at $365 \mathrm{~nm}$. The process can be reversed by white light. B) Addition of stoichiometric amounts of $\mathrm{Pd}^{\mathrm{II}}$ leads to quantitative formation of coordination cages $\mathrm{C}$ ) Both cage isomers can encapsulate the spherical guest to varying degrees. Irradiation of the host-guest complexes results in the reversible uptake and release of the guest. (C) Wiley-VCH 2013. Adapted from ${ }^{16}$.

The lab of Akira Harada has done extensive work on polymers and gels that are functionalized with cyclodextrins and azobenzenes as host/guest pairs. In fact they have been able to apply this property in the macroscale, creating milimeter sized gels that bind together but dissociate upon UV irradiation. $^{17}$
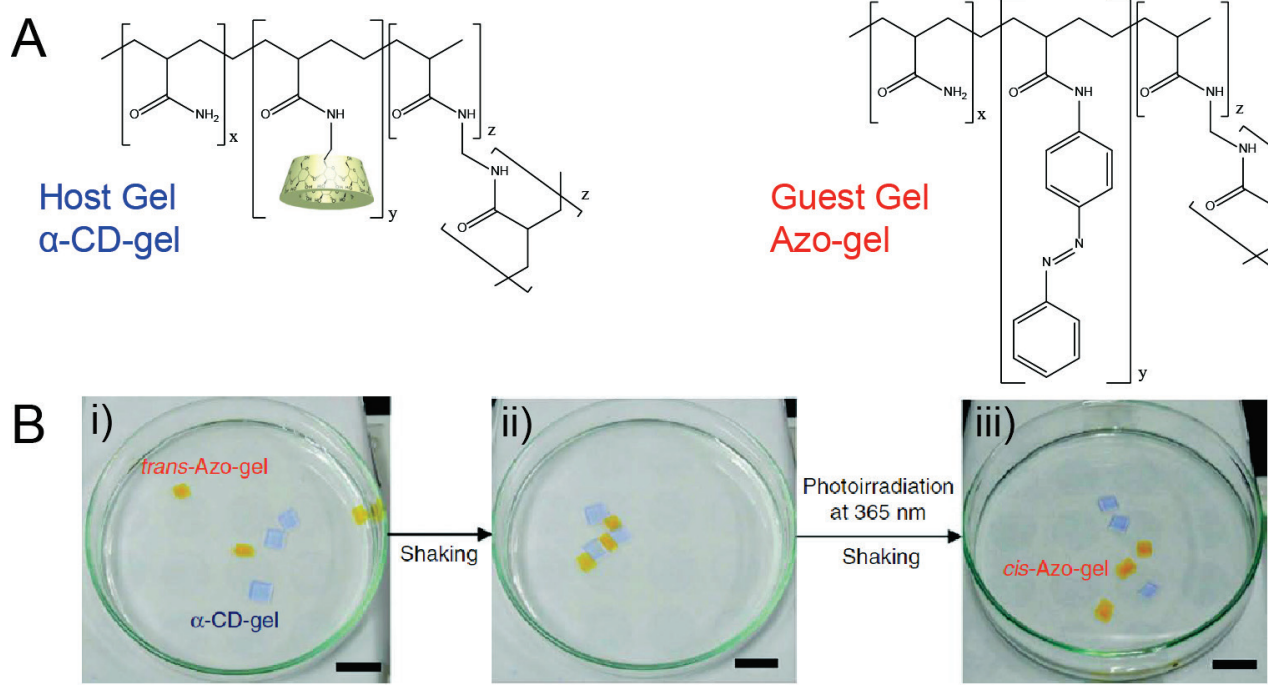

Figure 3: (A) The chemical structures of a host gel ( $\alpha$-CD-gel) and guest gel (Azo-gel) (B) Gel assembly of $\alpha$ $\mathrm{CD}$ gel (blue) with the trans-Azo-gel (orange), and gel dissociation with irradiation with ultraviolet light. i) Addition of water to the gels in a Petri dish. ii) Agitation of the gels for several minutes led to the assembly of an $\alpha-C D$-gel/Azo-gel. iii) Photoirradiation at $365 \mathrm{~nm}$ led to the dissociation of gels. ${ }^{17}$

A second supramolecular chemistry area in which photochromes are present is photomodification to create two distinct supramolecular structures. The Feringa lab has recently observed in real-time the light driven disassembly of nanotubes prepared from amphiphilic molecules containing a photochromic core. ${ }^{18}$ The tubes are bilayers of photoresponsive bis-thiaxanthylidenes core with PEG (polyethylene glycols) and alkyl chain groups that self-assemble upon addition to 
water. Upon UV irradiation the tubes are shifted to aggregates as the rigidity in the photoresponsive bis-thiaxanthylidenes inhibits the tube formation. A more subtle demonstration was made by Li et al ${ }^{19}$ in their report where they demonstrated the pitch change of supramolecular helices created with tripeptide amphiphiles with UV or Visible light from 70 to $40 \mathrm{~nm}$.

Perhaps the most successful commercial products to date have been photochromic or transition lenses. These are lenses made of glass or plastic that contain photochromic molecules which react to ambient UV light to darken, but will return to the colorless form once the stimuli is removed. In this application the utilization of a thermally unstable colored form is key. Initial lenses were based on the switching properties of silver salts. Modern lenses have switched to organic molecules, spironaphthoxazine for example, allowing for a lighter more comfortable plastic. ${ }^{20}$ In fact the first commercial UV-responsive contact lenses are now available. ${ }^{21}$

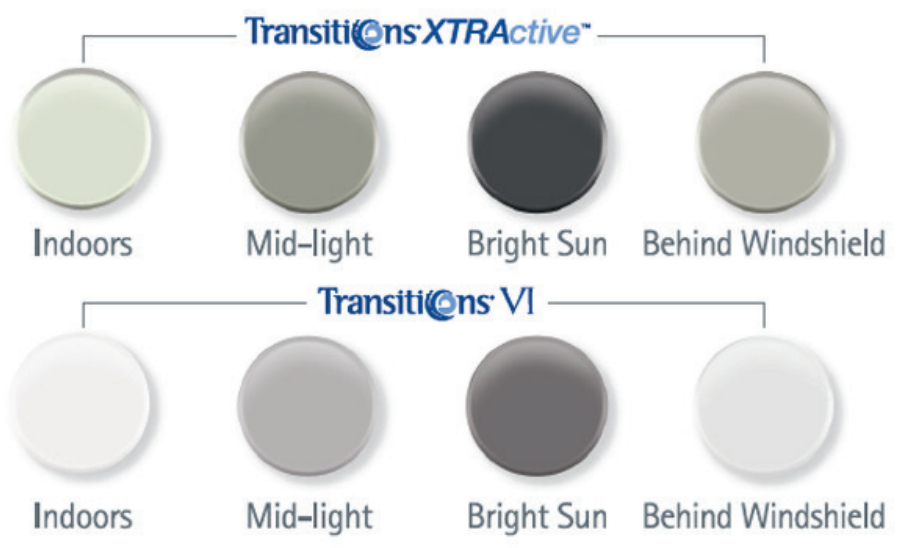

Figure 4: Transition glasses from Transitions Optical, Inc. http://ophthalmiclenses.blogspot.de

Of great importance for this thesis is the switching or modulation of fluorescence with photochromic molecules. This application will be presented in detail in the next section.

Within this thesis I worked exclusively with the family of diheteroarylethenes originally discovered by Masahiro Irie in the late 1980 's. ${ }^{22}$ The principal structural change within diheteroarylethenes is the reversible cyclization analogous to that of a 1,3,5-hexatriene to 1,3cyclohexadiene. ${ }^{23}$ The cyclization reaction occurs through the $\pi-\pi^{*}$ excited state, the conrotary mechanism resulting in a stereospecific product (either S,S or R,R) as can be observed in Scheme $4 .^{24}$ In solution the result is a racemic mixture of the enantiomers, though the use of chiral substituents may favor one over the other. ${ }^{25}$ While stilbenes, though photochromic, are very unstable in the colored form, the benzothiophenes in diheteroarylethenes stabilize the photocyclized structure, making it thermally perdurable. ${ }^{23}$ 
A (Z)-Hexatriene

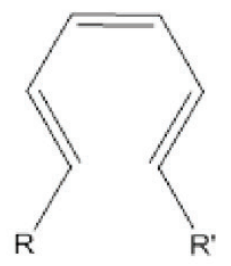

(R,R)-cyclohexa-1,3-diene

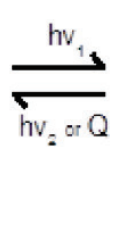

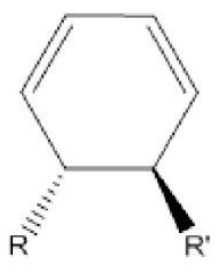

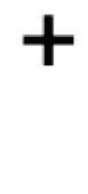

(S,S)-cyclohexa-1,3-diene

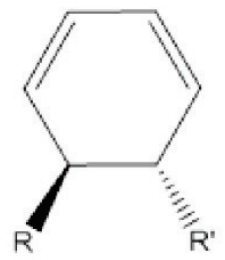

B
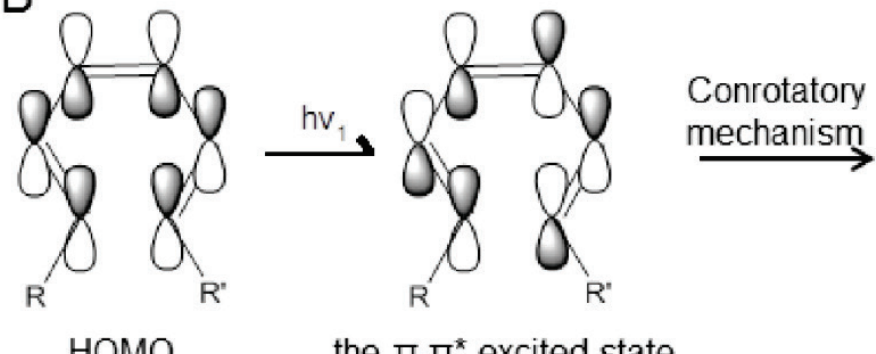

the $\pi-\Pi^{*}$ excited state
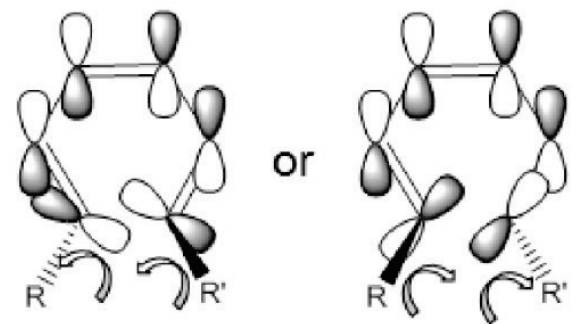

Scheme 4: A) Photocyclization B) Conrotatory mechanism resulting in a specific enantiomer.

The cycled or closed form is colored, due to the extended $\pi$-conjugated system, while the open form is colorless. Chemical modification of the family of diheteroarylethenes is extensive. Studies have focused on creating new bridging moieties, modifying the heteroatom, creating symmetrical/asymmetrical compounds, addition of electron donating/subtracting groups, as well as fluorescence properties. These chemical modifications can have effects on properties such as conversion rates and quantum yields, thermal stability, stereo conformations, fatigue, extinction coefficients, and emission wavelengths. For my experiments diheteroarylethenes presented a few critical advantages, these being: i) a lack of thermal reversion, meaning only irradiation would switch them ii) high fatigue resistance, they are able to go through hundreds of cycles iii) tailoring of optical properties through chemical modification. ${ }^{26}$

The photochromism of diheteroarylethenes, which will henceforth be abbreviated as PCs, has been demonstrated in solution, films, solids, monocrystals and in nanoconstructs. ${ }^{27}$ Due to the extensive aromatic nature of the molecule both the solubility and photoconversion of PCs in water is minimal. Though some recent works have obtained a functional water-soluble ${ }^{28} \mathrm{PC}$ most strategies to overcome this limitation have been through different scaffolds that provide the aqueous solubility as well as a hydrophobic microenvironment for the photoconversion. The work presented within this thesis focuses on PCs within polymer or nanoparticle scaffolds as reversible FRET acceptors. 


\section{Förster Resonance Energy Transfer}

In some ways, life on Earth as we know it is possible due to FRET. Photosynthesis requires the efficient transfer of energy from the photon absorbing antenna complexes to the ATP producing core. This allows the cells to efficiently utilize a much larger range of the spectra than if they were limited to chlorophyll's absorbance. The energy is transferred via FRET, the nontrivial energy transfer from an electronically excited molecule $\mathrm{D}^{*}$ to an acceptor molecule A, through coupling of the two respective oscillating dipoles. The acceptor molecule can then decay to the groundstate through radiative or non-radiative pathways. The rigorous mathematical treatment was first shown by Theodor Förster $^{29}$ in 1948 while in Göttingen.
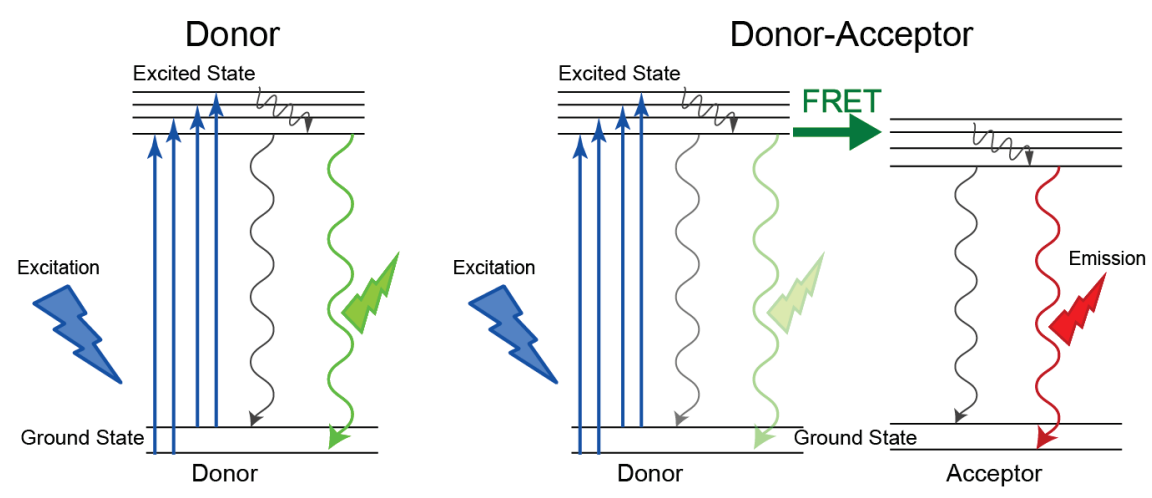

Scheme 5: Jablonski diagram of the donor alone and a donor-acceptor system undergoing FRET.

When the donor (D) is excited by a photon it can revert to the ground state through emission of a photon, in other words it fluoresces. The lifetime of the fluorescence depends on the available decay pathways and FRET adds a non-radiative process to the possible decay pathways. The fluorescence lifetime of the donor is therefore shorter when experiencing FRET than in the absence of an acceptor (A). The efficiency of the energy transfer can be expressed and measured by the following equations.

$$
E=1-\left(\tau_{D A} / \tau_{D}\right)
$$

where $E$ is the FRET efficiency, $\tau_{D A}$ is the donor fluorescence lifetime in presence of energy transfer and, $\tau_{D}$ is the donor fluorescence lifetime without energy transfer. A second way of writing the efficiency, in this case as a function of the donor-acceptor distance, can be expressed as:

$$
E=1 /\left[1+\left(r_{D A} / R_{o}\right)^{6}\right]
$$

where $r_{D A}$ is the donor-acceptor distance, and $R_{o}$ is the Förster distance, which is defined as the donoracceptor distance at which $\mathrm{E}=0.5 . R_{o}$ can be calculated with the following equation

$$
R_{o}^{6}=C_{o} k^{2} J n^{-4} Q_{D}
$$

Where $C_{o}$ is a constant $\left(8.8 \times 10^{-28}\right.$ if $R_{o}$ is expressed in $\left.\mathrm{nm}\right), k^{2}$ an orientation factor of the dipoles, $n$ is the average refractive index of the medium in which the energy transfer occurs, $Q_{D}$ is the 
donor quantum yield in absence of an acceptor, and $J$ is the spectral overlap of the donor emission and the acceptor absorbance. ${ }^{30}$

The orientation factor plays a key role in the energy transfer, but is difficult to know precisely except in the most rigid of environments. The $k^{2}$ value can be between $0-4$, yet is almost exclusively assumed to be $2 / 3$ a value for the situation of freely rotating and random oriented donor-acceptor pairs. ${ }^{30}$ Throughout my work I also assumed a value of $2 / 3$ for $k^{2}$.

Of principal importance when selecting a FRET pair is the resulting $J$ integral.

$$
J=\int_{0}^{\infty} I_{D}(\lambda) \varepsilon_{A}(\lambda) \lambda^{4} \partial \lambda
$$

where $I_{D}(\lambda)$ is the normalized donor fluorescence spectrum and $\varepsilon_{A}(\lambda)$ is the acceptor extinction coefficient. The most intuitive of the factors, it can be envisaged by overlying the emission and absorbance spectra of the donor-acceptor pair. The $J$ integral is the modified parameter in the photochromic FRET discussed below.

Most $R_{o}$ values are in the range of tens of Angstroms, and as can be seen in Equation 2, the efficiency decays with the donor-acceptor distance to the sixth power. Therefore FRET is an extremely sensitive tool for determining distances in the nanometer range. Beyond the natural world FRET is found in varied applications. FRET's acute distance dependence makes it an excellent molecular or calibration ruler. Recently rigid DNA origami has served as a controlled system for systematically varying acceptor-donor distances. ${ }^{31}$ This allows for the study of instrumental or physical factors that must be compensated for.

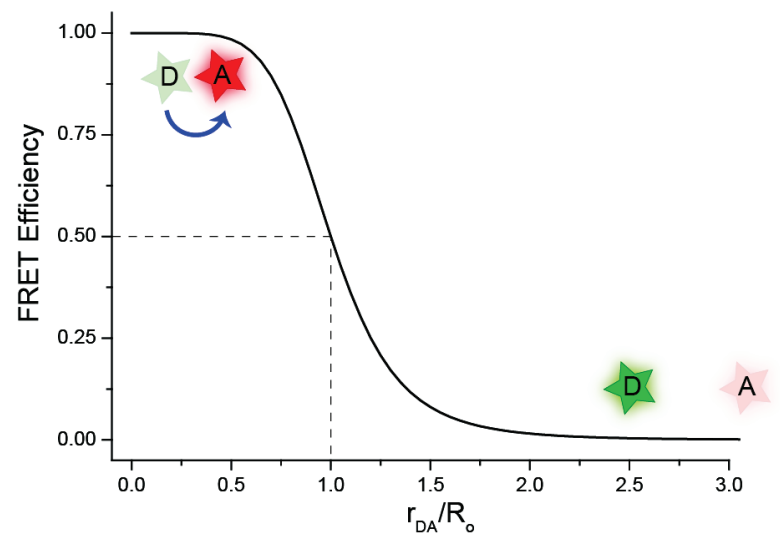

Figure 5: Efficiency of FRET as the donor-acceptance distance varies.

The development of improved fluorescent probes has already been seen in the advances in smFRET (single molecule FRET) measurements. smFRET allows for the interrogation of single proteins, protein-nucleotides, or protein-protein systems so as to resolve information about their folding and interactions. ${ }^{32}$ The simplest example is a 2-state transition which changes the probes on the protein from a FRET state to a non-FRET state. By creating histograms of the dwell times in each 
state the kinetic information about transitions can be determined. The folding mechanism of Chymotrypsin Inhibitor 2, a two-state affair occurring in the millisecond scale, was one of the first proteins studied due to all the previous information known about the system. ${ }^{33}$ When this benchmark was met with smFRET it opened up the field to many other systems such as Cold Shock Protein and some Ribonucleases. ${ }^{32}$ The accessible biological systems have broadened as multicolor smFRET and ALEX (alternating laser excitation) has allowed for the study of more complex systems. ${ }^{34}$

FRET can also be extremely useful in microscopy, both in steady-state and lifetime modes. A comprehensive review on FRET in microscopy by Jares-Erijman and Jovin ${ }^{30}$ is available along with a bevy of reviews covering new developments. Table 1 presents the most common methods for determining FRET.

Table 1: Common FRET imaging methodologies

\begin{tabular}{|c|c|c|}
\hline Name [acronym] & Short description & Reference \\
\hline $\begin{array}{l}\text { Sensitized Emission } \\
{[\mathrm{SE}]}\end{array}$ & $\begin{array}{l}\text { Only the donor is excited, which transfers energy to the acceptor. } \\
\text { Changes in acceptor emission correlate with FRET. }\end{array}$ & 35,36 \\
\hline $\begin{array}{l}\text { Two-channel } \\
\text { Ratiometric FRET } \\
\text { Microscopy }\end{array}$ & $\begin{array}{l}\text { Similar to SE, but the whole gamut of excitation and emissions are } \\
\text { measured. The ratio of donor-acceptor emission is measured in all } \\
\text { conditions. This allows for correction of spectral bleedthrough. }\end{array}$ & $30,36,37$ \\
\hline $\begin{array}{l}\text { Acceptor } \\
\text { Photobleaching FRET } \\
\text { Microscopy [AP] }\end{array}$ & $\begin{array}{l}\text { Donor intensity is measured before and after photobleaching of the } \\
\text { acceptor. Increase in donor emission correlates with FRET. }\end{array}$ & $38-40$ \\
\hline $\begin{array}{l}\text { Fluorescence Lifetime } \\
\text { Imaging [FLIM] }\end{array}$ & $\begin{array}{l}\text { Fluorescence lifetime of the donor and/or acceptor are measured. } \\
\text { Equation [1] showed how lifetime is affected by FRET. FLIM } \\
\text { overcomes concentration limitations and allows for the } \\
\text { determination of both FRET efficiency and the fraction of } \\
\text { interacting partners. }\end{array}$ & $41-43$ \\
\hline $\begin{array}{l}\text { Polarization Anisotropy } \\
\text { Imaging }\end{array}$ & $\begin{array}{l}\text { The anisotropy of the donor and/or acceptor is determined. If FRET } \\
\text { occurs between misaligned pairs, the emission will be polarized } \\
\text { differently than the independent donor or acceptor. }\end{array}$ & 44,45 \\
\hline $\begin{array}{l}\text { MultiColor FRET } \\
\text { Microscopy }\end{array}$ & $\begin{array}{l}\text { Allows for less burdensome determination of multicomponent } \\
\text { interactions. Based on SE emission detection where sequential } \\
\text { FRET can occur (D1 to D2 then to D3) along with direct FRET (D1 } \\
\text { to D3 and D2 to D3). Algorithm based software is then utilized to } \\
\text { unmix the signals and determine corrected efficiencies. }\end{array}$ & $32,34,46,47$ \\
\hline
\end{tabular}




\section{Photochromic Förster Resonance Energy Transfer}

The first article on photochromic FRET (pcFRET) was published by the lab of Dr. Elizabeth Jares-Erijman in 2002. In the paper by Giordano et $a l^{48}$ PC is used as FRET acceptors for lucifer yellow cadaverine. The innovation of pcFRET is that the change in FRET efficiency is not due to a positional change between the acceptor and donor (both $r_{D A}$ and $k^{2}$ are unchanged) but a large alteration in the overlap integral $J$. As stated previously, UV irradiation can modify the conformation of the photochrome, modifying the spectral properties of the acceptor and in turn changing the overlap integral $J$ from zero to a finite value and back again. This can be achieved by modifying the donor fluorescence or the acceptor absorbance.

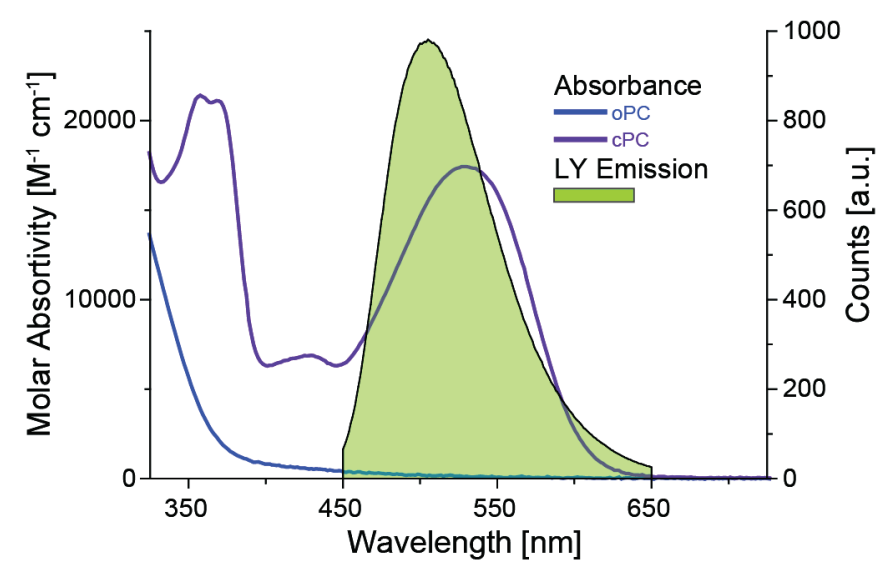

Figure 6: Spectra showing the overlap of the lucifer yellow emission with the open (oPC) or closed (cPC) diheteroarylethene absorbance.

Since its publication a whole new field of photochromic FRET has opened up, the original publication already nearing 200 citations. The implication for photomodulatable probes has been immense, in part because of the advantage of having the photochrome and the donor as two spatially near yet independent entities. This allows for the use of high quantum yield dyes without reducing their brightness.

The Marriot lab is on the forefront of photoswitchable probes for imaging and recently published work with optical lock in detection (OLID) and a pcFRET pair of tetramethylrhodamine (donor) and spironaphthoxazine (acceptor) within living cells. OLID microscopy has been shown to improve contrast while the tetramethylrhodamine is a validated probe for single-molecule resolution, making this a very promising pair. ${ }^{49}$ While the former pcFRET pair was chemically synthesized and then taken up unspecifically by cells, genetically encoded probes can also be utilized. In a different publication they demonstrated the capability to use overexpressed GFP (green fluorescent protein) as a donor for nitrobenzospiropyran that was targetted to the GFP through a SNAP-tag. Using the photoswitching of the acceptor they were able to detect labeling degrees as low as $4 \%{ }^{50}$ 

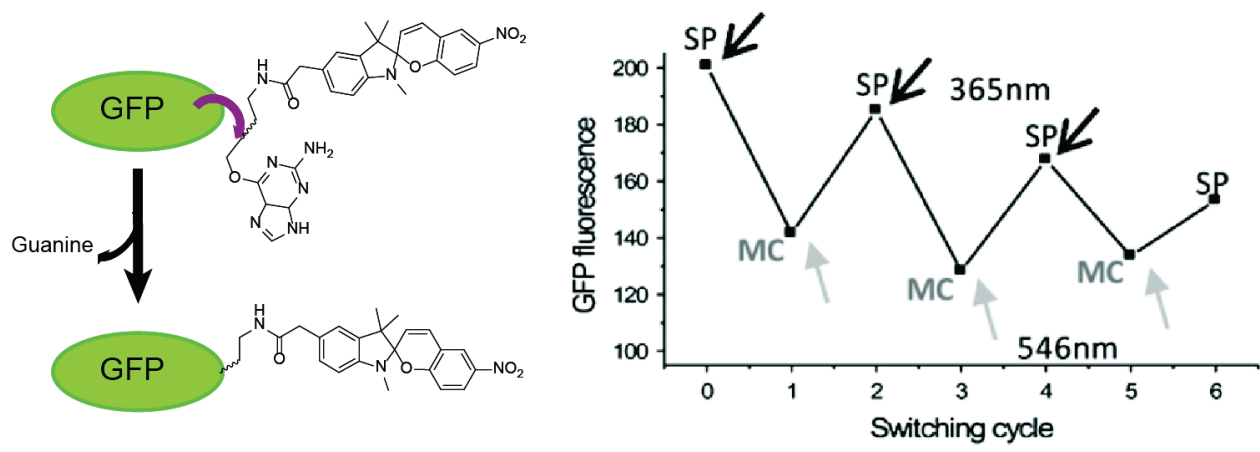

Figure 7: A) Schematic representation of the in vitro and in vivo labeling of a GFP-AGT fusion protein with an optical switch B) The GFP fluorescence change of GFP(NitroBIPS) in response to 1 min irradiation with $365 \mathrm{~nm}$ $\mathrm{UV}^{50}$

pcFRET has been applied very successfully to nanoparticle (NP) probes. As mentioned previously NP scaffolds provide the hydrophobic environment in which photochromic molecules switch more efficiently as well as the aqueous solubility. The reviews of $\operatorname{Tian}^{51}$ and Yildiz ${ }^{52}$ along with their co-authors provide excellent overviews. More recent examples include the use of polymer dots (small NP made of fluorescent polymers, P-dot) doped with PC to quench the intrinsic fluorescence of the P-dot. These P-dots have been observed, along with their photomodulation, at the single dot level as well as being used in cellular microscopy. ${ }^{53,54}$ Amphiphilic polymers were also utilized to create micelles which trap pcFRET dyads composed of boron-dipyrromethene donors and oxazine acceptors. The entraped pcFRET pair could be modulated in water at microsecond switching speeds. ${ }^{55}$ Finally, Wu et $a l^{56}$ demonstrated a NP probe that was not only modulated by light, but the FRET was modulated by temperature as well by placing the acceptor (benzopyrans) on a thermoresponsive PNIPAM polmyer. Higher temperatures cause the polymer, which is bound as a surface ligand to the nanoparticle, to collapse around the surface. This brings the FRET pair nearer increasing the efficiency, but the spectral overlap is small unless the benzopyran is irradiated into the acceptor form. ${ }^{56}$

A

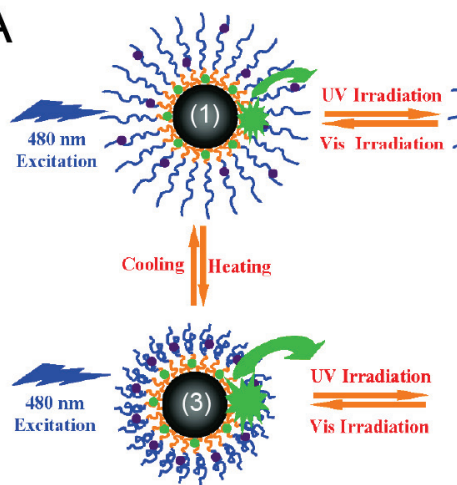

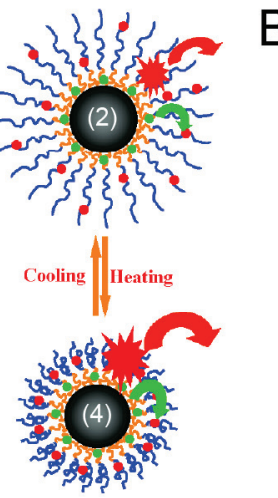

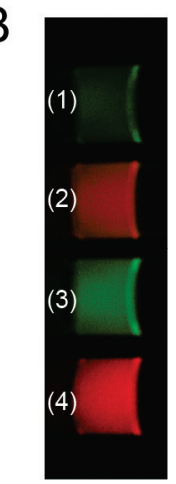

Scheme 6: A) Reversible Modulation of a Multicolor Fluorescent System Fabricated in which the FRET process can be switched on or off via UV or Vis Irradiation and the FRET Efficiency can be tuned via thermo-induced collapse/swelling of responsive polymer B) Photographs Immediately Recorded under a $365 \mathrm{nmUV}$ Lamp at Varying Conditions: (1) $20{ }^{\circ} \mathrm{C}$, upon Vis Irradiation, (2) $20{ }^{\circ} \mathrm{C}$, upon UV Irradiation, (3) $35{ }^{\circ} \mathrm{C}$, upon Vis Irradiation, and (4) $35^{\circ} \mathrm{C}$, upon UV Irradiation. ${ }^{56}$ 
This thesis presents the utilization of diheteroarylethenes as pcFRET acceptors of both organic dyes and inorganic QDs.

\section{Nanoparticles}

Colloidal NPs have been around for millennia; most famously as coloring agents in stained glass as far back as 1000 B.C. ${ }^{57}$ NPs are constructs where the lengths and diameters must be in the one to hundreds of nanometers scale. At this size, interactions due to surface effects or with visible light wavelengths (which can be 2-3 orders of magnitude larger than the NP) can result in phenomenon differing from the bulk properties. NPs can be organic [polymersomes, dendrimers, polymeric nanocapsules], or primarily inorganic, as in the case of metallic $[\mathrm{Au}, \mathrm{Ag}, \mathrm{Pt}]$, magnetic $\left[\mathrm{Fe}_{3} \mathrm{O}_{4}\right]$, silica based $\left[\mathrm{SiO}_{2}\right]$, and QDs. ${ }^{58,59} \mathrm{NPs}$ may also present quantum effects due to their small size. Due to their nano-size the surface area to volume ratio is shifted to much higher ratios than in bulk material. This property frequently provides an advantage in surface-related catalysis, the automobile converter and their platinum NPs serving as the classic example.

QDs present quantum confinement effects; this causes the emission band-gap in the semiconductor to scale with the size of the NP. At this time a broad range of commercial QDs are available varying in size and composition. Due to their broad application, including in this thesis, I will focus on Type I CdSe/ZnS core-shell QDs.

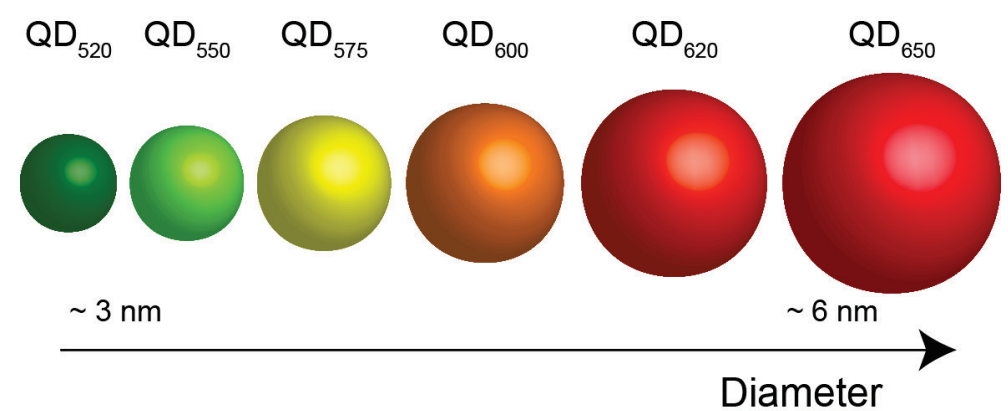

Figure 8: The QD emission wavelength scales with the diameter of the QD core (CdSe in example).

$\mathrm{CdSe} / \mathrm{ZnS}$ QDs are normally prepared in solution, creating colloidal QDs with CdSe cores that can range from 2.5 to $8 \mathrm{~nm}$ in diameter and from blue $(450 \mathrm{~nm})$ to near infrared $(800 \mathrm{~nm})$ in emission. ${ }^{60}$ The outer ZnS passivation shell accounts for the higher quantum yield of core-shell QDs and is usually 1-2 monolayers thick, with final sizes of 4 to $10 \mathrm{~nm}$ in diameter. ${ }^{61}$ The $\mathrm{ZnS}$ has a larger band-gap than the CdSe, making it a type I QD where both charge carriers, the electron and hole, are confined in the core material where the radiative recombination eventually occurs. The alignment of the lattice parameters of the core and outer shell is crucially important. Mismatched crystals will create strain at the interfaces, resulting in defects that defeat the purpose of passivation shells. ${ }^{62}$ The passivation shell not only increases the quantum yield but also avoids surface defects and oxidation/bleaching of the QD. 
A

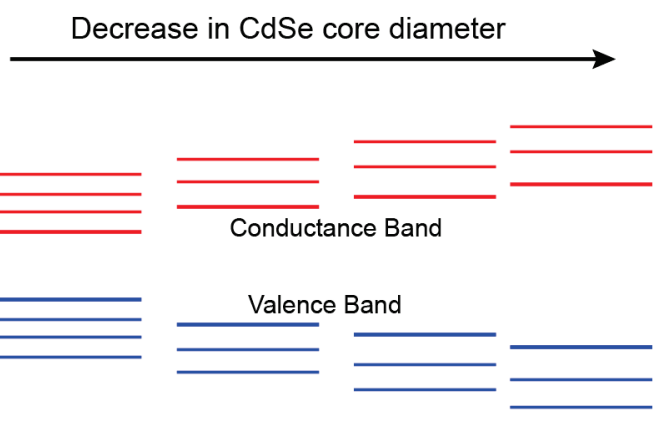

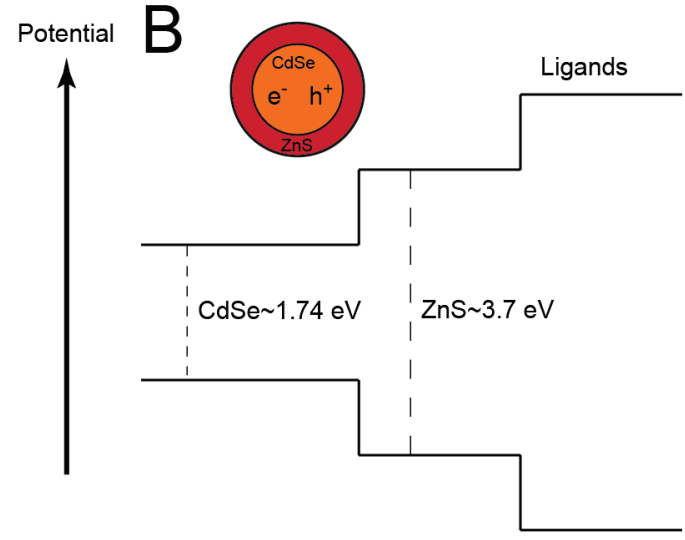

Scheme 7: A) Band-gap scaling with CdSe core size. B) Semiconductor potentials of CdSe/ZnS QDs.

During the formation of the QDs, the surface ligands are present in solution. These molecules regulate the final size of the NP and are responsible for the final stability in solution. ${ }^{63}$ The most common ligands are alkyl amines (dodecylamine, octadecylamine, 1-hexadecylamine), trioctylphosphine (TOP), and trioctyl phosphine oxide (TOPO). These ligands stabilize the QDs in the organic solvent in which the QDs are prepared, but for transfer to aqueous medium, and biological applications, secondary steps are required. The two basic methodologies involve either ligand replacement or ligand capping. Ligand replacement utilizes a second mono or bidentate ligand to displace the original ligands, while this new ligand usually contains a charged head or PEGs that provide the water solubility. This results in smaller QDs, though emission quantum yields are typically diminished. ${ }^{64}$ The capping strategy is based on the utilization of an amphiphilic molecule. The hydrophobic part interacts with the original surface ligands while the hydrophilic section provides the stability. This methodology usually results in larger constructs but the quantum yields are larger and further functionalization, for example targeting, is simpler. ${ }^{64}$ Within my work I utilized a modified amphiphilic polymer to both functionalize the QDs and transfer them to an aqueous medium in one step.

When considering FRET systems that involve QDs as donor and/or acceptors it is important to remember that the QDs can present variations from the classical case along with their own advantages and disadvantages. Excellent reviews on QDs in FRET systems can be found from the Mattoussi and Rogach labs. ${ }^{65-67}$ QDs can be designed with high QYs and long lived excited-state lifetimes making them efficient FRET donors, but the same extended lifetimes make QDs poor FRET acceptors. A second characteristic of QDs are their large size, as compared to traditional organic dyes. This characteristic also provides benefits as well as drawbacks. A larger size allows QD donors to interact with multiple FRET acceptors, but this can lead to complex heterogeneous systems ${ }^{68}$ as will be seen in Chapter 3. The large size of QDs can also be a limit to how close the acceptor can be placed to the actual QD dipole as most $\mathrm{R}_{\mathrm{o}}$ are in the single-digit $\mathrm{nm}$ range and some QDs have diameters as large as $50 \mathrm{~nm}$. The high stoke-shift and broad excitation of QDs make them optimal donors for SE measurements as generally a convenient wavelength that only excites the QD can be found. When the 
$r_{\mathrm{DA}}$ is much larger than the spatial extension of the dipoles, which is the usual case for organic dyes, the classical FRET equation (Equation 2) is valid. QDs do not possess perfect point dipoles, are often times unsymmetrical and surface charges can affect FRET efficiency, but these variations are unpredictable and may have only minor effects. A $k^{2}$ value of $2 / 3$ was selected for FRET analysis, an approximation which is consistent with the scientific consensus when working with a QD-dye D-A pair. ${ }^{68,69}$ Within this thesis QDs were assumed as point dipoles originating from the center of the QD on which the classical FRET equation can be applied.

\section{Scope}

The scope of the thesis entails the preparation and characterization of a water soluble photoswitchable QD that can be used in microscopy. To this end I present in Chapter 1 the synthesis of a series of photochromic diheteroarylethenes. These were prepared with either an aromatic amine or primary amines with different linker lengths. The amine terminated diheteroarylethenes were then conjugated with a poly[isobutylene-alt-maleic anhydride] polymer with numerous alkyl chains to create a photochromic amphiphilic comb polymer. The photochromic properties of the PCs were characterized before and after conjugation to the polymer.

In Chapter 2 a polymer was prepared with PC and Lucifer yellow (LY) that can function as a FRET donor to the PC. The PC-LY pcFRET pair and the resulting fluorescence photomodulation is characterized in 3 different constructs; i) as individual polymer strands in organic solvent; ii) as large multi-strand polymersomes in buffer solution; iii) coated on a QD template suspended in buffer solution.

Chapter 3 presents the preparation and characterization of photoswitchable quantum dots (psQDs) as well as a psQD which contains a secondary internal standard so as to create ratiometric imaging capabilities. These are spectroscopically characterized with absorbance and steady-state and time-correlated fluorescence. A photophysical model is postulated that explains the photoconversion and photomodulation data.

Chapter 4 focuses on the single-particle investigation of the psQDs utilizing laser scanning confocal and TIRF (total internal reflection fluorescence) microscopy setups. Information is gleaned about the effects of the photochromes and their state on the photophysical properties of the QDs.

Chapter 5 contains a series of proof-of-concept experiments where psQDs were modified with targeting capabilities and then used in cellular microscopy to demonstrate the possible applications. 


\section{References}

1. Hell, S. W.; Wichmann, J. Breaking the Diffraction Resolution Limit by Stimulated-Emission Stimulated-Emission-Depletion Fluorescence Microscopy. Opt. Lett. 1994, 19, 780-782.

2. Dertinger, T.; Colyer, R.; Iyer, G.; Weiss, S.; Enderlein, J. Fast, Background-Free, 3d Super-Resolution Optical Fluctuation Imaging (SOFI). Proc. Natl. Acad. Sci. U. S. A. 2009, 106, 22287-22292.

3. Rust, M. J.; Bates, M.; Zhuang, X. W. Sub-Diffraction-Limit Imaging by Stochastic Optical Reconstruction Microscopy (STORM). Nat. Methods 2006, 3, 793-795.

4. Beyond the Diffraction Limit. Nat. Photon. 2009, 3, 361-361.

5. $\quad$ Baker, M. Microscopy: Bright Light, Better Labels. Nature 2011, 478, 137-142.

6. Jares-Erijman, E.; Giordano, L.; Spagnuolo, C.; Lidke, K.; Jovin, T. M. Imaging Quantum Dots Switched on and Off by Photochromic Fluorescence Resonance Energy Transfer (pcFRET). Mol. Cryst. Liquid Cryst. 2005, 430, 257-265.

7. Diaz, S. A.; Menendez, G. O.; Etchehon, M. H.; Giordano, L.; Jovin, T. M.; Jares-Erijman, E. A. Photoswitchable Water-Soluble Quantum Dots: pcFRET Based on Amphiphilic Photochromic Polymer Coating. ACS Nano 2011, 5, 2795-2805.

8. Duerr, H.; Bouas-Laurent, H., Photochromism Molecules and Systems. In Studies in Organic Chemistry, 1990; Vol. 40, p 1068.

9. Hirshberg, Y.; Fischer, E. Low-Temperature Photochromism and Its Relation to Thermochromism. $J$. Chem. Soc. (Resumed) 1953, 629.

10. Ishii, N.; Kato, T.; Abe, J. A Real-Time Dynamic Holographic Material Using a Fast Photochromic Molecule. Sci. Rep. 2012, 2.

11. Bruder, F. K.; Hagen, R.; Rolle, T.; Weiser, M. S.; Facke, T. From the Surface to Volume: Concepts for the Next Generation of Optical-Holographic Data-Storage Materials. Angew. Chem.-Int. Edit. 2011, 50, 4552-4573.

12. Kawata, S.; Kawata, Y. Three-Dimensional Optical Data Storage Using Photochromic Materials. Chem. Rev. 2000, 100, 1777-1788.

13. Parthenopoulos, D. A.; Rentzepis, P. M. Three-Dimensional Optical Storage Memory. Science 1989, $245,843-845$.

14. Uchida, K.; Saito, M.; Murakami, A.; Kobayashi, T.; Nakamura, S.; Irie, M. Three Bits Eight States Photochromic Recording and Nondestructive Readout by Using Ir Light. Chem.-Eur. J. 2005, 11, 534542.

15. Pu, S. Polarization Multiplexing Holographic Optical Recording of a New Photochromic Diarylethene. Opt. Eng. 2008, 47, 030502.

16. Han, M.; Michel, R.; He, B.; Chen, Y.-S.; Stalke, D.; John, M.; Clever, G. H. Light-Triggered Guest Uptake and Release by a Photochromic Coordination Cage. Angew. Chem.-Int. Edit. 2013, 52, 13191323.

17. Yamaguchi, H.; Kobayashi, Y.; Kobayashi, R.; Takashima, Y.; Hashidzume, A.; Harada, A. Photoswitchable Gel Assembly Based on Molecular Recognition. Nat. Comm. 2012, 3, 603.

18. Coleman, A. C.; Beierle, J. M.; Stuart, M. C. A.; Macia, B.; Caroli, G.; Mika, J. T.; van Dijken, D. J.; Chen, J. W.; Browne, W. R.; Feringa, B. L. Light-Induced Disassembly of Self-Assembled VesicleCapped Nanotubes Observed in Real Time. Nat. Nanotechnol. 2011, 6, 547-552. 
19. Li, L.-s.; Jiang, H.; Messmore, B. W.; Bull, S. R.; Stupp, S. I. A Torsional Strain Mechanism to Tune Pitch in Supramolecular Helices. Angew. Chem.-Int. Edit. 2007, 119, 5977-5980.

20. Pardo, R.; Zayat, M.; Levy, D. Photochromic Organic-Inorganic Hybrid Materials. Chem. Soc. Rev. 2011, 40, 672 .

21. Nabais, C. R. J. O. D.; Heron, B. M.; de Sousa, H. C.; Gil, M. H.; Sobral, A. J. F. N. Synthesis and Characterization of Co-Polymers Based on Methyl Methacrylate and 2-Hexyl Acrylate Containing Naphthopyrans for a Light-Sensitive Contact Lens. J. Biomater. Sci., Polym. Ed. 2011, 22, 139-152.

22. Irie, M.; Mohri, M. Thermally Irreversible Photochromic Systems. Reversible Photocyclization of Diarylethene Derivatives. J. Org. Chem. 1988, 53, 803-808.

23. Nakamura, S.; Irie, M. Thermally Irreversible Photochromic Systems - a Theoretical-Study. J. Org. Chem. 1988, 53, 6136-6138.

24. Woodward, R.; Hoffmann, R., The Conservation of Orbital Symmetry. Verlag Chemie: New York, 1970 .

25. Pace, T. C. S.; Müller, V.; Li, S.; Lincoln, P.; Andréasson, J. Enantioselective Cyclization of Photochromic Dithienylethenes Bound to DNA. Angew. Chem.-Int. Edit. 2013, 52, 4393-4396.

26. Irie, M. Diarylethenes for Memories and Switches. Chem. Rev. 2000, 100, 1685-1716.

27. Irie, M. Photochromism of Diarylethene Single Molecules and Single Crystals. Photochem. Photobiol. Sci. 2010, 9, 1535-1542.

28. Polyakova, S. M.; Belov, V. N.; Bossi, M. L.; Hell, S. W. Synthesis of Photochromic Compounds for Aqueous Solutions and Focusable Light. Eur. J. Org. Chem. 2011, 3301-3312.

29. Förster, T. Zwischenmolekulare Energiewanderung Und Fluoreszenz. Ann. Phys. 1948, 437, 55-75.

30. Jares-Erijman, E. A.; Jovin, T. M. FRET Imaging. Nat. Biotechnol. 2003, 21, 1387-1395.

31. Stein, I. H.; Schuller, V.; Bohm, P.; Tinnefeld, P.; Liedl, T. Single-Molecule FRET Ruler Based on Rigid DNA Origami Blocks. Chemphyschem 2011, 12, 689-695.

32. Michalet, X.; Weiss, S.; Jäger, M. Single-Molecule Fluorescence Studies of Protein Folding and Conformational Dynamics. Chem. Rev. 2006, 106, 1785-1813.

33. Deniz, A. A.; Laurence, T. A.; Beligere, G. S.; Dahan, M.; Martin, A. B.; Chemla, D. S.; Dawson, P. E.; Schultz, P. G.; Weiss, S. Single-Molecule Protein Folding: Diffusion Fluorescence Resonance Energy Transfer Studies of the Denaturation of Chymotrypsin Inhibitor 2. Proc. Natl. Acad. Sci. U. S. A. 2000, 97, 5179-5184.

34. Gambin, Y.; Deniz, A. A. Multicolor Single-Molecule FRET to Explore Protein Folding and Binding. Mol. BioSyst. 2010, 6, 1540.

35. Sun, Y. S.; Wallrabe, H.; Seo, S. A.; Periasamy, A. FRET Microscopy in 2010: The Legacy of Theodor Forster on the 100th Anniversary of His Birth. Chemphyschem 2011, 12, 462-474.

36. Ishikawa-Ankerhold, H. C.; Ankerhold, R.; Drummen, G. P. C. Advanced Fluorescence Microscopy Techniques-FRAP, FLIP, FLAP, FRET and FLIM. Molecules 2012, 17, 4047-4132.

37. Pietraszewska-Bogiel, A.; Gadella, T. W. J. FRET Microscopy: From Principle to Routine Technology in Cell Biology. J. Microsc.-Oxf. 2011, 241, 111-118.

38. Gadella, T. W. J.; Jovin, T. M. Oligomerization of Epidermal Growth-Factor Receptors on A431 Cells Studied by Time-Resolved Fluorescence Imaging Microscopy - a Stereochemical Model for Tyrosine Kinase Receptor Activation. J. Cell Biol. 1995, 129, 1543-1558. 
39. Van Munster, E. B.; Kremers, G. J.; Adjobo-Hermans, M. J. W.; Gadella, T. W. J. Fluorescence Resonance Energy Transfer (FRET) Measurement by Gradual Acceptor Photobleaching. J. Microsc.Oxf. 2005, 218, 253-262.

40. Wang, L.; Chen, T.; Qu, J.; Wei, X. Photobleaching-Based Quantitative Analysis of Fluorescence Resonance Energy Transfer inside Single Living Cell. J.Fluoresc. 2010, 20, 27-35.

41. Chen, Y. C.; Clegg, R. M. Fluorescence Lifetime-Resolved Imaging. Photosynth. Res. 2009, 102, 143155.

42. Padilla-Parra, S.; Audugé, N.; Coppey-Moisan, M.; Tramier, M. Quantitative FRET Analysis by Fast Acquisition Time Domain FlLIMat High Spatial Resolution in Living Cells. Biophys. J. 2008, 95, 29762988.

43. Oida, T.; Sako, Y.; Kusumi, A. Fluorescence Lifetime Imaging Microscopy (Flimscopy). Methodology Development and Application to Studies of Endosome Fusion in Single Cells. Biophys. J. 1993, 64, 676-685.

44. Lidke, D. S.; Nagy, P.; Barisas, B. G.; Heintzmann, R.; Post, J. N.; Lidke, K. A.; Clayton, A. H. A.; Arndt-Jovin, D. J.; Jovin, T. M. Imaging Molecular Interactions in Cells by Dynamic and Static Fluorescence Anisotropy (rFLIM and emFRET). Biochem. Soc. T. 2003, 31, 1020-1027.

45. Chan, F. T. S.; Kaminski, C. F.; Schierle, G. S. K. Homofret Fluorescence Anisotropy Imaging as a Tool to Study Molecular Self-Assembly in Live Cells. Chemphyschem 2011, 12, 500-509.

46. Preus, S.; Wilhelmsson, L. M. Advances in Quantitative Fret-Based Methods for Studying Nucleic Acids. ChemBioChem 2012, 13, 1990-2001.

47. Lam, J. C. F.; Li, Y. Influence of Cleavage Site on Global Folding of an Rna-Cleaving DNAzyme. ChemBioChem 2010, 11, 1710-1719.

48. Giordano, L.; Jovin, T. M.; Irie, M.; Jares-Erijman, E. A. Diheteroarylethenes as Thermally Stable Photoswitchable Acceptors in Photochromic Fluorescence Resonance Energy Transfer (pcFRET). J Am Chem Soc 2002, 124, 7481-7489.

49. Yan, Y.; Marriott, M. E.; Petchprayoon, C.; Marriott, G. Optical Switch Probes and Optical Lock-in Detection (OLID) Imaging Microscopy: High-Contrast Fluorescence Imaging within Living Systems. Biochem. J. 2011, 433, 411-422.

50. Mao, S.; Benninger, R. K. P.; Yan, Y.; Petchprayoon, C.; Jackson, D.; Easley, C. J.; Piston, D. W.; Marriott, G. Optical Lock-in Detection of FRET Using Synthetic and Genetically Encoded Optical Switches. Biophys. J. 2008, 94, 4515-4524.

51. Tian, Z.; Wu, W.; Li, A. D. Photoswitchable Fluorescent Nanoparticles: Preparation, Properties and Applications. Chemphyschem 2009, 10, 2577-2591.

52. Yildiz, I.; Deniz, E.; Raymo, F. M. Fluorescence Modulation with Photochromic Switches in Nanostructured Constructs. Chem Soc Rev 2009, 38, 1859-1867.

53. Osakada, Y.; Hanson, L.; Cui, B. Diarylethene Doped Biocompatible Polymer Dots for Fluorescence Switching. Chem. Comm. 2012, 48, 3285-3287.

54. Davis, C. M.; Childress, E. S.; Harbron, E. J. Ensemble and Single-Particle Fluorescence Photomodulation in Diarylethene-Doped Conjugated Polymer Nanoparticles. J. Phys. Chem. C 2011, 115, 19065-19073.

55. Cusido, J.; Battal, M.; Deniz, E.; Yildiz, I.; Sortino, S.; Raymo, F. M. Fast Fluorescence Switching within Hydrophilic Supramolecular Assemblies. Chem.-Eur. J. 2012, 18, 10399-10407. 
56. Wu, T.; Zou, G.; Hu, J.; Liu, S. Fabrication of Photoswitchable and Thermotunable Multicolor Fluorescent Hybrid Silica Nanoparticles Coated with Dye-Labeled Poly(N-Isopropylacrylamide) Brushes. Chem. Mat. 2009, 21, 3788-3798.

57. Sciau, P., Nanoparticles in Ancient Materials: The Metallic Lustre Decorations of Medieval Ceramics. InTech: Rijeka, Croatia, 2012.

58. Mulder, W. J.; Strijkers, G. J.; van Tilborg, G. A.; Cormode, D. P.; Fayad, Z. A.; Nicolay, K. Nanoparticulate Assemblies of Amphiphiles and Diagnostically Active Materials for Multimodality Imaging. Acc Chem Res 2009, 42, 904-914.

59. Hahn, M. A.; Singh, A. K.; Sharma, P.; Brown, S. C.; Moudgil, B. M. Nanoparticles as Contrast Agents for in-Vivo Bioimaging: Current Status and Future Perspectives. Anal Bioanal Chem 2011, 399, 3-27.

60. Norris, D. J.; Efros, A. L.; Rosen, M.; Bawendi, M. G. Size Dependence of Exciton Fine Structure in CdSe Quantum Dots. Phys.Rev. B 1996, 53, 16347-16354.

61. Dabbousi, B. O.; Rodriguez-Viejo, J.; Mikulec, F. V.; Heine, J. R.; Mattoussi, H.; Ober, R.; Jensen, K. F.; Bawendi, M. G. (Cdse)Zns Core-Shell Quantum Dots: Synthesis and Characterization of a Size Series of Highly Luminescent Nanocrystallites. J. Phys. Chem. B 1997, 101, 9463-9475.

62. Talapin, D. V.; Mekis, I.; Götzinger, S.; Kornowski, A.; Benson, O.; Weller, H. CdSe/CdS/ZnS and CdSe/ZnSe/ZnS Core-Shell-Shell Nanocrystals. J. Phys. Chem. B 2004, 108, 18826-18831.

63. Evans, C. M.; Love, A. M.; Weiss, E. A. Surfactant-Controlled Polymerization of Semiconductor Clusters to Quantum Dots through Competing Step-Growth and Living Chain-Growth Mechanisms. $J$ Am Chem Soc 2012, 134, 17298-17305.

64. Sperling, R. A.; Parak, W. J. Surface Modification, Functionalization and Bioconjugation of Colloidal Inorganic Nanoparticles. Philos Transact A Math Phys Eng Sci 2010, 368, 1333-1383.

65. Medintz, I. L.; Clapp, A. R.; Mattoussi, H.; Goldman, E. R.; Fisher, B.; Mauro, J. M. Self-Assembled Nanoscale Biosensors Based on Quantum Dot FRET Donors. Nat. Mater. 2003, 2, 630-638.

66. Medintz, I. L.; Uyeda, H. T.; Goldman, E. R.; Mattoussi, H. Quantum Dot Bioconjugates for Imaging, Labelling and Sensing. Nat. Mater. 2005, 4, 435-446.

67. Rogach, A. L.; Klar, T. A.; Lupton, J. M.; Meijerink, A.; Feldmann, J. Energy Transfer with Semiconductor Nanocrystals. J. Mater. Chem. 2009, 19, 1208-1221.

68. Medintz, I. L.; Mattoussi, H. Quantum Dot-Based Resonance Energy Transfer and Its Growing Application in Biology. Phys. Chem. Chem. Phys. 2009, 11, 17-45.

69. Chung, I. H.; Shimizu, K. T.; Bawendi, M. G. Room Temperature Measurements of the 3d Orientation of Single CdSe Quantum Dots Using Polarization Microscopy. Proc. Natl. Acad. Sci. U. S. A. 2003, $100,405-408$. 


\section{CHAPTER 1: SYNTHESIS AND CHARACTERIZATION OF DIHETEROARYLETHENES AND POLYMERS}

\section{Introduction}

Photochromic diheteroarylethenes (PC) have various chemically modifiable sub-structures. Though any number of the preexisting variants could well have served my purpose I chose 3,3'(3,3,4,4,5,5-hexafluorocyclopent-1-ene-1， 2-diyl)bis(2-methylbenzo[b]thiophene) as the base structure. The base PC presents efficient photoconversion, fatigue resistance, an absorbance peak in the closed form in the $540 \mathrm{~nm}$ range, and negligible fluorescence emission. In Scheme 1 the selected PC molecule is presented and the molecule is divided in 3 sub-sections that will be further explored below. As shorthand, open PC molecules will be abbreviated as oPC and the closed or photocyclized form as $\mathrm{cPC}$.
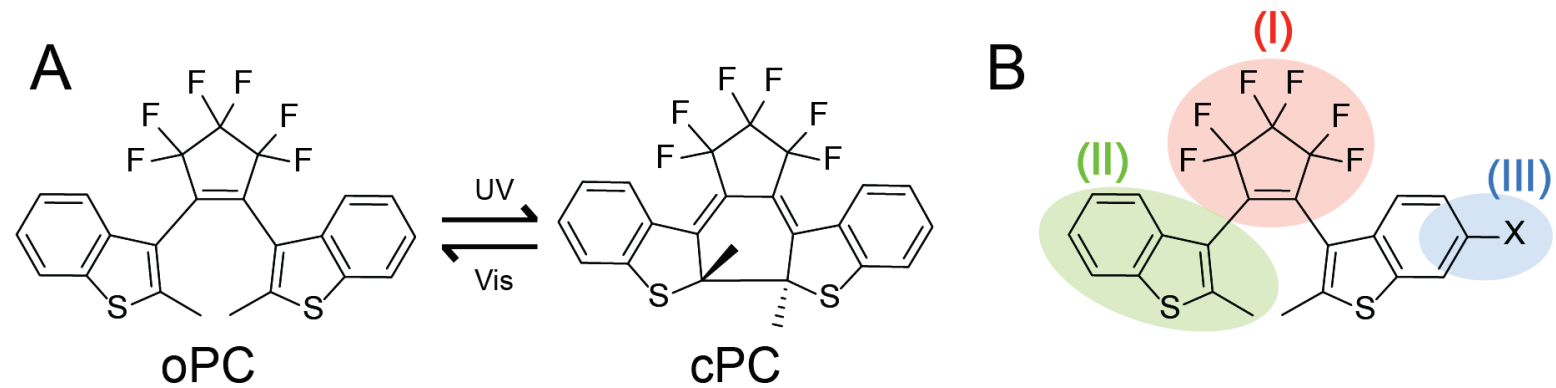

Scheme 1: A) Photoconversion of oPC and cPC B) Sub-sections of PC.

The first sub-section (I) is the bridging unit. The literature shows a variety of bridging units available to chemists. These include but are not limited to cyclobutenes, maleic anhydrides, heterocycles, cyclopentenes, and hexafluorcyclopentenes, ${ }^{1,2}$ the latter being chosen for use in my thesis. Bridging units are normally cyclic molecules to avoid cis-trans transitions of the central double bond during photocycles. ${ }^{3}$ Hexafluorcyclopentenes provide excellent fatigue resistance by avoiding oxidation pathways. They also present excellent photochromic properties normally favoring the kinetics of ring closure, while the ring opening is less favored. This tendency can be enhanced by choosing 6 carbon ringed bridges or depressed by selecting 4 carbon rings. ${ }^{4}$

The second section (II) is the core of the PC. The core clearly plays a large role in the photochromic and absorbance properties. I utilized a 2-methylbenzo[b]thiophene as a symmetric core. The thiophene core is crucial for the thermal stability of the molecule. The thermal interconversion between the open and closed forms depends on the activation energy barriers. The open and closed conformations of thiophene cores are closer in their base energy levels, eliminating any large driving force, and causing both the open and closed form to be thermally stable. ${ }^{5}$ The aromatic ring extends the $\pi$ - conjugation, creating a molecule with an increased extinction coefficient, which is important for its capability as a FRET acceptor. 
It was mentioned that the cyclized bridges avoid cis-trans transitions, but there is another stereo confirmation that plays a large role in the photocyclization. In the open form PC molecules can present both a parallel (mirror symmetry) and anti-parallel ( $\mathrm{C}_{2}$ symmetry) conformation. The ratio of conformers is important because only the antiparallel conformation is capable of photocycling. ${ }^{6}$ For this study the 2-methylbenzo[b]thiophene core was chosen due to the straight-forward and economic synthesis available for the PC, along with the aforementioned photophysical advantages.

Parallel

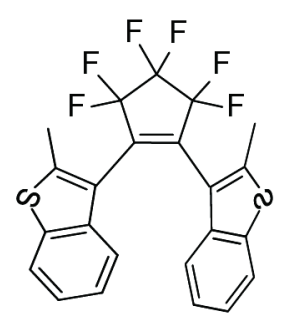

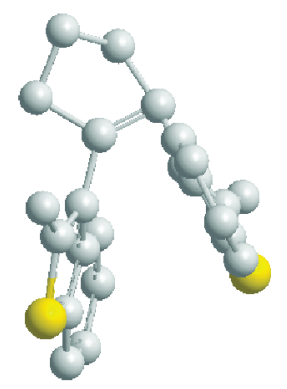
Anti-parallel

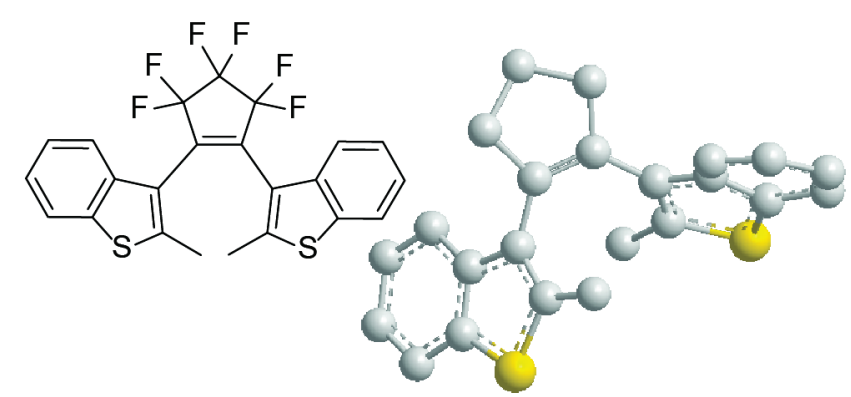

Scheme 2: Parallel and anti-parallel conformation of oPC

The third highlighted section (III) is where the PC functionalization was realized. The PC was modified at the 6-C position to create reactive groups capable of coupling to the polymers and proteins as will be seen in Sections 1.2 and 1.3.

The PC is a principal building block for the preparation of the functionalized amphiphilic polymer. The other components are dodecylamine, and poly[isobutylene-alt-maleic anhydride] (PMA), an alternating copolymer of succinic anhydride rings separated by butylene residues. The PMA backbone is strategically favorable since it is economical ( 150€/kilo), commercially available, and the anhydride groups react with primary amines to form covalent amide bonds. A quick literature search shows a number of photochromic polymers, ${ }^{7}$ yet to the best of my knowledge, my work was the first report of a photochromic PMA-based amphiphilic polymer. ${ }^{8}$ The final polymer is a comb-like amphiphile that allows for the transfer of the QDs to an aqueous medium.

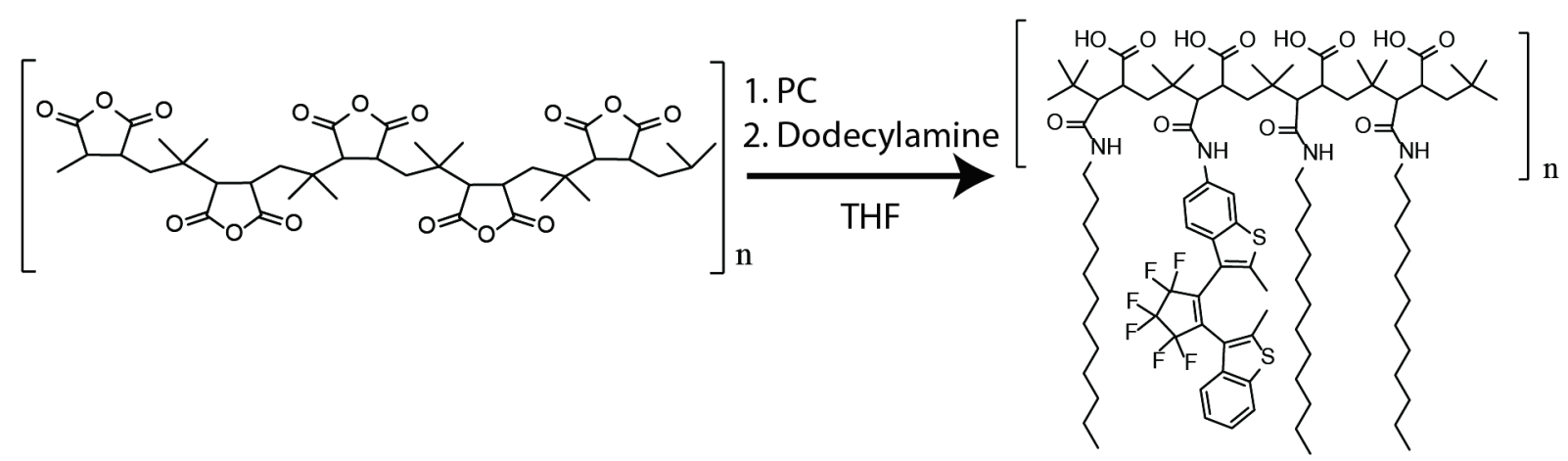

Scheme 3: PMA and preparation of photoswitchable comb-like amphiphile.

The first publications on PMA based polymers for NP coating was put forth by the lab of Wolfgang Parak. ${ }^{9,} 10$ They demonstrated that it was a versatile strategy for transferring any organic NP 
to aqueous medium. Most importantly the polymer could be modified prior to or after nanoparticle coating with very simple chemistry. Due to the previously mentioned limitations of PCs in buffers, where they are insoluble and lack photoconversion, the post-coating strategy was not feasible with the PC. By directly modifying the polymer, one can place hydrophobic moieties within the apolar microenvironment that is formed upon self-assembly. The technique has been utilized with various functionalizations and entire protocols are now available. ${ }^{11}$

\subsection{Synthesis of Base Diheteroarylethene (PC)}

The starting molecule was 2-methylbenzo[b]thiophene with the initial step for the PC synthesis being the halogenation of this molecule in position 3 . The resulting organoiodine compound was chosen due to its increased reactivity in subsequent steps. In general, iodine is a weak electrophile, only capable of iodating phenols or amines. The addition of oxidizing agents and strong acids, in this case periodic acid and sulfuric acid respectively, catalyzes the reaction. Though the actual electrophile is not confirmed, it is postulated that the most likely agent is $\mathrm{I}^{+}$(see Scheme 4). ${ }^{12}$ The reaction lasts approximately $2 \mathrm{~h}$ at $\mathrm{RT}$ and is then quenched with $\mathrm{NaHSO}_{3}$ and $\mathrm{NaOH}$ and extracted with $\mathrm{CH}_{2} \mathrm{Cl}_{2}$. The product, 3-iodo-2-methylbenzo[b]thiophene, is recrystallized in $\mathrm{MeOH} / \mathrm{H}_{2} \mathrm{O}$ to obtain a white crystal. $\left(\mathrm{R}_{\mathrm{f}}=0.62\right.$ in $100 \%$ n-heptane; yield, $\left.90 \%\right)$.

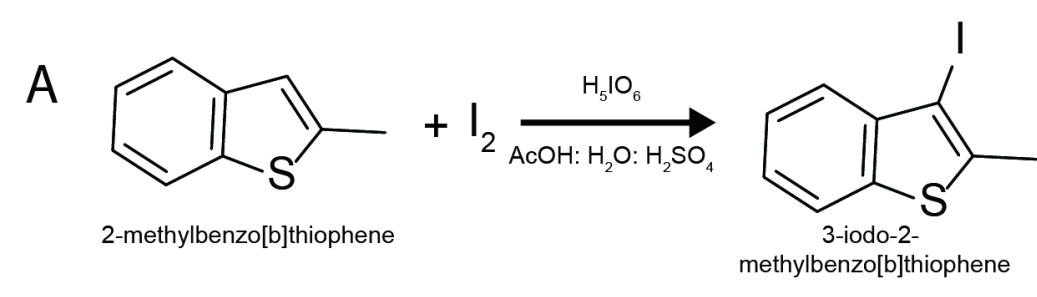

B

$$
\mathrm{H}_{5} \mathrm{IO}_{6}+3 \mathrm{I}_{2}+7 \mathrm{H}^{+} \rightarrow 7 \mathrm{I}^{+}+6 \mathrm{H}_{2} \mathrm{O}
$$

Scheme 4: A) Synthesis of 3-iodo-2-methylbenzo[b]thiophene B) Postulated formation of I ${ }^{+}$electrophile.

This product is reacted with the bridging unit, octafluorocyclopentene, to synthesize the PC. Addition of $n$-BuLi to the iodated molecule creates a carbanion by removal of the iodine. The double bond of the octafluorocyclopentene then undergoes a 2-step nucleophilic addition of the carbanion with elimination of LiF. When the addition occurs for a second time the PC is synthesized. The fluorinated alkene is very reactive since the electronegative $F$ group activates the alkene to a nucleophile attack. This reaction requires a precise control of the temperature and anhydrous conditions. If the temperature is not kept at $-78{ }^{\circ} \mathrm{C}$ the carbanion can migrate or attack other groups, and the carbanion will also be destroyed by any traces of water. 


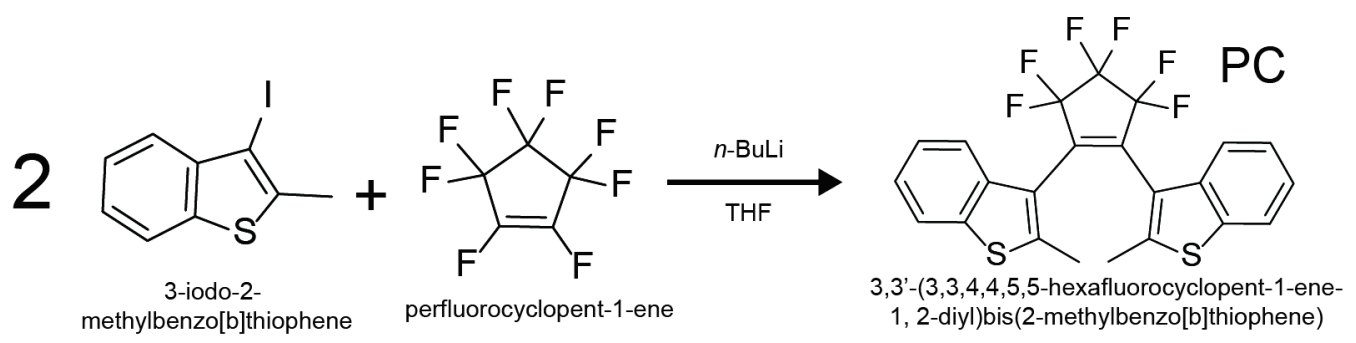

Scheme 5: Synthesis of PC

The product 3,3'-(3,3,4,4,5,5-hexafluorocyclopent-1-ene-1, 2-diyl)bis(2methylbenzo[b]thiophene) is obtained after one hour. The reaction is quenched $(1 \mathrm{~N} \mathrm{HCl})$ and the end product is extracted with $\mathrm{CH}_{2} \mathrm{Cl}_{2}$ to obtain a white-yellow solid. The reaction is monitored by TLC, and the product turns pink under UV light. $\left(\mathrm{R}_{\mathrm{f}}=0.40\right.$ in $100 \% \mathrm{n}$-heptane; yield, $\left.60 \%\right)$.

If the eq. of bridging unit to core are not 1:2 the side product 2-methyl-3-(perfluorocyclopent1-enyl)benzo[b]thiophene (referred to as Half) is obtained. This is the principal side-product of PC synthesis and can be obtained in a pure form during the column purification of the PC. Half can be used as an intermediary of asymmetric PC synthesis as well as a reagent to obtain the symmetric PC. ${ }^{13}$

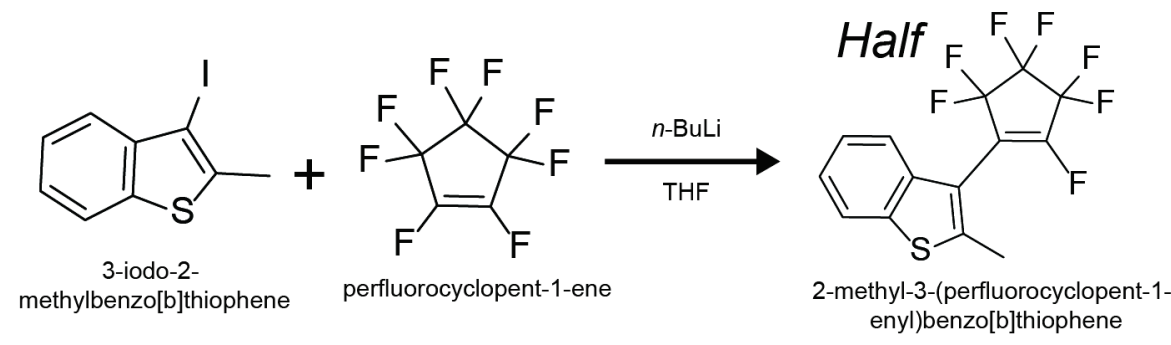

Scheme 6: Synthesis of Half.

The strategy for polymer conjugation involves the synthesis of a series of amine modified PCs which are presented in detail in Section 1.3. A first step requires the asymmetric nitration of position C-6 to obtain the intermediary $\mathrm{PCmNO}_{2}$ [3-(3,3,4,4,5,5-Hexafluoro-2-(2-methylbenzo[b]thiophen- 3yl)cyclopent-1-en-1-yl)-2-methyl-6-nitrobenzo[b]thiophene]. The reaction is an electrophilic aromatic substitution of a hydrogen by a nitro group. The mechanism requires the formation of a nitronium ion $\left(\mathrm{NO}_{2}^{+}\right)$through the mixed-acid pathway. The mixed-acid pathway requires an acidic dehydrating agent as a catalyst in conjunction with fuming nitric acid. To avoid over-nitration acetic acid and acetic anhydride were utilized instead of the more common sulfuric acid. If the reaction is not properly regulated, meaning time $(12 \mathrm{~h})$, concentrations, and temperature $\left(5^{\circ} \mathrm{C} \rightarrow \mathrm{RT}\right)$, the end product is the symmetric C-6 / C-6' $\mathrm{PCdiNO}_{2}$ [3,3'-(perfluorocyclopent-1-ene-1,2-diyl)bis(2-methyl-6nitrobenzo[b]thiophene)].

The end product, $\mathrm{PCmNO}_{2}$, obtained after purification is a light purple solid and reveals as a purple stain on TLC under UV light. $\left(\mathrm{R}_{\mathrm{f}}=0.40\right.$ 97:3 cyclohexane: ethyl acetate; yield, $\left.40 \%\right)$. $\mathrm{PCdiNO}_{2}$ has a similar appearance $\left(\mathrm{R}_{\mathrm{f}}=0.2097: 3\right.$ cyclohexane: ethyl acetate $)$ 


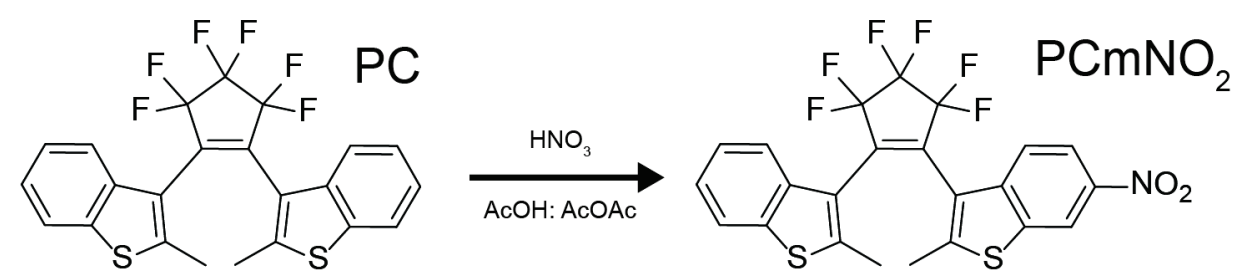

Scheme 7: Synthesis of $\mathrm{PCmNO}_{2}$.

\subsection{Synthesis of PCs for Polymer Conjugation}

\subsubsection{Synthesis of $\mathrm{PCmNH}_{2}$ and of $\mathrm{PCmonomer}$}

The modification of the PMA backbone requires the nucleophilic attack on the succinic anhydride to form a covalent bond. Consequently, within my work primary amines, both aromatic and aliphatic, were selected as the nucleophile of choice. A primary amine will react with succinic anhydride to form an amide bond and a free carboxyl group. The first step was the preparation of a photochromic molecule with an aromatic amine in the C-6 position, 3-(3,3,4,4,5,5-hexafluoro-2-(2methylbenzo[b]thiophen-3-yl)cyclopent-1-enyl)-2-methylbenzo[b]thiophen-6-amine, which will be known as $\mathrm{PCmNH}_{2}$. $\mathrm{PCmNH}_{2}$ can react directly with the PMA, or can be further modified to allow for extended linkers (see Section 1.3).

The preparation of $\mathrm{PCmNH}_{2}$ is based on the reduction of the nitro group from $\mathrm{PCmNO}_{2}$. I utilized a weak reducing agent, $\mathrm{NaBH}_{4}$, since it is economic, environmentally friendly, and allows for gentle reaction conditions. Though $\mathrm{NaBH}_{4}$ alone cannot, for the most part, reduce aromatic nitro groups, in combination with transition metals the reducing power is greatly increased. ${ }^{14}$ The use of $\mathrm{NiCl}_{2} \bullet 6 \mathrm{H}_{2} \mathrm{O}$ as a catalyst generated a quick and high yield reaction.

A solution of $\mathrm{PCmNO}_{2}$ was prepared in methanol with $\mathrm{NiCl}_{2} \cdot 6 \mathrm{H}_{2} \mathrm{O}$ as a catalyst. The solution was placed in an ice bath, and then $\mathrm{NaBH}_{4}$ was slowly added, leading to the immediate formation of a black precipitate. The solution reached completion in $\sim 10 \mathrm{~min}$, was quenched with 0.04 $\mathrm{N} \mathrm{HCl}$, filtered, and then evaporated. The reaction could be followed by TLC due to the change in $\mathrm{R}_{\mathrm{f}}$, but also the product reveals bright pink under UV light, as compared to the purple of the reagent. The residue was extracted with dichloromethane and the organic phase was washed, dried, and evaporated. After purification through a silica column the end product, $\mathrm{PCmNH}_{2}$ was obtained as a pink oil $\left(\mathrm{R}_{\mathrm{f}}=\right.$ $0.25,70: 30$ cyclohexane: ethyl acetate; yield, $75 \%$ ). $\mathrm{PCmNH}_{2}$ is not very stable; it was either used the same day it was prepared, or stored in an argon atmosphere at $-20^{\circ} \mathrm{C}$, and even then its shelf life is $\sim 2$ months. In protic solvents UV irradiation quickly causes the $\mathrm{PCmNH}_{2}$ to degrade, observed as a stark blue shift in the absorbance. 


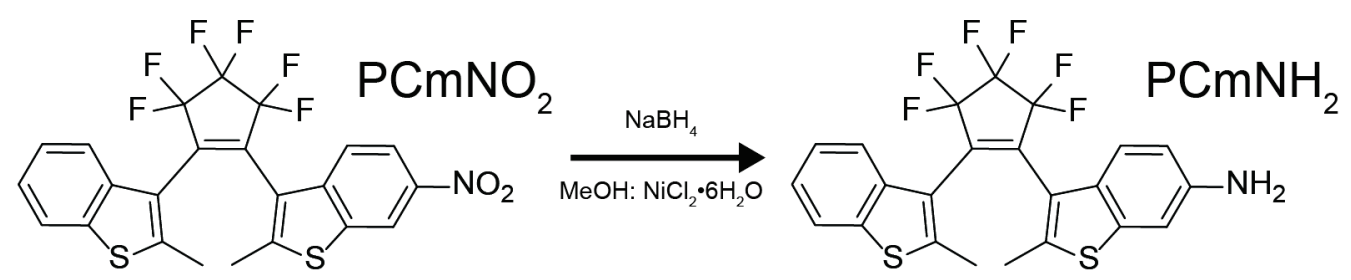

Scheme 8: Synthesis of $\mathrm{PCmNH}_{2}$.

$\mathrm{PCmNH}_{2}$ was the first photochrome I synthesized that would react with the succinic anhydrides of the PMA. As stated the primary amines react to form covalent amide bonds. The properties of an aromatic amine and an aromatic amide are quite different; in fact one is an electron donating group while the amide is an electron withdrawing group. To adequately characterize a molecule that most closely resembled the photochromic group bound to the polymer I prepared a photochromic monomer. PCmonomer is 4-((3-(3,3,4,4,5,5-hexafluoro-2-(2-methylbenzo[b]thiophene3-yl)cyclopent-1-en-1-yl]-2-methylbenzo[b]thiophene-6-yl)amino)-4-oxobutanoic acid and can be observed in Scheme 8.

The reaction consists of the nucleophilic attack of $\mathrm{PCmNH}_{2}$ on a succinic anhydride molecule. The succinic anhydride is prepared in anhydrous THF then mixed with $\mathrm{PCmNH}_{2}$. The solution was heated to $55{ }^{\circ} \mathrm{C}$ and allowed to react for 3 hours. After quenching the reaction and purifying the product, a grey precipitate is obtained which reveals as a pink stain under UV light $\left(\mathrm{R}_{\mathrm{f}}=0.10,5: 3\right.$ cyclohexane: ethyl acetate; yield, 78\%)

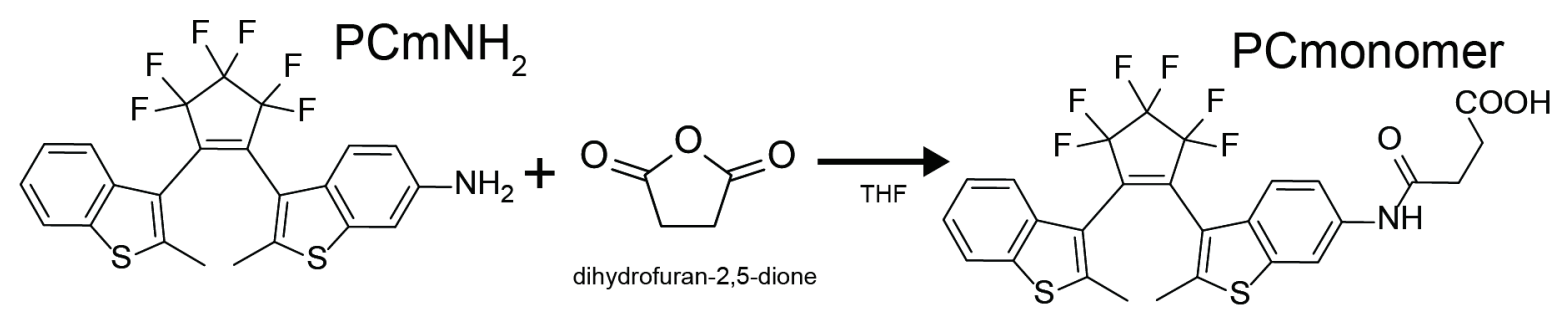

Scheme 8: Synthesis of PCmonomer.

\subsubsection{Characterization of $\mathrm{PCmNH}_{2}$ and of $\mathrm{PCmonomer}$}

The principal interest in the PC molecules is their change in spectra upon irradiation. Samples were irradiated using an Hg arc lamp (SUV-DC, Lumatec) and appropriate filters. For UV irradiation a $340 \pm 10 \mathrm{~nm}$ bandpass filter was utilized, and since only the cPC has an absorbance peak $>500 \mathrm{~nm}$, a $545 \pm 10 \mathrm{~nm}$ bandpass filter was used for the photoreversal. All samples utilized the same set of filters for photocyclization and photoreversal. Samples were placed in Hellma $10 \times 2 \mathrm{~mm}$ microcuvettes, filled such that the entire sample was exposed to light, thereby eliminating mixing effects. 

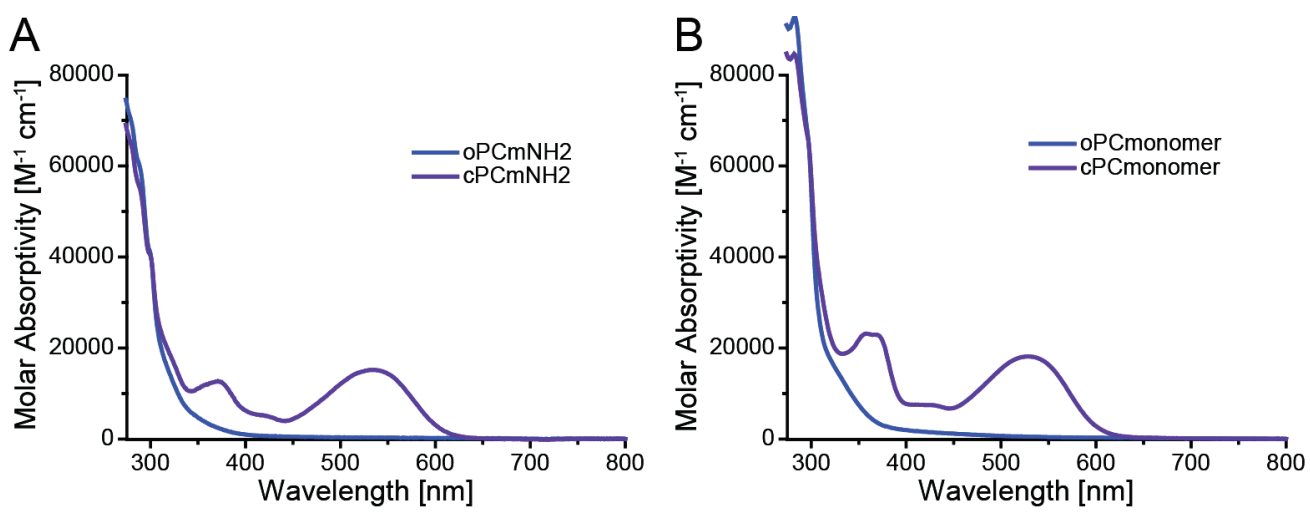

Figure 1: Absorbance spectra of A) $\mathrm{PCmNH}_{2} \mathrm{~B}$ ) $\mathrm{PCmonomer}$

Considerable differences can be observed in the absorbance spectra. When C-6 is changed from the amine to the amide group the peak at $\sim 375 \mathrm{~nm}$ of the closed form has a higher extinction coefficient (see Table 2) and the broad peak at $\sim 550$ is shifted $4 \mathrm{~nm}$ to the blue.

In PC molecules only the antiparallel (ap) form of the open isomer can photocyclize. The interchange from parallel (p) to ap is slower than the excited state lifetime of the molecule, meaning that the quantum efficiency of conversion will always be limited by this ratio. Using NMR spectroscopy the relation of $\mathrm{p}$ to ap confirmation can be easily determined. The protons on the methyl groups of the $\mathrm{C}-2$ position sense different environments depending on the conformation. ${ }^{15}$ Integrating these peaks allows for the determination of the p:ap ratio. Work done previously in the lab of Dr. Jares-Erijman confirmed that the higher field methyl groups are from the parallel conformation. ${ }^{13}$

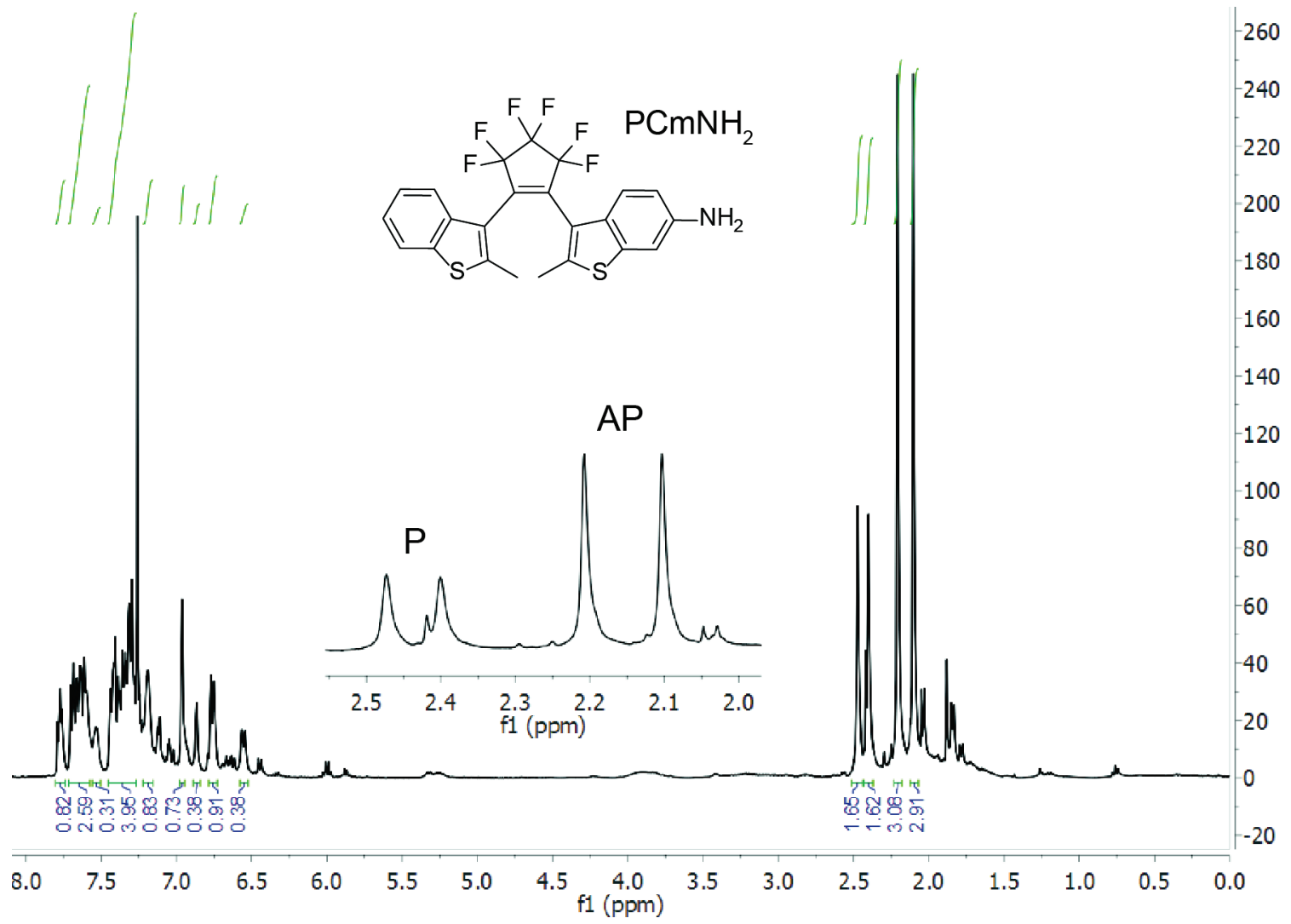

Figure 2: ${ }^{1} \mathrm{H}$ NMR spectra of $\mathrm{PCmNH}_{2}$ in $\mathrm{CDCl}_{3}$. 
The ${ }^{1} \mathrm{H}$ NMR spectra of $\mathrm{PCmNH}_{2}$ is shown in Figure 2.. The inset highlights the methyl peaks where: $\mathrm{CDCl}_{3}, 2.10 \mathrm{ppm}$ (s, 2.91H, CH3 ap), $2.21 \mathrm{ppm}$ (s, 3.08H, CH3 ap), $2.40 \mathrm{ppm}$ (s, 1.62H, CH3 $\mathrm{p}$ ), and $2.47 \mathrm{ppm}(\mathrm{s}, 1.65 \mathrm{H}, \mathrm{CH} 3 \mathrm{p})$. The p:ap ratio was therefore 35:65. The exact value will depend on the solvent of choice, along with temperature and viscosity. In a similar manner all PC can be measured and their a:ap ratio determined. PCmonomer has the same ratio (35:65 in $\mathrm{CHCl}_{3}$ at $\left.298 \mathrm{~K}\right)$ while all other PC molecules based on the amide linker at C-6 present similar values, up to $\sim 40: 60$.

NMR spectroscopy also plays a vital role in determining the conversion factor for PC molecules. Due to the fact that cPC also absorbs UV light, in which case photoreversal may occur, the final concentration of $\mathrm{OPC}$ and $\mathrm{CPC}$ is at equilibrium, regardless of how long one irradiates with $\mathrm{UV}$ light. This final equilibrium point is known as the photostationary state (PS state) and the degree of conversion is $\alpha_{\mathrm{PS}}=\mathrm{cPC} /(\mathrm{cPC}+\mathrm{oPC})$. This equilibrium depends on the wavelength of the photoconversion light and the local environment. The ${ }^{1} \mathrm{H}$ NMR spectra of the open to the closed form were quite different and could be used to determine the degree of photoconversion, $\alpha_{\mathrm{PS}}$, in the photostationary state. ${ }^{16}$

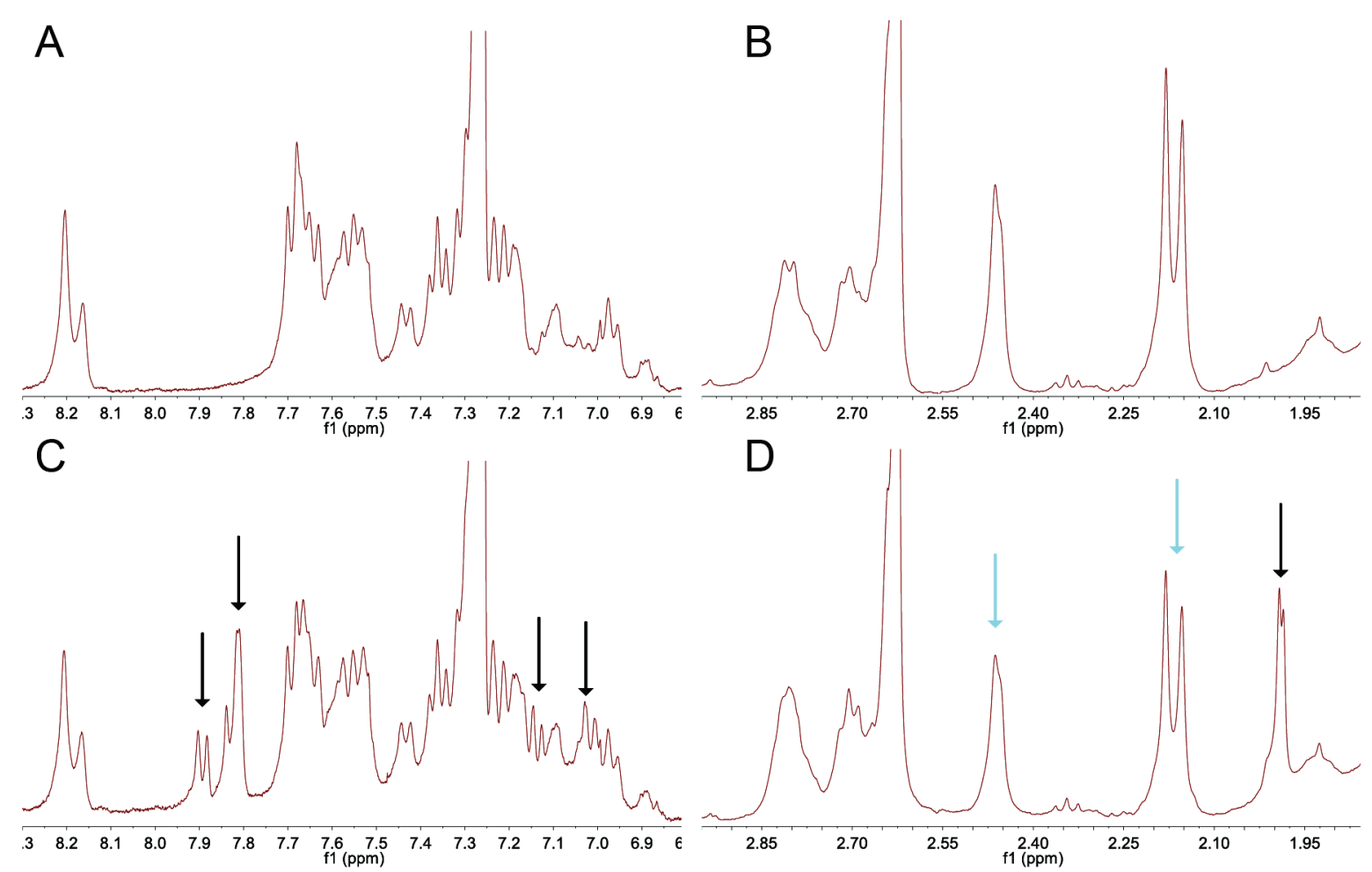

Figure 3: ${ }^{1} \mathrm{H}$ NMR $\mathrm{CDCl}_{3}$ for $\mathrm{PCmonomer}$ in the open $(\mathrm{A}, \mathrm{B})$ and photostationary $(\mathrm{C}, \mathrm{D})$ states. New peaks were observed and are marked with black arrows, while peaks with diminished areas are marked with blue arrows. The areas of the peaks were used to calculate the conversion extent of the PCmonomer. ${ }^{8}$

For quantification of $\alpha_{\mathrm{PS}}$ PCmonomer was utilized since it better represents the PC conjugated to the polymer. In Figure 3 there are two useful regions for quantifying the extent of photoconversion; the integration values are given in Table 1. The methyl peaks showed decreases of 21 and 23\%, corresponding to $\mathrm{CH}_{3}$ ap (2.14 and $2.18 \mathrm{ppm}$ ) and $\mathrm{CH}_{3}$ p (2.46 ppm) hydrogens respectively. The increase of the $1.99 \mathrm{ppm}$ peak corresponds to $22 \%$ of the areas of the three peaks. In the aromatic 
region all peaks are integrated. The new peaks corresponding to cPCmonomer represent $20 \%$ of the area. The overlap from the $\mathrm{CHCl}_{3}$ peak makes the aromatic quantification less precise. In the end I decided that a value of $\alpha_{\mathrm{PS}}=22 \%$ was the best estimation. ${ }^{8}$

Table 1: Integrated values for ${ }^{1} \mathrm{H}$ NMR signals of PCmonomer

\begin{tabular}{llllllll}
\hline \multirow{2}{*}{ State } & \multicolumn{6}{l}{ Peaks (chemical shift, ppm) } \\
& 1.99 & 2.16 & 2.46 & 7.03 & 7.13 & 7.82 & 7.89 \\
\hline Open & 1431 & 12656 & 7152 & - & - & - & - \\
Photostationary & 6309 & 9955 & 5476 & 1610 & 888 & 2145 & 877 \\
\hline
\end{tabular}

Knowing the conversion factor allows the determination of the extinction coefficients. Solutions of the $\mathrm{PC}$ of interest were prepared in $\mathrm{CHCl}_{3}$. Using the law of Lambert-Beer, and the $22 \%$ conversion rate previously calculated, the extinction coefficients were calculated. The wavelengths of interest were $340 \mathrm{~nm}$ ( $\lambda$ used for photocyclization), $400 \mathrm{~nm}$ ( $\lambda$ used for QD excitation), and $545 \mathrm{~nm}$ (maximum of cPC absorbance peak).

Table 2: Extinction coefficients $\left[\mathrm{M}^{-1} \mathrm{~cm}^{-1}\right]$

\begin{tabular}{lllll}
\hline Wavelength & ${ }_{\text {oPCmNH}}$ & $\mathrm{cPCmNH}_{2}$ & oPCmonomer & cPCmonomer \\
\hline $340 \mathrm{~nm}$ & 2075 & 6015 & 2000 & 9100 \\
$400 \mathrm{~nm}$ & 380 & 7700 & 175 & 5730 \\
$545 \mathrm{~nm}$ & 11 & 14800 & 17 & 17100 \\
\hline
\end{tabular}

Assuming that the extinction coefficients do not radically change in different solvents the isosbestic point of the oPC and cPC forms can be utilized to calculate the $\alpha_{\mathrm{PS}}$. The isosbestic point is normally found near $300 \mathrm{~nm}$ as can be seen in Figure 1.

$$
\alpha_{p s}=-\left(\frac{\varepsilon_{o}^{545}}{\varepsilon_{c}^{545}-\varepsilon_{o}^{545}}\right)+\left(\frac{\varepsilon^{i s o s}}{\varepsilon_{c}^{545}-\varepsilon_{o}^{545}}\right)\left(\frac{A_{P S}^{545}}{A^{i s o s}}\right)
$$

The conversion factors varied slightly according to the polarity of the solvent. In order of decreasing polarity; $\mathrm{MeOH}(0.185 \%)$; THF $(0.21 \%)$; $\mathrm{CHCl}_{3}(0.22 \%)$; n-heptane $(0.225 \%)$. Slight differences were observed for different PC moieties, but as the differences were within the experimental error, the entire set of utilized PCs could be represented by these values. 


\subsection{PC with Extended Functionalizations}

\subsubsection{PC with Primary Amine Terminated Aliphatic Linkers}

A series of PCs with different length linkers, all terminating in primary amines, were prepared. This was done for two principal reasons: the first being that primary alkyl amines are more reactive to anhydrides, therefore increasing the yield of conjugation to the polymer. ${ }^{17}$ The second reason is that FRET is very dependent on the donor-acceptor distance. The inclusion of linkers would allow for the polymer conjugated PC to be placed closer to the QD donor. The linkers were commercial molecules which presented a carboxyl group on one end and a protected amine on the other with an aliphatic carbon chain varying in length as the linker.

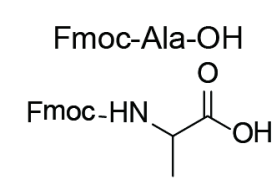

Boc-Ahx-OH<smiles>O=C(O)CCCCCCNC(=O)OCc1ccccc1</smiles>

Fmoc-Aoc-OH

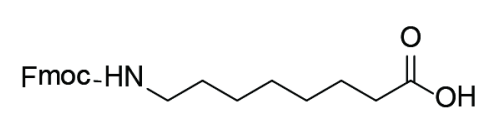

Fmoc-Ado-OH

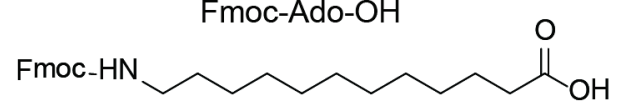

Scheme 9: Commercial linkers used in synthesis, presenting a carboxyl group on one end and a protected amine on the other.

The $\mathrm{PCmNH}_{2}$ was used as the base from which the PCs with linkers were prepared. The aromatic amine of $\mathrm{PCmNH}_{2}$ is not reactive enough to conjugate with simpler methodologies such as EDC [1-Ethyl-3-(3-dimethylaminopropyl)carbodiimide] or NHS-esters. I therefore used acyl chlorides to activate the carboxyl group, allowing a nucleophilic acyl substitution. The acyl chlorides were prepared using thionyl chloride and commercial linkers.
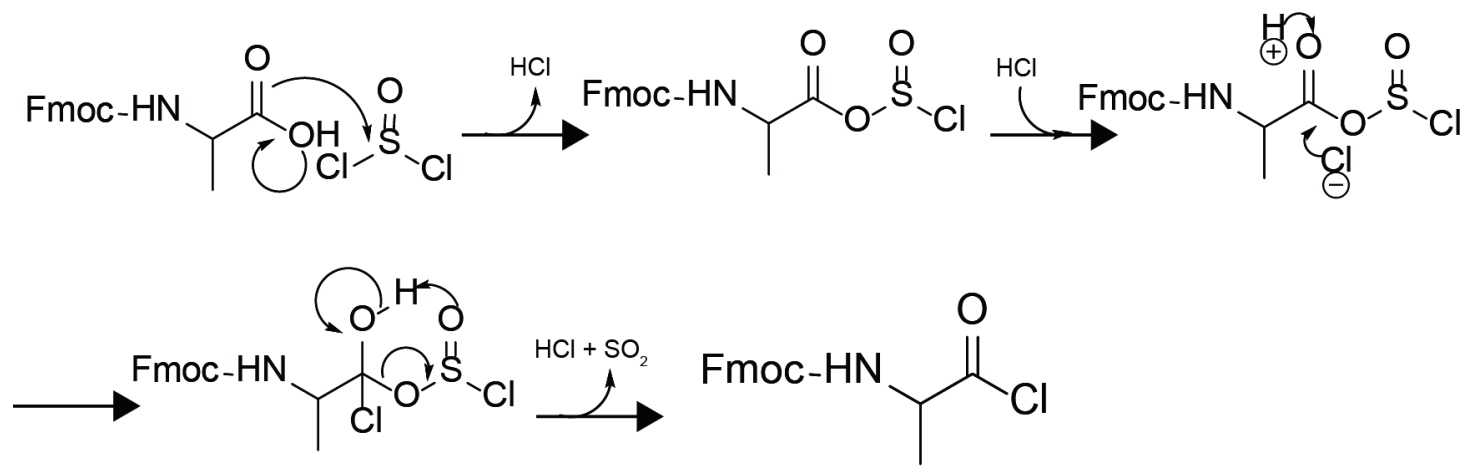

Scheme 10: Mechanism of acyl chloride formation.

The reactive nature of acyl chlorides made it preferable that they be used as soon as possible. Generally the $\mathrm{PCmNH}_{2}$ and acyl chloride were prepared and reacted in the same day. 


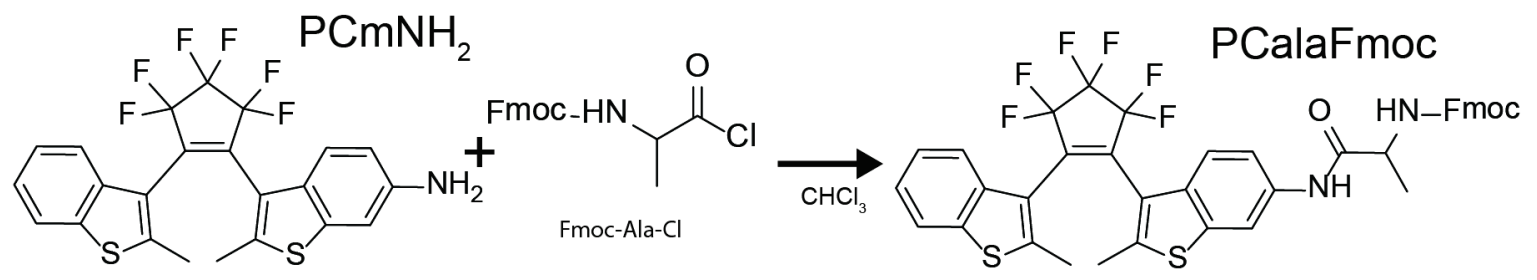

Scheme 11: Synthesis of PCalaFmoc.

The reaction was completed after an hour at RT and was quenched with $\mathrm{NaOH}$ and extracted with $\mathrm{CH}_{2} \mathrm{Cl}_{2}$. The products were purified using a silica column. The following nomenclature was adopted: PC-name of linker-END GROUP. As an example, the molecule in Scheme 11 would be PCalaFmoc. If the protective group was removed it would be $\mathrm{PCalaNH}_{2}$. Table 3 presents the series of prepared PC linkers with protective groups.

Table 3: Description of prepared photochromes.

\begin{tabular}{lclllc}
\hline Molecule & Linker length & Appearance & Yield & $\mathrm{R}_{\mathrm{f}}$ (Mobile phase $)$ & UV light $^{\mathrm{a}}$ \\
\hline PCalaFmoc & $2 \mathrm{C}$ & Red foam & $84 \%$ & $0.30(7: 3$ Cyclohexane: AcOEt $)$ & Pink \\
PCahxBoc & $6 \mathrm{C}$ & Pink oil & $55 \%$ & $0.55(5: 5$ Cyclohexane: AcOEt $)$ & Pink \\
PCaocFmoc & $8 \mathrm{C}$ & Pink wax & $73 \%$ & $0.40(6: 4$ Cyclohexane: AcOEt $)$ & Pink \\
PCadoFmoc & $12 \mathrm{C}$ & Golden oil & $45 \%$ & $0.65(6: 4$ Cyclohexane: AcOEt $)$ & Pink \\
\hline
\end{tabular}

[a] Observed appearance of stain on TLC, revealed with UV irradiation.

The protective groups were removed to obtain the primary amine which was able to react with the PMA. The Boc group was removed using concentrated $\mathrm{HCl}$ in ethyl acetate at $\mathrm{RT}$ after an initial attempt to remove the Boc with pyridine and heating was unsuccessful. The Fmoc groups were removed using piperidine at $50{ }^{\circ} \mathrm{C}$. The reactions were followed by TLC and when the primary amine product was obtained the $\mathrm{R}_{\mathrm{f}}=0$ and the product turned pink under UV light. All products (see Scheme 12) were purified by silica column. The yields for the last step, meaning the liberation of the protective groups, was $\sim 60 \%$. All products had a pink/yellow oil appearance after purification.
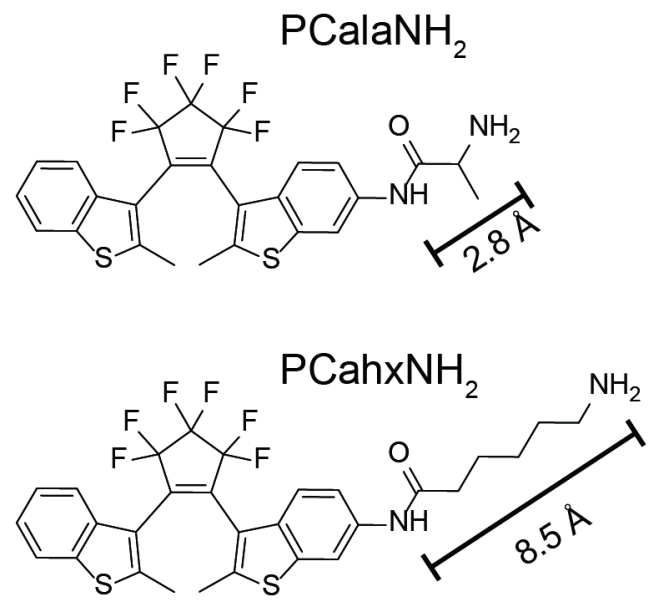
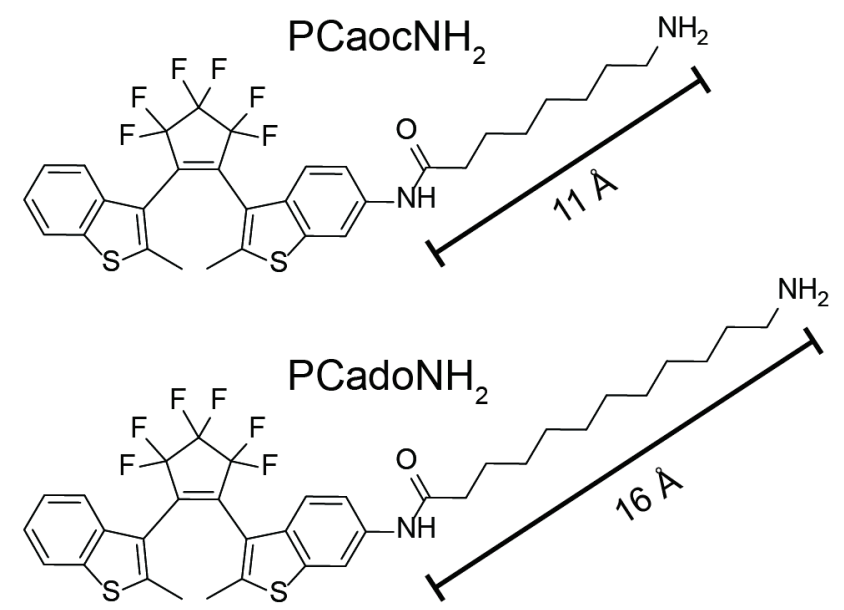

Scheme 12: Photochromic molecules with primary amine terminated aliphatic linkers. Length of the linkers at maximum extension. 
The spectral characterization was realized exactly as in Section 1.2. Structural similarities to PCmonomer suggested that the photophysical properties would be comparable. The values calculated for the $\mathrm{PClinkNH}_{2}$ photochromes were within the expected range and were well represented by the values in Table 2 .

\subsubsection{Photoactivatable Crosslinking PC}

A final PC molecule was synthesized within a collaborative framework of the study of FABP (Fatty Acid Binding Proteins). The contribution consisted in the synthesis of a photochromic molecule required for the testing of the binding pocket of FABP, which can bind to fatty acids, monoglycerides, sterols, and some artificial drugs. ${ }^{18}$ Of particular interest was the development of a photoactivatable crosslinking photochrome with a diazirine, N-(3-(3,3,4,4,5,5-hexafluoro-2-(2methylbenzo[b]thiophen-3-yl)cyclopent-1-enyl)-2-methylbenzo[b]thiophen-6-yl)-2-(3-(3-methyl-3Hdiazirin-3-yl)propanamido)propanamide, was synthesized. The diazirine is activated with UV light creating a radical species that forms a covalent bond with the protein. If the photochrome is inside the beta barrel hydrophobic binding pocket the photoconversion should be efficient and therefore act as a reversible acceptor for a cascade yellow donor.

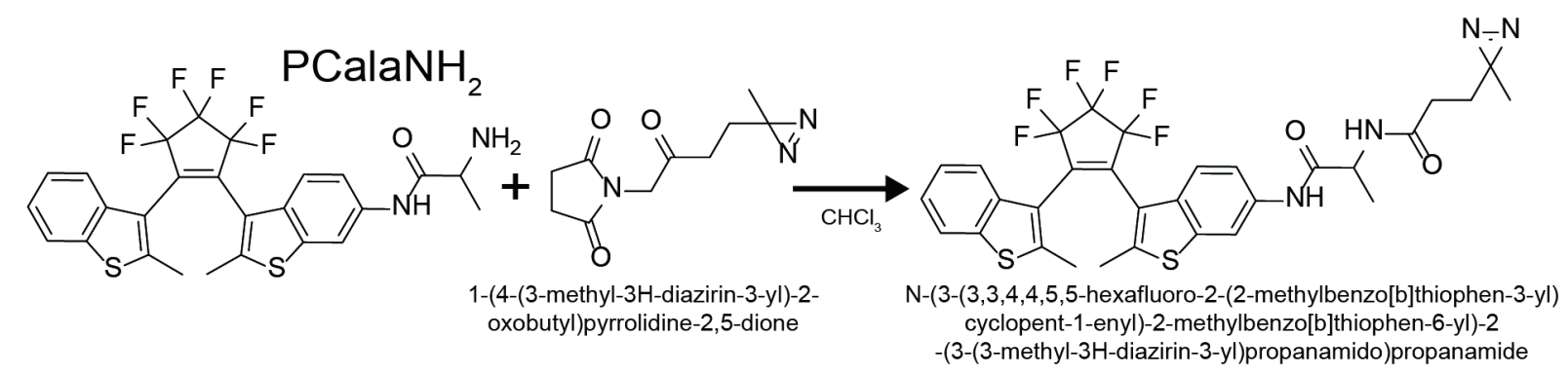

Scheme 13: Synthesis of photoactivatable cross-linking photochrome.

The primary amine of the $\mathrm{PCalaNH}_{2}$ can react with the NHS-ester of the commercial linker (SDA, Thermo\#26167). The reaction occurs in 90 minutes at RT, though it must be protected from light exposure throughout due to the reactivity of the diazirine group to UV. The final product is yellow and crystalline $\left(\mathrm{R}_{\mathrm{f}}=0.30,1: 1\right.$ cyclohexane: THF; yield, $\left.90 \%\right)$.

\subsection{Polymer Synthesis}

\subsubsection{Preparation of Photochromic Polymers}

The PMA backbone used in this work was acquired from Sigma-Aldrich and had an average of 40 succinic anhydride monomer groups. The principal advantage of PMA backbones is the straightforward functionalization. The synthesis of the photochromic comb-link amphiphilic polymer could be realized as a one-pot reaction. It is important to note that the conjugation of the aromatic amine $\left(\mathrm{PCmNH}_{2}\right)$ is inferior to that of the aliphatic amines $\left(\mathrm{PCalaNH}_{2}, \ldots, \mathrm{PCadoNH}_{2}\right)$ due to the nucleophilicity of the amine as well as steric hindrance. ${ }^{17}$ It is assumed that the dodecylamine used to 
create the comb-like structure reacts to completion. Independently of the PC utilized the polymer preparation was similar.

The PMA polymer was introduced into a dry $10 \mathrm{~mL}$ flask, and the chosen PC was added in excess to the desired conjugation percentage, as an anhydrous THF solution. A solution of dodecylamine (anhydrous THF) was added so as to conjugate $25 \%$ of the succinic anhydride monomers. Initially a cloudy yellowish solution is obtained and within 15-20 min of dodecylamine addition the PMA solubilizes and the solution turns clear. The reaction was left overnight at $60{ }^{\circ} \mathrm{C}$. In the morning an additional portion of dodecylamine, $50 \%$ of the anhydride equivalents, was added and allowed to react for $>6 \mathrm{~h}$.
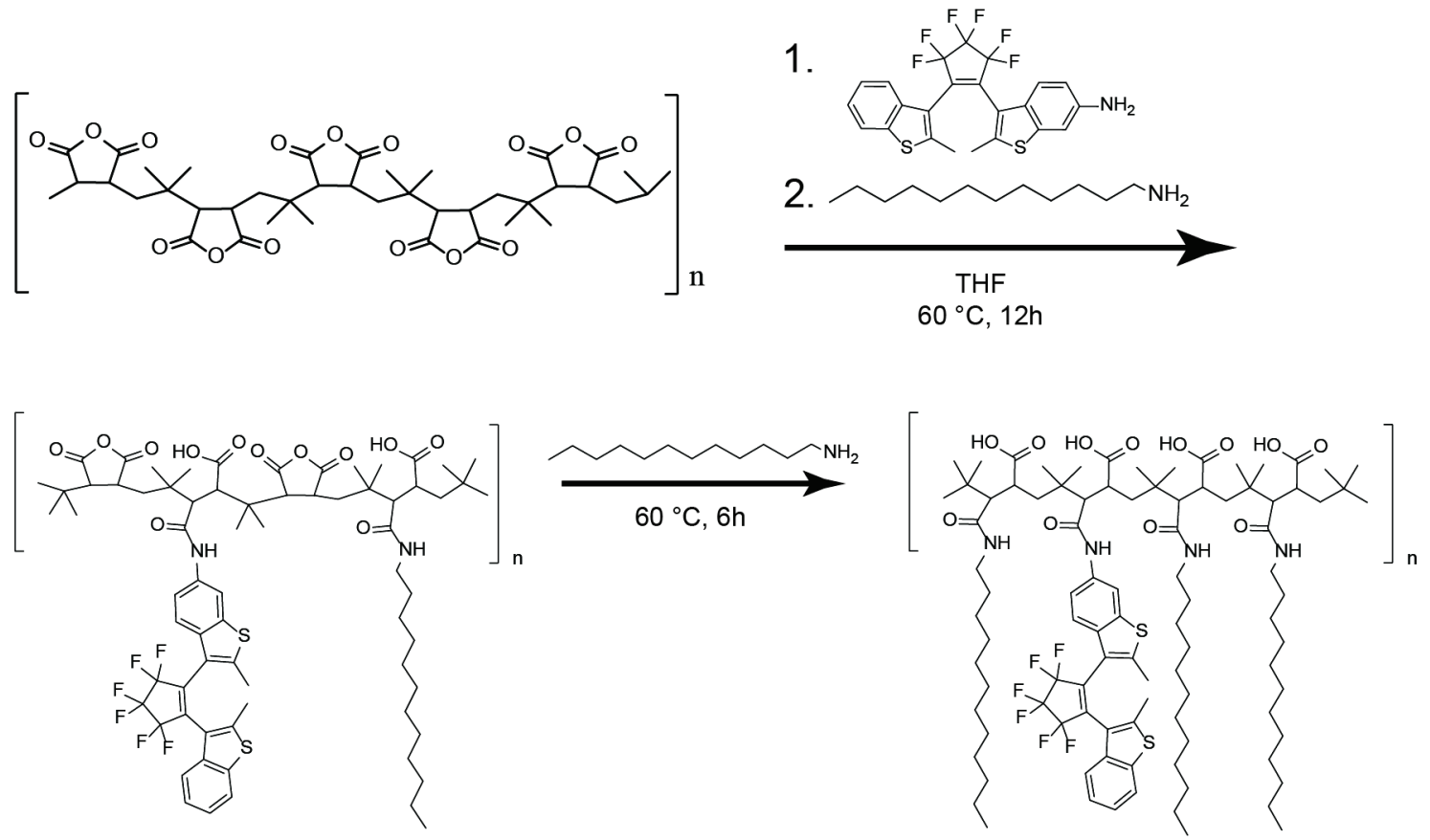

Scheme 14: Preparation of photochromic amphiphilic comb polymer.

This protocol, with the 2-part addition of dodecylamine, was a slight modification of that found in the literature. ${ }^{19} \mathrm{I}$ found that adding the initial dodecylamine improved conjugation of the PC, as compared to PC alone or PC after complete addition of dodecylamine. This may be due to the increased solubility of the PMA.

The excess PC was calculated according to whether the PC had an aromatic amine or aliphatic amine. I utilized the nomenclature proposed by the Parak group according to which modifications to the PMA backbone are represented as percentages of anhydride rings coupled with the added molecules. ${ }^{10}$ As an example, a polymer in which $1 \%$ of the anhydride rings are modified with the PC group and $75 \%$ with dodecylamine chains should be identified as PMA 1PC 75C12, where C12 is shorthand for dodecylamine. For aromatic amines a 8-10 fold excess was recommended, while for aliphatic amines a 1.5-3.0 fold excess. The dodecylamine conjugation was assumed to be stoichiometric and was designed to be $75 \%$. 
Purification consisted of drying the polymer, dissolving in $\mathrm{CHCl}_{3}$, followed by separation from unreacted reagents using a Sephadex LH-20 size-exclusion column.

A series of different photochromic polymers were synthesized and characterized (as described in the next section). Table 4 provides a complete list with short descriptions.

Table 4: Description of prepared polymers.

\begin{tabular}{|c|c|}
\hline Polymer & Description \\
\hline PMA $75 \mathrm{C} 12$ & $\begin{array}{l}\text { Amphiphilic comb-polymer without photochromes. Often used as a control } \\
\text { polymer }\end{array}$ \\
\hline PMA 10PC & $\begin{array}{l}\text { A polymer containing only } \mathrm{PCmNH}_{2} \text {. The polymer is photochromic in organic } \\
\text { solvents and buffer, but is not able to coat NPs. }\end{array}$ \\
\hline PMA [0.4-1.7]PC 75C12 & $\begin{array}{l}\text { Amphiphilic comb-polymer with different } \mathrm{PCmNH}_{2} \text { concentrations. The } \\
\text { polymers are photochromic and can coat NPs. Due to the low PC concentration, } \\
\text { the experiments with these polymers are not reported. }\end{array}$ \\
\hline PMA 2PC 75C12 & Amphiphilic comb-polymer with $2 \% \mathrm{PCmNH}_{2} . \mathrm{See} \mathrm{Ref}^{8}$ \\
\hline PMA 6PC 75C12 & Amphiphilic comb-polymer with $6 \% \mathrm{PCmNH}_{2}$. See $\mathrm{Ref}^{8}$ \\
\hline PMA 6PCala 75C12 & Amphiphilic comb-polymer with $6 \% \mathrm{PCalaNH}_{2}$. \\
\hline PMA 4PCahx 75C12 & Amphiphilic comb-polymer with $4 \% \mathrm{PCah} \mathrm{NH}_{2}$. \\
\hline PMA 8PCaoc 75C12 & Amphiphilic comb-polymer with $8 \% \mathrm{PCaocNH}_{2}$. \\
\hline PMA 4PCado 75C12 & Amphiphilic comb-polymer with 4\% $\mathrm{PCadoNH}_{2}$. \\
\hline PMA 30PCado 70C12 & $\begin{array}{l}\text { Amphiphilic comb-polymer with } 30 \% \mathrm{PCadoNH}_{2} \text {. The polymer is photochromic } \\
\text { in organic solvents and buffer, but is not able to coat NPs. }\end{array}$ \\
\hline
\end{tabular}

\subsubsection{Polymer Characterization}

The principal characterization is the determination of PC concentration on the polymers. The simplest method for this purpose was absorbance spectroscopy. I assumed that the absorption and photoconversion properties of the conjugated PC are the same as for PCmonomer (Section 1.2.2.).

A known amount of polymer, in the $0.3-1.0 \mathrm{mg} / \mathrm{ml}$ range, was dissolved in an organic solvent that permits efficient photoconversion ( $\mathrm{THF}, \mathrm{CHCl}_{3}$ ). The spectra in the open form and the photostationary closed form $\left(\alpha_{P S}=22 \%\right)$ were obtained. Using the difference in extinction coefficients at $545 \mathrm{~nm}$ the concentration of closed PC in solution was obtained and thus the total concentration of PC. Assuming that the reaction with the dodecylamine was near stoichiometric, the molar concentration of the polymer was calculated so as to obtain the fraction of PC in the polymer. Using this methodology all the polymers in Table 4 were assigned their PC concentration. 


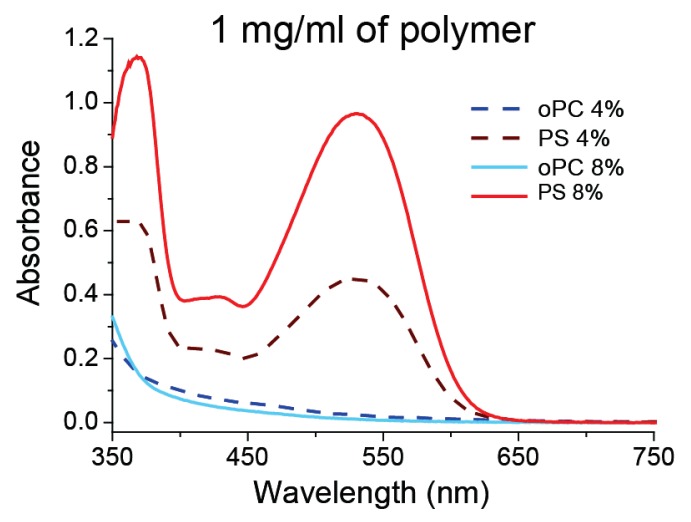

Figure 4: Absorbance spectra of two representative polymers with different PC concentrations.

NMR studies of PMA functionalization can be found in the literature. ${ }^{20}$ Using ${ }^{1} \mathrm{H}$ NMR the following signals were used to determine alkyl modification of PMA; $\mathrm{CDCl}_{3}, 0.88 \mathrm{ppm}$ (-CH3 dodecylamine), $0.75-1.10 \mathrm{ppm}$ (-CH3 butylene groups), 1.20-1.25 ppm (-CH2- dodecylamine), 2.7$3.6 \mathrm{ppm}$ (-CH2- in alpha to amide or carboxyl groups). As can be seen in Figure 5 the peaks are very broad and the quantification is complicated.

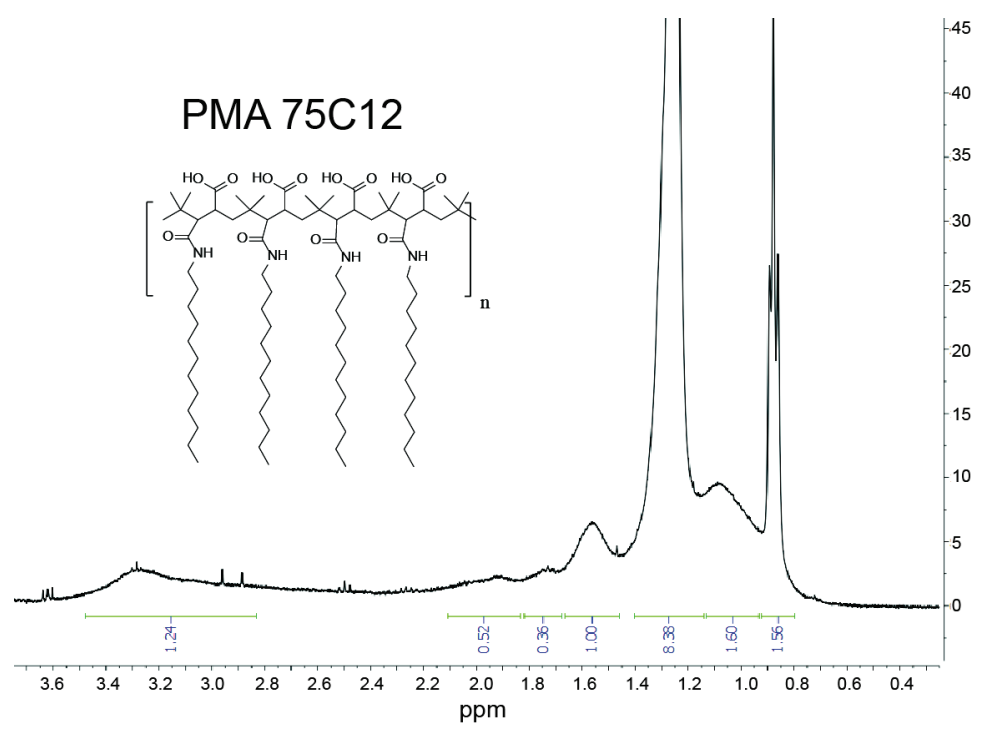

Figure 5: ${ }^{1} \mathrm{H}$ NMR of PMA $75 \mathrm{C} 12$.

I attempted to quantify the PC concentration using the aromatic region of the NMR spectra. At high concentrations, for example PMA 30PCado 70C12 (see Figure 6), the PC signals can be observed, yet quantification remains challenging. Samples with lower concentration of PC were impossible to characterize with this method. 


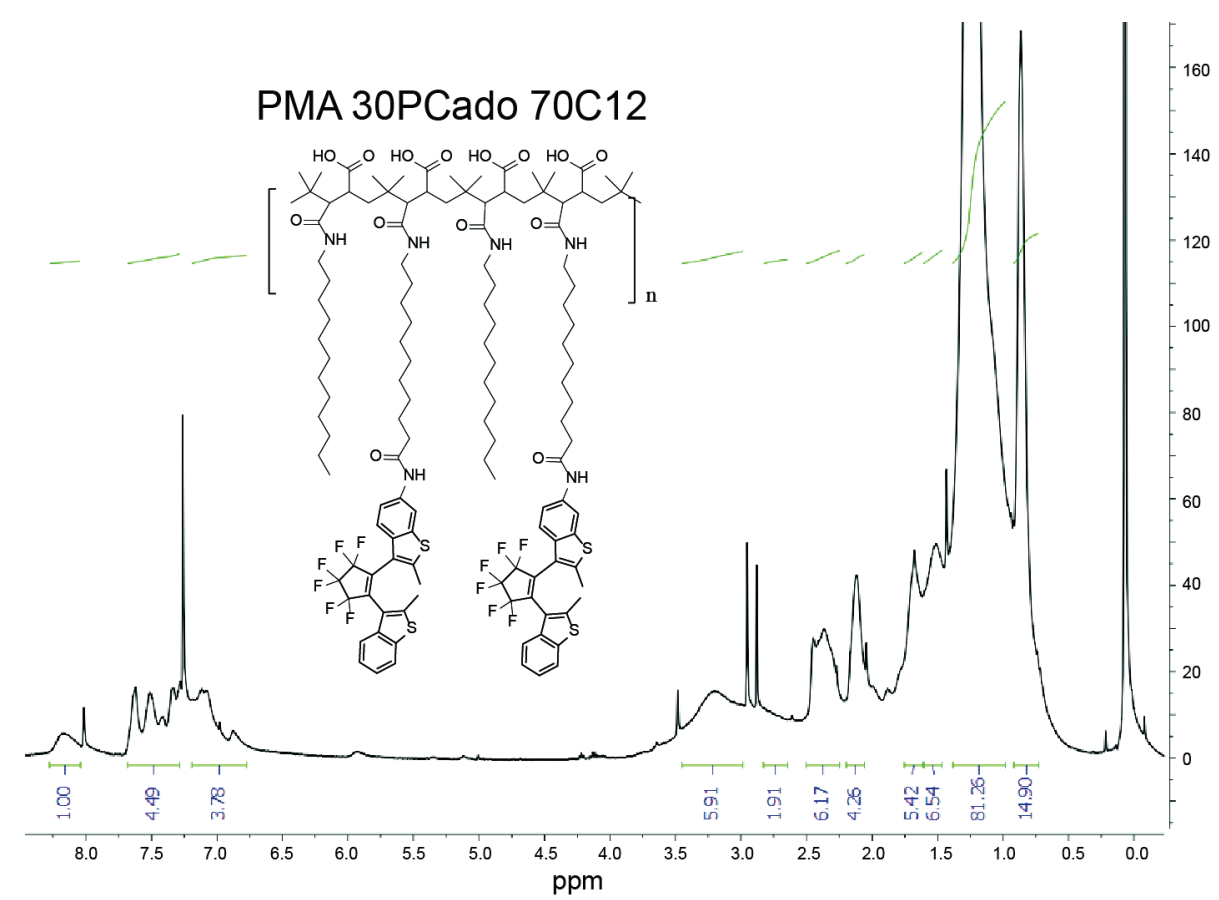

Figure 6: ${ }^{1} \mathrm{H}$ NMR of PMA 30PCado $70 \mathrm{C} 12$

In a similar manner XPS (X-ray photoelectron spectroscopy) was attempted with the hope of quantifying the ratio of fluor to oxygen and nitrogen. Silica substrates were covered with PMA 2PC 75C12 and PMA 6PC 75C12 and then the XPS was realized in the labs of Prof. Dr. Hans Hofsäß (2nd Institute of Physics, University of Göttingen). Unfortunately, although the detection limit is in the parts per thousand, the quantification limit is $>1$ Atomic \% for any atom. A fluor signal could be detected, but even using the PMA 6PC 75C12 the amount of fluor was not high enough for quantification.

\subsection{Bifunctional Polymer Synthesis}

PMA backbones can be modified with more than one functional group. While the PC groups were hydrophobic moieties the second modifications were hydrophilic groups, chosen with the intent that they would be exposed to the exterior when coated on NPs. The functionalization was divided into two main groups: fluorescent dyes or targeting molecules. The polymer can be modified in a postsynthetic step using the carboxyl groups and an amine bearing molecule through couplers such as EDC or DCC [N,N'-Dicyclohexylcarbodiimide]. In Chapter 5 I will show that though this is feasible it also presents various drawbacks, such as undesired crosslinking and aggregation.

\subsubsection{Bifunctionalization with PC and a Fluorescent Dyes}

The fluorescent dyes selected where chosen so as to be exposed outwards, i.e. towards the aqueous medium, when formed as polymersomes or coated on NPs. The fluorescent probes had to contain an amine capable of reacting with the anhydrides, so Lucifer Yellow (LY) Cadaverine and AlexaFluor $647(\mathrm{Al})$ Cadaverine were selected. Certain simple modifications of the above reported 
polymer syntheses were required due to the different solvent solubilities of the dyes. The principal modification can be summarized as the need to add the fluorescent dye as a DMSO:DMF solution, before the addition of any other reagent to the PMA backbone. After $90 \mathrm{~min}$, the selected PC and initial dodecylamine were added.

The first polymer obtained was PMA 7PCahx 75C12 0.3Al. For every 1000 succinic anhydride monomers there were 750 dodecylamine chains, 70 PCahx molecules, and 3 Alexa647. This polymer was characterized in a manner similar to that used on the simple photochromic polymers, with the additional determination of Alexa647 coming from the absorbance at $650 \mathrm{~nm}$. This polymer was photochromic and able to coat NPs. When coated on a QD the Alexa647 served as an internal reference for the modulation of the QD fluorescence, creating ratiometric possibilities. For details see Section 3.3 and reference ${ }^{21}$.

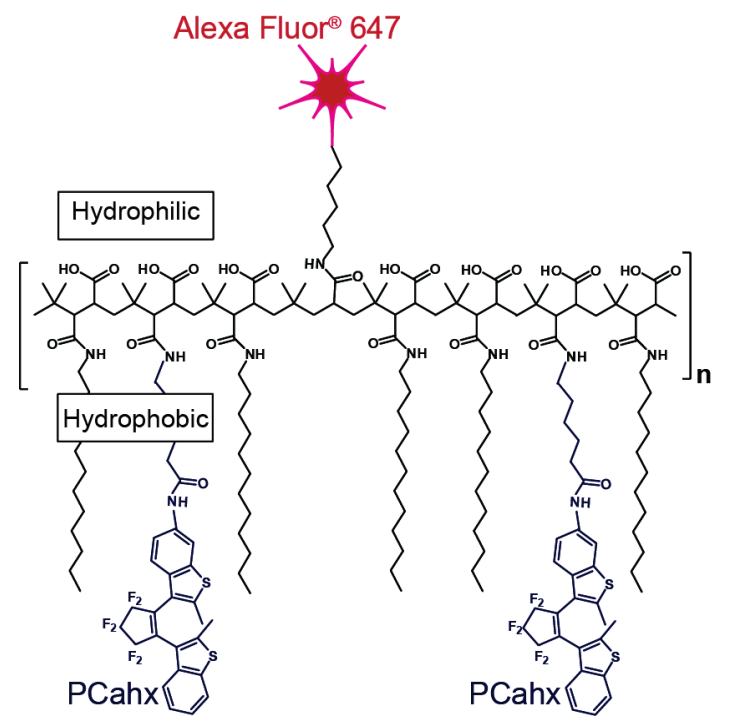

Scheme 15: Schematic of photochromic amphiphilic comb polymer doped with Alexa647

The second polymer was PMA 9PCala 75C12 2.5LY, containing 90 PCala molecules, 750 dodecylamine chains, and 25 Lucifer Yellow for every 1000 original monomers. The PC was determined in the same manner as before, while the LY concentration was calculated from the absorbance at $430 \mathrm{~nm}$ after correcting for the contributions of the PC and scattering by the polymer. The polymer acted as a FRET pair by itself, the LY emitting with a broad peak around $530 \mathrm{~nm}$ a wavelength at which the PC can act as a good FRET acceptor. This polymer was characterized in three manners: as individual polymer strands, as a polymersome, and coated on a QD. The properties of the polymer and the FRET pair changed drastically according to the nanostructure adopted by the polymer. For details see Section 2.1 and reference ${ }^{22}$. 


\subsubsection{Bifunctionalization with PC and Targeting Molecules}

The Biotin-Streptavidin binding pair is often used in biological applications, such as purification or targeting, since it presents an extremely low dissociation constant $\left(\mathrm{Kd} \sim 10^{-15} \mathrm{M}\right)$. In addition biotin is a small molecule $(244.3 \mathrm{~g} / \mathrm{mol})$ which presents no toxicity to living cells even at high concentrations. A commercial PEG (polyethylene glycol) reagent was purchased, which had a primary amine on one end and a biotin molecule on the other. The reagent, known as Amine-PEG11-Biotin (Pierce\#26136), was conjugated to the polymer through the amine group. PEG increases water solubility of the single strand polymer and of polymer coated NPs and the longer linker ensures that the biotin is exposed, avoiding steric interference from the polymer or NP surface.

The content of PEG was quantified using ${ }^{1} \mathrm{H}$ NMR spectroscopy. A peak at $3.65 \mathrm{ppm}$ can be assigned to the methyl groups of the PEG linker. This peak was integrated and compared to the 1.25 ppm integration corresponding to the dodecylamine methyl groups. Assuming 75\% for $\mathrm{C} 12$ the obtained value is $1.3 \%$ for the PEG-biotin linker.

PMA 6PCala 75C12 1.3PEG-Bt

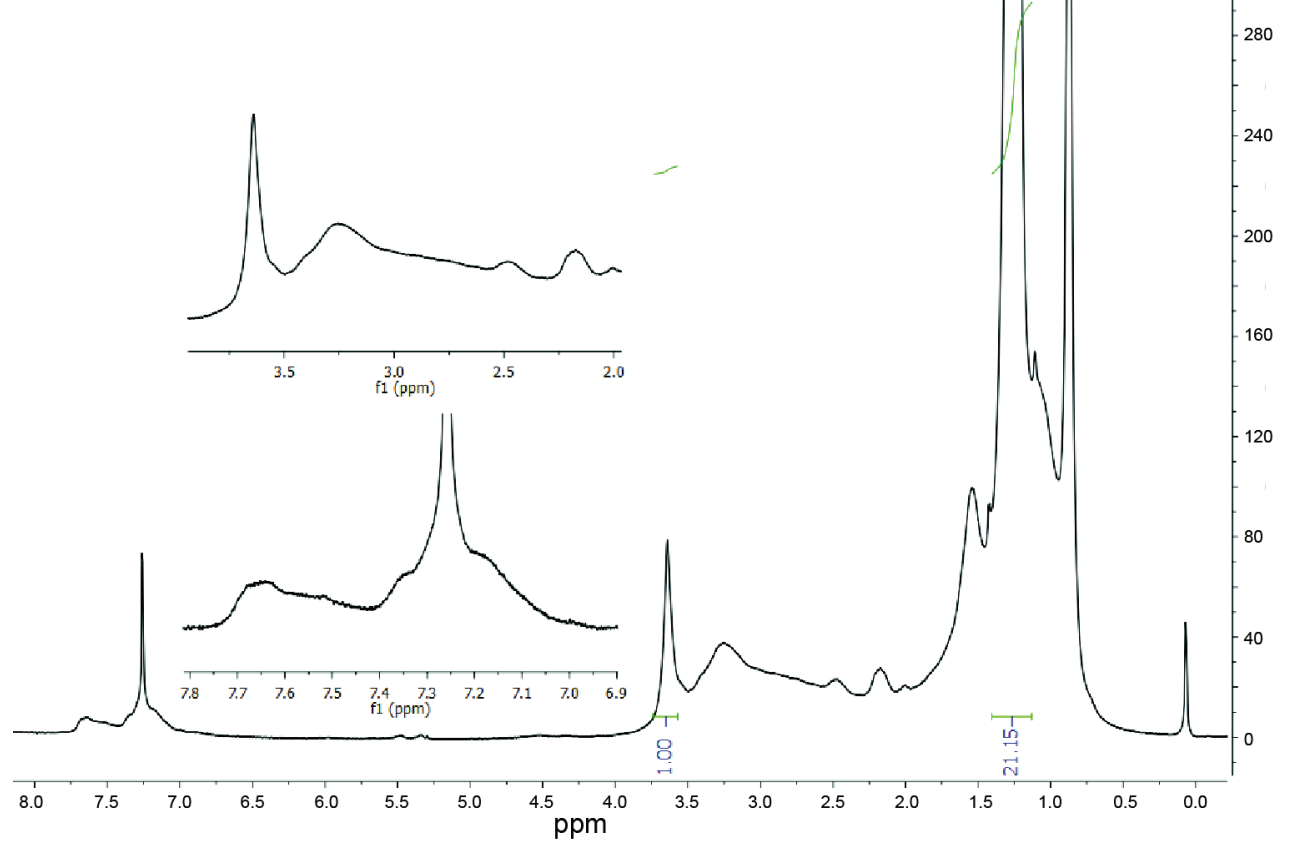

Figure 7: ${ }^{1} \mathrm{H}$ NMR of PMA 6PCala 75C12 1.3PEG-Bt

The polymer was photochromic and could coat QDs. It will be shown in Chapter 5 that the QDs coated with the biotin functionalized polymer could be used for targeting the prepared nanoprobes to biological molecules of interest. 


\section{References}

1. Perpète, E. A.; Maurel, F.; Jacquemin, D. TD-DFT Investigation of Diarylethene Dyes with Cyclopentene, Dihydrothiophene, and Dihydropyrrole Bridges. J. Phys. Chem. A 2007, 111, 5528-5535.

2. Tian, H.; Yang, S. Recent Progresses on Diarylethene Based Photochromic Switches. Chem. Soc. Rev. 2004, 33, 85-97.

3. Goldberg, A.; Murakami, A.; Kanda, K.; Kobayashi, T.; Nakamura, S.; Uchida, K.; Sekiya, H.; Fukaminato, T.; Kawai, T.; Kobatake, S.; Irie, M. Rotational Isomerization of Dithienylethenes: A Study on the Mechanism Determining Quantum Yield of Cyclization Reaction. J. Phys. Chem. A 2003, $107,4982-4988$.

4. Sysoiev, D.; Yushchenko, T.; Scheer, E.; Groth, U.; Steiner, U. E.; Exner, T. E.; Huhn, T. Pronounced Effects on Switching Efficiency of Diarylcycloalkenes Upon Cycloalkene Ring Contraction. Chem. Commun. 2012, 48, 11355-11357.

5. Irie, M.; Mohri, M. Thermally Irreversible Photochromic Systems. Reversible Photocyclization of Diarylethene Derivatives. J. Org. Chem. 1988, 53, 803-808.

6. Irie, M. Photochromism of Diarylethene Single Molecules and Single Crystals. Photochem. Photobiol. Sci. 2010, 9, 1535-1542.

7. Luo, Q. F.; Cheng, H.; Tian, H. Recent Progress on Photochromic Diarylethene Polymers. Polym. Chem. 2011, 2, 2435-2443.

8. Díaz, S. A.; Menendez, G. O.; Etchehon, M. H.; Giordano, L.; Jovin, T. M.; Jares-Erijman, E. A. Photoswitchable Water-Soluble Quantum Dots: pcFRET Based on Amphiphilic Photochromic Polymer Coating. ACS Nano 2011, 5, 2795-2805.

9. Pellegrino, T.; Manna, L.; Kudera, S.; Liedl, T.; Koktysh, D.; Rogach, A. L.; Keller, S.; Rädler, J.; Natile, G.; Parak, W. J. Hydrophobic Nanocrystals Coated with an Amphiphilic Polymer Shell: A General Route to Water Soluble Nanocrystals. Nano Lett. 2004, 4, 703-707.

10. Fernández-Argüelles, M. T.; Yakovlev, A.; Sperling, R. A.; Luccardini, C.; Gaillard, S.; Sanz Medel, A.; Mallet, J.-M.; Brochon, J.-C.; Feltz, A.; Oheim, M.; Parak, W. J. Synthesis and Characterization of Polymer-Coated Quantum Dots with Integrated Acceptor Dyes as FRET-Based Nanoprobes. Nano Lett. 2007, 7, 2613-2617.

11. Jańczewski, D.; Tomczak, N.; Han, M.-Y.; Vancso, G. J. Synthesis of Functionalized Amphiphilic Polymers for Coating Quantum Dots. Nat. Protoc. 2011, 6, 1546-1553.

12. Mattern, D. L. Direct Aromatic Periodination. J. Org. Chem. 1984, 49, 3051-3053.

13. Giordano, L. Síntesis De Diheteroariletenos Fotocrómicos Y Su Aplicación Como Aceptores En Transferencia De Energía. Universidad de Buenos Aires, Buenos Aires, 2006.

14. Satoh, T.; Suzuki, S. Reduction of Organic Compounds with Sodium Borohydride-Transition Metal Salt Systems (1) Reduction of Organic Nitride, Nitro and Amide Compounds to Primary Amines. Tetrahedron Lett. 1969, 52, 4555-4558.

15. Irie, M.; Miyatake, O.; Uchida, K. Blocked Photochromism of Diarylethenes. J. Am. Chem. Soc. 1992, $114,8715-8716$.

16. Giordano, L.; Jovin, T. M.; Irie, M.; Jares-Erijman, E. A. Diheteroarylethenes as Thermally Stable Photoswitchable Acceptors in Photochromic Fluorescence Resonance Energy Transfer (pcFRET). $J$. Am. Chem. Soc. 2002, 124, 7481-7489.

17. Hu, G. H.; Lindt, J. T. Amidification of Poly(Styrene-Co-Maleic Anhydride) with Amines in Tetrahydrofuran Solution: A Kinetic Study. Polym. Bull. 1992, 29, 357-363. 
18. Massolini, G.; Calleri, E. Survey of Binding Properties of Fatty Acid-Binding Proteins Chromatographic Methods. J. Chromatogr. B 2003, 797, 255-268.

19. Yakovlev, A. V.; Zhang, F.; Zulqurnain, A.; Azhar-Zahoor, A.; Luccardini, C.; Gaillard, S. p.; Mallet, J.-M.; Tauc, P.; Brochon, J.-C.; Parak, W. J.; Feltz, A.; Oheim, M. Wrapping Nanocrystals with an Amphiphilic Polymer Preloaded with Fixed Amounts of Fluorophore Generates FRET-Based Nanoprobes with a Controlled Donor/Acceptor Ratio. Langmuir 2009, 25, 3232-3239.

20. Jańczewski, D.; Tomczak, N.; Khin, Y. W.; Han, M.-Y.; Julius Vancso, G. Designer Multi-Functional Comb-Polymers for Surface Engineering of Quantum Dots on the Nanoscale. Eur. Polym. J. 2009, 45, 3-9.

21. Díaz, S. A.; Giordano, L.; Jovin, T. M.; Jares-Erijman, E. A. Modulation of a Photoswitchable DualColor Quantum Dot Containing a Photochromic FRET Acceptor and an Internal Standard. Nano Lett. 2012, 12, 3537-3544.

22. Diaz, S. A.; Giordano, L.; Azcarate, J. C.; Jovin, T. M.; Jares-Erijman, E. A. Quantum Dots as Templates for Self-Assembly of Photoswitchable Polymers: Small, Dual-Color Nanoparticles Capable of Facile Photomodulation. J. Am. Chem. Soc. 2013, 135, 3208-3217. 


\section{CHAPTER 2: CHARACTERIZATION OF POLYMER BOUND PCFRET PAIR}

\section{Introduction}

A list of the photochromic amphiphilic PMA based polymers that were prepared and characterized during my research can be found in Section 1.4. Though the literature demonstrated the use of the PMA based polymers in coating NPs, ${ }^{1,2}$ a detailed step-by-step characterization had not been reported. My goals included determining how the properties of the photochromic FRET acceptor were modified according to the environment and how the self-assembly of the polymers varied in organic solvent, buffers, and coated onto NPs. To do this I required a polymer that contained a second reporting probe along with the PC.

In 2002 Giordano et $a l^{3}$ first reported on the use of diheteroarylethenes as a pcFRET acceptor for Lucifer Yellow (LY). The initial study provided in-depth analysis, including photoconversion and kinetic parameters, of the directly linked donor and acceptor maintained in organic solvents, allowing for the efficient photoswitching of the PC. The available information made the pcFRET a good reporter unit selection. The strategy adopted in this thesis was to conjugate the pcFRET pair to PMA, thereby creating an amphiphilic scaffold capable of overcoming the organic solvent requirement.

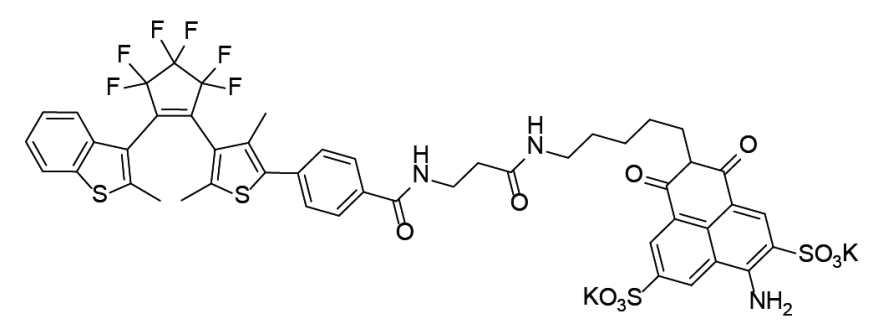

Scheme 1: pcFRET pair from Giordano et al (2002). ${ }^{3}$

In combination with the above stated goals, there existed interest in finding new constructs for imaging purposes. Micelles and polymer vesicles (polymersomes) have been shown to function as imaging agents in cells and animal models. ${ }^{4,5}$ Polymersomes prepared with the polymer containing the pcFRET pair would add a valuable photoswitchable property to preexisting constructs. Of even greater interest was the templating of the polymer on a QD, creating a new, improved probe. I first present the properties of the polymer in organic solvents and then proceed to the studies of the polymersome and QD templated probe in Section 2.2 and 2.3.

\subsection{Photoswitchable Polymer in Organic Solvents}

A PMA polymer backbone was utilized to create the photomodulatable polymer by conjugation with dodecylamine, $\mathrm{PCalaNH}_{2}$ molecules acting as FRET acceptors, and Lucifer Yellow cadaverine as the fluorescent donor. All three constituent molecules carry primary amine groups to 
conjugate to PMA. The short linker on the PC increases conjugation to the polymer while minimizing the distance from the LY on the polymer backbone. The chosen polymer was PMA 9PCala 75C12 2.5LY, this designation indicates the presence of 90 PCala molecules, 750 dodecylamine chains, and 25 Lucifer Yellow for every 1000 original monomers (see Scheme 2). Considering that each polymer chain contained $\sim 40$ monomers, each chain on average had $1 \mathrm{LY}$ and 3.6 PCala. If a random (Poissonian) distribution of the dyes is assumed, $37 \%$ of polymer chains had no LY, while there were less than $2.5 \%$ of polymer chains without PCala. Thus, $<1 \%$ of polymer chains should have a LY and no PC. The characterization of the photochrome and polymer can be found in Section 1.3 and 1.4 respectively.
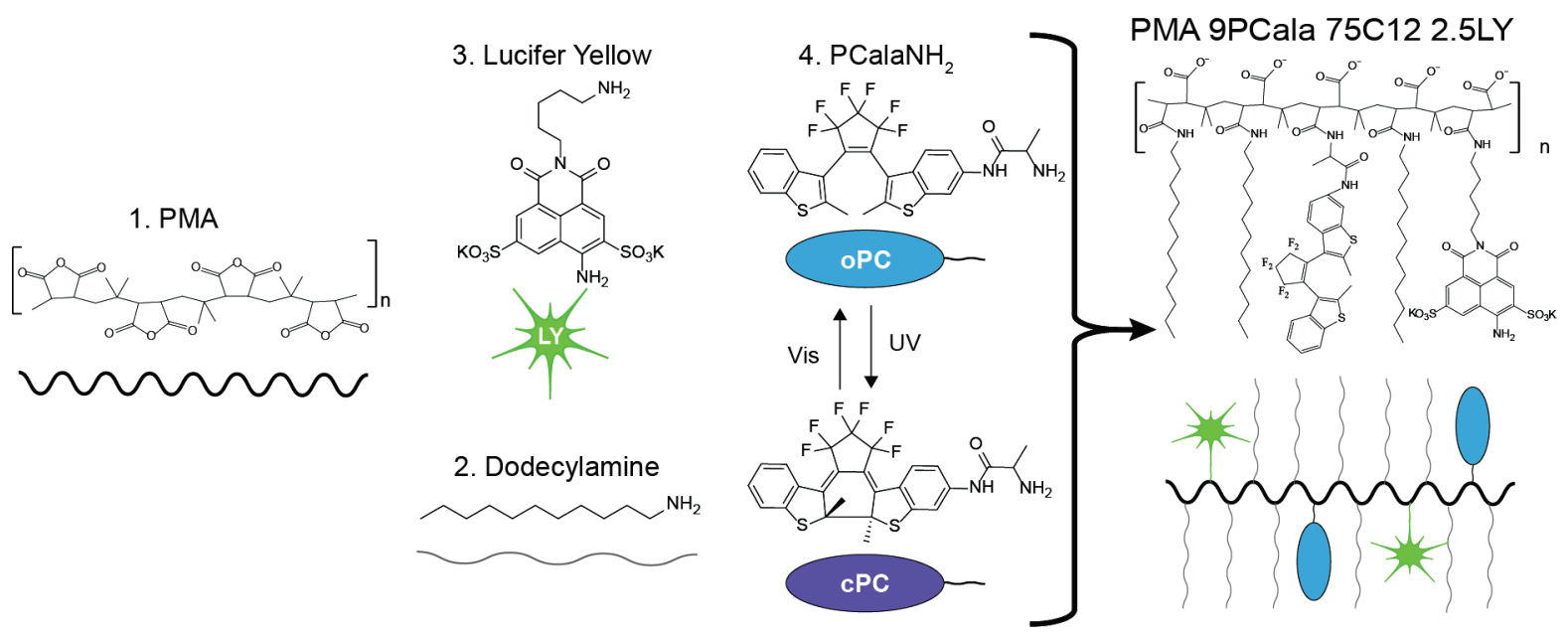

Scheme 2: Components and final preparation of PMA 9PCala 75C12 2.5LY.

To help understand the analysis, characterizations, and comparisons presented from here on, a photophysical description of PC is provided. The PC can exist in two states, the open form (oPC) and the photocyclized closed form (cPC), obtained upon irradiation of the oPC with UV light. $^{6}$ Back conversion to the oPC form in $\sim 100 \%$ yield is accomplished by exposure to green light which is only absorbed by cPC. In contrast, the formation of $\mathrm{cPC}$ is never $100 \%$ due to the photoreversal that occurs upon excitation of $\mathrm{cPC}^{6}{ }^{6}$ As will be seen the $\mathrm{cPC}$ excitation can be direct due to UV irradiation or due to energy transfer. Irradiation with UV light eventually reaches a photostationary state (PS) that depends solely on the wavelength (but not the intensity) of irradiation and the local environment of the PC groups. ${ }^{7}$ In an organic solvent and with minimal FRET, exposure to UV light ( $340 \pm 10 \mathrm{~nm}$ ) led to a fractional $\mathrm{cPC}$ content in the photostationary state, $\alpha_{\mathrm{PS}}=\mathrm{cPC} /(\mathrm{cPC}+\mathrm{oPC})$, of 0.22 . The concentration of total PC was determined from $\mathrm{Abs}_{540}$, the $\alpha_{\mathrm{PS}}$, and the known differences in extinction coefficients. The PC and LY appeared not to be modified by its conjugation to the polymer. From the $\alpha_{\mathrm{PS}}$ of 0.22 , a $\sim 1-1.25$ maximum ratio of acceptors (cPC) to donors (LY) was determined. The design goal was a 1:1 stoichiometry, but the variability of synthesis resulted in the PMA 9PCala 75C12 2.5LY being the preferred polymer of study. Unless otherwise indicated, all subsequent mention of polymers in this chapter will be of this dual functionalized polymer. 


\subsubsection{Single Polymer Strands in Solution}

To begin the step-by-step analysis of the system it was required that the nano-conformation of the polymer in each system be determined. Do individual polymer chains interact in dilute solutions with "good solvents" (i.e. THF, $\mathrm{CHCl}_{3}, \mathrm{AcOEt}$ )? To determine this I utilized time-resolved fluorescent anisotropy.

The first step was estimating the characteristics of the polymer using Gaussian Chain Statistics Theory. ${ }^{8}$ The polymer was represented as a real chain in a good solvent, presenting excluded volume effects. This means that polymer segments take up physical space that cannot be occupied by other segments. This representation requires an expansion factor $>1$ to obtain the actual dimensions. I was principally interested in the mean-squared radius of gyration of a chainlike polymer which is calculated in Equation 1.

$$
\left\langle\mathrm{S}_{\mathrm{g}}^{2}\right\rangle=\mathrm{Nl}^{2} \alpha^{2} / 6
$$

with $\mathrm{N}$, the chain length; 1 , the segment lattice length; and $\alpha$, the expansion factor accounting for excluded volume effects. Since $\mathrm{CHCl}_{3}$ was chosen as the solvent, the favorable polymer-solvent interactions and relative short length of the polymer chain results in $\alpha \approx 1.5{ }^{8}$ The parameters $\mathrm{N}=40$ and $1=0.52 \mathrm{~nm}$, lead to an estimate for $\left\langle\mathrm{S}_{\mathrm{g}}^{2}\right\rangle^{1 / 2}$ of $2.0 \mathrm{~nm}$.

A dilute $\mathrm{CHCl}_{3}$ solution of the polymer was examined by time-resolved fluorescent anisotropy of the LY fluorescence. The sample was irradiated to determine if the photocycling would have an effect on the polymer nanoconformation, but the obtained parameters did not change. The LY data was fit to a dual-decay model [Equations 2 and 3]. A wobble-in-a-cone model was selected, since it was believed that the comb-like structure of the polymer would limit the motional freedom of the dye, ${ }^{9}$ combined with a segmental-global rotational depolarization model for the whole polymer. ${ }^{10}$

$$
\begin{array}{ll}
r(t)=r_{o}\left[\left(1-A_{\infty}\right) e^{-t / \phi_{\text {dye }}}+A_{\infty}\right] e^{-t / \phi_{\text {polymer }}} & \text { Eq. [2] } \\
A_{\infty}=\frac{r_{\infty}}{r_{o}}=\left[\frac{1}{2}(1+\cos \theta) \cos \theta\right]^{2} & \text { Eq. [3] }
\end{array}
$$

From the fits the following parameters were obtained: initial anisotropy $\left(\mathrm{r}_{0}\right), 0.43 \pm 0.03$; residual anisotropy dye $\left(\mathrm{r}_{\infty}\right), 0.11 \pm 0.01$; cone half angle $(\theta), 51 \pm 2^{\circ}$; rotational correlation times $\left(\phi_{\text {dye }}\right.$, $\left.0.37 \pm 0.12 \mathrm{~ns} ; \phi_{\text {polymer }}, 5.5 \pm 0.2 \mathrm{~ns}\right)$. 


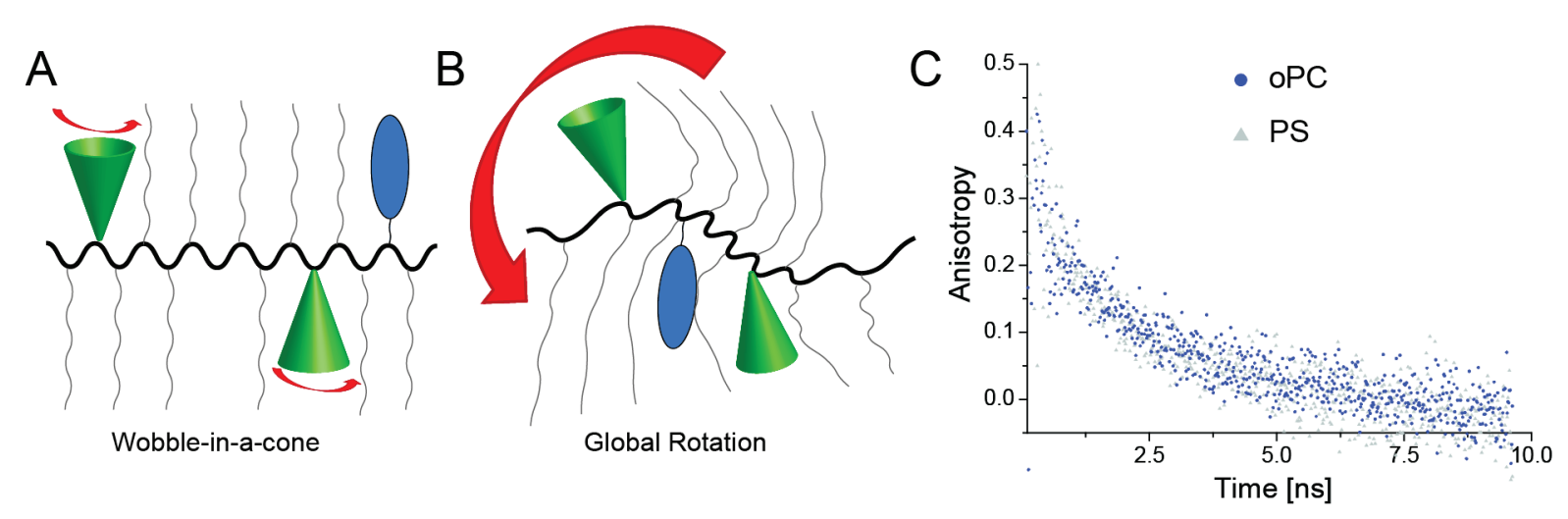

Scheme 3: A) Wobble-in-a-cone model B) Segmental-global rotational depolarization model C) Anisotropy curves of PMA 9PC 75C12 2.5LY in $\mathrm{CHCl}_{3}$.

With the obtained rotational time the Stokes-Einstein equation allows for the calculation of an equivalent sphere of rotation. ${ }^{8}$

$$
\phi_{\text {rot }}=\mathrm{V \eta} / \mathrm{kT}
$$

Where $\mathrm{k}$, Boltzmann constant; $\mathrm{T}=298 \mathrm{~K}$; and $\eta, \mathrm{CHCl}_{3}$ viscosity $=0.542 \mathrm{mPa} \bullet$ s. Once the volume is obtained a radius can be calculated to compare to the $\left\langle\mathrm{S}_{\mathrm{g}}^{2}\right\rangle^{1 / 2}$. The radius obtained from the anisotropy data was 2.1-2.2 nm, in good agreement with the $\left\langle\mathrm{S}_{\mathrm{g}}^{2}\right\rangle^{1 / 2}$ and consistent with PMA existing in $\mathrm{CHCl}_{3}$ as a freely draining dynamic chain with rather mobile pendant LY groups.

In fact this conformation - freely draining dynamic chain - is maintained in the presence of QDs, implying that in organic solvents in which the polymer and QD are soluble there is no selfassembly. This was tested by photoconverting two THF polymer solutions with the same concentration of polymer $(1.1 \mathrm{mg} / \mathrm{ml})$. One solution contained QDs (final concentration $90 \mathrm{nM}$ ) while the second was diluted with the same volume of THF. Chapter 3 will show that photochromic polymers bound to QDs have a decreased $\alpha_{\mathrm{PS}}$, yet that was not observed in organic solvents. Using the parameters presented in reference ${ }^{11}$, the $\alpha_{P S}$ of the QD solution should be in the range of 0.14 if the polymer was bound to the QD. The observed value was $\alpha_{P S}=0.21$, a trivial difference from 0.22 that can be explained by a combination of dilution error, trivial reabsorption, internal filter effects, and temporary interactions. 


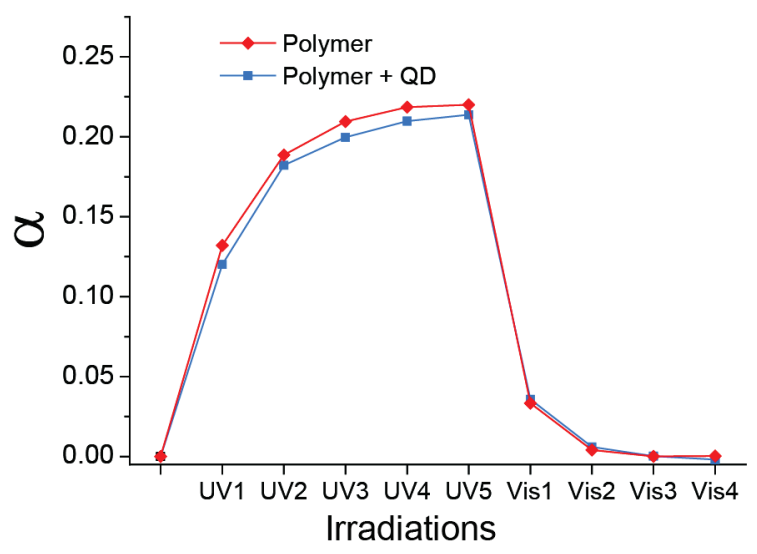

Figure 1: Change in conversion as a function of irradiation of a solution containing either photoswitchable polymer (red) or photoswitchable polymer and QDs (blue). Each point is after $60 \mathrm{~s}$ of corresponding irradiation (UV: $340 \mathrm{~nm}, 1 \mathrm{~mW} \mathrm{~cm}{ }^{-2}$; Vis:545 nm, $4.3 \mathrm{~mW} \mathrm{~cm}^{-2}$ ).

\subsubsection{Polymer Conjugated LY-PC as pcFRET Pair}

Knowing that the polymer chains do not interact in $\mathrm{CHCl}_{3}$ I proceeded to study the LY-PC FRET pair. The absorbance and fluorescence, both steady-state and time-resolved, of the solution was measured and can be observed in Figure 2. UV irradiation caused the photocyclization of the PC to the cPC form, changing the absorbance and quenching the fluorescence of the LY. The lifetime measurement demonstrated that the quenching mechanism was via FRET. ${ }^{12}$
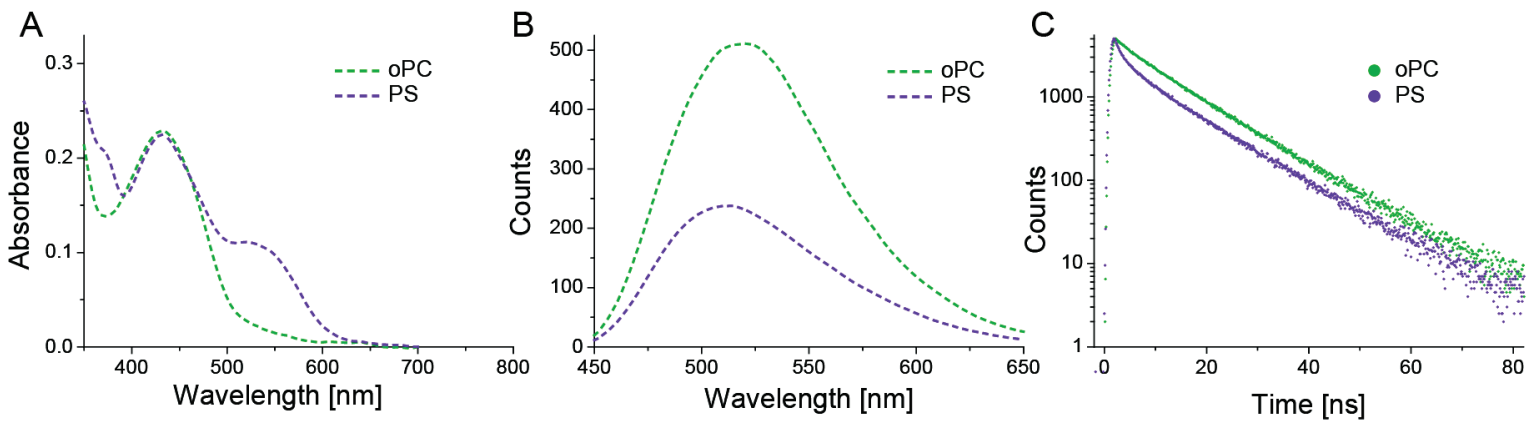

Figure 2: Spectra of PMA 9PC 75C12 2.5LY in $\mathrm{CHCl}_{3}$. Irradiation: $\mathrm{UV}, 340 \pm 10 \mathrm{~nm}$ (irradiance $1.1 \mathrm{~mW} \mathrm{~cm}^{-2}$ ); A) absorbance B) steady-state fluorescence C) lifetime fluorescence.

A considerable modulation was observed in both fluorescence measurements: in steady state fluorescence the quenching is $60 \%$ whereas in lifetime measurements it was $46 \%$ (Table 1). That is, the modulation in the lifetime measurement was smaller than the steady-state fluorescence quenching. This difference can be rationalized by invoking the existence of dark-states for the LY. At the photocyclization wavelength $(340 \mathrm{~nm})$ the LY has a low absorbance coefficient; such that the effect of FRET on the photoreversal (under these conditions) could be ignored even though the LY was a good FRET donor, resulting in a final $\alpha_{\mathrm{PS}}$ of 0.22 . 


\subsubsection{Kinetics of Photoconversion and Fluorescence Quenching of Polymer Chains}

The kinetics of photoconversion and quenching provided additional information for understanding the system. Figure 3 shows the photoconversion as a function of irradiation as well as the quenching as a function of the number of acceptor groups. The comparative analysis will be presented in Section 2.4. The conversion from $\mathrm{oPC}$ to $\mathrm{cPC}$ was fit with a mono-exponential function, and the apparent first order rate constant $\mathrm{k}_{\mathrm{oc}}^{\prime}$ was $58 \pm 2 \mathrm{~s}^{-1}$.
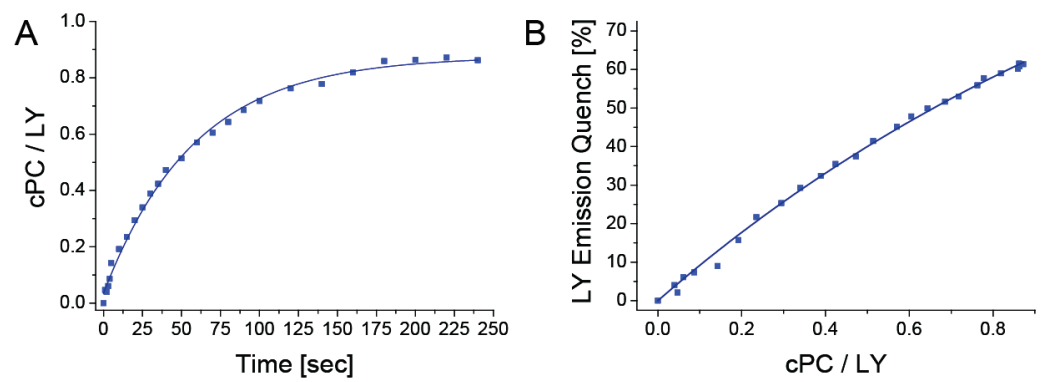

Figure 3: Photoconversion and quenching data (points) and corresponding fits (lines). (A) Graph of the evolution of $\mathrm{cPC} / \mathrm{LY}$ as a function of $\mathrm{UV}(1.1 \mathrm{~mW} \mathrm{~cm}$ ) irradiation time. Fit is monoexponential. (B) Quenching of LY fluorescence as a function of $\mathrm{cPC} / \mathrm{LY}$. Fit corresponds to Equation 5.

The case of the quenching as a function of the number of FRET acceptors (cPC) was a bit more complicated to fit. The FRET equation is given by,

$$
\begin{array}{ll}
\text { Quench } \%=100\left[\left(1-e^{-x}\right) \beta x+\sum_{k=1}^{m} \frac{e^{-x} x^{k}}{k !} \frac{k \gamma}{1+k \gamma}\right] & \text { Eq. [5] } \\
\gamma=\left(R_{o} / r_{D A}\right)^{6} & \text { Eq. [6] }
\end{array}
$$

in which, $\beta$ is a constant and $\mathrm{x}=\mathrm{cPC} / \mathrm{LY}$; in this particular instance it can also be represented as $\mathrm{cPC} /$ polymer. The first term, required for a satisfactory fit, accounts for a static quenching of LY by $\pi-\pi$ interactions such as those previously reported for this probe. ${ }^{13}$ Stacking would presumably occur preferentially with the $\mathrm{cPC}$ form, in view of the inherent intramolecular $\pi-\pi$ interaction featured by the parallel configuration of oPC and the reduced steric hindrance to $\pi-\pi$ interaction of the $\mathrm{cPC}$ form. This interaction may explain, in part, the LY dark-states. The second term of Eq. 1 represents LY-cPC FRET for the population of molecules, assuming a Poisson distribution of the cPC groups. As in the first term of Equation 1, quenching is assumed to occur only for $\mathrm{k} \geq 1$, implying that LYs would not sense $\mathrm{cPC}$ on a different polymer chain. The fit parameters $(\gamma=15 \pm 7, \beta=0.15 \pm 0.04)$ led to an estimated $\mathrm{r}_{\mathrm{DA}}$ of $2.6-3.1 \mathrm{~nm}$.

As a comparison, using the theoretical statistical model a mean square distance between donor and acceptor units on the same polymer strand was calculated. ${ }^{8}$

$$
\left\langle\mathrm{r}_{\mathrm{DA}}^{2}\right\rangle=\langle\mathrm{n}\rangle \mathrm{l}^{2} \alpha^{2} \quad \text { Eq. [7] }
$$

with 1 and $\alpha$ as in section 2.1.1, and where $\langle\mathrm{n}\rangle$ is the mean lattice separation of LY donors and cPC acceptors distributed randomly in the polymer; $\langle n\rangle=(N+1) / 3$. The value for $r_{D A}$ calculated in this manner was $2.9 \mathrm{~nm}$, in satisfactory agreement with the experimental results. 


\subsection{Photoswitchable Polymer as Polymersomes in Aqueous Solutions}

\subsubsection{Physical Characterization of Polymersomes}

The next step of the study was to transfer the polymer to an aqueous medium. PMA based amphiphilic polymers are known to form multichain polymersomes in buffer solutions. ${ }^{14}$ Thus, photomodulatable polymersomes were created by suspension of the polymer in $50 \mathrm{mM}$ sodium borate buffer (SBB), pH 9.
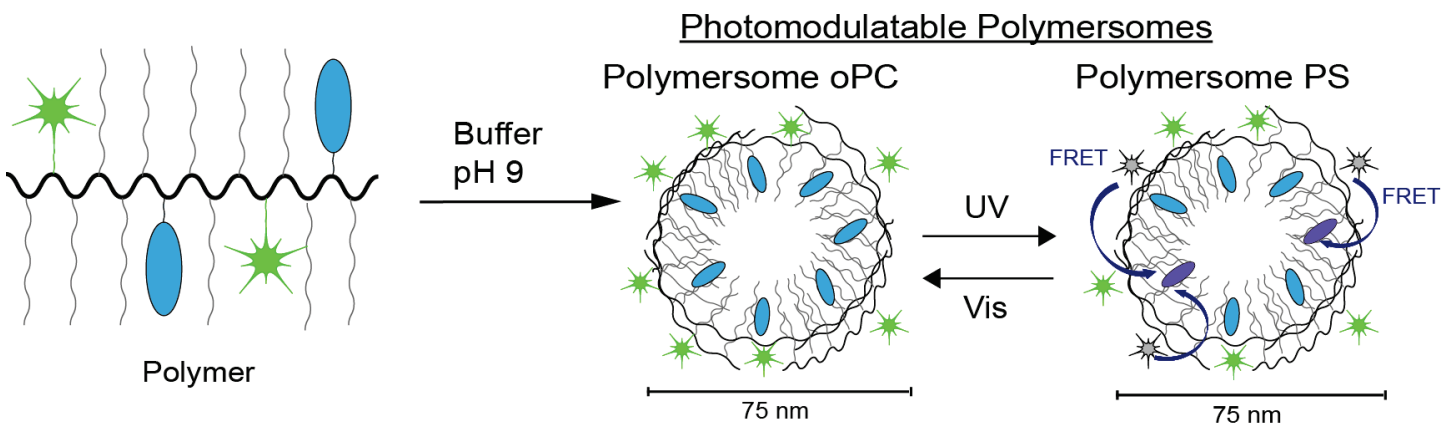

Scheme 4: Schematic representation of polymersomes and their photomodulation.

To confirm polymersome formation with the particular polymer I used Dynamic Light Scattering (DLS) and the Nanosight nanoparticle analysis system. The measured polymersomes had a broad diameter distribution of $75 \pm 35 \mathrm{~nm}$. The scattering contribution from the polymersomes is also evident in Figure 4.

\subsubsection{Spectral Characterization of Polymersomes}

The photophysical properties of both the LY and the PC changed dramatically from organic solvent to polymersome as can be seen in Table 1 . The larger QY, $\langle\tau\rangle_{\mathrm{amp}}$, and the blue-shifted emission of the first row are attributable to the aprotic non-polar environment provided by $\mathrm{CHCl}_{3}$, where apolar solvents inhibit certain non-radiative decay mechanisms. ${ }^{15}$ The larger value of $\mathrm{R}_{\mathrm{o}}$ in $\mathrm{CHCl}_{3}$ compared to buffer is due to the greater QY of the LY ( $\sim 2$-fold increase), overcompensating a $\sim 5 \%$ decrease in the overlap integral $J .^{16}$

The properties of LY are unmodified by conjugation to the polymer, such that the polymersomes offer a significant multiplexing advantage. However, of primary relevance to the proposed uses of photoswitchable probes are the attainable modulation of steady-state emission (quenching and dequenching) and the corresponding change in lifetimes. Experiments with the pure polymer PMA 9PC 75C12 2.5 LY in buffer solution provided disappointing initial results according to both measures, as seen in Table 1. 

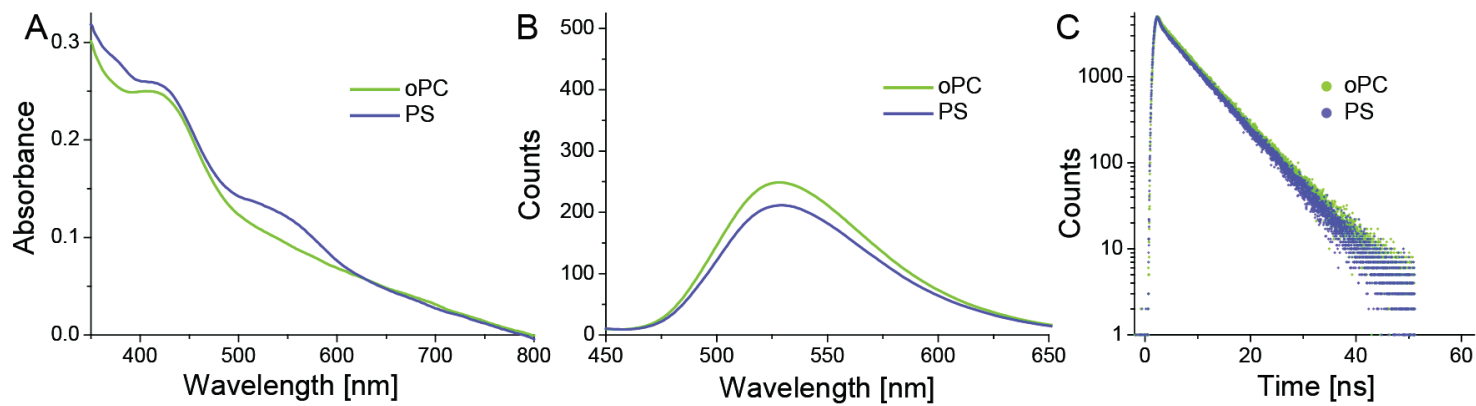

Figure 4: Spectra of Polymersomes. Irradiation: UV, $340 \pm 10 \mathrm{~nm}$ (irradiance $1.1 \mathrm{~mW} \mathrm{~cm}^{-2}$ ); A) absorbance B) steady-state fluorescence C) lifetime fluorescence.

Table 1: Properties of photomodulatable amphiphilic polymer in $\mathrm{CHCl}_{3}$ and SBB.

\begin{tabular}{lllllllll}
\hline \multicolumn{1}{c}{ Polymer } & $\begin{array}{l}\text { LY-cPC } \\
R_{\mathrm{o}}[\mathrm{nm}]\end{array}$ & $\begin{array}{l}\text { Emission } \\
\text { peak }[\mathrm{nm}]\end{array}$ & QY & $\begin{array}{l}\text { Quench } \\
{[\%]}\end{array}$ & $\begin{array}{l}\langle\tau\rangle_{\mathrm{amp}} \\
{[\mathrm{ns}]^{\mathrm{b}}}\end{array}$ & $\begin{array}{l}\text { Modulation } \\
\langle\tau\rangle_{\text {amp }}[\%]\end{array}$ & $\begin{array}{l}\mathrm{cPC} / \mathrm{LY} \text { in } \\
\text { PS state }\end{array}$ & $\alpha_{\mathrm{PS}}$ \\
\hline In CHCl3 & 4.4 & 502 & 0.47 & 60 & $8.0 \pm 0.3$ & 46 & $0.81 \pm 0.01$ & 0.22 \\
Polymersome & 3.9 & 529 & 0.25 & 20 & $1.6 \pm 0.2$ & 13 & $0.20 \pm 0.02$ & 0.06 \\
Mixed Polymer $^{\mathrm{a}}$ & 3.9 & 529 & 0.25 & 30 & $3.4 \pm 0.3$ & 21 & $0.55 \pm 0.04$ & 0.15 \\
\hline
\end{tabular}

[a] Polymersomes formed with PMA 9PC 75C12 2.5LY mixed in a 1:1 ratio with PMA 75C12 (a polymer which contains only dodecylamine chains). [b] Amplitude weighed mean fluorescence lifetime; excitation source, N-460 nanoLED.

The photomodulation decreased markedly from that observed in $\mathrm{CHCl}_{3}$. Experiments where a "dilution" of the composite polymer with a second polymer containing only dodecylamine chains (PMA 75C12) led to increases in all of the relevant properties: degree of quenching, $\mathrm{cPC} / \mathrm{LY}$ ratio, and $\langle\tau\rangle_{\text {amp }}$ (Mixed Polymer in Table 1). Previous experiments suggested that the inclusion of numerous voluminous dye groups in amphiphilic polymers negatively alters the efficiency of QD coating. ${ }^{11}$ In the case of polymersomes, increasing the number of large dye molecules relative to the dodecylamine chains probably leads to greater water permeability, diminishing the hydrophobic character of the interior. The photostationary states of PC in polymers placed in organic solvents and aqueous solutions are similar if the polymersome is well formed and compact $\left(\alpha_{\mathrm{PS}} \sim 0.22\right) .{ }^{17}$ However, $\alpha_{\mathrm{PS}}$ for the polymersomes generated in the present study was only 0.06 indicating that for PC molecules in a polar environment the extent of photoconversion induced by UV light diminishes greatly. In Figure 5 the observed $\mathrm{k}_{\mathrm{oc}}$ for $\mathrm{UV}$ photocyclization from oPC to $\mathrm{cPC}$ within the polymersome nanostructure was lower, $33 \pm 9 \mathrm{~s}^{-1}$, than of the single chain in organic solvent.
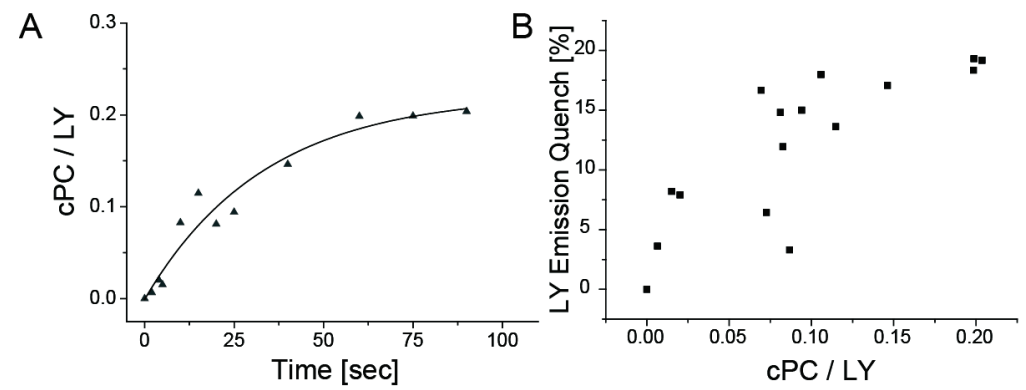

Figure 5: Photoconversion and quenching data (A) Graph of the evolution of cPC/LY as a function of UV (1.1 $\mathrm{mW} \mathrm{cm}{ }^{-2}$ ) irradiation time. Fit is monoexponential. (B) Quenching of LY fluorescence as a function of cPC/LY. 
The correlation between the LY quench and the amount of cPC could not be satisfactorily fit. The data was irregular, as can be seen in Figure 5B and no adequate model to represent it could be found. In conclusion, although the polymersomes comprised bright tags by virtue of incorporating many LY fluorophores, they were too large for many applications. Furthermore, the degree of photomodulation, $\leq 20 \%$ according to both steady state intensity and time correlated fluorescence, was marginal as compared to the other constructs.

\subsection{Dual-Color Photoswitchable Nanoparticles}

The final characterization of interest was the coating of the polymer onto NPs. The fact that the self-assembly of the polymer and QD is the result of hydrophobic interactions led to the expectation that the space between the QD surface and the polymer should provide an excellent microenvironment for PC switching. It was anticipated that a NP template would therefore enhance the properties exhibited by the polymersomes. Some of the most common organic surface ligands on QDs are trioctylphosphine oxide (TOPO), trioctylphosphine (TOP), and 1-hexadecylamine (HDA). All three ligands are present in the Series A CSS $635 \mathrm{~nm}$ QDs CdSe/CdS/ZnS core-shell-shell nanoparticles ${ }^{18}$ (CAN GmbH, Hamburg) which I utilized in these experiments.

The coating of the QD with the polymer is a self-assembly mechanism directed by the hydrophobic interactions of the alkyl chains of the polymer with the surface ligands of the QD. ${ }^{2}$ The carboxyl groups, which are formed during the nucleophilic attack on the anhydride groups, orient externally and provide the negative charge stabilizing the NP in buffer solution (50 mM SBB). The distribution of polymers on the QD surface was presumed to be random.

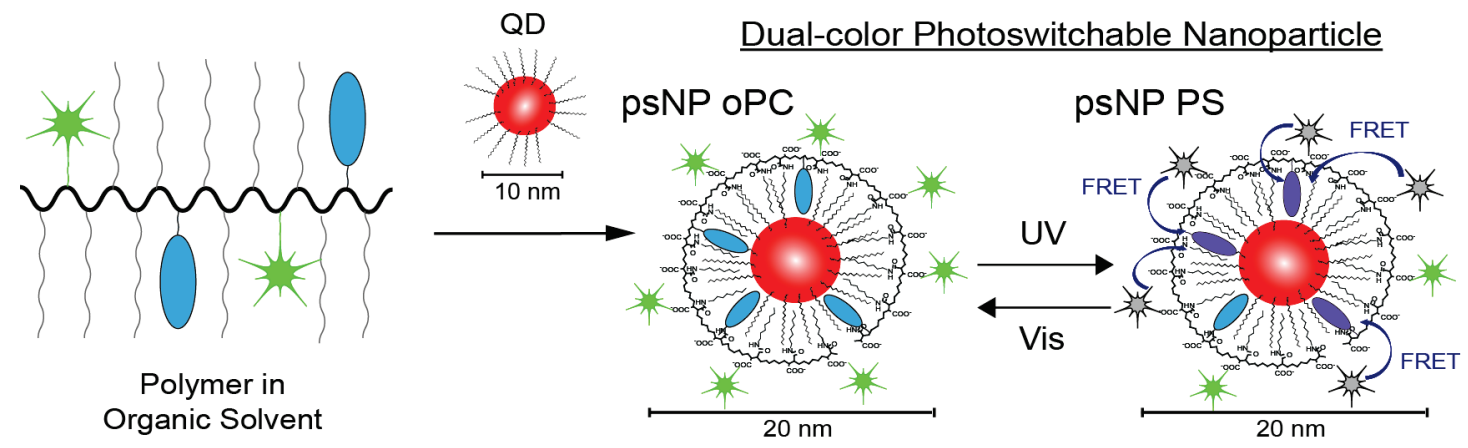

Scheme 5: Schematic representation of psNPs and their photomodulation.

By utilizing a QD with an emission at a longer wavelength $(635 \mathrm{~nm})$ the anticipated improvements in key parameters were achieved, and an imaging construct with the added advantage of a second emission band was created. The entire construct consisting of the photomodulatable polymer coating the red-emitting QD is referred to as a dual-color photoswitchable nanoparticle (psNP). The psNPs were stable for over a year, without demonstrable changes in properties, and exhibited resistance to $\mathrm{pH}$ changes in the range of 8-12 and a salt concentration lowered to $0.5 \mathrm{mM}$. 
These psNPs are suitable for ratiometric photomodulation, with the QD functioning as a very stable internal standard. In addition, the QDs can also be imaged independently by exciting at a wavelength beyond the spectral region of LY. Except for a reduced intensity the desirable properties of the QD emission are maintained.

\subsubsection{Physical Characterization of psNP}

The psNPs were imaged at the lab of Dr. Dietmar Riedel (Electron Microscopy, MPIbpc) using transmission electron microscopy (TEM). The measured diameter of the naked QD in organic solvent was $9.2 \pm 0.6 \mathrm{~nm}$ and $9.9 \pm 0.5 \mathrm{~nm}$ for the QDs coated with the polymer. Nanoparticle tracking measurements, for the determination of hydrodynamic radii, were able to observe the psNP, but they were below the quantification limit ( $>20 \mathrm{~nm}$ diameter). The QD fluorescence overlapped with the dynamic light scattering (DLS) laser and prevented its utilization to estimate a hydrodynamic radius. An indirect estimate was obtained from FRET (See Section 2.3.2) and an $\sim 18-19 \mathrm{~nm}$ diameter in solution (not the same as a hydrodynamic diameter) was calculated. This value is compatible with the reported ratio of 2 for the hydrodynamic diameter divided by the TEM diameter for polymer coated NPs. ${ }^{19}$ Size exclusion chromatography also yielded consistent results. Using an analytical Superdex200 (GE Healthcare) column the psNP eluted consistently after the empty polymersome fraction.

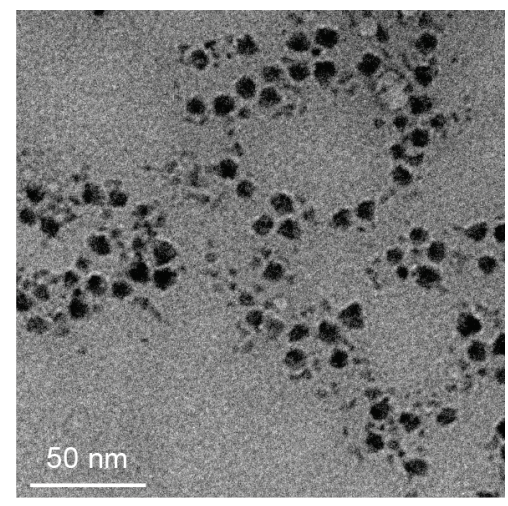

Figure 6: TEM image of psNP.

The spectral properties, discussed in Section 2.3.2, were consistent with a location of the PC groups in the hydrophobic space between the QD surface and the polymer, with the LY external to the polymer coat, i.e. exposed to the aqueous medium.

\subsubsection{Spectral Characterization of the LY and QD Emission Components of psNP}

The dual-color psNP was studied spectroscopically by absorbance, steady-state fluorescence, and TCSPC-monitored fluorescence decay.

Estimates were obtained from the absorbance spectra of the number of dyes and polymer chains coating each QD. The LY and PC concentrations were calculated in a manner similar to that that used in the characterization of the polymer. Assigning $\mathrm{Abs}_{625}$ in the oPC state entirely to the QD, led to a determination of the QD concentration and, thereby, the psNP composition. In the PS state, 
there were $52 \pm 2 \mathrm{LY}$ from an equivalent number of polymer chains, and $35 \pm 2 \mathrm{cPC}$ (out of a total of $\sim 190$ PC) per QD. The large number of LY groups is important when the imaging construct is considered. One reason has to do with the much larger absorption cross-section ( 150 -fold) of the QD itself. Even at $430 \mathrm{~nm}$, the LY absorption maximum, and with $\sim 50 \mathrm{LYs}$ per QD out of 4 absorbed photons 3 will be by the QD and 1 by the LY. A second consideration is the capacity of the QD to act as a FRET acceptor for a LY donor. In view of the very favorable $\mathrm{R}_{\mathrm{o}}$ of $6.9 \mathrm{~nm}$, FRET decreases the LY emission significantly. Therefore to have approximately equivalent emission it is important that many LY groups be present.

Assuming a homogenous PC population the value of $\alpha_{P S}$ on psNPs was $0.17-0.19$, much higher than the 0.06 observed with polymersomes and similar to 0.22 in $\mathrm{CHCl}_{3}$. The fact that both the LY and the QD acted as FRET donors to the cPC acceptor led to a globally more efficient cycloreversion of the $\mathrm{cPC}$ to the oPC form. The high $\alpha_{\mathrm{PS}}$ in the assembled psNP proved that the PC groups were located predominantly in the hydrophobic compartment defined by the QD surface and the overlying polymer cap.

The emission maximum and QY point to LY being exposed to the aqueous medium in both the psNP and the polymersomes. A certain number of groups may have been situated in the more hydrophobic microcompartment of the psNP, giving rise to the slight hypsochromic shift $(\sim 4 \mathrm{~nm})$ in the emission peak as well as the increase in mean lifetime. ${ }^{15}$ The reduced QY probably reflected FRET from the $\mathrm{LY}$ to the $\mathrm{QD}$, as discussed earlier. Using this assumption a mean $\mathrm{r}_{\mathrm{DA}}(\mathrm{LY} \rightarrow \mathrm{QD})$ of $9.2 \mathrm{~nm}$ was computed. Assuming that the LY are on the outer layer of the psNP, it follows that the diameter of psNP in solution was $18-19 \mathrm{~nm}$.

The primary observation in these studies was the large increase in both steady-state quenching and lifetime modulation of the LY on the psNP, which was attributed to two main factors, the first being the larger $\alpha_{\mathrm{PS}}$ obtained by placing the PC in the hydrophobic microenvironment. The almost 3fold greater number of $\mathrm{cPC}$ per LY implies an equivalent increase in the acceptor cross-section. ${ }^{16} \mathrm{~A}$ second factor is the reduced size of the final NP, with the QD acting as a more efficient template for concentrating the polymer. The reduced surface area and higher acceptor surface density lead to more efficient intermolecular FRET between neighboring polymers. This fact is further explored in section 2.3.4. 

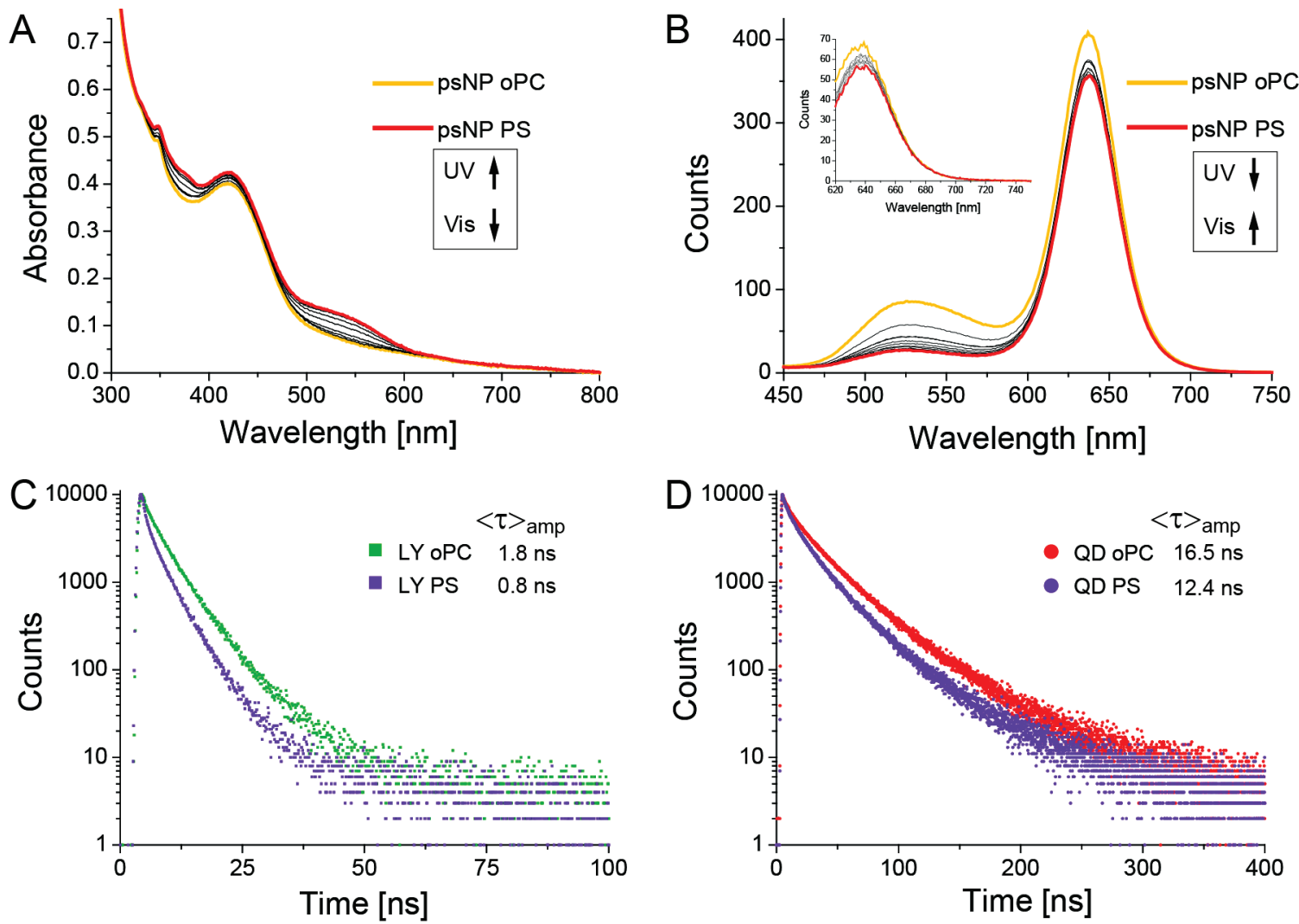

Figure 6: Spectroscopic monitoring of dual-color psNP. Irradiation was: UV, $340 \pm 10 \mathrm{~nm}$ (irradiance $1.1 \mathrm{~mW}$ $\left.\mathrm{cm}^{-2}\right)$; Vis, $545 \pm 10 \mathrm{~nm}\left(6.2 \mathrm{~mW} \mathrm{~cm}^{-2}\right)$. Temperature, $20{ }^{\circ} \mathrm{C}$. (A) Absorbance spectra. (B) Fluorescence spectra, excitation at $420 \mathrm{~nm}$. Inset: Fluorescence spectra, excitation at $600 \mathrm{~nm}$. (C,D) Time-resolved fluorescence decays. Excitation source, N-460 nanoLED; emission monitored at either $530 \mathrm{~nm}$ (C) or $635 \mathrm{~nm}$ (D).

The properties of the QD component of the psNP are summarized in the lower half of Table 2, in which a comparison is made with QDs coated with a polymer containing only dodecylamine chains, i.e. lacking PC. The reduction in QY and lifetime were attributed to a lower coating efficiency of the QD with the polymer containing voluminous dye groups. ${ }^{11}$ QDs lacking such moieties exhibit a greater QY, comparable to that in organic solvents such as $\mathrm{CHCl}_{3}$.

Table 2: Properties of LY and QD emitting species in different preparations. ${ }^{\mathrm{a}}$

\begin{tabular}{|c|c|c|c|c|c|c|c|}
\hline LY & $\begin{array}{l}\text { Emission } \\
\text { wavelength [nm] }\end{array}$ & QY & $\begin{array}{l}\text { Quench } \\
{[\%]}\end{array}$ & $\langle\tau\rangle_{\mathrm{amp}}[\mathrm{ns}]^{\mathrm{c}}$ & $\begin{array}{l}\text { Modulation } \\
\langle\tau\rangle_{\mathrm{amp}}[\%]\end{array}$ & $\begin{array}{l}\mathrm{cPC} / \mathrm{LY} \text { in } \mathrm{PS} \\
\text { state }\end{array}$ & $\alpha_{\mathrm{PS}}$ \\
\hline psNP & 525 & 0.21 & 69 & $1.8 \pm 0.3$ & 56 & $0.67 \pm 0.04$ & 0.19 \\
\hline Polymersome & 529 & 0.25 & 20 & $1.6 \pm 0.2$ & 13 & $0.20 \pm 0.02$ & 0.06 \\
\hline QD & & & & & & $\begin{array}{l}\mathrm{cPC} / \mathrm{QD} \\
\text { in PS state }\end{array}$ & \\
\hline psNP & 640 & 0.08 & 12 & $16.5 \pm 0.2$ & 25 & $35 \pm 2$ & 0.19 \\
\hline PMA-QD ${ }^{b}$ & 640 & 0.21 & - & $20.8 \pm 0.7$ & - & - & - \\
\hline
\end{tabular}

[a] All samples measured in SBB. Excitation for steady-state fluorescence at $420 \mathrm{~nm}$. [b] CdSe/CdS/ZnS $635 \mathrm{~nm}$ QDs coated with PMA 75C12 (a polymer which contains only dodecylamine chains). [c] Amplitude weighed mean fluorescence lifetime. Excitation source, N-460 nanoLED; detection at $525 \mathrm{~nm}$ for LY and $635 \mathrm{~nm}$ for QD. 
A small but unexpected change was observed in the QD fluorescence (12\%) upon UV irradiation of the psNP. In the case of using an excitation wavelength at which the LY absorbance was considerable, the QD quenching was as high as $14 \%$. The $\mathrm{R}_{\mathrm{o}}$ for a single QD-cPC donor-acceptor pair is only $2.3 \mathrm{~nm}$, such that little discernible FRET was expected. Yet due to the 35 acceptors present in the PS state the effective $\mathrm{R}_{\mathrm{o}}$ would be $4.2 \mathrm{~nm}$, from which an $\mathrm{r}_{\mathrm{DA}}$ of $\sim 6 \mathrm{~nm}$ was estimated, assuming that the QD sensed all acceptors as equivalent.

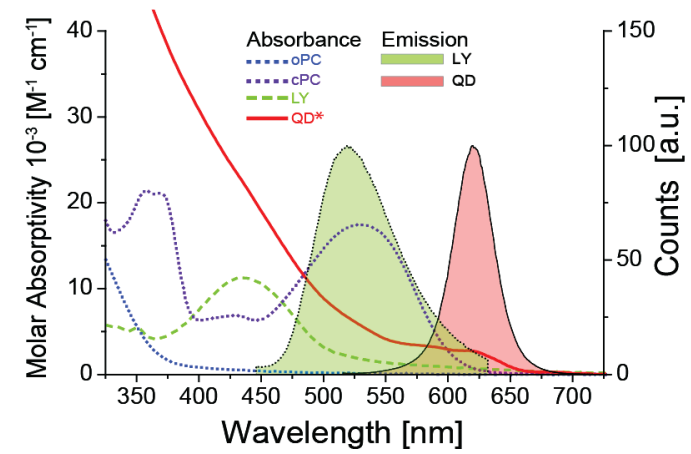

Figure 7: Superposition of absorbance (solid lines) and emission (filled areas) spectra of all the components of the psNP. The emission spectra are normalized by their peak values. *Molar absorptivity of the QD is divided by 100 to fit on the same scale.

Unexpectedly, the relative decrease in fluorescence lifetime, $\langle\tau\rangle_{\mathrm{amp}}$ was double that of steadystate fluorescence ( $25 \%$ vs. $12 \%$ ). In the case of the QD in the oPC state I presume that the complex energy transfer system extends the excited state such that mean acceptor lifetime increases by a factor proportional to the transfer efficiency and the donor (LY)/acceptor (QD) ratio. Scheme 6 presents a schematic view of the proposed mechanism, the basis of which can be stated as follows. Excitation in the lifetime measurements was at $460 \mathrm{~nm}$, a wavelength at which both fluorophores absorb. LY is capable of acting as a FRET donor to the QD. When the detection is centered at $635 \mathrm{~nm}$, both the direct and sensitized emissions of the QD are registered. However, the lifetime component(s) of the sensitized emission will be longer than those due to direct excitation of the $\mathrm{QD}^{20}$ and can be regarded as an initial offset for the sensitized emission pathway. When the psNP is in the PS state the energy transfer from the LY to the QD decreases considerably due to the competing pathway presented by the cPC acceptor (see Scheme 6, specifically $\mathrm{k}_{\mathrm{t} 2}$ ). In addition to the classical decrease in lifetime due to the QD-cPC FRET the QD no longer exhibits the extended lifetime components due to the abatement of the sensitized emission experimented in the oPC state. 


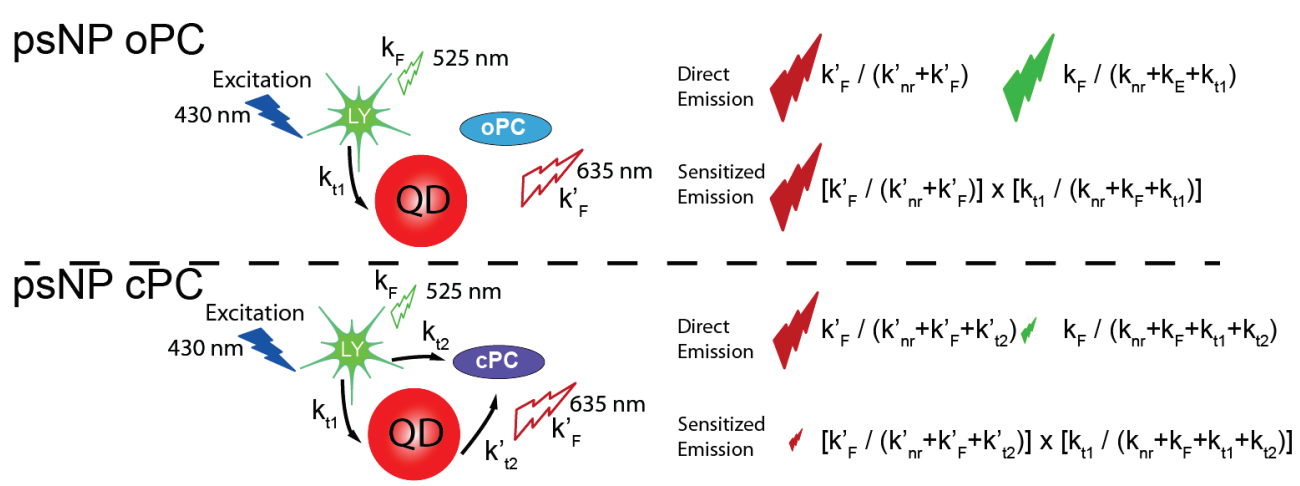

Scheme 6: Schematic representation of the energy transfer system. LY is denoted as k and QD is denoted as $\mathrm{k}^{\prime}$. $\mathrm{k}_{\mathrm{F}}$, emission; $\mathrm{k}_{\mathrm{nr}}$, nonradiative pathways; $\mathrm{k}_{\mathrm{t} 1}$, LY to QD energy transfer; $\mathrm{k}_{\mathrm{t} 2}$, fluorophore to cPC energy transfer. Right side, arrows denoting emission intensities normalized to $100 \%$ in the oPC form.

The hypothesis is supported by the observed fits to the decay curves of the QD in the psNP. Due to the complex core/shell/shell+polymer shell composition of the psNP, 4 lifetimes (more correctly eigenvalues) were required to accurately fit the data and to determine $\langle\tau\rangle_{\mathrm{amp}}$. As is seen in Figure 8 the shorter lifetimes $\tau_{1,2}$ were the same for both states, yet the longer lifetimes $\tau_{3,4}$ decreased by $\sim 20 \%$ in the PS state. These longer decay components are the most influenced by sensitized emission pathways. The amplitudes of the individual lifetimes in the two states did not show significant differences.
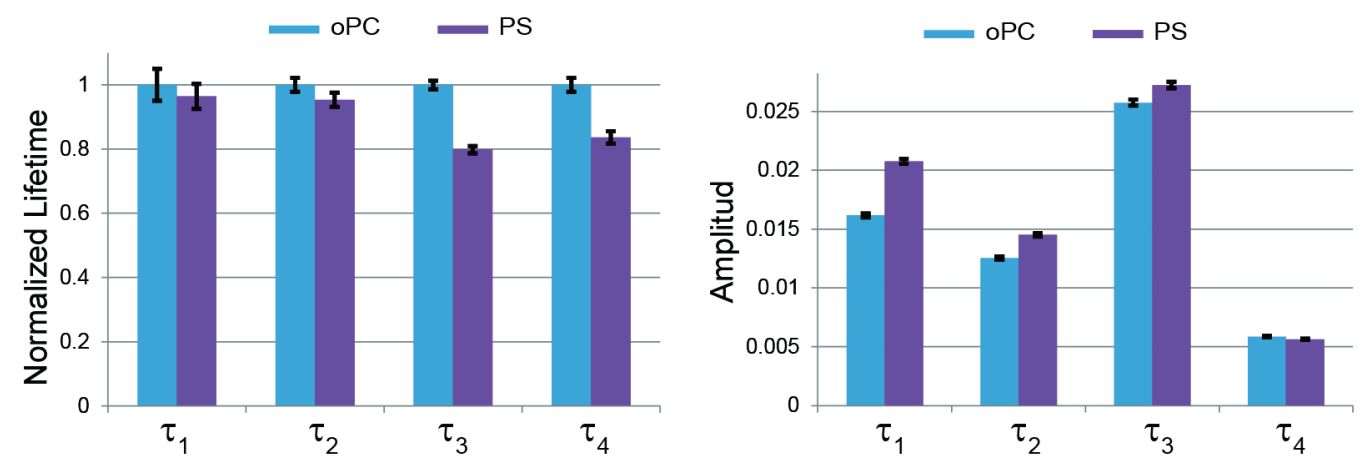

Figure 8: A) Individual decay constants of QD emission from psNPs normalized by the values for the oPC state. B) Individual amplitude of corresponding decay constants of QD emission from psNPs.

\subsection{3 psNP as Ratiometric Probes}

The modulations of both fluorophores in psNP does not interfere with its function as a ratiometric imaging particle. Although both emissions decrease in the PS state, the relative changes are quite different. An Emission Ratio (ER) was defined as the QD emission at $635 \mathrm{~nm}$ divided by the LY emission at $525 \mathrm{~nm}$. Upon excitation at $420 \mathrm{~nm}$, there was up to a 3-fold change in the ER (4.3 to 13.2). Figure 9 shows various cycles of psNP through the oPC and PS states generated by alternating UV (lower values) and visible (higher values) irradiation. Progressive photobleaching of the LY was detected as the sample was irradiated, reaching 15\% after 10 Vis-UV cycles. QDs are more photostable than organic dyes and in fact often photobrighten upon cyclical irradiation. ${ }^{21}$ The LY photobleaching is not a limitation for ratiometric imaging, as can be observed in Figure 9B. The ER 
change decreased slowly as the psNP was irradiated but the cycle-to-cycle decrease was less than the uncertainty in the measurements. The mean ER increase was $120 \pm 9 \%$ with only a $6 \%$ decrease due to photobleaching over the course of the 10 cycles.
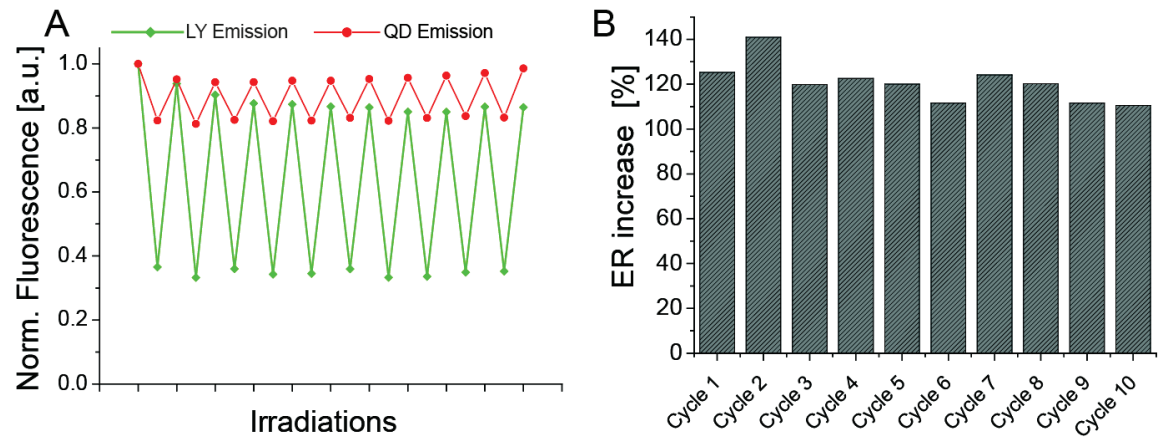

Figure 9: Spectroscopic monitoring of psNP cycled through oPC-PS states. Irradiation was for $150 \mathrm{~s} ; 340 \pm 10$ $\mathrm{nm}\left(1.1 \mathrm{~mW} \mathrm{~cm}^{-2}\right)$ and $545 \pm 10 \mathrm{~nm}\left(6.2 \mathrm{~mW} \mathrm{~cm}^{-2}\right)$. Temperature, $20^{\circ} \mathrm{C}$. (A) Emission of QD $(635 \mathrm{~nm})$ and LY $(525 \mathrm{~nm})$ after each irradiation, with excitation at $420 \mathrm{~nm}$. (B) Increase in the ER during each cycle upon transition from the $\mathrm{oPC}$ to the $\mathrm{cPC}$ state.

\subsubsection{Spectral Kinetics and FRET Systems}

Similar to what was realized for the polymer and polymersome system, intermediate states provide additional information about the psNP. Such intermediate states were generated by applying pulses of UV irradiation to a sample in the open form, generating populations differing in cPC content and quenching of LY.

The conversion from oPC to cPC induced by exposure to UV light was fit with an exponential function. The apparent first order rate constant $\mathrm{k}_{\mathrm{oc}}^{\prime}$ was $52 \pm 9 \mathrm{~s}^{-1}$, a similar value to that obtained for the polymer in $\mathrm{CHCl}_{3}\left(58 \pm 2 \mathrm{~s}^{-1}\right)$.

The data for the quenching of LY fluorescence as a function of the $\mathrm{CPC} / \mathrm{LY}$ ratio was analyzed in two ways. First, I present the data as analyzed by the simplest FRET equation and then an expression applicable to the specific physical model of the psNP developed by Dr. Thomas Jovin. ${ }^{22}$ Both fits resulted in the same curve as observed in Figure 10B, it is the interpretation of the curve that varied. The fits were not improved by adding static quenching, therefore both models assumed the quenching to be due entirely to FRET. In the psNP the cPC was sequestered in a different microenvironment than LY with little or no possibility for appreciable $\pi-\pi$ static quenching.

The FRET efficiency equation (Equation 2 Introduction) can be rewritten as a function of the number of acceptors as follows

$$
E=x \gamma /(1+x \gamma)
$$

Where $\gamma$ is the same as in equation 6 and $\mathrm{x}=\mathrm{cPC} / \mathrm{LY}$. Using Equation 8 to fit the data, a value of $\gamma=2.4 \pm 0.1$ is obtained; if an $R_{o}=3.9 \mathrm{~nm}$ (Table 2) is utilized an $r_{D A}$ of $3.4 \pm 0.2 \mathrm{~nm}$ is the final 
result. This model does not take into consideration the physical distribution of the dyes, yet serves as a general estimation.
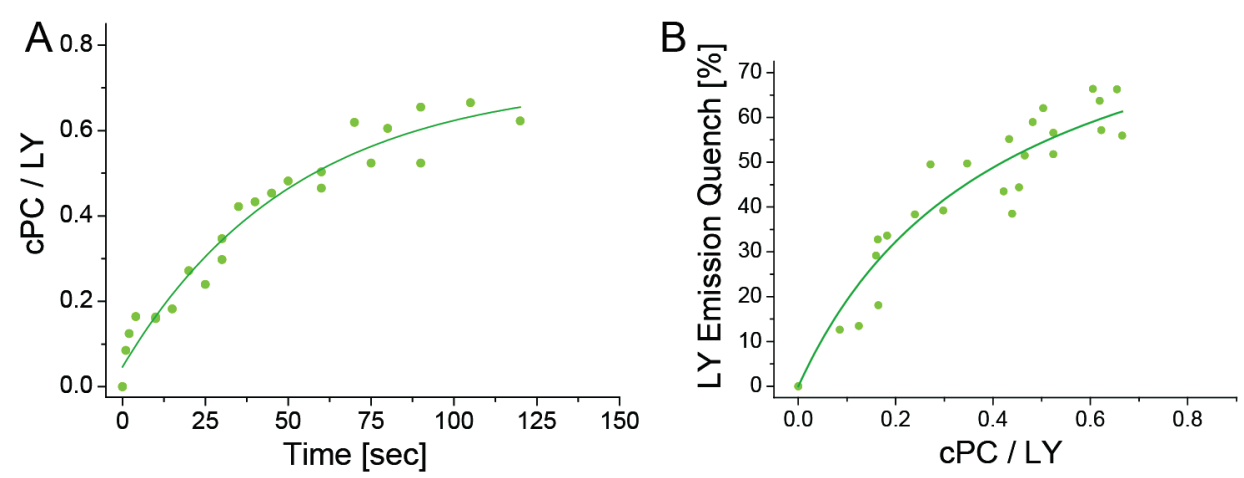

Figure 10: Photoconversion and quenching data (A) Graph of the evolution of cPC/LY as a function of UV (1.1 $\mathrm{mW} \mathrm{cm}{ }^{-2}$ ) irradiation time. Fit is monoexponential. (B) Quenching of LY fluorescence as a function of cPC/LY. Fit Corresponds to both Equation 8 and 9.

The comprehensive treatment of the psNP quenching by $\mathrm{cPC}$ was based on the assumption that the polymers are distributed densely on the surface of the nanoparticle such that intermolecular transfer between LY donors and cPC acceptors conjugated to different polymer chains will take place (Scheme 7). The psNP configuration is represented as dual concentric shells of acceptor cPC moieties (inner shell, radius $r_{A}$ ) and donor LY (outer shell, radius $r_{D}=r_{A}+\Delta r$ ). The center of the dye shells is the center of the QD. Therefore I can use the previously estimated values of $r_{A}(6 n m)$ or $r_{D}(9.2 n m)$ from section 2.3.2. Integration of the incremental transfer rate over the entire spherical surface yields the following expression for the FRET efficiency, given as a quenching percentage:

$$
\text { Quench } \%=100 \frac{a x}{1+a x} ; a=p\left(\frac{\delta^{2}+2 \delta+2}{\delta^{4}(\delta+2)^{4}}\right) \mu \quad \text { Eq. [9] }
$$

where $\mu=\left(\mathrm{R}_{0} / \mathrm{r}_{\mathrm{A}}\right)^{6} ; \delta=\Delta \mathrm{r} / \mathrm{r}_{\mathrm{A}} ; p=\mathrm{LY} / \mathrm{psNP}=52 \pm 2$; and $\mathrm{x}=\mathrm{cPC} / \mathrm{LY}$. The fit of Eq. 9 to the psNP data yielded $\mathrm{a}=2.44 \pm 0.12$ and a corresponding set of possible combinations of $\mu$ and $\delta$. For $\mathrm{R}_{\mathrm{o}}=3.9$ $\mathrm{nm}$ and $\mathrm{r}_{\mathrm{A}}=6.0 \mathrm{~nm}, \delta=0.58-0.61$ from which an $\mathrm{r}_{\mathrm{D}}$ of 9.5-9.7 nm and $\Delta \mathrm{r}$ of 3.5-3.7 are obtained. These values are robust and consistent with the other estimates of these values.

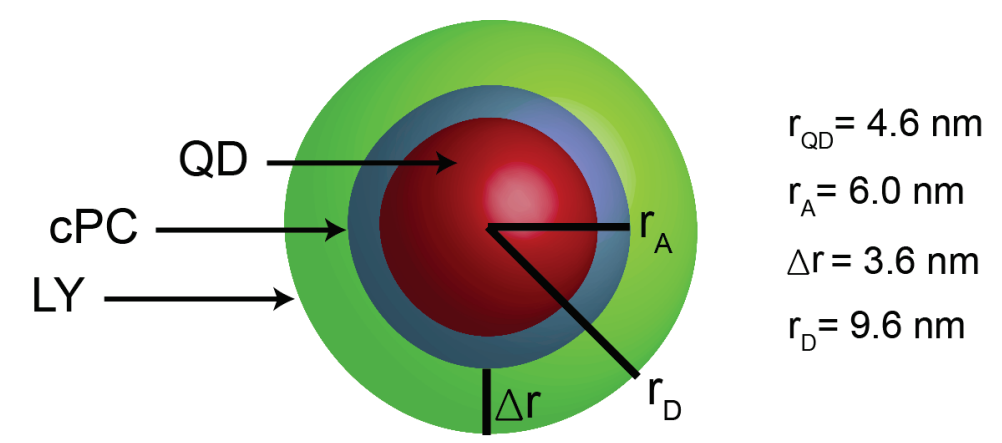

Scheme 7: Schematic representation of the FRET model from Eq. 9. 


\subsection{Comparisons and Interpretation}

The photoconversion equilibria $\left(\alpha_{\mathrm{PS}}\right)$, which is directly related to the $\mathrm{cPC} / \mathrm{LY}$ value determined at the PS state, in the presented systems (polymer in $\mathrm{CHCl}_{3}$, polymersomes, and psNP) is shifted in response to the respective donor-acceptor configurations and microenvironments of the PC. The oPC $\rightarrow$ cPC conversions can be observed in Figure 11, where the curves are fit to monoexponential functions, and the resulting values presented in Table 3.
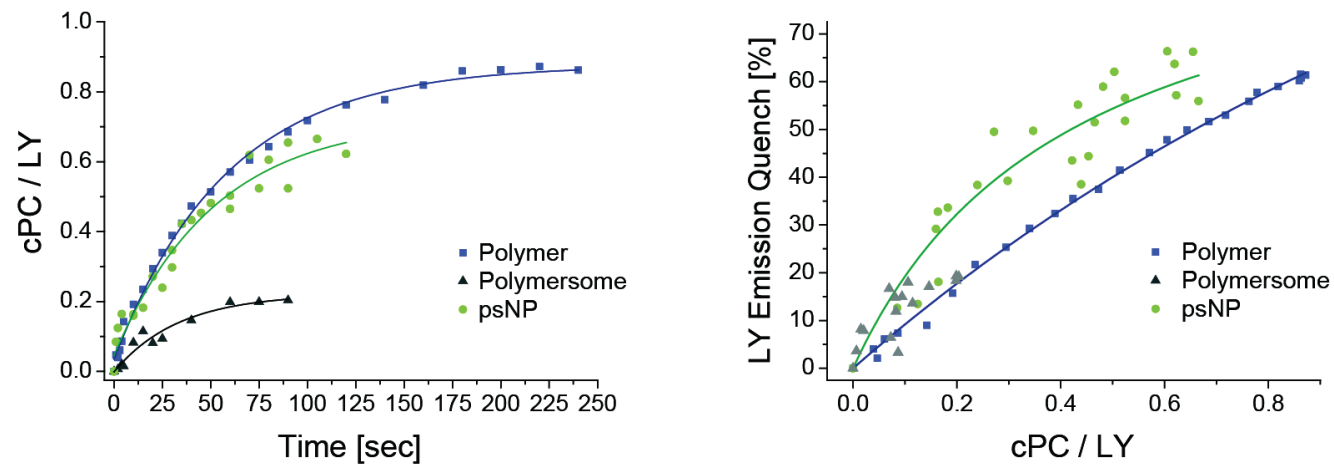

Figure 11. Photoconversion and quenching data (A) Graph of the evolution of cPC/LY as a function of UV (1.1 $\mathrm{mW} \mathrm{cm}{ }^{-2}$ ) irradiation time. Fits are monoexponential. (B) Quenching of LY fluorescence as a function of cPC/LY.

Table 3: Values of photoconversion and quenching

\begin{tabular}{llll}
\hline & Polymer in $\mathrm{CHCl}_{3}$ & Polymersome & psNP \\
\hline $\mathrm{k}_{\mathrm{oc}}^{\prime}\left[\mathrm{s}^{-1}\right]$ & $58 \pm 2$ & $52 \pm 9$ & $33 \pm 9$ \\
$\mathrm{r}_{\mathrm{DA}}[\mathrm{nm}]$ & $2.6-3.1^{\mathrm{a}}$ & $-{ }^{\mathrm{b}}$ & $3.5-3.7^{\mathrm{c}}$ \\
\hline
\end{tabular}

[a] From Eq. 5 [b] An adequate model was not found to represent the polymersome data. [c] From Eq. 9, $\Delta r \sim$ rDA.

The local environment sensed by the PC between the QD surface and the polymer cap on a well-formed psNP is similar to that in $\mathrm{CHCl}_{3}$, whereas the polymersomes experience a very different medium. It is important to note that the apparent rate constants also incorporate a contribution representing the cycloreversion of cPC to oPC induced by FRET from either the LY, or the LY and QD in the case of the psNP. ${ }^{23}$

If the individual properties of the polymer in $\mathrm{CHCl}_{3}$ to those in psNP are compared it can be seen that in $\mathrm{CHCl}_{3}$ the $\mathrm{LY}$ has a higher QY, the value of $\mathrm{cPC} / \mathrm{LY}$ is greater in the PS state, and even an estimate of the $r_{D A}$ is smaller. All of this should lead to more efficient energy transfer, ${ }^{16}$ yet the quenching of the LY is higher on the psNP. From the quantitative considerations it can be seen that the facile quenching of psNP, even at low cPC values, compared to the isolated polymer or polymersomes is attributable to the increased density and geometric dimensionality of the acceptor population (2D instead of 1D). This results in a greater number of potential acceptors per donor and thus the absence of a finite subpopulation devoid of acceptor. 


\subsection{Conclusions}

The step-by-step analysis of photoswitchable polymers has demonstrated the advantage of placing hydrophobic molecules between the QD and the polymer layer. In particular the PC molecules can efficiently photoconvert in both directions without fatigue and are placed near to the QD surface. In conclusion a nanoparticle template can drastically improve the properties of polymer micelles, creating a new class of psNP that demonstrate many properties of interest as probes in microscopy. The psNP has dual-color emission (525 and $635 \mathrm{~nm}$ ) with excitation at a single wavelength, small size ( $<20 \mathrm{~nm}$ diameter), large modulation of the LY emission both by steady state and time-resolved fluorescence ( $70 \%$ and 55\%, respectively), and a mean emission ratio (ER) change of $120 \%$. Further improvements can be considered, for example selecting a QD with an emission shifted even further to the red, thus eliminating the overlap of its emission with the PC absorbance. This should increase both the $\alpha_{P S}$ and the ER change of the psNP. A new optimized polymer could be synthesized with a slightly higher PC content and a fluorescent probe with greater photostability and brilliance.

\section{References}

1. Pellegrino, T.; Manna, L.; Kudera, S.; Liedl, T.; Koktysh, D.; Rogach, A. L.; Keller, S.; Rädler, J.; Natile, G.; Parak, W. J. Hydrophobic Nanocrystals Coated with an Amphiphilic Polymer Shell: A General Route to Water Soluble Nanocrystals. Nano Lett. 2004, 4, 703-707.

2. Sperling, R. A.; Parak, W. J. Surface Modification, Functionalization and Bioconjugation of Colloidal Inorganic Nanoparticles. Philos. Trans. R. Soc. A 2010, 368, 1333-1383.

3. Giordano, L.; Jovin, T. M.; Irie, M.; Jares-Erijman, E. A. Diheteroarylethenes as Thermally Stable Photoswitchable Acceptors in Photochromic Fluorescence Resonance Energy Transfer (pcFRET). $J$. Am. Chem. Soc. 2002, 124, 7481-7489.

4. Tong, R.; Hemmati, H. D.; Langer, R.; Kohane, D. S. Photoswitchable Nanoparticles for Triggered Tissue Penetration and Drug Delivery. J. Am. Chem. Soc. 2012, 134, 8848-8855.

5. Zhou, K.; Liu, H.; Zhang, S.; Huang, X.; Wang, Y.; Huang, G.; Sumer, B. D.; Gao, J. Multicolored PhTunable and Activatable Fluorescence Nanoplatform Responsive to Physiologic pH Stimuli. J. Am. Chem. Soc. 2012, 134, 7803-7811.

6. Murakami, M.; Miyasaka, H.; Okada, T.; Kobatake, S.; Irie, M. Dynamics and Mechanisms of the Multiphoton Gated Photochromic Reaction of Diarylethene Derivatives. J. Am. Chem. Soc. 2004, 126, 14764-14772.

7. Kwon, D.-H.; Shin, H.-W.; Kim, E.; Boo, D. W.; Kim, Y.-R. Photochromism of Diarylethene Derivatives in Rigid Polymer Matrix: Structural Dependence, Matrix Effect, and Kinetics. Chem. Phys. Lett. 2000, 328, 234-243.

8. Belfiore, L. A., Gaussian Statistics of Linear Chain Molecules and Crosslinked Elastomers. In Physical Properties of Macromolecules, John Wiley \& Sons, Inc.: 2010; pp 547-607.

9. Schroder, G. F.; Alexiev, U.; Grubmuller, H. Simulation of Fluorescence Anisotropy Experiments: Probing Protein Dynamics. Biophys J 2005, 89, 3757-3770. 
10. Gradinaru, C. C.; Marushchak, D. O.; Samim, M.; Krull, U. J. Fluorescence Anisotropy: From Single Molecules to Live Cells. Analyst 2010, 135, 452-459.

11. Díaz, S. A.; Giordano, L.; Jovin, T. M.; Jares-Erijman, E. A. Modulation of a Photoswitchable DualColor Quantum Dot Containing a Photochromic FRET Acceptor and an Internal Standard. Nano Lett. 2012, 12, 3537-3544.

12. Sillen, A.; Engelborghs, Y. The Correct Use of "Average" Fluorescence Parameters. Photochem Photobiol 1998, 67, 475-486.

13. Furstenberg, A.; Vauthey, E. Excited-State Dynamics of the Fluorescent Probe Lucifer Yellow in Liquid Solutions and in Heterogeneous Media. Photochem Photobiol Sci 2005, 4, 260-267.

14. Fernandez-Arguelles, M. T.; Yakovlev, A.; Sperling, R. A.; Luccardini, C.; Gaillard, S.; Medel, A. S.; Mallet, J. M.; Brochon, J. C.; Feltz, A.; Oheim, M.; Parak, W. J. Synthesis and Characterization of Polymer-Coated Quantum Dots with Integrated Acceptor Dyes as FRET-Based Nanoprobes. Nano Lett 2007, 7, 2613-2617.

15. Mishra, P. P.; Koner, A. L.; Datta, A. Interaction of Lucifer Yellow with Cetyltrimethyl Ammonium Bromide Micelles and the Consequent Suppression of Its Non-Radiative Processes. Chem. Phys. Lett. 2004, 400, 128-132.

16. Jares-Erijman, E. A.; Jovin, T. M. FRET Imaging. Nat. Biotechnol. 2003, 21, 1387-1395.

17. Díaz, S. A.; Menendez, G. O.; Etchehon, M. H.; Giordano, L.; Jovin, T. M.; Jares-Erijman, E. A. Photoswitchable Water-Soluble Quantum Dots: pcFRET Based on Amphiphilic Photochromic Polymer Coating. ACS Nano 2011, 5, 2795-2805.

18. Talapin, D. V.; Mekis, I.; Götzinger, S.; Kornowski, A.; Benson, O.; Weller, H. CdSe/CdS/ZnS and CdSe/ZnSe/ZnS Core-Shell-Shell Nanocrystals. J. Phys. Chem. B 2004, 108, 18826-18831.

19. Sperling, R. A.; Liedl, T.; Duhr, S.; Kudera, S.; Zanella, M.; Lin, C. A. J.; Chang, W. H.; Braun, D.; Parak, W. J. Size Determination of (Bio)Conjugated Water-Soluble Colloidal Nanoparticles: A Comparison of Different Techniques. J. Phys. Chem. C 2007, 111, 11552-11559.

20. Xu, H.; Huang, X.; Zhang, W.; Chen, G.; Zhu, W.; Zhong, X. Quantum Dots Acting as Energy Acceptors with Organic Dyes as Donors in Solution. ChemPhysChem 2010, 11, 3167-3171.

21. Carrillo-Carrion, C.; Cardenas, S.; Simonet, B. M.; Valcarcel, M. Quantum Dots Luminescence Enhancement Due to Illumination with UV/Vis Light. Chem. Commun. 2009, 5214-5226.

22. Diaz, S. A.; Giordano, L.; Azcarate, J. C.; Jovin, T. M.; Jares-Erijman, E. A. Quantum Dots as Templates for Self-Assembly of Photoswitchable Polymers: Small, Dual-Color Nanoparticles Capable of Facile Photomodulation. J. Am. Chem. Soc. 2013, 135, 3208-3217.

23. Métivier, R.; Badré, S.; Méallet-Renault, R.; Yu, P.; Pansu, R. B.; Nakatani, K. Fluorescence Photoswitching in Polymer Matrix- Mutual Influence between Photochromic and Fluorescent Molecules by Energy Transfer Processes. J. Phys. Chem. C 2009, 113, 11916-11926. 


\section{CHAPTER 3: PHOTOSWITCHABLE QUANTUM DOTS}

\section{Introduction}

Of the fluorescent probes currently available for biological imaging, many exploit the unique properties of QDs: broad excitation, narrow emission, photostability, and brightness. QDs are replacing traditional organic fluorophores in many areas, including multiplexed analysis, cell sorting and tracking, as well as cellular and molecular imaging. ${ }^{1}$ QDs can also function as FRET donors and acceptors as was seen in Chapter 2. In the previous chapter the fluorescence emission of the QD donor was modulated by manipulating the state of the PC acceptor with cycles of UV-visible irradiation. A QD with a greater FRET overlap integral $(J)$, one that emits around $550 \mathrm{~nm}$, would present increased modulation, for which immediate applications come to mind in both cellular tracking, e.g. FRAP and FLIM, and in super-resolution fluorescence localization microscopy. ${ }^{2,3}$ In addition, techniques such as STORM or SOFI can utilize controllable and stable transitions between fluorophore bright and dark states for sub-diffraction detection. ${ }^{4,5}$
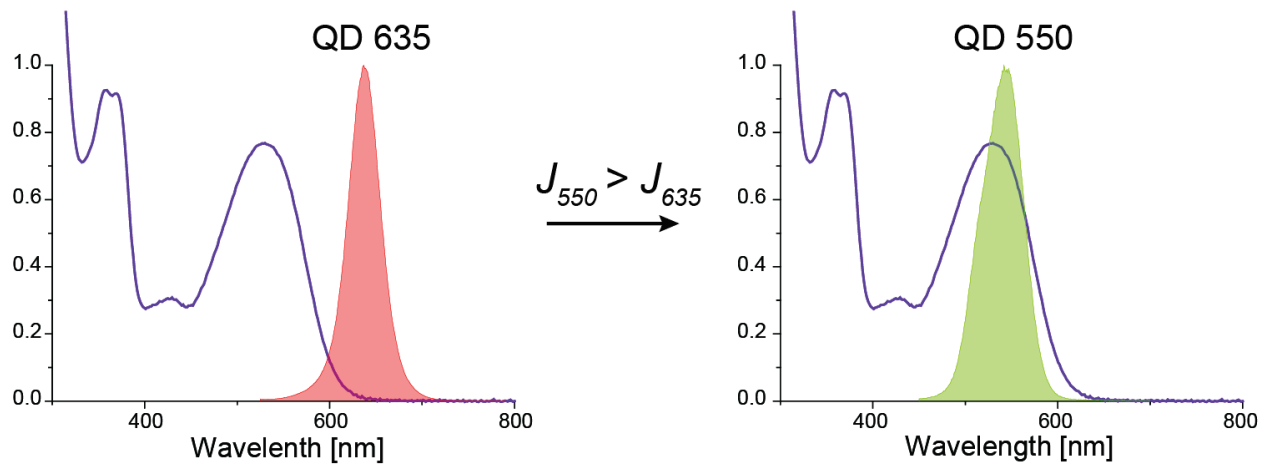

Figure 1: Increase in $J$ overlap integral by using a $550 \mathrm{~nm}$ QD. The purple line represents $\mathrm{cPC}$ absorbance and the areas are QD emission.

In the field of biocompatible sensors the development of new organic dyes and various photoswitchable fluorescent proteins has represented a tremendous boon to cellular biologists. ${ }^{6}$ Nonetheless the brightness and photostability of available fluorescent proteins and most dyes remains inferior to those of QDs. Previous publications have demonstrated pcFRET applied to QDs as well as organic donors. ${ }^{7,8}$ Yet the greatly reduced efficiency and stability of photocycling in aqueous media ${ }^{9}$ limited previous QD pcFRET examples to use in organic solvents. ${ }^{10,11}$ Within the framework of this thesis a photochromic acceptor was conjugated to amphiphilic polymers which efficiently coated QDs creating a hydrophilic and stable photoswitchable QD. I reiterate the considerations presented in Chapter 2. This architecture permits the exploitation of the hydrophobic microenvironment between the surface of the semiconductor QD and the external surface of the assembled NP. By placing the FRET acceptors in this compartment two goals are achieved: providing a chemical environment conducive to efficient and stable photoswitching and placement of the PC groups in a restricted zone, at a short distance to the QD surface. 
Initial thought was given to ligand exchange strategies, but capping strategies better preserve the stability and quantum yield of the QD in comparison to ligand exchange and was deemed superior. ${ }^{12}$ The synthetic procedures for capping strategies are also simpler.

The chosen structural design provides an effective pcFRET probe in a small ( $\sim \mathrm{nm}$ diameter) water soluble package. Fatigue, due to the thermal conversion of the acceptor and/or photobleaching of the donor, is minimized while retaining the superior properties of the original QD. Polymer coated QDs are also competent for cellular uptake. ${ }^{13}$ Throughout the following discussion, the construct comprising the $550 \mathrm{~nm}$ emitting QD core, the organic ligands attached to the QD, and the amphiphilic PC polymer coating, will be referred to as a photoswitchable QD (psQD). ${ }^{14}$

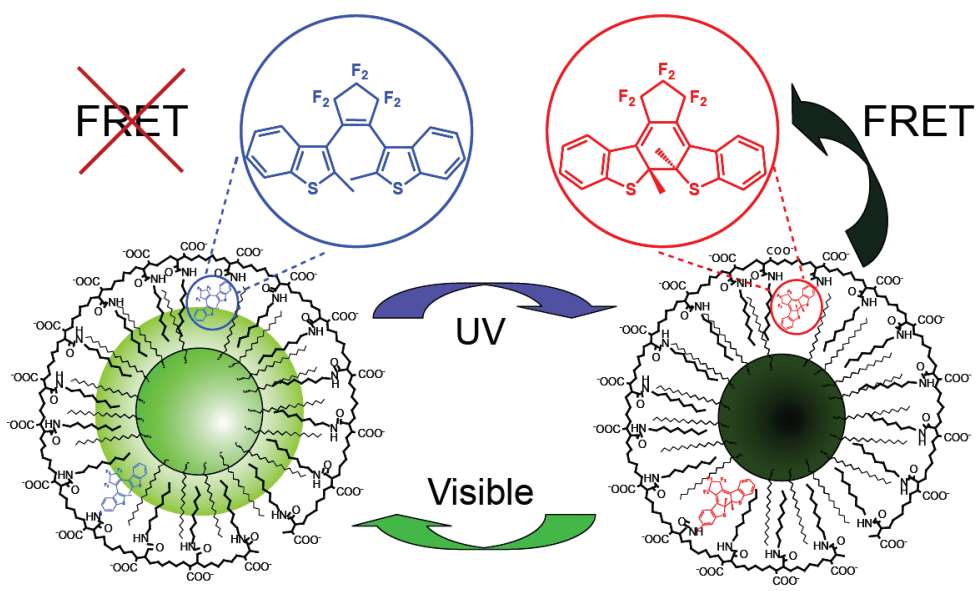

Scheme 1: Photomodulation of psQDs

\subsection{Photoswitchable Quantum Dot (psQD)}

In Section 1.4 the list of prepared polymers includes a series of amphiphilic comb-polymer with different $\mathrm{PCmNH}_{2}$ concentrations ranging from 0 to $6 \%$. This $\mathrm{PC}$ without an extended linker was the first one chosen for studying the modulation of QD fluorescence. The concentrated polymer solutions had a light yellow color in the open form and upon photochromic cyclization changed to dark violet. The majority of experiments in Section 3.1 were performed with small $(\sim 4.1 \mathrm{~nm}$ core diameter) commercial CdSe/ZnS core-shell QDs with octadecylamine ligands, (CZ520, NN-Labs, Fayetteville, AR) and an emission centered at $547 \mathrm{~nm}$. These were coated with the photochromic polymer, thereby transferring the hydrophobic QDs to an aqueous medium. Though various photochromic polymers where prepared the focus of the work was on a psQD prepared with PMA 6PC 70C12. The sample was representative of the other preparations, at the same time its higher PC concentration made it the sample with the highest fluorescence quenching. The use of other polymer or QDs will be specifically noted. 


\subsubsection{Preparation and Purification of psQDs}

The psQD self-assembly is identical to that presented for psNP in Chapter 2. In summary, an interdigitation of the polymer lipophilic chains with the aliphatic chains of the QD drives the coating and the carboxylic groups oriented to the external aqueous medium render the assembled psQD water soluble.

QD samples were precipitated from toluene (solvent supplied by the manufacturer) and resuspended in anhydrous $\mathrm{CHCl}_{3}$. An absorbance spectrum of the solution was taken so as to determine the concentration. A solution of the photochromic polymer was prepared in $\mathrm{CHCl}_{3}$ $[\sim 10 \mathrm{mg} / \mathrm{ml}]$. The solutions were mixed in a glass flask in a determined optimum proportion $(1 \mathrm{mg}$ polymer for $200 \mathrm{pmol}$ of QDs). The reaction was mixed at $65{ }^{\circ} \mathrm{C}$ for $2 \mathrm{~h}$. The solvent was evaporated slowly, and once the sample was dry, SBB $50 \mathrm{mM}$ pH 10 was added in excess. The samples were left overnight with mild stirring, and then passed through $0.2 \mu \mathrm{m}$ inorganic syringe filters. The solution was concentrated to $1 \mu \mathrm{M}$ range using Amicon $100 \mathrm{kDa}$ cutoff filters and clean buffer. Injections of 50 $\mu \mathrm{L}$ were applied to a Superdex 200 analytical column in an HPLC system. Elution was with $50 \mathrm{mM}$ SBB, pH 9, starting with a flow of $50 \mu \mathrm{L} / \mathrm{min}$ for $3 \mathrm{~min}$ and then $20 \mu \mathrm{L} / \mathrm{min}$. The average run time was $70 \mathrm{~min}$, with the psQD fraction collected at $45 \mathrm{~min}$. In some cases a second injection was required to obtain the desired purity. ${ }^{14}$

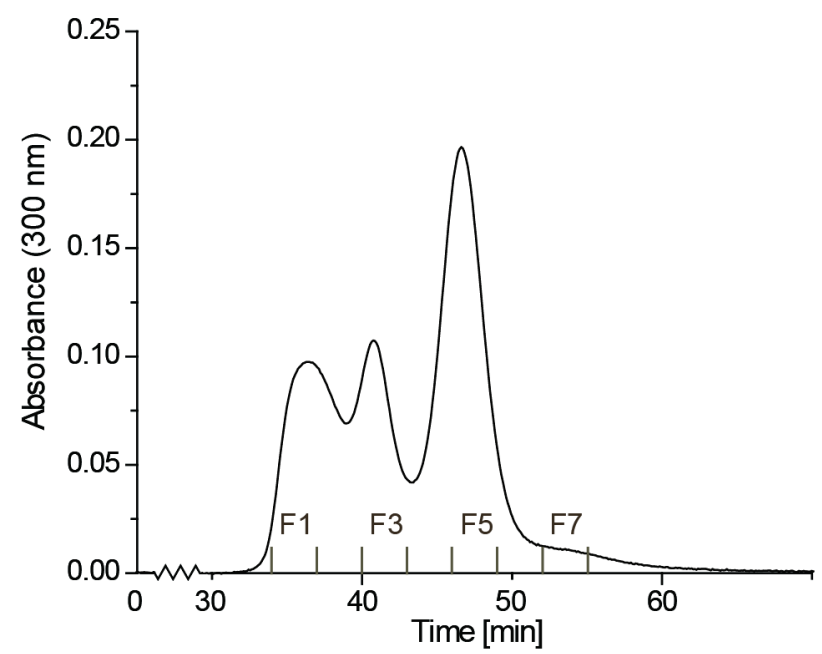

Figure 2: Chromatogram of psQD size-exclusion purification. Absorbance at $300 \mathrm{~nm}$. Fraction 5 represents the psQD peak, while F1 and F3 are polymersomes of different sizes.

The principal impurity was excess polymer forming polymersomes, which had diameters $>30$ $\mathrm{nm}$ according to determinations by dynamic light scattering (DLS). These were separated by a sizeexclusion column selected because of the smaller psQD hydrodynamic diameters of $\sim 15 \mathrm{~nm}$. The purification of the psQDs was essential for the correct measurement of the pcFRET effect described in the following sections. To determine a qualitative influence of polymersomes on the psQDs three samples were prepared (NN-Labs CZ540 coated with PMA 0.7PC 70C12 polymer) of approximately 
the same psQD concentration but with different contents of polymersomes: (i) none, Fraction 5 from Figure 2 (ii) low, Fraction 4 from Figure 2 (iii) high (purification step omitted). Increasing the micellar content led to greater scattering, discernible by apparent absorbance in the $600-650 \mathrm{~nm}$ range. All three samples were irradiated with UV light until no further photoconversion was observed in the absorbance spectra. The fluorescence modulation increased with the polymersome content measuring 10, 12, and 26\% for Pure, Low, and High respectively (Figure 3). The quenching attributable exclusively to pcFRET was $9.5 \pm 1.0 \%$ according to fluorescence lifetime determinations, ${ }^{15}$ in agreement with the steady-state emission of the purified sample. In conclusion, excess polymersomes result in an over-estimation of steady-state fluorescence quenching.
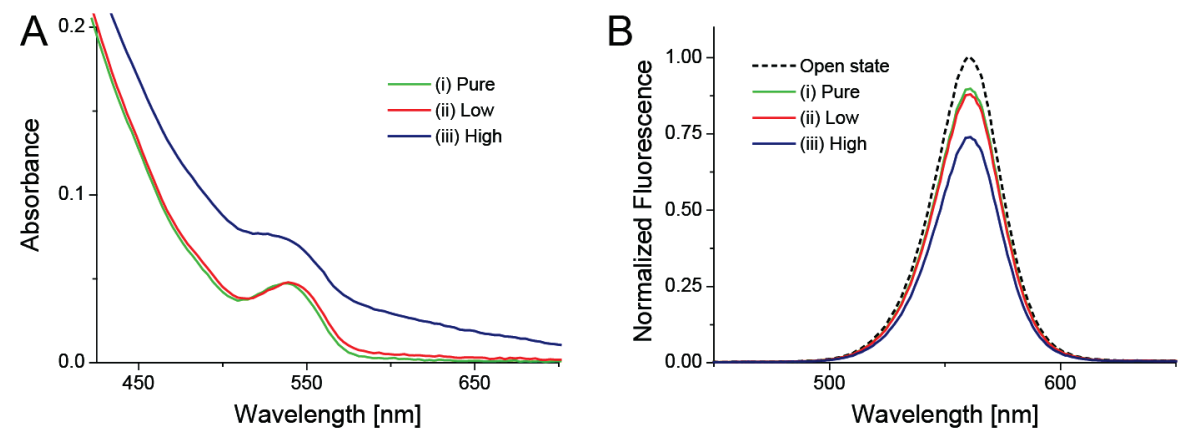

Figure 3: A) Absorbance of psQD samples with varying degrees of polymersome impurifications B) Fluorescence of same samples in the PS state. Dashed line represents the fluorescence of all samples in the open state. Excitation at $400 \mathrm{~nm}$.

The psQD were imaged using TEM microscopy to estimate their diameter. It could be seen that the polymer coating of the QDs led to an increase in the mean diameter from $4.5 \pm 0.7$ to $6.7 \pm 1.3$ $\mathrm{nm}$. The TEM images also showed that the psQDs remained disperse, i.e. did not aggregate, under normal conditions. Previous studies, found in the literature, of systematic comparisons of different techniques for the determination of polymer capped nanoparticle diameters allowed us to estimate a $\sim 15 \mathrm{~nm}$ hydrodynamic radius for the psQDs based on the $7 \mathrm{~nm}$ diameter observed in the TEM images. ${ }^{16,17}$
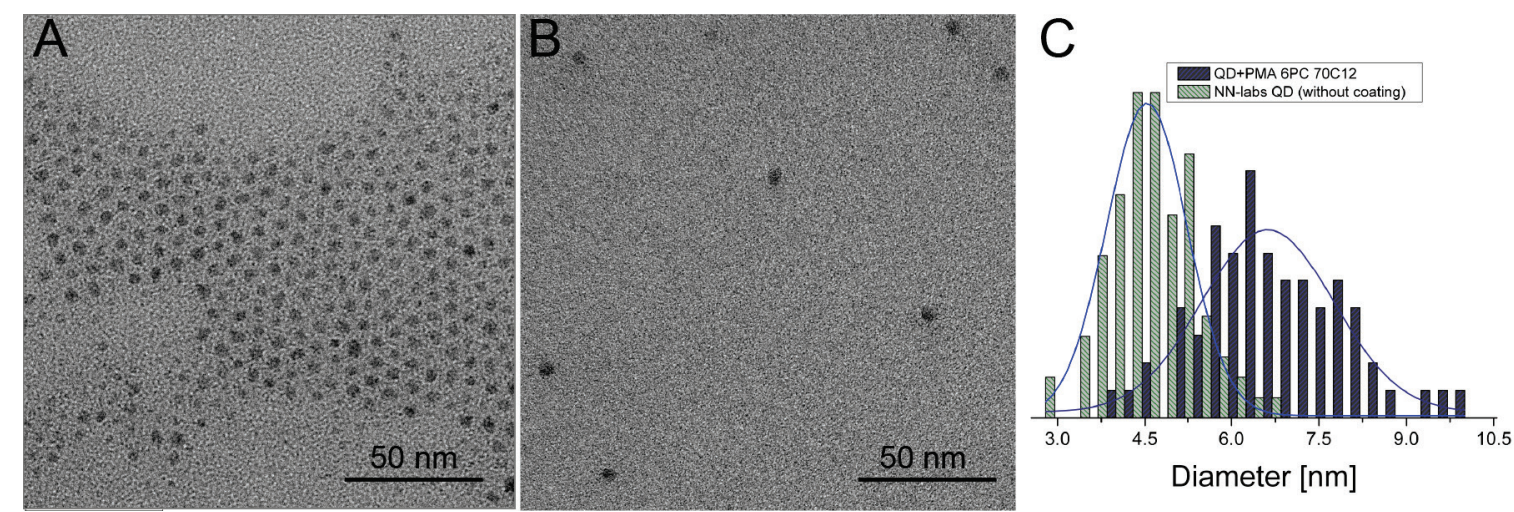

Figure 4: A) TEM of QDs as received from manufacturer B) TEM of psQDs C) Histogram of diameter sizes.

Purified samples of psQDs were stable for $>1$ year upon storage at $4{ }^{\circ} \mathrm{C}$ and at alkaline $\mathrm{pH}(9$ 12). For short periods of time (hours), for example the time necessary to perform EDC coupling, the 
preparations could be transferred to $\mathrm{pH}$ 6.5-7. At lower $\mathrm{pH}(\sim 6)$ aggregation was observed, but was reversed upon rapid alkalinization of the medium.

\subsubsection{Spectral Characterization of psQDs}

The psQDs were studied spectroscopically by absorbance, steady-state fluorescence, and TCSPC-monitored fluorescence decay.

The polymer characterization was presented in Section 1.4. Values for the polymer coating of QDs were obtained by absorbance spectroscopy. The $\mathrm{Abs}_{525}$ of the psQDs in the oPC form permitted an estimation of the QD concentration. The increment in $\mathrm{Abs}_{545}$ upon UV irradiation was assigned exclusively to changes in the relative concentrations of oPC and $\mathrm{cPC}$ (light does not affect the absorption cross-section of the QDs). The concentrations of $\mathrm{cPC}$, and thus of total PC and number of PCs per QD, were obtained from the known differences in extinction coefficients and a $\alpha_{\mathrm{PS}}$ value of 0.20 (see Section 3.2). The smaller $\alpha_{P S}$ than that for the polymer alone (0.22) is a result of the FRET from the QD. ${ }^{18}$ In the PS state the psQD has $17 \mathrm{cPC} / \mathrm{QD}$, using the $\alpha_{\mathrm{PS}}$ I calculate a total of $85 \mathrm{PC} / \mathrm{QD}$. The FRET system for psQDs varies from that presented for the LY-PC FRET pair and will be presented in detail in Section 3.2. The $\mathrm{R}_{\mathrm{o}}$ values were determined (assuming, refractive index $n_{\mathrm{pol}}=$ $1.43, k^{2}=2 / 3$, and donor $\mathrm{QY}=0.22$, Equation 3 Introduction) as QD-oPC $=1.3 \mathrm{~nm}$ and $\mathrm{QD}-\mathrm{cPC}=4.1$ $\mathrm{nm} .{ }^{19}$ The QD-oPC $\mathrm{R}_{\mathrm{o}}$ is so small that it would need to be within the QD core to have any discernible FRET. If the $550 \mathrm{~nm}$ QD-cPC value $(4.1 \mathrm{~nm})$ is compared with the $635 \mathrm{~nm}$ emitting QD $(2.3 \mathrm{~nm})$ the $\mathrm{R}_{\mathrm{o}}$ is almost twice as large and the QD core is smaller, reducing the inaccessible distance. Therefore the choice of the $550 \mathrm{~nm}$ emitting QD was expected to lead to an increase in fluorescence modulation.
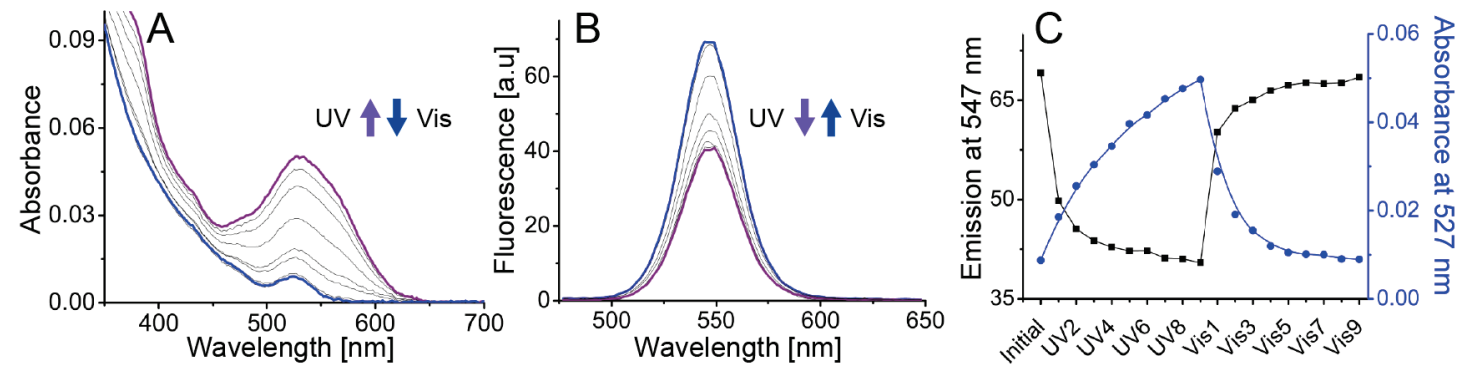

Figure 5: Spectroscopic monitoring of psQDs. Irradiation was with sequential $10 \mathrm{~s}$ pulses. psQD concentration $0.2 \mu \mathrm{M}$. Temperature, $20{ }^{\circ} \mathrm{C}$. (A) Absorbance spectra of the sample undergoing photoconversion from its initial/open state (blue) to a photostationary state (purple) with UV and then returning to the open state with visible irradiation. (B) Fluorescence spectra corresponding to A. Excitation at $400 \mathrm{~nm}$. (C) Absorbance (๑) at $527 \mathrm{~nm}$ after each irradiation step from A. Fluorescence $(\bullet)$ at $547 \mathrm{~nm}$ of each irradiation step from B. ${ }^{14}$

As can be seen in Figure 5 the UV irradiation caused the photocyclization of the PC to the cPC form. This was followed in the absorbance spectra as in increase in the $545 \mathrm{~nm}$ peak, while in the fluorescence spectra the QD emission peak decreases. The kinetics of photocyclization and quenching will be analyzed in Section 3.2. In the photostationary state established by exposure to UV light, the maximal quenching of psQD fluorescence was $41 \%$. This value was obtained with the PMA 6PC 
$70 \mathrm{C} 12$ polymer; samples prepared with polymers containing less PC demonstrated diminished quenching as can be seen in Figure 6. In fact a quenching of $3 \pm 1.5 \%$ was often exhibited by QDs lacking the PC polymer. This was tentatively attributed to photo-oxidation of the QD core induced by UV light in a "susceptible" subpopulation with incomplete passivation shells. ${ }^{20}$ That visible light led to a return to the initial value or slight increase in QD emission, even without PC molecules, can be due to numerous photoactivation mechanisms. ${ }^{21}$ The psQD sample was exposed to longer periods of irradiation (2-fold the required for reaching the photostationary states) and cycled between the two end states. The system was stable and demonstrated no fatigue through 16 cycles with a mean quenching of $34 \pm 2 \%$.
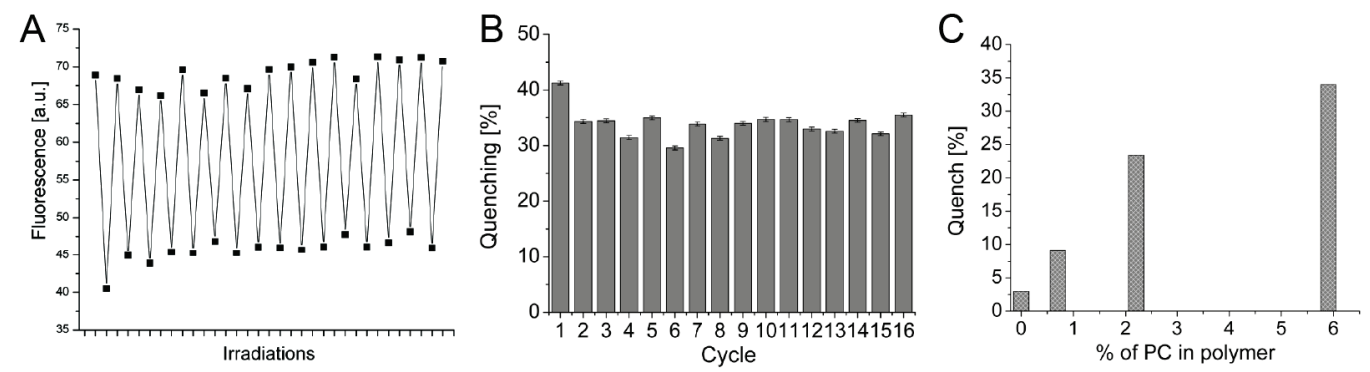

Figure 6: Fluorescence monitoring of psQDs cycled through on-off states. Irradiation pulses of $60 \mathrm{~s}$. psQD concentration: $0.2 \mu \mathrm{M}$. A) Fluorescence at $547 \mathrm{~nm}$ of a sample undergoing photocycling. Excitation, $400 \mathrm{~nm}$. B) Quenching of emission at $547 \mathrm{~nm}$ during each cycle observed in A. C) Mean quenching obtained of psQDs coated with polymers containing different percentages of PC.

Fluorescence lifetime measurements were performed on the psQDs in both the oPC and PS state. The decays were complex, even with the original QDs, and at least 3 exponential components were required to adequately represent the time course of deactivation. The change in mean lifetimes corresponded well with the quenching values obtained by steady state fluorescence. The sample presented in Figure 7 had a mean lifetime $\langle\tau\rangle_{\text {amp }}$ of $9.6 \pm 0.6$ ns for the open state (maximum fluorescence) and of $7.0 \pm 0.4 \mathrm{~ns}$ for the PS state (minimum fluorescence), corresponding to $27 \pm 8 \%$ quenching, in suitable agreement with the $\sim 34 \%$ obtained from the steady state determinations. FRET systems with donors presenting multiple decays are generally represented as $\langle\tau\rangle_{\text {amp }}$ since this quantity reflects the total emission under the decay curve. ${ }^{15}$ Most psQD samples demonstrated a somewhat smaller modulation in time-resolved than in steady state fluorescence. Neither trivial reabsorption of the QD emission by the shell of cPC groups nor the absorbance of the closed cPC form at the excitation wavelength employed ( $400 \mathrm{~nm}$; $\varepsilon$ of oPC and $\mathrm{cPC}, 175$ and $5730 \mathrm{M}^{-1} \mathrm{~cm}^{-1}$ respectively) can fully rationalize this result. QD dark states assigned to charged particles are known, though not fully understood, and may be an additional component in this difference. ${ }^{22}$ 


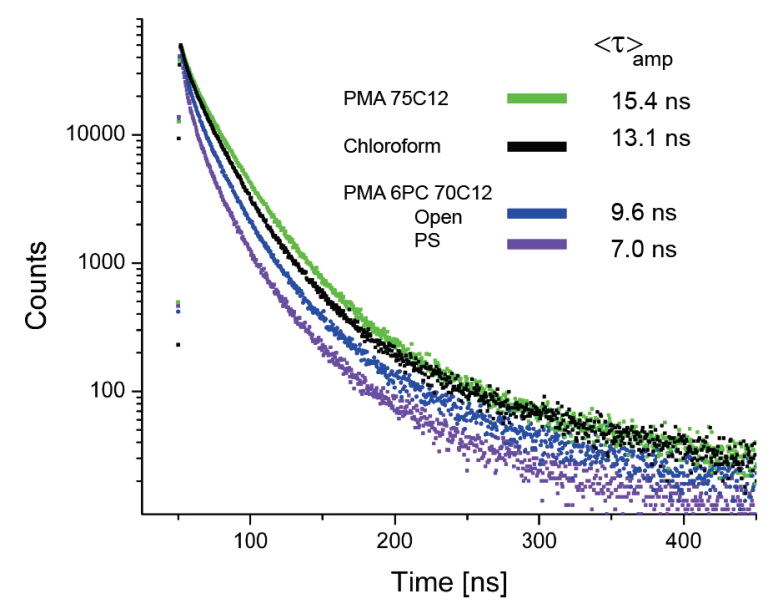

Figure 7: Fluorescence-detected excited state decays of NN-Labs CZ520, CdSe/ZnS QDs in chloroform, coated with PMA 75C12, or coated with PMA 6PC $70 \mathrm{C} 12$ and cycled through the open (visible irradiation)-PS (UV irradiation) states. Emission counts determined at $547 \mathrm{~nm}$.

\subsubsection{Photoconversion Kinetics}

The rates and extents of photoconversion to $\mathrm{CPC}$ and QD quenching were monitored by both UV/Vis absorption and fluorescence spectroscopy after successive UV (340 $\pm 10 \mathrm{~nm})$ and visible $(545 \pm 10 \mathrm{~nm})$ irradiations. The change in absorption was fit well by a monoexponential function $(55 \pm 2$ $\mathrm{s}^{-1}$ ), as observed in Chapter 2 for this class of PC molecules. It must be kept in mind that the apparent rate constant has a photoreversal component due to FRET from the QD.

QD quenching as a function of the amount of acceptor cPC molecules is conceptually complex. The psQD is a system with a single donor with multiple acceptors but as it will be shown not all acceptors are equivalent. ${ }^{14}$ In the next section a model will be presented that adequately describes the psQD quenching.

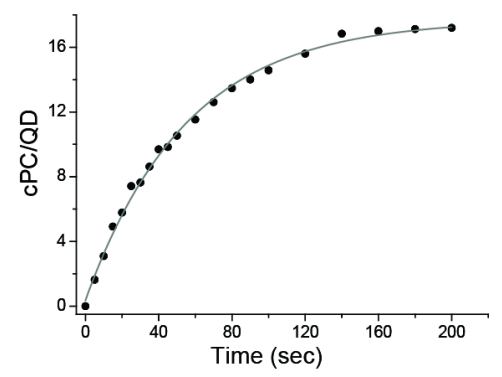

Figure 8. Graph of the evolution of $c P C / Q D$ as a function of $\mathrm{UV}\left(1.1 \mathrm{~mW} \mathrm{~cm}{ }^{-2}\right)$ irradiation time. Fit is monoexponential. 


\section{2 pcFRET Model for psQDs}

The first, and simplest, proposed model system can be visualized as a QD donor surrounded by a shell of cPC acceptors as seen in Scheme 1. The amount of acceptors will vary as UV irradiation is applied until a photostationary stage is reached. If there is a single homogenous shell of PC molecules, such that every PC photocycles at the same rate to an equivalent $\mathrm{cPC}$, than the quenching should be represented by the following equations.

$$
\begin{array}{ll}
\text { Quench } \%=100 * E=100 *(x \gamma /(1+x \gamma)) & \text { Eq. [1] } \\
\gamma=\left(R_{o} / r_{D A}\right)^{6} & \text { Eq. [2] }
\end{array}
$$

where $\mathrm{x}$ is the $\mathrm{cPC} / \mathrm{QD}$ ratio, $\mathrm{r}_{\mathrm{DA}}$ is the mean $\mathrm{D}-\mathrm{A}\left(\mathrm{QD}-\mathrm{cPC}\right.$ ) distance and $\mathrm{R}_{\mathrm{o}}$ is the Förster transfer distance (4.1 $\mathrm{nm}$ for QD-cPC). The FRET parameter $(\gamma)$ represents the FRET efficiency of a donor-acceptor pair, as the efficiency grows the $\gamma$ value increases. Figure 9 clearly shows that the fit to Equation 1 is inadequate. The psQD demonstrated high quenching at short irradiation times (low $\mathrm{cPC}$ ) and then an asymptotic behavior as $\mathrm{x}$ increased. This quenching dynamics was consistently observed with all psQDs, independently of the QD or polymer chosen to prepare the psQD. Therefore, the model presented below, elaborated for the previously mentioned $\mathrm{ps} \mathrm{QD}$, is presumed to be applicable to all psQDs.

An increased level of complexity was required to adequately fit the data. It must be assumed that the $\mathrm{cPC}$ acceptors were not homogenous, but were in fact heterogeneous in terms of FRET capability. This could be due to distance and/or orientation effects. ${ }^{19}$ A two component model is the simplest representation of the heterogeneous population.

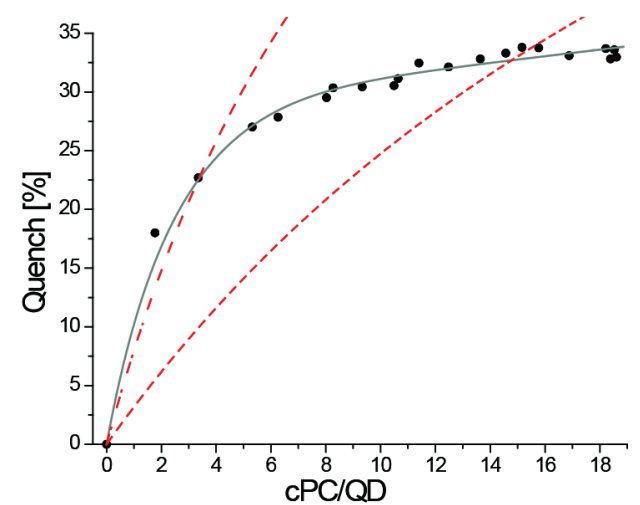

Figure 9: Quenching of QD fluorescence as a function of cPC/QD. Grey Fit corresponds to Equation 3 while dashed red lines correspond to attempted fits with Equation 1.

By definition two classes $(1,2)$ of cPC acceptors are proposed, the only distinction being that class 1 was defined as the more FRET efficient. The assumption was that the inherent photochromic parameters were the same for both populations such that in the absence of FRET, $\alpha_{\mathrm{PS}}$ for the two PC populations was $\sim 0.22$ upon exposure to $\mathrm{UV}(340 \mathrm{~nm})$ irradiation. Ultraviolet light leads to photocyclization of $\mathrm{oPC}$ as well as photoreversion of $\mathrm{cPC}$ to $\mathrm{oPC}$ due to direct excitation and 
indirectly via pcFRET $(\mathrm{QD} \rightarrow \mathrm{cPC})$. Visible light induces photoreversion of $\mathrm{cPC}$ by direct excitation as well as through pcFRET. The photoreversion due to FRET is important since the two acceptors differ in their number, $n_{1,2}$, but more importantly in their FRET parameter $\gamma_{1,2}$. The FRET parameter can vary due to a change in $\mathrm{r}_{\mathrm{DA}}$ (distance) and/or $\mathrm{R}_{\mathrm{o}}$ (orientation). The fractional photoconversion to cPC, $\alpha[\mathrm{t}]$, is different for each class due to the different FRET efficiencies. A higher FRET efficiency results in a higher probability of deactivating an excited QD, but this in turn potentiates the cycloreversion and thus depopulates the closed state. ${ }^{23}$ The correct fit is now described by:

$$
\begin{aligned}
& \text { Quench } \%=100 *\left(E_{1}[t]+E_{2}[t]\right) ; \\
& E_{1}[t]=\frac{c_{1}[t] \gamma_{1}}{1+c_{1}[t] \gamma_{1}+c_{2}[t] \gamma_{2}} ; E_{2}[t]=\frac{c_{2}[t] \gamma_{2}}{1+c_{1}[t] \gamma_{1}+c_{2}[t] \gamma_{2}}
\end{aligned}
$$

Where $c_{1,2}$ is the number of $\mathrm{cPC} / \mathrm{QD}$, and the $\mathrm{oPC} \rightarrow \mathrm{cPC}$ time course is represented by the following differential equation.

$$
c_{i}^{\prime}[t]=k_{o} Q_{o c, u v} \rho\left(n_{i}-c_{i}[t]\right)-k_{c} Q_{c o, u v} c_{i}[t]-k_{Q D} Q_{c o, v i s} E_{i}[t] \quad \text { Eq. [4] }
$$

in which $n_{1,2}$ is the total number of PC/QD of each class and the rate constants $k_{\mathrm{o}}, k_{\mathrm{c}}$, and $k_{\mathrm{QD}}$, for the forward, reverse photoreactions, and the QD-induced (via pcFRET) reverse reaction respectively, are products of the known irradiance and absorption cross-sections. The corresponding quantum efficiency values $Q_{\mathrm{oc}, \mathrm{uv}}, Q_{\mathrm{co}, \mathrm{uv}}, Q_{\mathrm{co} \text {,vis }}$, and $\gamma_{1}, \gamma_{2}, n_{1}$ and $n_{2}$ are fitted variables $\left(n_{1+} n_{2}=85\right)$. Though one variable is reduced since $\mathrm{Q}_{\mathrm{oc}, \mathrm{uv}}$ and $\mathrm{Q}_{\mathrm{co}, \mathrm{uv}}$ are dependent:

$$
Q_{c o, u v}=\rho\left(\frac{1-\alpha_{\mathrm{PS}}}{\alpha_{\mathrm{PS}}}\right)\left(\frac{\varepsilon_{o P C, u v}}{\varepsilon_{c P C, u v}}\right) Q_{o c, u v}
$$

Where $\rho$ is the fraction of the photocyclization-competent antiparallel isomer of oPC. The relation represented by Equation 5 results from assuming that $\alpha_{\mathrm{PS}}=0.22$ in absence of FRET.

The FRET efficiencies $E_{1}$ and $E_{2}$, and thus the extent of QD quenching, vary in time and are interdependent with each other through the $c_{1}$ and $c_{2}$ time courses. A fitting routine was developed that simultaneously fit the photoconversion to $\mathrm{cPC}\left(c_{1,2}\right)$ and the QD quenching (Quench\%) as a function of the UV irradiation dose using the FindMinimum routine of Mathematica (Wolfram Research). ${ }^{24}$

Very good fits to the data were achieved and parameter convergence was consistent between various experiments. The polymer preparations with different PC content had kinetic properties consistent with the assumption of a common photoconversion-FRET mechanism and set of rate constants.

Interconversion between the antiparallel and parallel isomers of the open form $\mathrm{PC}$ is slow on the time scale of the experiments. ${ }^{25}$ If this statement does not hold true, appropriate modifications to the model can and should be made. 
Table 1: Analysis of the UV-induced photoconversion and QD quenching kinetics

\begin{tabular}{|c|c|c|c|c|c|c|c|c|}
\hline & & $Q_{o c}$ & $Q_{c o, u v}$ & $Q_{c o, v i s}$ & $n_{1}$ & $n_{2}$ & $\gamma_{1}$ & $\gamma_{2}$ \\
\hline \multirow[t]{2}{*}{$p s Q D$} & & 0.52 & 0.17 & 0.05 & 4 & 81 & 1.5 & 0.01 \\
\hline & $k_{\mathrm{o}}$ & $\begin{array}{l}k_{\mathrm{c}} \\
s^{-1}\end{array} k_{\mathrm{QD}}$ & $E_{1, \mathrm{PS}}$ & $E_{2, \mathrm{PS}}$ & $\alpha_{1, \mathrm{PS}}$ & $\alpha_{2, \mathrm{PS}}$ & $\left(\frac{r_{\mathrm{DA}}}{R_{\mathrm{o}}}\right)_{1}$ & $\left(\frac{r_{\mathrm{DA}}}{R_{\mathrm{o}}}\right)_{2}$ \\
\hline$p s Q D$ & 0.01 & $\begin{array}{lll}0.08 & 0.8\end{array}$ & 0.32 & 0.10 & 0.03 & 0.21 & 0.94 & 2.2 \\
\hline
\end{tabular}

psQD: CdSe/ZnS QDs (CZ520, NN-Labs) coated with PMA 6 PC 70 C12.

The 2 class acceptor model, as a simplification of the heterogeneous acceptor model, can account for the shape of the data in Figure 9. The Class 1 acceptors are very efficient and account for the high quenching at low concentrations but overall are a small percentage of the total PC. The Class 2 acceptors dominate the $\mathrm{cPC}$ conversion time course but are not very efficient in FRET, accounting for the asymptotic tail observed in the data.

A key photochemical feature was the pronounced effect of FRET on the extent of photoconversion of the two classes. The large absorption cross-section of the QD accounts for most of the $\mathrm{Abs}_{340}$ and leads to the condition where $\mathrm{k}_{\mathrm{QD}}>>\mathrm{k}_{\mathrm{o}}, \mathrm{k}_{\mathrm{c}}$. Equation 4 predicts that, Class 1 acceptors with high FRET efficiency will be kept at a low $\alpha_{\mathrm{PS}}$, while the Class 2 is reduced but not nearly as much. The mathematical model allows for the estimation of the $\alpha_{P S}$ of a PC engaged with a nanoparticle through a near field interaction (in this case FRET), which in previous works was assumed to be similar to the free dye. ${ }^{26}$

The absorbance spectra yielded $\sim 17 \mathrm{cPC} / \mathrm{psQD}$ in the PS state and taking into account the complex photophysical model an aggregate $\alpha_{\mathrm{PS}}$ value of 0.20 was determined. Therefore the calculated total number of photoconversion-competent molecules was $\sim 85$. Some care must be taken when considering these numbers, since the total number of PC molecules was pre-assigned in the fitting. In later experiments, seen in Section 3.3, an internal standard allowed for the determination of total PC, and the observed results confirmed the methodology. Small changes in the total number of PC does not modify the values to the point where the interpretation of the results is altered.

As discussed before the two acceptor populations could in principle exhibit different values of $\gamma$ due to orientation and/or distance factors. The next section proposes a physical model to explain this difference.

\subsubsection{Physical Model Describing the pcFRET System}

Class 1 and Class 2 cPC differ in (A) FRET efficiency $\left(\gamma_{1}>\gamma_{2}\right)$ by definition and (B) the number of molecules: $n_{1}<n_{2}$ by data analysis. The FRET efficiency is favored for cPC groups either situated closer to the QD surface and/or in an orientation more favorable for energy transfer by virtue 
of an absorption transition moment positioned more normal than parallel to the QD surface. ${ }^{19}$ The second case would be manifested by a lower value of the FRET orientation factor $k^{2}$ proportionally decreasing $\mathrm{R}_{\mathrm{o}}$. Various possibilities or combinations of possibilities could account for the separation into two classes of the cPC acceptors.

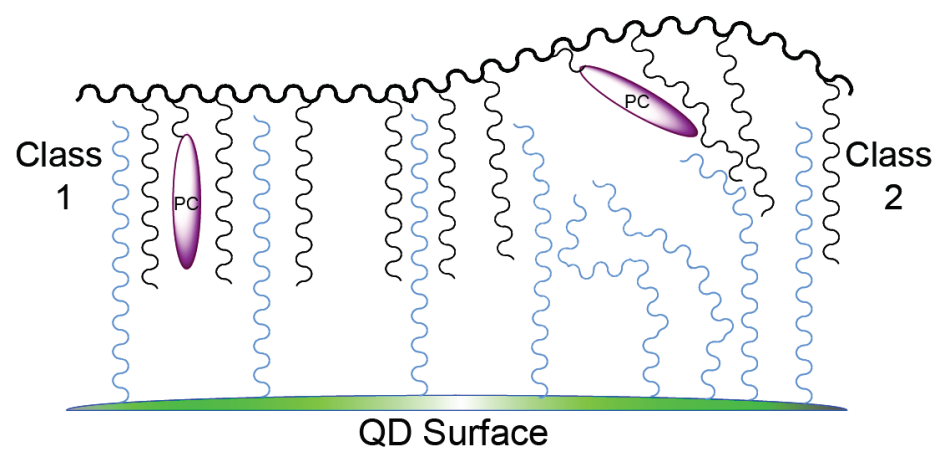

Scheme 2: Heterogeneity of distance and orientation of PC moieties, the FRET acceptors, to the QD surface. The FRET efficiency and photoconversion rate and extent are affected by the PC positioning. In this example, class 2 molecules are shown both further and oriented less favorably (for FRET) to the QD surface. The pronounced curvature of the QD surface on this size scale and the consequent tilting and splaying of the aliphatic chains are not depicted. ${ }^{14}$

Scheme 2 depicts one of these proposed situations. Class 1 PC groups are embedded in proximal regions of the "comb" with efficiently stacked alkyl chains, whereas class 2 PC molecules are shown in local regions with turbulent alkyl chains. These locations presumably exhibit an insertion "locus" more removed from the surface of the QD core. Regions of inefficient interdigitation of the alkyl chains would be forced away from the QD surface into class 2 "loci". Assuming saturation of the $4.1 \mathrm{~nm}$ QD surface with octadecylamine ligands with the accepted packing density of 18.5 $\AA^{2} /$ molecule, the number of aliphatic chains/QD would be $\sim 300 .{ }^{27}$ The polymer presents $6 \% \mathrm{PC}$ and $70 \% \mathrm{C} 12$ and the estimated number of $\mathrm{PC} / \mathrm{QD}$ was 85 resulting in $\sim 1400$ monomer groups or 35 polymer chains per QD. The large number of polymer chains to QD ligands could allow for the exploitation of surface defects, where QD ligands are not present, to place the PC very close to the surface. Finally polymers with backbone lengths of $\sim 40$ monomers may not form a homogeneous cap on the QD surface; one must assume the existence of cross-over sites, which would necessarily constitute additional loci of PC displacement to the exterior.

With the values obtained from the fittings the mean distance of each class of acceptor was calculated. I assumed point emission from the center of the QD and calculate $\mathrm{r}_{\mathrm{DA}}=3.8 \mathrm{~nm}$ for Class 1 and $8.8 \mathrm{~nm}$ for Class 2. This estimate can be refined by the analysis presented by Grecco $e a^{28}$ where the formulation for calculating the distance of a FRET acceptor to a QD donor surface is:

$$
\Delta r_{i}=\left(R_{o} * \sqrt[6]{\frac{1}{\gamma_{i}}}\right)-r_{Q D}\left(n_{Q D} / n_{p o l}\right)^{2 / 3}
$$


The refractive index term corrects for virtual dilation of the distance due to propagation in the dense QD semiconductor medium $\left(n_{\mathrm{QD}}=2.55, n_{\mathrm{pol}}=1.43\right)$. With $r_{\mathrm{QD}}=2.1 \mathrm{~nm}, \mathrm{a} \Delta r_{1}=0.75 \pm 0.05 \mathrm{~nm}$ for the Class 1 acceptors is calculated. These values are compatible with the size of the psQD placing the acceptors well within the interior of the polymer shell. A corresponding calculation for acceptor population 2 yields $\Delta r_{2}=\sim 6 \mathrm{~nm}$, an unrealistically large value attributable to the uncertainty in how to interpret the very low value of $\gamma_{2}$. Assuming that the difference in $\gamma_{1}$ and $\gamma_{2}$ is due exclusively to orientation, such that $\Delta r_{1}=\Delta r_{2}=0.75$ and $k_{1}^{2}=2 / 3, k_{2}^{2}$ would be 0.005 , implying a very unfavorable orientation for transfer. More likely, $k_{2}^{2} / r_{D A 2}<<k_{1}^{2} / r_{D A 1}$ such that both distance and orientation effects are involved. From an operational standpoint, the assumption of two classes of acceptors, distinguishable by their FRET properties, accounts very satisfactorily for the observed properties of the psQD system.

\subsection{Dual-color psQDs}

In this section a new structural design is presented that creates a psQD with a secondary dye (Alexa Fluor647 cadaverine) acting as an internal standard which allows for dual-color, ratiometric sensing and imaging. ${ }^{18}$ The psQD remains similar in size $(\sim 6 \mathrm{~nm}$ diameter by EM, a $\sim 14 \mathrm{~nm}$ hydrodynamic diameter), does not exhibit fatigue, and the QD modulation is improved by using a linker PC. The ratiometric signal changes by $\sim 100 \%$ and allows selective detection. The detection can be applied in the steady-state as well as in the ns regime, in the latter case exploiting the different QD and Alexa647 fluorescence decay kinetics.

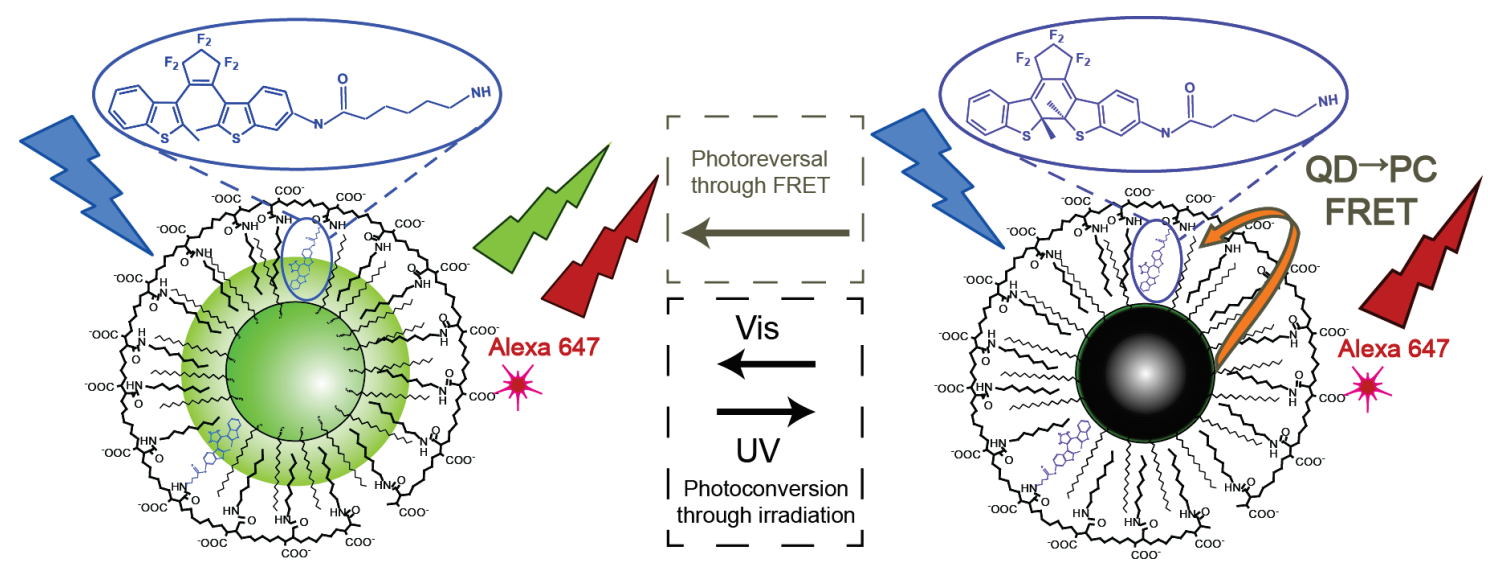

Scheme 3: Photomodulation of dual-color psQDs

\subsubsection{Preparation of Dual-color psQDs}

The polymer characterization and synthesis was presented in section 1.4.3. The dual-color psQD was coated with PMA 7PCahx 75C12 0.3Al polymer (for every 1000 original maleic anhydride monomers there were 750 dodecylamine chains, $\sim 70 \mathrm{PCahxNH}_{2}$ molecules and 3 Alexa647). The $\mathrm{PCahxNH}_{2}$ is a $\mathrm{PC}$ with a 6 carbon linker terminating in a primary amine. ${ }^{18}$ This linker positions the $\mathrm{PC}$ closer to the QD than in the first psQD as well as increasing the yield of conjugation to the 
polymer. The linker did not affect the photophysical properties of the PC, therefore the same considerations and values were used for the analysis.

A different version of green emitting QDs from those used in Section 3.1 was selected for the experiments in this section. The QDs were $\mathrm{CdSe} / \mathrm{CdS} / \mathrm{ZnS}$ core-shell-shell nanoparticles (Series A CSS $540 \mathrm{~nm}, \mathrm{CAN} \mathrm{GmbH}$, Hamburg) emitting at $540 \mathrm{~nm}$ and rendered soluble in organic solvents by lipophilic surface ligands (TOPO/TOP/HDA). The coated QDs were prepared and purified the same as the first psQDs. ${ }^{14}$ The hydrophilic Alexa647 and pendant carboxylic groups were presumed to extend into the aqueous medium, conferring a negative surface charge and solubility to the nanoparticle. The final construct was denoted a dual-color psQD (dual-color photoswitchable quantum dot). The properties and data presented were determined in $50 \mathrm{mM} \mathrm{SBB}, \mathrm{pH} 9.0$, under aerobic conditions. The dual-color psQD presented the same stability as the psQDs.

\subsubsection{Spectral properties of Dual-Color psQDs}

The phase transfer shifted the peak of the QD emission to $550 \mathrm{~nm}$ and led to a reduction of the quantum yield from 0.70 in $n$-heptane to 0.26 , a value similar to that reported for other polymer coated QDs. $^{29}$

The photochrome composition of the dual-color psQD was determined by resolving the absorbance spectrum into its constituent contributions. The $\mathrm{Abs}_{525}$ with the $\mathrm{PC}$ in the open form was assumed to originate exclusively from the QD, thus permitting an estimation of the nanoparticle concentration. The increment in $\mathrm{Abs}_{540}$ upon UV irradiation was assigned to changes in the relative concentrations of oPC and $\mathrm{cPC}$. The concentrations of $\mathrm{cPC}$, and thus of total $\mathrm{PC}$ and number of PCs per QD, were obtained from the known differences in extinction coefficients and the $\mathrm{CPC}$ fraction in the photostationary state $\left(\alpha_{\mathrm{PS}}\right)$. The $\mathrm{Abs}_{650}$ originated solely from the Alexa647 and thus an Alexa647/QD ratio could also be computed. The total number of PC/QD can be confirmed by the known ratio of $\mathrm{PC} / \mathrm{Alexa} 647$ in the original polymer, which will remain unchanged when coating the QD. In interpreting the data, a random distributions of dyes on the polymer and of the polymer on the QD was assumed. The calculations yielded mean values of 1.2 \pm 0.1 Alexa647 and 35.0 1.0 PCahx per QD, corresponding to $\sim 400-500$ monomers of polymer (or $\sim 10-13$ polymers) per QD. The polymer content was less than half of the psQD presented in the previous section due to improvements in coating strategy and that the selected QDs are of better quality. The more efficient packing was reflected in the ease of QD coating and phase transfer. From the Alexa/QD ratio, a fractional distributions for QDs with $0,1,2$ and $>2$ Alexa647 would be $0.30,0.36,0.22$, and 0.12 , respectively.

Figure 10 shows the most pertinent spectral properties of the individual components of the dual-color psQD. The latter exhibited two emission wavelengths; $550 \mathrm{~nm}$ and $666 \mathrm{~nm}$, corresponding to the QD and Alexa647, respectively. Both emissions could be elicited over a broad excitation band corresponding to the QD. In the experiments described hereafter the selected excitation wavelengths 
of $400 \mathrm{~nm}$ and $600 \mathrm{~nm}$ were optimal in that they correspond to minima in the PCahx absorbance. As demonstrated QDs can act as FRET donors, in this case not only to the PCahx groups but also to the Alexa647, which will thus exhibit emission upon excitation throughout the QD excitation band. The computed $\mathrm{R}_{\mathrm{o}}$ parameters are: QD-oPC, $1.3 \mathrm{~nm}$; QD-cPC, $4.1 \mathrm{~nm}$; QD-Alexa647: $4.1 \mathrm{~nm}$, assuming 2/3 for $\kappa^{2}$. The similar values for $\mathrm{cPC}$ and the Alexa647 result from compensation of the reduced positional overlap by the much larger extinction coefficient of Alexa647 (15-fold at their respective peaks). ${ }^{19}$

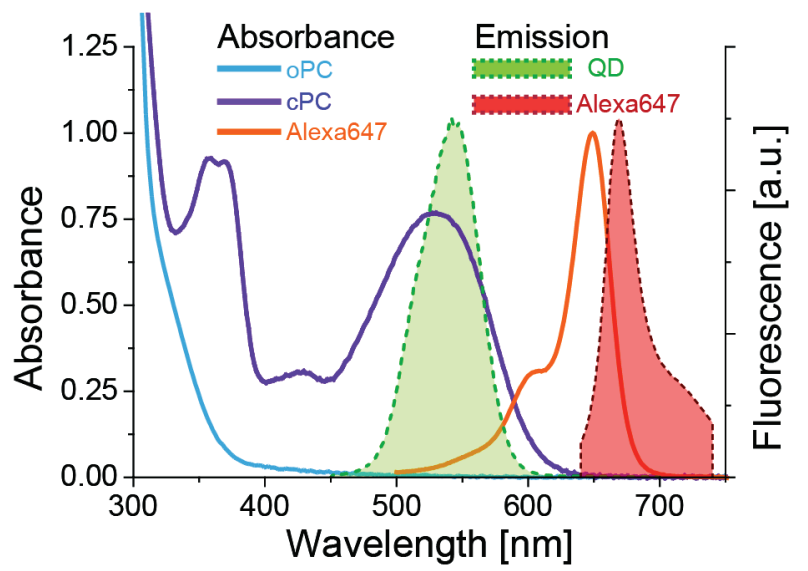

Figure 10: Superposition of absorbance (solid lines) and emission (filled areas) spectra of PC, QD, and Alexa647, demonstrating the PC spectral overlap with the QD but not with the Alexa647. The spectra are normalized by their peak values.

Reversible photoswitching of the PC probe in the dual-color psQD was achieved by alternating irradiation with UV light $\left(340 \pm 10 \mathrm{~nm}, 1.1 \mathrm{~mW} \mathrm{~cm}^{-2}\right)$ and visible/green light (545 $\pm 10 \mathrm{~nm}$, $\left.6.2 \mathrm{~mW} \mathrm{~cm}^{-2}\right)$. Sequential short pulses $(\sim 10 \mathrm{~s})$ of either UV or visible light were utilized to achieve the two photostationary states. The changes in the absorbance and fluorescence spectra of the dual-color psQD upon passing from the open to the closed states are shown in Figure 11. The photostationary state of $\mathrm{PCahxNH}_{2}$ conjugated to the polymer corresponds to a $22 \%$ conversion $\left(\alpha_{\mathrm{PS}}=0.22\right)$. The photoconversion of the dual-color psQD is more complex, due to the existence of two subpopulations of cPC differing in FRET efficiency, as seen in the FRET model (Section 3.2). Thus, the photostationary state of the psQD established by UV irradiation corresponds to a lower $\alpha_{\mathrm{PS}}(0.185)$ than in the case of the free or polymer-conjugated PC.

The maximum observed quenching of fluorescence at the $550 \mathrm{~nm}$ peak upon exposure to UV light was $52 \%$ (mean $48 \pm 2 \%$ ). Irradiation with visible light restored the fluorescence to the initial state. When excited directly the Alexa647 standard was unaffected by the photoconversion of the PC, although a slight photobleaching was evident upon prolonged irradiations. When Alexa647 emission was perceived through sensitized emission ( $400 \mathrm{~nm}$ excitation) a decrease of $15 \%$ was observed in the PS state. 

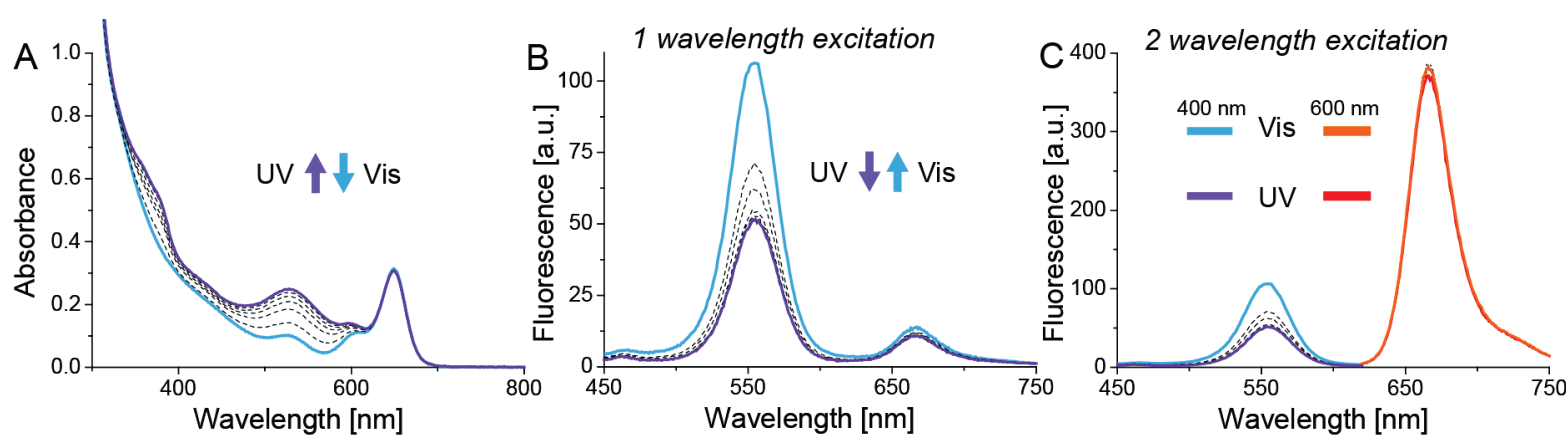

Figure 11: Spectroscopic monitoring of dual-color psQD. Irradiation was UV: 340 (irradiance $1.1 \mathrm{~mW} \mathrm{~cm}^{-2}$ ) and Vis: 545 (irradiance $6.2 \mathrm{~mW} \mathrm{~cm}^{-2}$ ). Temperature, $20^{\circ} \mathrm{C}$. (A) Absorbance spectra (B) Fluorescence spectra of dual-color psQD with excitation at $400 \mathrm{~nm}$. (C) Fluorescence spectra of dual-color psQD with excitation wavelength at $400 \mathrm{~nm}$ for QD detection and $600 \mathrm{~nm}$ for Alexa647 detection. ${ }^{18}$

The fluorescence decay kinetics of the dual-color psQD in different states were measured to confirm that the observed photomodulation was due to FRET. The decays of the parent QD and psQD were complex (Figure 12), requiring four exponential components for the QD (and two for the Alexa647) to adequately represent the time course of excited state deactivation.
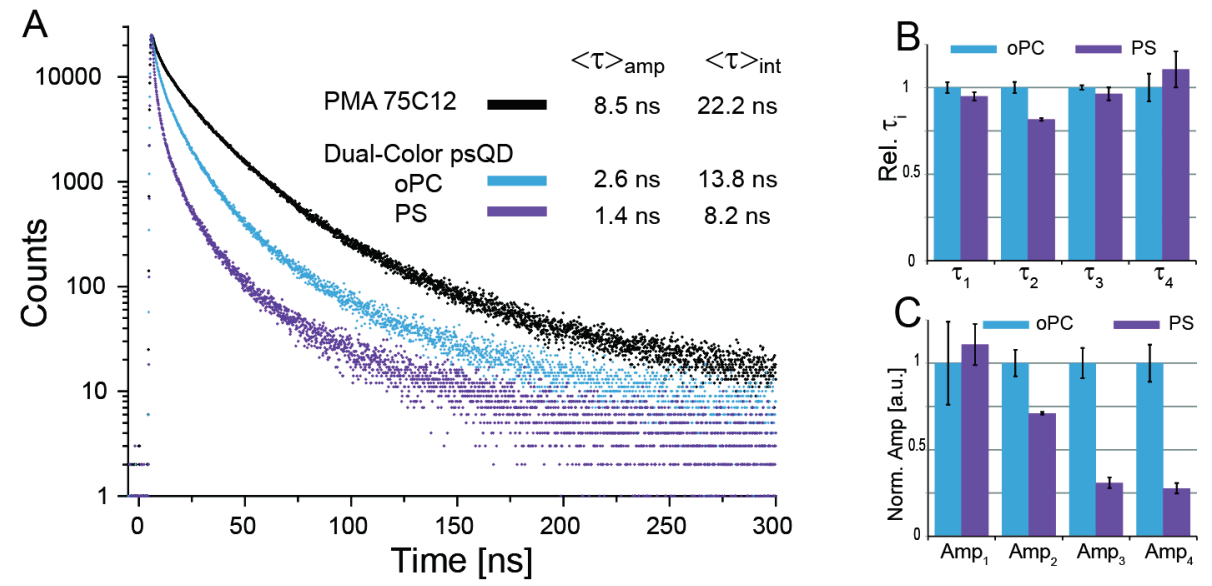

Figure 12: (A) Fluorescence-detected excited-state decays determined at $550 \mathrm{~nm}$. (B) Individual decay constants normalized by the values for the oPC state. (C) Individual amplitudes, normalized by the oPC values.

The psQD time-resolved decays of samples undergoing photoconversion cycles are best interpreted by examining the individual lifetimes and amplitudes of the 4 required eigenvalues. The values are presented in Table 2 and the graphical representation can be seen in Figure 12B,C. The amplitudes were converted to a common absolute scale by dividing each amplitude by $\sum \mathrm{Amp}_{\mathrm{i}}, \mathrm{counts}^{\bullet} \tau_{\mathrm{i}}$ and multiplying by the steady-state intensities. 
Table 2: Individual Lifetimes and Amplitudes of Eigenvalues of Dual-Color psQDs in the Open and PS state.

\begin{tabular}{lcccc}
\hline \multicolumn{1}{c}{ Open } & 1 & 2 & 3 & 4 \\
\hline Lifetime [ns] & $0.48 \pm 0.02$ & $3.8 \pm 0.1$ & $12.7 \pm 0.2$ & $60 \pm 5$ \\
Amplitude & $1.0 \pm 0.2$ & $0.25 \pm 0.02$ & $0.10 \pm 0.01$ & $0.007 \pm 0.001$ \\
\hline \multicolumn{1}{c}{ PS } & & & & \\
\hline Lifetime [ns] & $0.45 \pm 0.01$ & $3.12 \pm 0.03$ & $12.2 \pm 0.5$ & $66 \pm 6$ \\
Amplitude & $1.1 \pm 0.1$ & $0.17 \pm 0.01$ & $0.03 \pm 0.005$ & $0.002 \pm 0.001$ \\
\hline
\end{tabular}

The individual four decay constants did not vary systematically during photocycling but the amplitudes of the longer lifetimes decreased by 30,71 , and $68 \%,\left(\tau_{2,3,4}\right.$ respectively $)$ upon photocyclization. Two derived global means: amplitude weighed lifetime, $\langle\tau\rangle_{\mathrm{amp}}$ and intensity weighed lifetime, $\langle\tau\rangle_{\text {int }}$ (Table 3) can also provide additional information. In fact, $\langle\tau\rangle_{\text {amp }}$ diminished by $47 \pm 3 \%$ $(2.6 \rightarrow 1.4 \mathrm{~ns})$ in 3 successive $\mathrm{oPC} \rightarrow \mathrm{cPC}$ transitions, a value identical to that obtained from the steady-state emission and also indicative of a lack of undetected dark states. ${ }^{30}$ The corresponding change in $\langle\tau\rangle_{\text {int }}$ was smaller (40\%).

Table 3: Amplitude weighed mean lifetime $\langle\tau\rangle_{\text {amp }}$ and intensity weighed mean lifetime $\langle\tau\rangle_{\text {int }}$ of the dual-color psQD and controls. Lifetime values in ns.

\begin{tabular}{cccc}
\hline $\begin{array}{c}\text { QD } \\
\text { detection }^{\mathrm{a}}\end{array}$ & $\begin{array}{c}\text { polymer } \\
\text { coated }^{\mathrm{c}}\end{array}$ & $\begin{array}{c}\text { Dual-color } \\
\text { psQD oPC }\end{array}$ & $\begin{array}{c}\text { Dual-color } \\
\text { psQD PS state }\end{array}$ \\
\hline$\langle\tau\rangle_{\mathrm{amp}}$ & $8.5 \pm 0.1$ & $2.6 \pm 0.5$ & $1.4 \pm 0.1$ \\
$\langle\tau\rangle_{\text {int }}$ & $22.2 \pm 0.2$ & $13.8 \pm 0.2$ & $8.2 \pm 0.2$ \\
\hline \hline $\begin{array}{c}\text { Alexa647 } \\
\text { detection }\end{array}$ & in Solution & $\begin{array}{l}\text { Dual-color } \\
\text { psQD oPC }\end{array}$ & $\begin{array}{c}\text { Dual-color } \\
\text { psQD PS state }\end{array}$ \\
\hline$\langle\tau\rangle_{\mathrm{amp}}$ & $1.28 \pm 0.01$ & $1.18 \pm 0.01$ & $1.14 \pm 0.01$ \\
$\langle\tau\rangle_{\text {int }}$ & $1.17 \pm 0.06$ & $1.06 \pm 0.05$ & $1.02 \pm 0.02$ \\
\hline
\end{tabular}

[a] Excitation with nanoLED N-320 nm and detection at $550 \mathrm{~nm}$. [b] Excitation with nanoLED N-560 nm source and detection at $660 \mathrm{~nm}$. [c] CANdot ${ }^{\circledR}$ CSS $540 \mathrm{~nm}$ QDs coated with amphiphilic polymer lacking PC or Alexa probe.

There was a significant decrease in the $\langle\tau\rangle_{\text {amp }}$ when comparing QDs coated with an amphiphilic polymer lacking PC to the dual-color psQD system. Previous investigations have rationalized this effect in terms of FRET and/or screening processes but the evidence points to coating efficiencies playing a larger role in the system as mentioned in Section 2.2. A polymer that only contains pendant alkyl chains will coat more efficiently than one carrying voluminous dyes such as PCahx and Alexa647. The lower value of the final psQD indicates the presence of surface traps equilibrating with the radiative process of hole-electron recombination and/or the presence of defects and ligands contributing to non-radiative pathways. ${ }^{30}$ The Alexa647 probe exhibited a slightly shorter lifetime when conjugated to $\mathrm{psQD}$ than in solution but the sensitivity to PC photocycling was 
negligible. The effect observed in Chapter 2, of an extended lifetime due to sensitized emission was avoided by using an excitation wavelength that only excited the Alexa647.

\subsubsection{Ratiometric Cycles}

An Emission Ratio (ER) is defined as the Alexa647 emission at $666 \mathrm{~nm}$ divided by the QD emission at $550 \mathrm{~nm}$. Alexa647 can be excited selectively from 580 to $660 \mathrm{~nm}$. Thus, the ER is adjustable by selection of the excitation wavelengths. Using a single excitation wavelength $(400 \mathrm{~nm})$, the ER changed from 0.13 to 0.22 (66\% change). By exciting both probes individually (QD at $400 \mathrm{~nm}$, Alexa647 at $600 \mathrm{~nm})$, the ER changed from 4.0 to 8.0 (100\% change).

The dual-color psQD were cycled through the open-PS states by alternating exposure to UV and visible light and monitoring the QD and Alexa647 emissions (Figure 13). The first irradiation with UV led to a significant decrease in the Alexa647 while subsequent cycles produced a more moderate decrease indicative of photobleaching. The initial decrease may be due to a redistribution of the Alexa647 from the more viscous interior to the exterior of the polymer coat, lowering the quantum yield due to facilitation of bond rotation. ${ }^{31}$ The slight reversible steps in Alexa647 emission were independent of the state of the PC since they were observed when irradiating repeatedly at any given wavelength.

The QD did not exhibit photobleaching but some photobrightening was observed as in previous preparations (compensated in Figure 13 by normalization). The photomodulation of QD fluorescence was robust over the 15 measured cycles, in which the ER changed by $87 \pm 4 \%$ (Figure 13B). Neither fatigue due to the photodegradation of the PC nor spontaneous thermal reversal of cPC was evident at room temperature. Thus, the state of the dual-color psQD was controlled solely via the selected mode of irradiation.
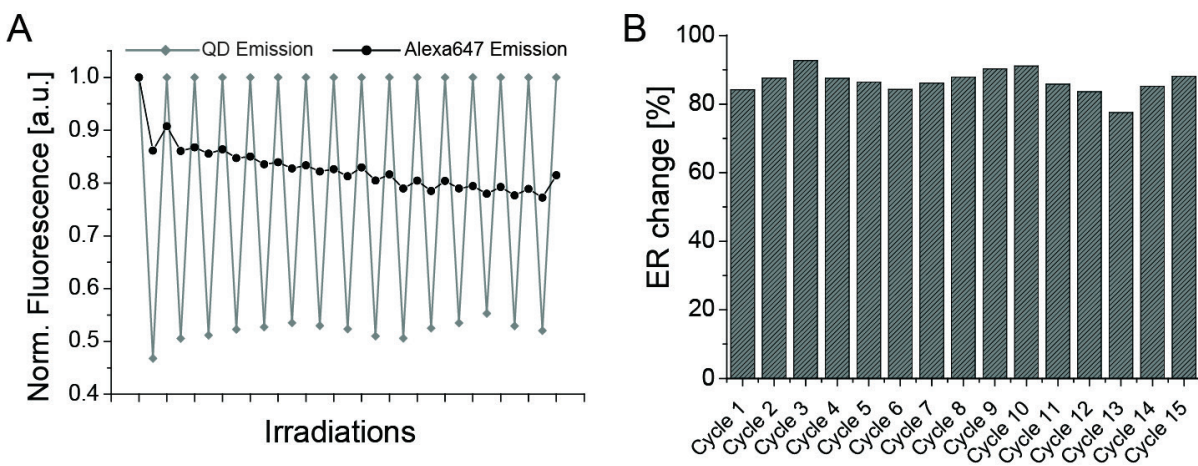

Figure 13: Spectroscopic monitoring of dual-color psQD cycled through open-closed states. Irradiation was for $180 \mathrm{~s} ; 340\left(1.1 \mathrm{~mW} \mathrm{~cm}{ }^{-2}\right)$ and $545\left(6.2 \mathrm{~mW} \mathrm{~cm}^{-2}\right)$. Temperature, $20{ }^{\circ} \mathrm{C}$. (A) Emission of QD after each irradiation, with excitation at $400 \mathrm{~nm}$ and detection at $550 \mathrm{~nm}$. Emission of Alexa647 after each irradiation, with excitation at $600 \mathrm{~nm}$ and detection at $666 \mathrm{~nm}$. (B) Change in the ER during each cycle upon transition from the open to the PS state. ${ }^{18}$ 


\subsubsection{Dual-color psQD Kinetics}

The dual population model of Section 3.2 represented the data well. The dual-color psQD, compared to the first-generation $\mathrm{psQD}$, had much fewer photochromic groups in total, due to the efficient coating, but the FRET parameters $\gamma_{1,2}$ were 4 -fold and 8 -fold larger. The greater FRET efficiency resulted in a higher maximum quenching efficiency ( $50 \%$ vs $40 \%)$. The larger value of $\gamma_{1}$ in the dual psQD probably reflected a reduction in $\mathrm{r}_{\mathrm{DA}}$ of the Class $1 \mathrm{cPC}$, which due to the added linker were placed up to $8.5 \AA$ closer to the QD surface than in the first generation psQD. The $\mathrm{R}_{0}$ computed for the QD-cPC pair was $4.1 \mathrm{~nm}$ in both systems, such that if the differences in $\gamma_{1}(6.6$ vs. 1.5) is ascribed to positional effects alone, Class 1 acceptors would be located $8.3 \AA$ closer to the donor in the PCahx system, in excellent agreement with the stereochemical linker length calculation. The linker may also result in better packing and orientation of the Class 2 PC within the surface ligand-polymer matrix, accounting for the parallel reduction in $\gamma_{2}$.

Table 4: Analysis of the UV-induced photoconversion and QD quenching kinetics

\begin{tabular}{|c|c|c|c|c|c|c|c|c|}
\hline $\begin{array}{c}\text { Dual-color } \\
\text { psQD }\end{array}$ & & $Q_{o c}$ & $Q_{c o, u v}$ & $Q_{c o, v i s}$ & $n_{1}$ & $n_{2}$ & $\gamma_{1}$ & $\gamma_{2}$ \\
\hline & & 0.44 & 0.14 & 0.015 & 3 & 32 & 6.6 & 0.08 \\
\hline \multirow[t]{2}{*}{$\begin{array}{c}\text { Dual-color } \\
\text { ps } Q D\end{array}$} & $k_{\mathrm{o}}$ & $\begin{array}{ll}k_{\mathrm{c}} & k_{\mathrm{QD}} \\
s^{-1} & \end{array}$ & $E_{1, \mathrm{PS}}$ & $E_{2, \mathrm{PS}}$ & $\alpha_{1, \mathrm{PS}}$ & $\alpha_{2, \mathrm{PS}}$ & $\left(\frac{r_{\mathrm{DA}}}{R_{\mathrm{o}}}\right)_{1}$ & $\left(\frac{r_{\mathrm{DA}}}{R_{0}}\right)_{2}$ \\
\hline & 0.01 & $\begin{array}{ll}0.08 & 2.2\end{array}$ & 0.26 & 0.24 & 0.02 & 0.20 & 0.7 & 1.5 \\
\hline
\end{tabular}

Dual-color psQD: CdSe/CdS/ZnS QD (CAN GmbH, Hamburg) coated with PMA 7PCahx 75C12 0.3Al.

For the dual-color psQD system I obtained $\mathrm{r}_{\mathrm{DA}}=3.0 \mathrm{~nm}$ for Class 1 and $6.2 \mathrm{~nm}$ for Class 2, while $\Delta r_{1}=-1.45 \mathrm{~nm}$ and $\Delta r_{2}=1.8 \mathrm{~nm}\left(\mathrm{R}_{\mathrm{o}}=4.1 \mathrm{~nm}\right.$ and $\left.r_{\mathrm{QD}}=3.0 \mathrm{~nm}\right)$. A negative $\Delta r_{1}$ value is physically untenable, demonstrating that the calculated values must be considered within the wider context of the system. Invoking two classes of acceptors, varying in FRET properties, satisfactorily fit the observed data of the psQD systems but there are a number of assumptions that can affect the numerical values obtained.
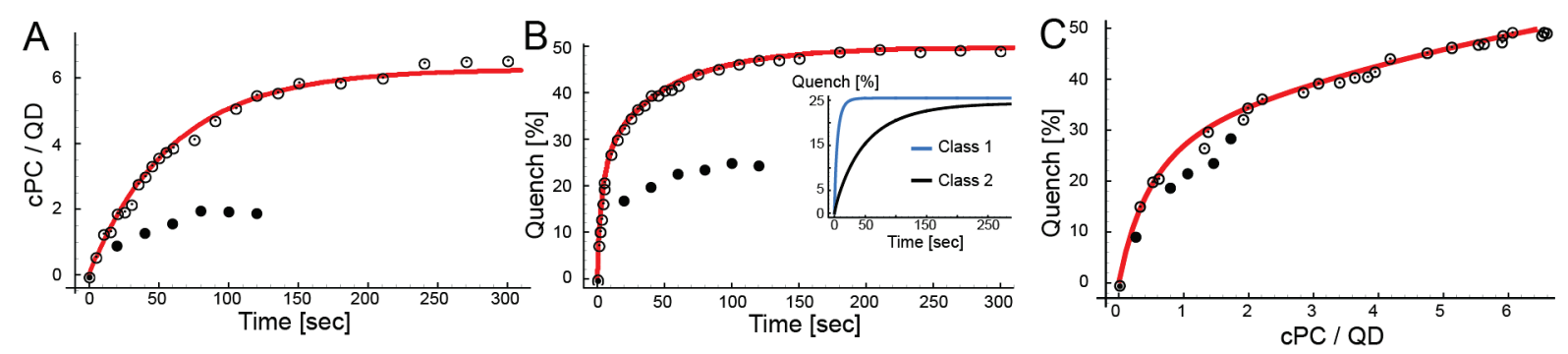

Figure 14: Photoconversion data and fits. Dual-color psQDs were irradiated with $340 \pm 10 \mathrm{~nm}$ UV light,

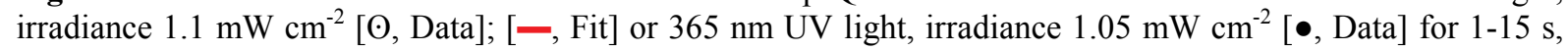
absorbance and fluorescence spectra were recorded, and then a new pulse was applied. (A) Mean value of cPC molecules per QD as a function of irradiation time. (B) Fluorescence quenching of QD as a function of irradiation time. Inset: Quench\% corresponding to the two classes of acceptor as a function of time: - , Class 1; -, Class 2 (C) Fluorescence quenching of QD as a function of mean value of cPC molecules per QD. 
Photoconversion using $365 \mathrm{~nm}$ filters, a more accessible wavelength for fluorescence microscopy, was also realized and can be observed in Figure 14. Due to the less favorable oPC/cPC absorption cross-section ratio, the photostationary state was displaced to lower values of $\alpha_{\mathrm{PS}}$, and QD quenching diminished in accordance with the universal relation between quenching and cPC/QD content, established by exposure to $340 \mathrm{~nm}{ }^{7}$ In Figure $14 \mathrm{C}$ it can be observed that the data-points for both the $340 \mathrm{~nm}$ and $365 \mathrm{~nm}$ irradiations nearly overlap.

\subsubsection{Using Dual-Color psQDs to Study Polymer Coating Stability}

The use of amphiphilic coated QDs as cellular probes has been demonstrated repeatedly, ${ }^{32,33}$ yet if the QDs are to be targeted through polymer modifications it is of interest to know how stable the polymer coating truly is. Storage in buffer (for $>1$ year at $4{ }^{\circ} \mathrm{C}$ ) did not change the psQD properties. The potential influence of cellular interactions, such as passage across the cellular membrane, was one important issue to consider. That is, would cellular uptake strip the polymer from the QD? A collaborative project with J. Azcárate (INIFTA, La Plata, Argentina) was undertaken using polymers containing polarity sensing ESIPT (excited state intramolecular proton transfer) dyes ${ }^{34}$ coated onto gold NPs. The general architecture of these probes, NP coated with PMA based polymer containing a hydrophobic dye, is similar to the psQDs. The NPs were suspended in an aqueous buffer and were titrated with different solvents to observe whether nanoconstructs could act as improved polarity sensors. The titration with $i$-PrOH consistently produced unexpected results. In Figure 15A the N/T band ratio, a measure of environmental polarity, of an $i-\mathrm{PrOH}$ titration of gold particles coated with a polymer containing the ESIPT dye 2-(furan-2-yl)-3-hydroxy-4H-chromen-4-one ${ }^{35}$ is shown. At 30 and $35 \mathrm{i}-\mathrm{PrOH} \%$ discontinuous jumps of the ratio are observed while other solvents (e.g. THF or ACN) present continuous increases. We hypothesized that $i-\mathrm{PrOH}$ was intercalating between the NP and the polymer and at a high enough concentration was stripping the polymer from the surface.

The dual-color psQD provided an excellent opportunity for studying this hypothesis due to the FRET system being so dependent on the acceptor-donor distance. A dual-color psQD solution was titrated with upto $50 \% i$-PrOH. The psQDs had been previously irradiated so as to be in the PS state, meaning maximum QD quench. Controls were carried out by titrating with water and $\mathrm{CHCl}_{3}$ and the same titrations were realized on PMA75C12 coated QDs. The $\mathrm{CHCl}_{3}$ did not interact with the polymer coated QDs, the high negative surface charge density causing the QDs to remain in the aqueous fraction. Dilution with water did not change the relative fluorescence intensity. Thus, the addition of these two solvents did not affect the polymer/QD interaction. 

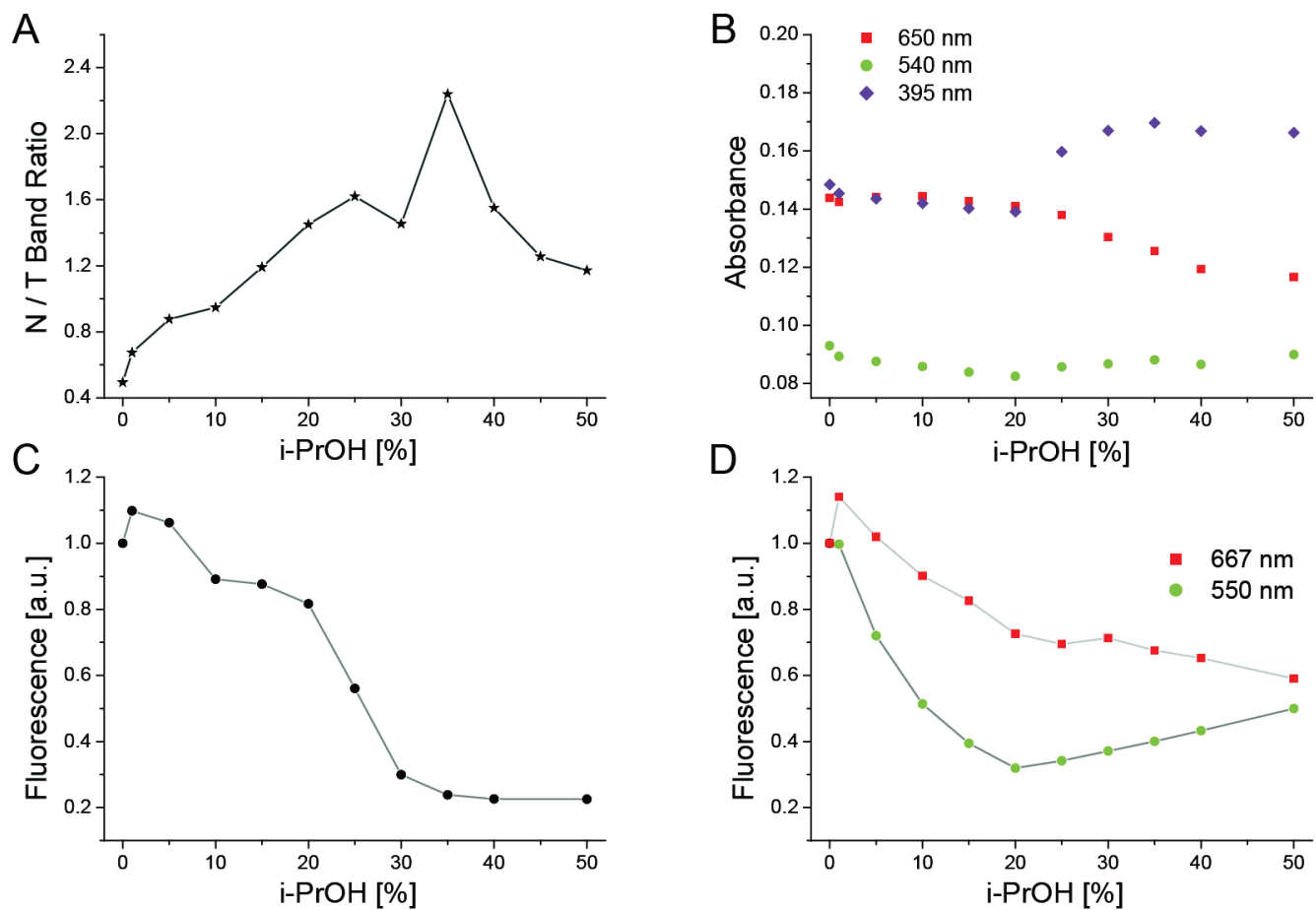

Figure 15: A) N/T band ratio of an Au NP coated with polymers containing polarity sensing dyes as a function of $\mathrm{i}-\mathrm{PrOH} \%$. Observe the sudden changes at 25-35\% i-PrOH. B) Absorbance of dual-color psQD as a function of $i$-PrOH \% C) Emission from polymer coated QD [550 nm] vs. $i$-PrOH \% D) Emission from dual-color psQD $[550 / 667 \mathrm{~nm}]$ as a function of $i-\operatorname{PrOH} \%$.

A cuvette with the sample was titrated with $i$-PrOH while absorbance and steady-state fluorescence was measured (dilution effects were corrected for). As can be seen in Figure 15 the $i$ PrOH titration affects the psQD stability. The control QDs showed that the QY of emission decreased slowly up to $20 \% i$-PrOH then abruptly dropped at $30 \%$ after which it plateaued. The psQD absorbance spectra were constant until 20\% $i-\mathrm{PrOH}$ at which point the $395 \mathrm{~nm}$ absorbance increased and the Alexa647 absorbance decreased. Differing from the control QDs the QY of the dual-color psQD decreased rapidly from initial $i-\mathrm{PrOH}$ addition to $20 \% i-\mathrm{PrOH}$, but then increased. The Alexa647 emission decreases slowly throughout the titration.

The data was interpreted as follows. The initial addition of $i$-PrOH intercalates in between the polymer and QD causing a more polar environment and decreasing the QD QY. FRET is unaffected (or any effect is masked by the change in QY) as the actual QD-cPC distance is not modified. It was noted that the psQD fluorescence decreased faster than the control QD, another experimental result supporting the theory of decreased coating efficiency of polymers with voluminous dyes. At $25 \% i$ $\mathrm{PrOH}$ the polymer begins to detach from the QD and form polymersomes. At this point the QD-cPC distance was modified, and if the QD QY was stable, the FRET decrease leads to the QD emission increase. The absorbance spectra were consistent with this interpretation; the increase in $395 \mathrm{~nm}$ absorbance at $20 \%$ can be attributed to an additional scattering contribution. This same scattering component can be observed clearly in the individual fluorescence spectra. 
This initial study demonstrated the ability of solvents to separate the coating from the NP, and although cell membranes are quite different from $i-\mathrm{PrOH}$ further studies must be realized to determine the fates of the polymer coated NPs when used in a cellular context.

\section{4 psQDs Prepared with Photochromic Polymers Conjugated with Different Linker Length PCs}

A number of psQDs were prepared with the hope of improving the final quenching of the QD emission. These psQDs were all prepared with the same QD (Series A CSS $540 \mathrm{~nm}, \mathrm{CAN}$ GmbH, Hamburg) as the dual-color psQDs but with varying polymers. The polymers varied in PC concentration as well as in the linker length of the PC. Polymers containing over 10\% PC were unable to coat QDs efficiently and could not be studied.

Though the QD quenching never exceeded that obtained from the dual-color psQD, ${ }^{18}$ which presented a 6 carbon linker on the PC, an interesting result was observed. I expected that as the linkers increased in length the $\mathrm{r}_{\mathrm{DA}}$ would decrease and more efficient FRET would lead to higher quenching values, yet it appears as if the 50\% quenching is a photophysical limitation of the QD-PC pcFRET pair and chosen irradiation conditions. The photoconversion conditions present an excess of photons which are absorbed by the QD instead of the oPC and therefore as the individual FRET pair efficiency increases the amount of acceptors in the system will invariably decrease. The $35-50 \%$ quenching is apparently the equilibrium point for the selected $340 \mathrm{~nm}$ continuous wave irradiation conditions. Table 5 characterizes the prepared psQDs.

Table 5: Analysis of the UV-induced photoconversion and QD quenching kinetics

\begin{tabular}{llclccc}
\hline Sample & PC used & $\begin{array}{c}\text { Linker } \\
\text { length }\end{array}$ & Polymer composition & $\begin{array}{c}\text { Max } \\
\text { Quench }\end{array}$ & $\begin{array}{c}\text { cPC/QD at } \\
\text { PS state }\end{array}$ & $k_{o c}^{\prime}\left[\mathrm{s}^{-1}\right]$ \\
\hline 1 & $\mathrm{PCmNH}_{2}$ & 0 & PMA 6PC 70C12 & $37 \%$ & 17.2 & $55 \pm 2$ \\
2 & $\mathrm{PCalaNH}_{2}$ & 2 & PMA 6PCala 75C12 & $35 \%$ & 14.2 & $39 \pm 2$ \\
3 & $\mathrm{PCahxNH}_{2}$ & 6 & PMA 7PCahx 75C12 & $50 \%$ & 6.5 & $75 \pm 3$ \\
4 & $\mathrm{PCaocNH}_{2}$ & 8 & PMA 8PCaoc 75C12 & $38 \%$ & 2.5 & $53 \pm 4$ \\
5 & $\mathrm{PCadoNH}_{2}$ & 12 & PMA 4.5PCado 75C12 & $39 \%$ & 4.2 & $61 \pm 9$ \\
\hline
\end{tabular}




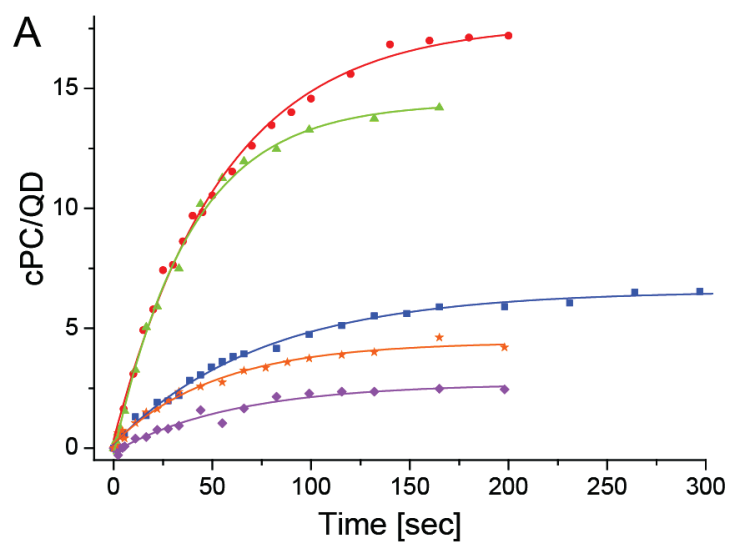

- $\mathrm{PCmNH}_{2}$

$\triangle$ PCalaNH $_{2}$

- $\mathrm{PCahxNH}_{2}$

- $\mathrm{PCaOCNH}_{2}$

$\star \mathrm{PCadoNH}_{2}$
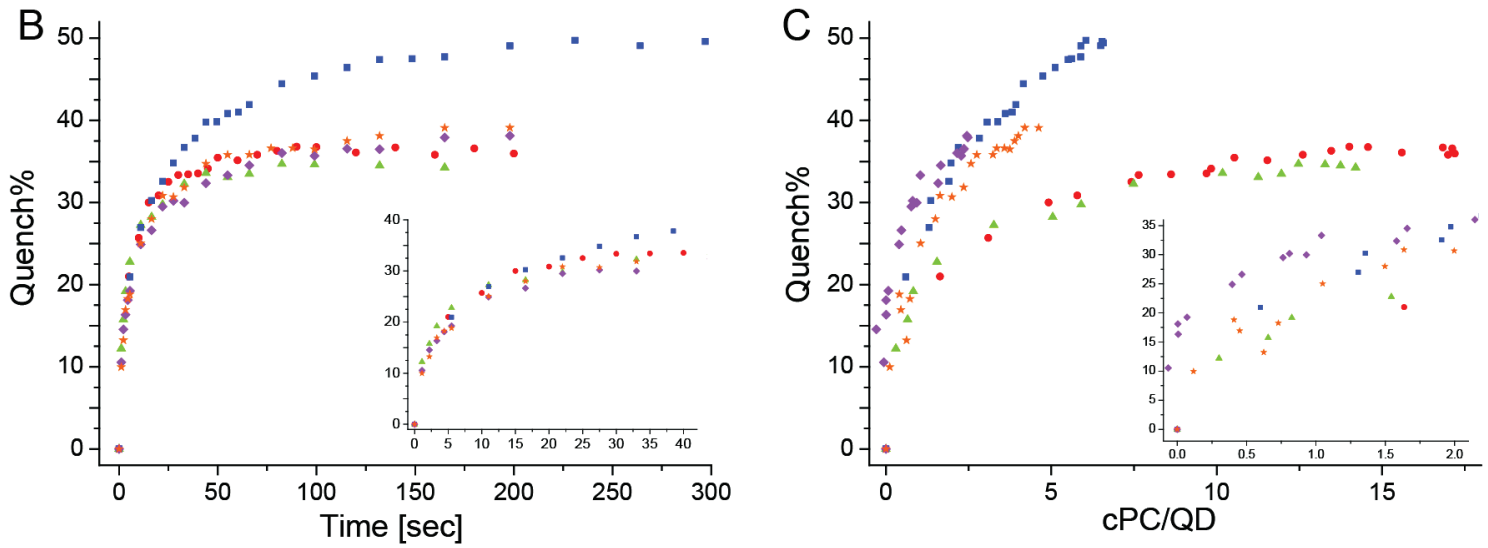

Figure 15: Photoconversion and quenching data. A) Graph of the evolution of cPC/QD as a function of UV (1.0 $\mathrm{mW} \mathrm{cm}$ ) irradiation time. Fit is monoexponential. B) Quenching of QD fluorescence as a function of UV (1.0 $\mathrm{mW} \mathrm{cm}^{-2}$ ) irradiation time. C) Quenching of QD fluorescence as a function of cPC/QD.

Observing Table 5 it can be seen that the $k_{o c}^{\prime}$ does not vary much throught the series of psQDs. The velocity of conversion was similar in all psQDs, but the extent of conversion changed drastically. As the linker length increases the cPC should approach ever closer to the surface of the QD and therefore FRET should increase. From 0-8 carbon linker length this would seem to be the case inasmuch as the content of $\mathrm{cPC} / \mathrm{QD}$ decreases even though the polymers had similar PC concentrations. Once a linker reaches a certain length the packing efficiency might force it away from the QD explaining the uptick in the PCado sample. One would expect a correlation between the velocity and extent of photoconversion, where $\mathrm{PCaoc}$ would be the slowest and $\mathrm{PCmNH}_{2}$ the fastest, but in fact no correlation was found. If we examine the quenching kinetics (Figure 15B) some very interesting results are observed. The QD quench as the psQDs are irradiated with UV light was indistinguishable for the first 30 seconds in all the samples. In fact all the samples, except PCahx, followed the same time course. It was only PCahx which exhibited an increase in the quenching of the QD. It is striking that this occurred since the differences in cPC groups of each sample varied tremendously over that time as seen in Panel A. The data in Figure 15C seems to present 2 subgroups of psQD, a longer linker (6-12) efficient system and a shorter linker (0-2) group which was less efficient. Yet again, for the first $1.5 \mathrm{cPC} / \mathrm{QD}$ all 5 curves are superimposed, and only then separate into the two sub-groups. Though the final quenching was similar for 4 out 5 psQDs the short linkers required a much greater number of cPC. 
In summary, it appears that linker length has a limited utility in modifying the psQD photomodulation. Consistent with the proposed pcFRET model in Section 3.2, the UV light used to photocyclize the PC to the $\mathrm{cPC}$ form excites the QD which transfers energy to any pre-existing $\mathrm{cPC}$ acceptors and forces them back to oPC conformations. The photocyclization constants $\left(k_{o}, Q_{o c, u v}\right)$ are unaffected by FRET but the cycloreversion pathway is highly dependent on the FRET efficiency as seen in Equation 4. As the FRET increases the QD will emit less photons, higher quench, but will also limit the amount of PC that remains in the cPC form. I hypothesize that the quenching limitation is independent of the D-A distance and the total number of PCs placed around the QD (as long as the QD absorbance is greater than the total oPC absorbance at the irradiation wavelength). An interesting experiment would be to wrap the QDs with a previously photoconverted polymers so that they all have $\alpha_{\mathrm{PS}}=0.22$. The $\mathrm{psQDs}$ with the smaller $\mathrm{r}_{\mathrm{DA}}$ should have higher quenching initially but any subsequent cycles would revert to the $<50 \%$ limit. The experiment is problematical due to the fact that the complete psQD preparation up until the first measurement must be realized in the dark. The utilization of different irradiation wavelengths to photocyclize the psQDs may also provide insight. Looking back at the pcFRET model, the rate constants $\left(k_{\mathrm{o}}, k_{\mathrm{c}}\right.$, and $\left.k_{\mathrm{QD}}\right)$ are modified but can be calculated for different wavelengths, multiple variables are wavelength independent $\left(Q_{\mathrm{co}, \mathrm{vis}}, \gamma_{1}, \gamma_{2}, n_{1}\right.$ and $\left.n_{2}\right)$ and the UV quantum efficiency values ( $Q_{\text {oc,uv }}, Q_{\text {co,uv }}$ ) would not be expected to vary considerably. The pcFRET model should therefore have predictive capabilities for the $\alpha_{\mathrm{PS}}$ and maximum quenching at different wavelengths.

Though further investigation in the $n=3-5$ carbon linker length should be undertaken for the moment the PCahx can be regarded as the most advantageous position for the $\mathrm{cPC}$ quencher.

\subsection{Conclusions}

The preparation of psQDs is a simple straightforward process based on coating a QD with an amphiphilic photochromic polymer that provides solubility in aqueous media. The polymers take advantage of a previously underutilized microenvironment, the hydrophobic region between the QD surface and the hydrophilic exterior of the polymer coat, to obtain efficient photoswitching of the PC. The psQD retains all the desirable properties of the original QD (broad excitation, narrow emission, photostability) and allows the brightness of the emission to be modified by light. The psQDs can be further modified by introduction of a second dye, creating an internal standard and allowing for ratiometric imaging capabilities.

A new photophysical model was proposed to account for the observed quenching data. The model is applicable to psQDs independently of the linker PC chosen for polymer conjugation. A very advantageous feature of the psQD system is the prominent quenching of the QD exerted by low degrees of photoconversion, a characteristic of all the presented samples. This is a promising property for obtaining modulation using short light pulses in a fluorescence microscopy setting. 
By varying the QD-PC distance it was observed that there appeared to be a limit to the attainable degree of QD quenching with the chosen PC. Placing the PC closer to the QD so as to increase the FRET efficiency was accompanied by a lower number of acceptors. A different diheteroarylethene might allow a higher $\alpha_{\mathrm{PS}}$, and therefore greater quenching. The recent development of fluorescent diheteroarylethenes may provide a new strategy for creating dual-color switchable QDs where the FRET acceptor now presents its own fluorescence. ${ }^{36}$

\section{References}

1. Walling, M. A.; Novak, J. A.; Shepard, J. R. Quantum Dots for Live Cell and in Vivo Imaging. Int J Mol Sci 2009, 10, 441-491.

2. Ishikawa-Ankerhold, H. C.; Ankerhold, R.; Drummen, G. P. C. Advanced Fluorescence Microscopy Techniques-FRAP, FLIP, FLAP, FRET and FLIM. Molecules 2012, 17, 4047-4132.

3. Levitt, J. A.; Matthews, D. R.; Ameer-Beg, S. M.; Suhling, K. Fluorescence Lifetime and PolarizationResolved Imaging in Cell Biology. Curr Opin Biotechnol 2009, 20, 28-36.

4. Bates, M.; Huang, B.; Zhuang, X. Super-Resolution Microscopy by Nanoscale Localization of PhotoSwitchable Fluorescent Probes. Curr Opin Chem Biol 2008, 12, 505-514.

5. Dedecker, P.; Mo, G. C.; Dertinger, T.; Zhang, J. Widely Accessible Method for Superresolution Fluorescence Imaging of Living Systems. Proc. Natl. Acad. Sci. U.S.A. 2012, 109, 10909-10914.

6. Chudakov, D. M.; Matz, M. V.; Lukyanov, S.; Lukyanov, K. A. Fluorescent Proteins and Their Applications in Imaging Living Cells and Tissues. Physiol Rev 2010, 90, 1103-1163.

7. Giordano, L.; Jovin, T. M.; Irie, M.; Jares-Erijman, E. A. Diheteroarylethenes as Thermally Stable Photoswitchable Acceptors in Photochromic Fluorescence Resonance Energy Transfer (pcFRET). J. Am. Chem. Soc. 2002, 124, 7481-7489.

8. Jares-Erijman, E.; Giordano, L.; Spagnuolo, C.; Lidke, K.; Jovin, T. Imaging Quantum Dots Switched on and Off by Photochromic Fluorescence Resonance Energy Transfer (pcFRET). Mol. Cryst. Liq. Cryst. 2005, 430, 257-265.

9. Saitoh, M.; Fukaminato, T.; Irie, M. Photochromism of a Diarylethene Derivative in Aqueous Solution Capping with a Water-Soluble Nano-Cavitand. J. Photochem. Photobiol. A-Chem. 2009, 207, 28-31.

10. Medintz, I. L.; Trammell, S. A.; Mattoussi, H.; Mauro, J. M. Reversible Modulation of Quantum Dot Photoluminescence Using a Protein- Bound Photochromic Fluorescence Resonance Energy Transfer Acceptor. J. Am. Chem. Soc. 2004, 126, 30-31.

11. Erno, Z.; Yildiz, I.; Gorodetsky, B.; Raymo, F. M.; Branda, N. R. Optical Control of Quantum Dot Luminescence Via Photoisomerization of a Surface-Coordinated, Cationic Dithienylethene. Photochem Photobiol Sci 2010, 9, 249-253.

12. Hezinger, A. F.; Tessmar, J.; Gopferich, A. Polymer Coating of Quantum Dots---a Powerful Tool toward Diagnostics and Sensorics. Eur J Pharm Biopharm 2008, 68, 138-152.

13. Janczewski, D.; Tomczak, N.; Han, M.-Y.; Vancso, G. J. Synthesis of Functionalized Amphiphilic Polymers for Coating Quantum Dots. Nat. Protoc. 2011, 6, 1546-1553.

14. Díaz, S. A.; Menendez, G. O.; Etchehon, M. H.; Giordano, L.; Jovin, T. M.; Jares-Erijman, E. A. Photoswitchable Water-Soluble Quantum Dots: pcFRET Based on Amphiphilic Photochromic Polymer Coating. ACS Nano 2011, 5, 2795-2805. 
15. Sillen, A.; Engelborghs, Y. The Correct Use of "Average" Fluorescence Parameters. Photochem. Photobiol. 1998, 67, 475-486.

16. Fernandez-Arguelles, M. T.; Yakovlev, A.; Sperling, R. A.; Luccardini, C.; Gaillard, S.; Medel, A. S.; Mallet, J. M.; Brochon, J. C.; Feltz, A.; Oheim, M.; Parak, W. J. Synthesis and Characterization of Polymer-Coated Quantum Dots with Integrated Acceptor Dyes as FRET-Based Nanoprobes. Nano Lett 2007, 7, 2613-2617.

17. Sperling, R. A.; Liedl, T.; Duhr, S.; Kudera, S.; Zanella, M.; Lin, C. A. J.; Chang, W. H.; Braun, D.; Parak, W. J. Size Determination of (Bio)Conjugated Water-Soluble Colloidal Nanoparticles: A Comparison of Different Techniques. J. Phys. Chem. C 2007, 111, 11552-11559.

18. Díaz, S. A.; Giordano, L.; Jovin, T. M.; Jares-Erijman, E. A. Modulation of a Photoswitchable DualColor Quantum Dot Containing a Photochromic FRET Acceptor and an Internal Standard. Nano Lett. 2012, 12, 3537-3544.

19. Jares-Erijman, E. A.; Jovin, T. M. FRET Imaging. Nat. Biotechnol. 2003, 21, 1387-1395.

20. Derfus, A. M.; Chan, W. C. W.; Bhatia, S. N. Probing the Cytotoxicity of Semiconductor Quantum Dots. Nano Lett. 2004, 4, 11-18.

21. Carrillo-Carrion, C.; Cardenas, S.; Simonet, B. M.; Valcarcel, M. Quantum Dots Luminescence Enhancement Due to Illumination with UV/Vis Light. Chem. Commun. 2009, 5214-5226.

22. Gómez, D. E.; Califano, M.; Mulvaney, P. Optical Properties of Single Semiconductor Nanocrystals. Phys. Chem. Chem. Phys. 2006, 8, 4989.

23. Métivier, R.; Badré, S.; Méallet-Renault, R.; Yu, P.; Pansu, R. B.; Nakatani, K. Fluorescence Photoswitching in Polymer Matrix- Mutual Influence between Photochromic and Fluorescent Molecules by Energy Transfer Processes. J. Phys. Chem. C 2009, 113, 11916-11926.

24. Celej, M. S.; Jares-Erijman, E. A.; Jovin, T. M. Fluorescent N-Arylaminonaphthalene Sulfonate Probes for Amyloid Aggregation of Alpha-Synuclein. Biophys. J. 2008, 94, 4867-4879.

25. Murakami, M.; Miyasaka, H.; Okada, T.; Kobatake, S.; Irie, M. Dynamics and Mechanisms of the Multiphoton Gated Photochromic Reaction of Diarylethene Derivatives. J. Am. Chem. Soc. 2004, 126, 14764-14772.

26. Yildiz, I.; Deniz, E.; Raymo, F. M. Fluorescence Modulation with Photochromic Switches in Nanostructured Constructs. Chem Soc Rev 2009, 38, 1859-1867.

27. Schreiber, F. Structure and Growth of Self-Assembling Monolayers. Prog. Surf. Sci. 2000, 65, 151-256.

28. Grecco, H. E.; Lidke, K. A.; Heintzmann, R.; Lidke, D. S.; Spagnuolo, C.; Martinez, O. E.; JaresErijman, E. A.; Jovin, T. M. Ensemble and Single Particle Photophysical Properties (Two-Photon Excitation, Anisotropy, FRET, Lifetime, Spectral Conversion) of Commercial Quantum Dots in Solution and in Live Cells. Microsc Res Tech 2004, 65, 169-179.

29. Parak, W. J.; Pellegrino, T.; Plank, C. Labelling of Cells with Quantum Dots. Nanotechnology 2005, 16, R9-R25.

30. Schlegel, G.; Bohnenberger, J.; Potapova, I.; Mews, A. Fluorescence Decay Time of Single Semiconductor Nanocrystals. Phys. Rev. Lett. 2002, 88, 137401.

31. Levitus, M.; Ranjit, S. Cyanine Dyes in Biophysical Research: The Photophysics of Polymethine Fluorescent Dyes in Biomolecular Environments. Q. Rev. Biophys. 2010, 44, 123-151. 
32. Hühn, D.; Kantner, K.; Geidel, C.; Brandholt, S.; De Cock, I.; Soenen, S. J. H.; Rivera_Gil, P.; Montenegro, J.-M.; Braeckmans, K.; Müllen, K.; Nienhaus, G. U.; Klapper, M.; Parak, W. J. PolymerCoated Nanoparticles Interacting with Proteins and Cells: Focusing on the Sign of the Net Charge. ACS Nano 2013, 7, 3253-3263.

33. Tomczak, N.; Janczewski, D.; Dorokhin, D.; Han, M. Y.; Vancso, G. J. Enabling Biomedical Research with Designer Quantum Dots. Methods Mol Biol 2012, 811, 245-265.

34. Klymchenko, A. S.; Demchenko, A. R., Multiparametric Probing of Microenvironment with Solvatochromic Fluorescent Dyes. In Fluorescence Spectroscopy, Brand, L.; Johnson, M. L., Eds. Elsevier Academic Press Inc: San Diego, 2008; Vol. 450, pp 37-58.

35. Yushchenko, D. A.; Fauerbach, J. A.; Thirunavukkuarasu, S.; Jares-Erijman, E. A.; Jovin, T. M. Fluorescent Ratiometric MFC Probe Sensitive to Early Stages of A-Synuclein Aggregation. J. Am. Chem. Soc. 2010, 132, 7860-7861.

36. Gillanders, F.; Giordano, L.; Díaz, S. A.; Jovin, T. M.; Jares-Erijman, E. A. Photoswitchable Fluorescent Diheteroarylethenes. Substituent Effects on Photochromic and Solvatochromic Properties. In Preperation 2013. 


\section{CHAPTER 4: SINGLE PARTICLE MICROSCOPY OF PHOTOSWITCHABLE QUANTUM DOTS}

\section{Introduction}

In the late 1990s room temperature single particle (or molecule) spectroscopy became a widely accessible technique, and it quickly found applications in many different fields. ${ }^{1}$ The development of single particle microscopy (SPM) soon followed, based principally on laser scanning confocal microscopy or TIRF (total internal reflection fluorescence) microscopy. ${ }^{2}$ The advantages provided by SPM include obtaining the probability distribution function of a parameter, independence from time synchronization, and of course the observation of previously unknown processes (QD blinking as a classic example).

A model was presented in Chapter 3 for the photomodulation of psQDs, yet the actual effect of PC photocyclization on individual QDs remains unknown. Does 50\% imply the presence of 50\% dark QDs or the dimming of all psQDs, does it affect the blinking statistics, or change the on/off duty cycles? The possible applications of psQDs depend on the resolution to this uncertainty by SPM analysis. In SPM the underlying distribution and not the ensemble average is evaluated, allowing for the construction of a histogram of experimental values. This distribution provides more information, for example allowing the detection of sub-populations and their characteristics. ${ }^{1}$

An interdisciplinary project was undertaken with the group of Prof. Vinod Subramaniam (Nanobiophysics Chair, Univ. of Twente, Enschede, The Netherlands) to study psQDs at the single particle level. The microscope set-ups were optimized by Niels Zijlstra, the initial psQD film preparation and imaging was realized collectively, all other steps were realized individually. The measurements were realized in two separate research stays in Enschede and are presented below.

\subsection{Imaging psQDs with a Time Correlated Laser Scanning Confocal Microscope}

The intent was to observe the psQDs on a cover slip using area scans and then focus on specific psQDs of interest. This would allow for intensity measurement time traces and fluorescence lifetime determinations. The samples would be photoswitched from the open state to the PS state or vice versa and then any statistical differences noted.

\subsubsection{Sample Preparation}

The initial study consisted of first generation psQDs. ${ }^{3}$ These were the samples presented in Section 3.1. (CdSe/ZnS core-shell QDs coated with PMA 6PC 70C12). The samples presented maximal fluorescence quenching of $41 \%$ and $\sim 30 \%$ modulation of fluorescence lifetime. Controls were realized with QDs coated with PMA 75C12. The sample was prepared on glass coverslips that 
had been previously cleaned by placing them in $65 \%$ nitric acid for over 3 days. The coverslips were rinsed with HPLC water and $\mathrm{MeOH}$ and allowed to dry. The psQDs were diluted to 1-2 $\mathrm{nM}$ in a $2 \%$ (w/v) PVA (polyvinyl alcohol) solution. The solution was spincoated for $10 \mathrm{~s}$ at $6000 \mathrm{rpm}$, resulting in a thin PVA/psQD layer. ${ }^{4}$ The dilution allowed the psQDs to be well separated so that they were beyond the microscopes diffraction limit insuring that each observed particle was only one psQD. Previous TEM images showed that psQDs do not aggregate in buffer solution. ${ }^{3}$

\subsubsection{Experimental Set-up}

Scanning confocal microscope provide very high contrast by sequentially exciting pixels point by point. With the advent of lasers, the excitation beam could now be easily focused into a very small (diffraction limited) focal volume, exciting only the fluorophores found in that small volume. This creates a very sensitive measurement, ideal for the detection of single particles. ${ }^{5}$ The point by point excitation causes the interrogation of large areas to be very slow, an important limitation for moving samples such as live cells. The psQD sample is prepared in a PVA film, fixing the location; therefore the speed limitation was not important for our requirements. In conjunction with the scanning confocal microscope a time correlated single photon counter (TCSPC) is coupled to the detector. The TCSPC determines the arrival time of each photon emitted by the fluorophore, as more photons arrive a count vs. arrival time histogram is built up. The histogram can then be fit with exponential decay functions to determine the lifetime of an individual emitter and as has been previously noted lifetimes are very sensitive to FRET.

The experiments were performed on a custom-built inverted confocal microscope with an epiillumination configuration (illumination and collection are through the same objective). The objective was a $60 \mathrm{x}$ water creating a $5 \times 5 \mu \mathrm{m}$ scan area with a $100 \mathrm{~nm}$ pixel size. The excitation source was a $485 \mathrm{~nm}$ pulsed diode laser with a $20 \mathrm{MHz}$ repetition rate and $750 \mathrm{~W} \mathrm{~cm}^{-2}$ intensity. The emission was spatially filtered using a $50 \mu \mathrm{m}$ pinhole and was subsequently focused onto a single photon avalanche diode connected to a photon counting module (PicoHarp300, Picoquant). ${ }^{4}$ Reflected excitation light was suppressed with a long pass filter. An Hg lamp was used for the photoswitching irradiation, with adequate filters selected: UV (330-385 nm, $\left.12 \mathrm{~mW} \mathrm{~cm}^{-2}\right)$ and Visible $\left(550 \pm 15 \mathrm{~nm}, 31 \mathrm{~mW} \mathrm{~cm}^{-2}\right)$.

\subsubsection{Results and Discussion}

The set-up was first tested with control QDs. After spin coating the samples, scans were realized to assure that the concentration of QDs allowed for sufficient QDs per scan area but that they remained well separated. A single QD will be detected by more than a single pixel due to the Abbe diffraction limit. After a selected area scan was imaged individual psQDs were chosen to realize single particle traces. By focusing the laser on a single spot we could obtain traces of fluorescence emission over time. QD blinking, the random ON-OFF emission states of the QD under uninterrupted excitation, were observed in these time traces as binary on-off states. ${ }^{6}$ QD blinking was evidence, though inconclusive, that a single emitter was being examined. QDs can also bleach, although they are 
much more photostable than traditional organic fluorophores. The fluorescence lifetime information was encoded in the single QD time-traces and could be analyzed as well.
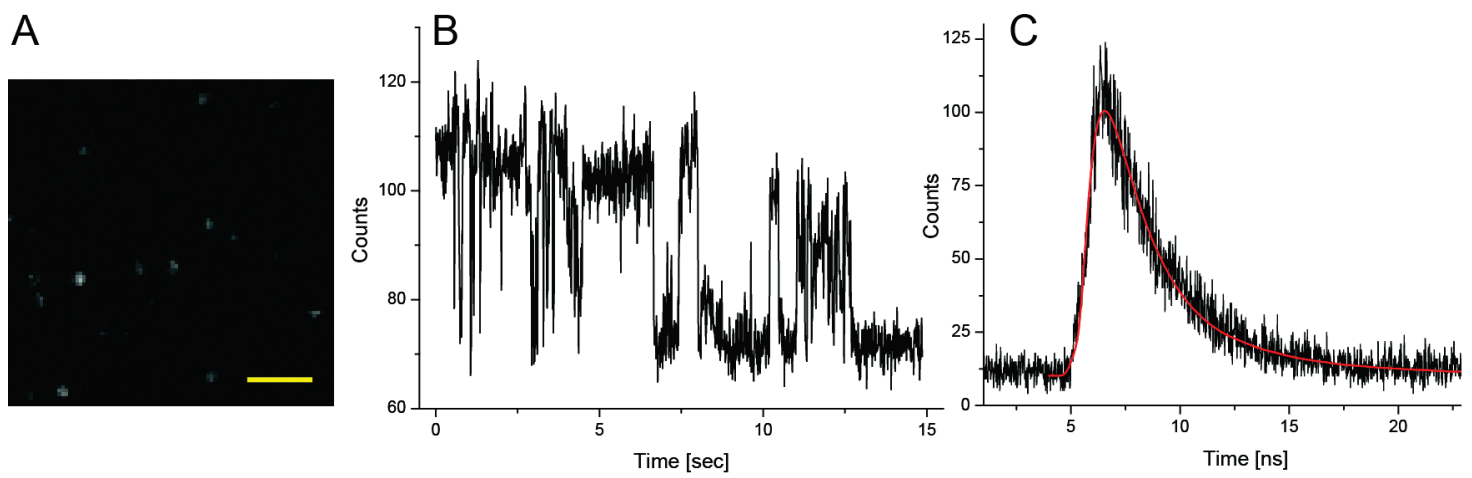

Figure 1: Imaging control QDs. A) Area scan. Scale bar, $1 \mu \mathrm{m}$. B) Time-trace C) Lifetime and corresponding fit.

Scan areas of interest were chosen with at least 5 initial particles that could be designated as psQDs. All samples were in the open state initially, corresponding to the maximum emission of the ensemble population. Areas of interest were defined, irradiated with UV or visible light, and then scanned again (See Figure 2). In most cases the initial irradiation was with visible light so as to guarantee that the psQDs were in the open form. Lateral drift was minimal and when detected it was corrected manually. Z-axis drift was also an issue, requiring manual refocusing. The choice of psQDs for measuring time-traces was arbitrary.

The data analysis was optimized to detect the largest amount of psQDs while avoiding artifacts such as multiple particles being marked as a single psQD. The analysis was then automated through a series of macros in ImageJ (1.44p) for area scans and Excel for time-traces. Specifics can be found in the experimental section; an overview is provided below.

Area Scans:

1. An image scan was imported and cropped to eliminate border effects.

2. Background was subtracted and an image threshold was applied.

3. The Image was transformed into a Binary image and individual QDs selected using optimized parameters. These areas were marked as ROI (regions of interest) and the coordinates were saved.

4. The ROI masks were applied to the image after cropping and background subtraction.

5. Measurements were carried out, providing a list with the mean, max, and minimum intensity of each detected psQD, as well as the measured area.

Time-Traces:

1. Individual time-traces were separated and a threshold applied. A QD was considered ON if the intensity was $>60$ counts and OFF for lower values.

2. An event was defined as the transition of a QD from OFF to ON. The borders of detection $\left(\mathrm{t}_{0}\right.$ and $t_{\text {end }}$ ) were considered as OFF. 
A representative experiment is presented below, in which a psQD/PVA film was irradiated 5 times. The number of psQDs was determined from the individual areas above the threshold.

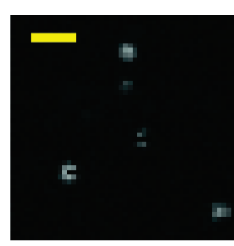

5212

6

73

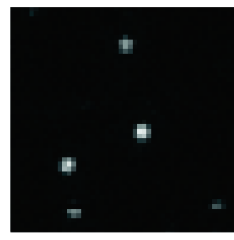

5895

5

92

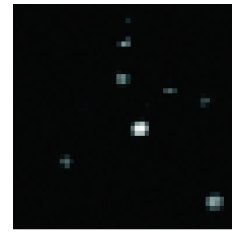

5594

6

87

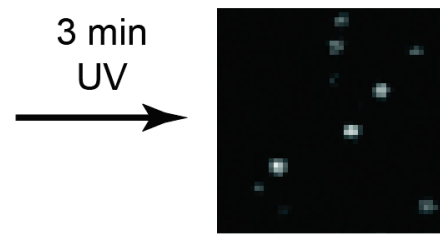

8165

9

80

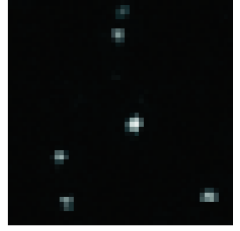

5949

5

90

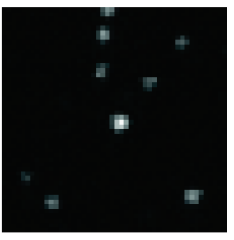

7803

8

81

Figure 2: Area scans of psQDs. Scale bar, $1 \mu \mathrm{m}$.

We expected to observe correlation of the number or intensity of psQDs with their state of photoconversion, i.e. fewer and/or dimmer psQDs in the PS state and brighter and/or more psQDs in the open state. Such a correlation was not observed. After irradiation a field could be much brighter indicative of previously undetected psQDs or the psQDs appeared to be bleached. The data from Figure 2 is discussed, but I reiterate that it is representative of all the experiments. There are only 4 psQDs in Figure 2 that are present throughout the entire experiment, but the number of detected psQDs oscillates between 5 and 9 with a total of 11 different psQDs identified. What is most surprising is the appearance of various new psQDs, in the upper right corner, towards the end of the experiment (Scans 5 and 6). The PVA film should make diffusion of the psQDs impossible, suggesting that the psQDs were always present but where dark through the first series of scans. The fact that this occurs after UV irradiation is what is most puzzling. In a similar manner psQDs shifting to OFF states occurred after visible irradiation. The average intensity of the psQDs does not correlate with the irradiation series either. The UV irradiation can both increase (Scan 3) and decrease (Scan 5) the intensity while the visible irradiation behaved similarly, though not in this specific example. Blinking also occurred, as can be seen in the first image in which individual scan lines within single QDs were dark.

The brightest psQDs in the first scan were selected for time-trace measurements and processing. Traces of given psQDs were measured for 10 seconds with $10 \mathrm{~ms}$ binning, and after each 
irradiation the same psQD was measured again. Figure 1B provides a good representation of the obtained time traces.

A
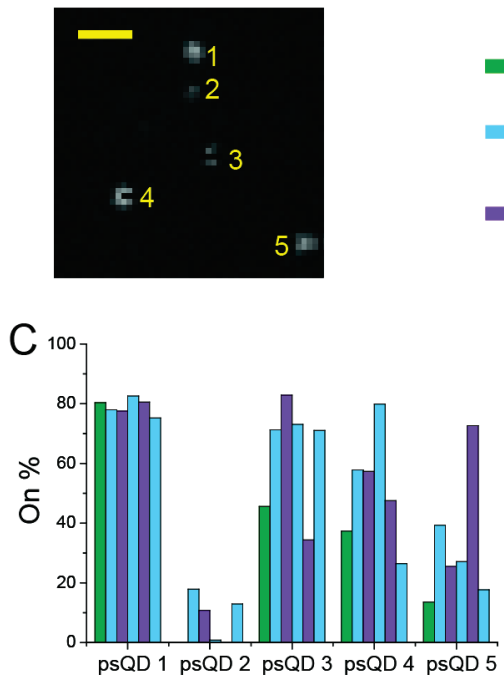

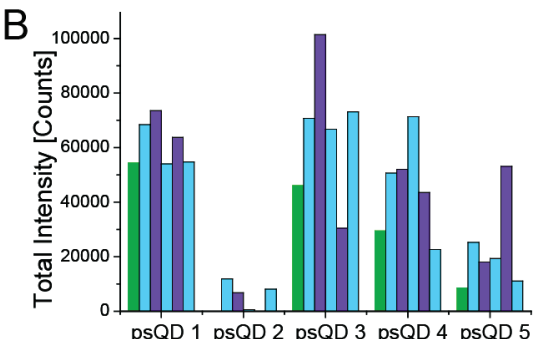

D

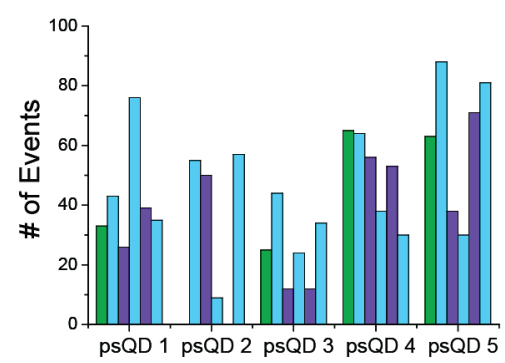

Figure 3: Statistical analysis of the time traces of 5 psQDs throughout a photoswitching experiment. For irradiation times see Figure 2. A) Selected psQDs. B) Total intensity. C) Percentage of time-trace that the psQD was ON. D) Number of blinking events during the time-trace.

Each psQDs was analyzed, but no clear pattern was discernible. For example psQD 1 did not vary much throughout the scans while psQD 3 did, but had both its brightest and darkest state after UV irradiation. Thus, no conclusions could be derived at this time from the photoswitching experiments realized with the scanning confocal instrument.

An unexpected observation can be seen in Figure 4. A few psQDs presented intermediate positions between the ON-OFF states. Initially we attributed this to a FRET effect, but it was observed in both the open and PS states. At this time we cannot explain this phenomenon, though we speculate that it has to do with the polymer coating efficiency and its effect on the fluorescence quantum yield of the psQDs. The acquired lifetime data did not provide any additional insight.

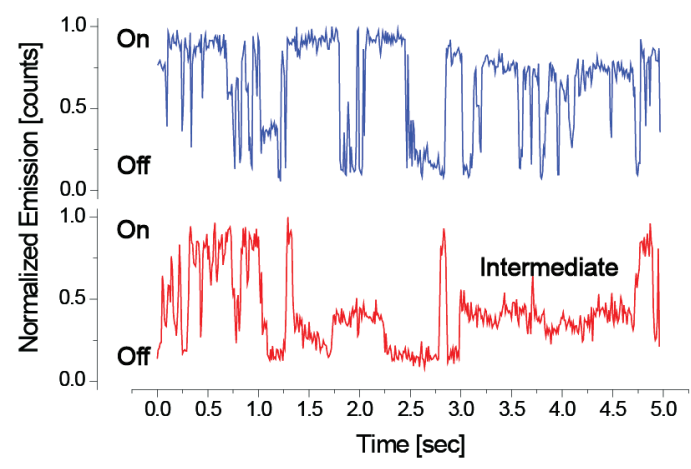

Figure 4: Two examples of psQD time-traces. The upper (blue) example only presents ON-OFF QD blinking, while the lower (red) trace shows intermediate intensity states. 
It became clear that a larger set of measurements was required in order to correctly apply statistical analysis. But before attempting further photoswitching experiments the end points, i.e. the open and PS states, were examined in more detail.

\subsection{Imaging psQDs with Single Particle TIRF Microscopy}

A TIRF microscope allowed the interrogation of a larger field of view which contained more psQDs. The illumination and read-out of the whole field was in real time. The output was in the form of avi movies of the blinking QDs which were then analyzed as individual time-traces.

\subsubsection{Experimental Set-up}

TIRF microscopy presents the advantage of only illuminating a thin region $(<200 \mathrm{~nm})$ in the Z-axis. By using a high incidence angle for the excitation laser all the actual light is reflected due to the differences in the refractive index of the glass and air. At the same time this creates a very thin electromagnetic field (evanescent wave) that serves as the excitation source for the fluorophore. ${ }^{7} \mathrm{~A}$ high signal to noise ratio is achieved, allowing for the detection of the single psQDs, but the detection of fluorophore lifetimes was not possible.

The experiments were performed on a Nikon N-STORM with a Perfect Focus System, which eliminated the need for manual refocusing. We utilized a 100x oil objective and a $488 \mathrm{~nm}$ laser for excitation with $505 \mathrm{~nm}$ bandpass filters and $100 \mathrm{~ms}$ binning for detection. The samples were prepared in similar manner as before (Section 4.1.1) but it was observed that for TIRF illumination the sample was much brighter in the absence of the supporting PVA film. psQDs were thus diluted in pure HPLC water and spin-coated on coverslips.

The imaged psQDs were the dual-color psQDs ${ }^{8}$ presented in Section 3.3 and psQDs prepared with PCaocNH $\mathrm{PH}_{2}(\mathrm{CdSe} / \mathrm{CdS} / \mathrm{ZnS}$ core-shell-shell QD coated with PMA 8PCaoc 75C12). Control QDs (CdSe/CdS/ZnS core-shell-shell QD coated with PMA 75C12) were also imaged in the same conditions. In contrast to the first experiments, each QD sample was split into two fractions. One fraction was irradiated with UV light in solution until it attained the PS state and the other with visible light so as to be in the open state. This provided samples that were identical (composition, concentration, and buffer) except for their photochromic state. The solutions were protected from light. Absorbance and fluorescence spectra acquired before imaging confirmed that the PS had been established and was stable. Once the end states were characterized, we subjected the samples to a photoswitching protocol (see Section 4.2.4).

\subsubsection{Data Analysis}

The raw data consisted of avi movies, duration time $10 \mathrm{~s}$, of the samples in the open and PS state. The movie provides the intensity of each pixel through the duration of the experiment. The movies were processed with macros programmed in ImageJ and Excel. The output was the intensity 
time-trace of every identified QD in the field. Specifics can be found in the experimental section; an overview is provided below.

Movie Processing:

1. Each movie was imported as a stack of images and cropped to eliminate border effects.

2. Background was subtracted from each image.

3. A projection of the maximum intensity of each pixel throughout the stack was created.

4. A threshold was applied to the Max Intensity projection

5. The image created in 4 was analyzed to find all areas representing individual QDs using selected optimized parameters. These areas were designated as ROIs and the coordinates were saved.

6. The ROI masks were applied to the original stack after cropping and background subtraction (Step 2).

7. Each ROI provided a time trace.

8. Individual time-traces were separated and a threshold applied. An appropriate threshold was determined to be 20 counts per bin.

9. An event was defined as the switching of a QD from OFF to ON. The borders of detection $\left(\mathrm{t}_{0}\right.$ and $\left.t_{\text {end }}\right)$ were considered as OFF.
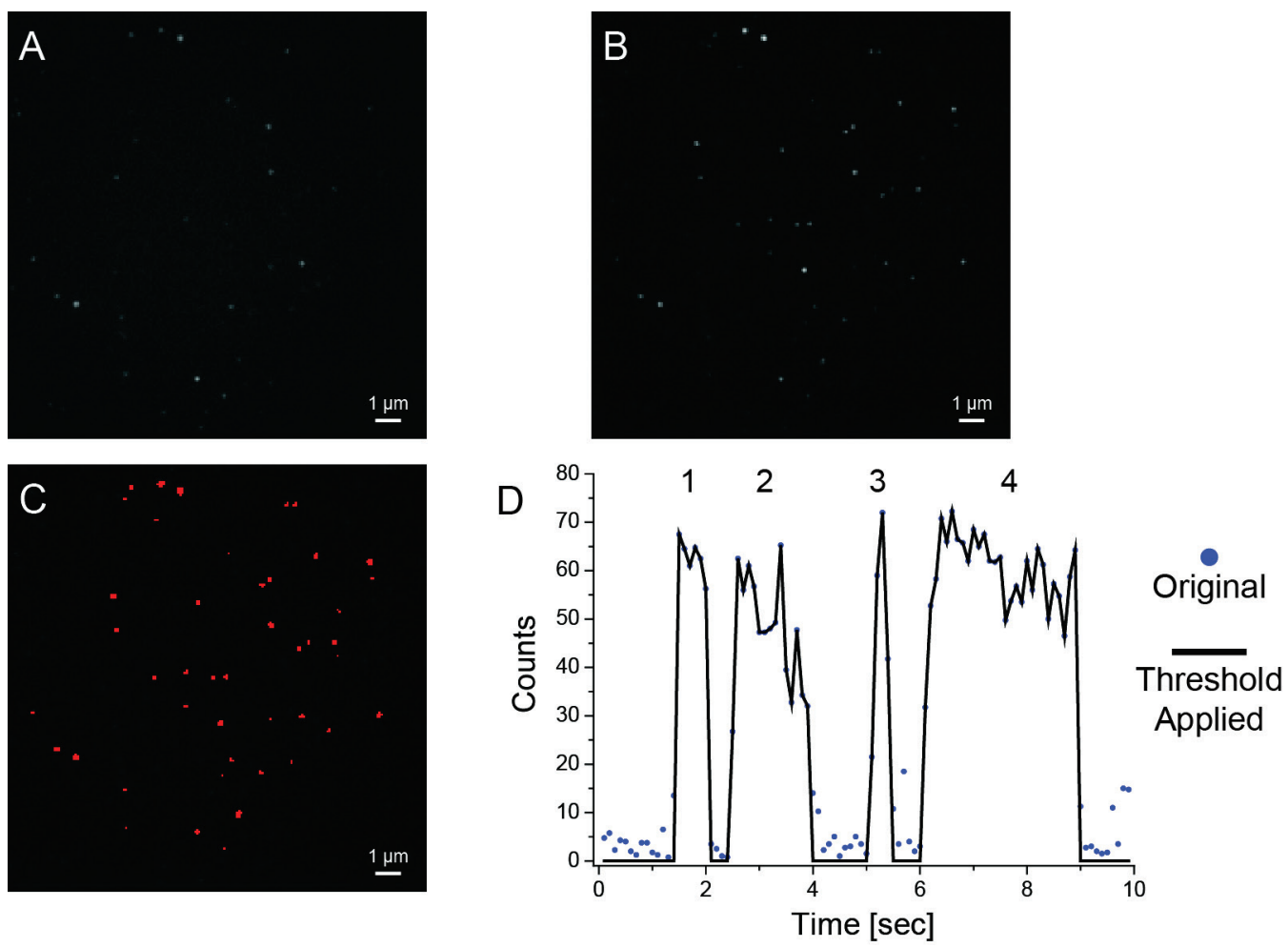

Figure 5: Images corresponding to each analysis step. A) Original Data. B) Max Intensity projection. C) Threshold and ROI analysis. D) Time-trace of an individual psQD. The original data is shown as blue dots, the threshold data used to determine overall intensities and number of events is shown in black.

\subsubsection{Analysis of Open and PS State Measurements}

We wished to correlate the difference in the ensemble intensities of the open and PS state to a parameter that can be observed with the SPM. The analysis provides multiple quantitative values, 
some can be applied to an individual psQD, while some are derived from the entire observed field. The parameters of comparison are defined/measured as follows:

\# of $\mathrm{psQDs}=\mathrm{A}$ particle was considered a psQD when the intensity exceeded the threshold for at least one frame of the movie and when the particle satisfied the size/shape requirements.

$\mathrm{ON} \%=$ The percentage of frames in which the QD intensity was above the set threshold.

Sum $=$ The total intensity (number of counts) throughout the movie. This parameters was determined for single psQDs or to the entire field. OFF bins were considered as having zero intensity.

\# of Events $=$ The number of times a psQD blinked during the movie, as defined in Step 9 (Section 4.2.2)

The number of psQDs imaged was at least 94 in all cases. The results of the two samples are presented below in individual tables and figures. For the PCaoc psQDs (CdSe/CdS/ZnS core-shellshell QD coated with PMA 8PCaoc 75C12) six fields were imaged for the Open and PS state. The results of the analyses are given in Table 1.

Table 1: PCaoc psQDs. Presented uncertainties correspond to the sample standard deviation.

\begin{tabular}{lll}
\hline & Open State & PS State \\
\hline Sum of all Fields [counts x 10 $]$ & 33.3 & 16.3 \\
Mean Sum of Fields [counts x 10 $]$ & $5.5 \pm 1.7$ & $2.7 \pm 0.7$ \\
\# of psQDs & 204 & 94 \\
Mean psQD Intensity [counts] & $28 \pm 15$ & $31 \pm 15$ \\
Mean ON \% & $59 \pm 25$ & $64 \pm 25$ \\
Mean \# of Events & $6.5 \pm 3.5$ & $6.5 \pm 3.5$ \\
\hline
\end{tabular}

It is not reflected in the results tables but the maximum and minimum intensity of most psQDs is well represented by Figure 5D. When a QD was $\mathrm{ON}$ its intensity was 70-80 counts per bin and the background intensity was 0-10 counts for almost every psQD. When individual psQD properties were examined it appears as if there were no differences, the bottom three rows in Table 1 show mean values that are indistinguishable within the given statistical limits. It appears as if the photochromic state of the psQDs did not affect the intensity, blinking statistics, or the ON/OFF ratio. However, the PCaoc psQDs presented a 40\% intensity difference in ensemble solution measurements, and Table 1 indicates there were $>2$ times as many psQDs in the open than in the PS state. We reiterate that the fields were chosen at random and this observation was only noted upon applying the data analysis. In addition, the overall intensity of the illuminated fields decreased by a factor of 2 between the open and PS state, a value exceeding that of the ensemble population. This result may reflect to some degree the exclusion of low intensity QDs in both samples. 
Figure 6 shows the histogram distributions of the psQDs. The histograms provide more information, for example confirming that both samples were quite heterogeneous, yet no clear distributions or conclusions can be discerned.
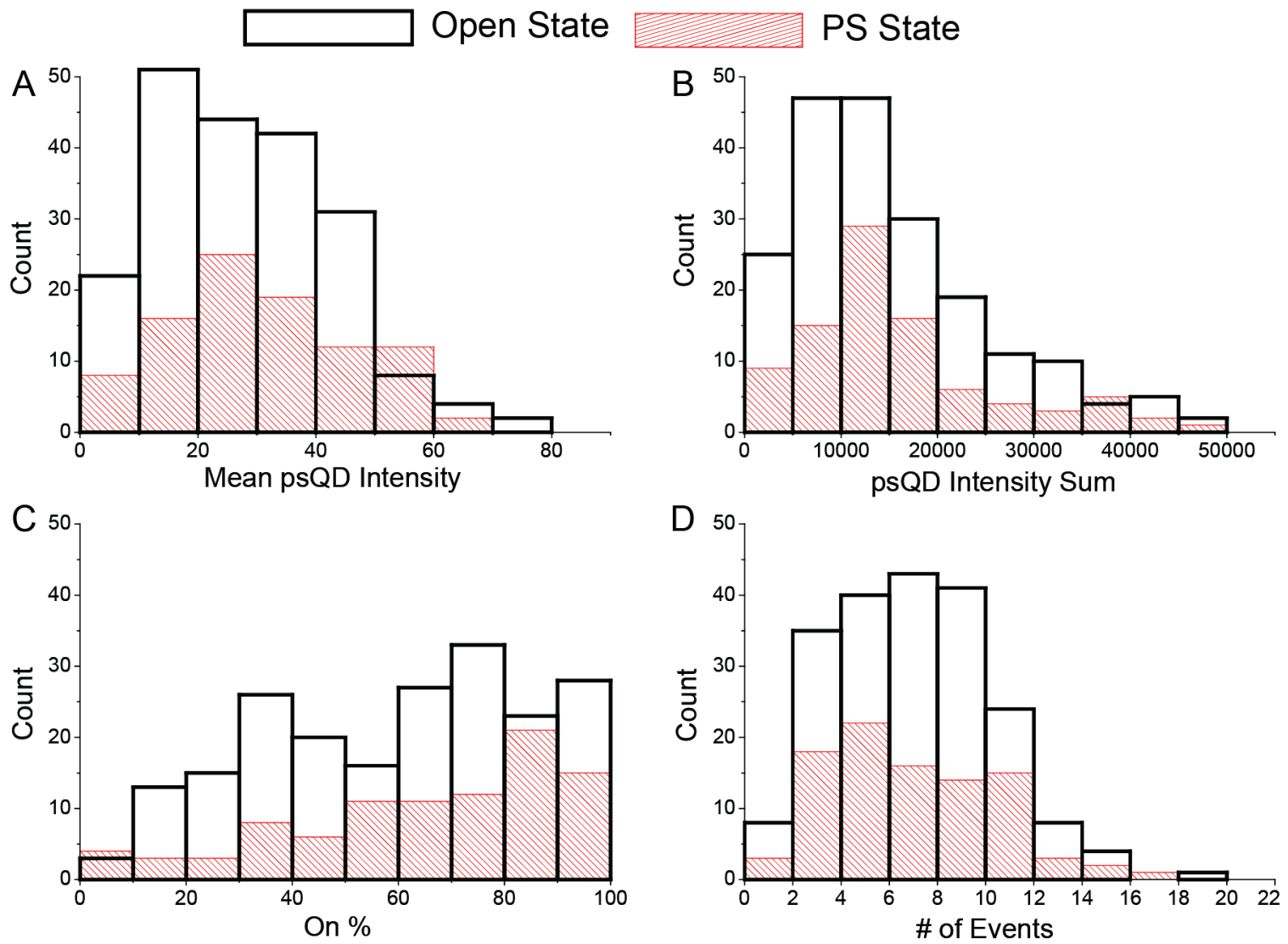

Figure 6: Histograms of PCaoc psQDs.

Due to the fact that the maximum and minimum intensity of psQDs does not vary much within a sample the mean and sum intensity principally reflects the amount of time the psQD is ON.

The dual-color psQDs (CdSe/CdS/ZnS core-shell-shell QD coated with PMA 7PCahx 75C12 $0.3 \mathrm{Al}$ ) were imaged next, at a higher concentration and with a total of 8 fields per state so as to improve the statistics.

Table 2: Dual-color psQDs. Presented uncertainties correspond to the sample standard deviation.

\begin{tabular}{lll}
\hline & Open State & PS State \\
\hline Sum of all Fields [counts x 10 ${ }^{5}$ ] & 80.0 & 60.4 \\
Mean Sum of Fields [counts x $10^{5}$ ] & $10.0 \pm 2.0$ & $7.5 \pm 3.0$ \\
\# of psQDs & 692 & 376 \\
Mean psQD Intensity [counts] & $19 \pm 13$ & $20 \pm 14$ \\
Mean ON \% & $47 \pm 29$ & $46 \pm 26$ \\
Mean \# of Events & $4.7 \pm 4.2$ & $8.0 \pm 4.5$ \\
\hline
\end{tabular}


Examination of individual psQDs again reveals no clear differences. The dual-color psQDs showed a $48 \%$ intensity difference in ensemble solution measurements. In comparison, the overall intensity detected by SPM was only modulated by $25 \%$ in the PS state. At the same time the number of psQDs observed in the PS state was 55\% of those detected in the open state. The disparity between the $48 \%$ ensemble and 25\% SPM determination was surprising since the first sample showed an increased difference in the SPM measurements. The histogram of the psQD intensity sum (Figure 7B) provides further insight. There is a small sub-set of extremely bright psQDs (inset of 7B) in the PS state, in fact 5 psQDs (1.3\% of population) make up $\sim 10 \%$ of the total intensity. It is possible that due to the higher concentration they are in fact multiple psQDs agglomerated together. Why the ultrabright psQDs were observed in the PS state as opposed to the open state is unclear.

The histogram distributions of the psQDs show a slight difference in the ON/OFF ratio and blinking statistics. The open state had a large number of psQDs (15\%) that were almost always ON. Surprisingly, examples of psQDs with $>90$ ON\% time could be found that had as many as 8 blinking events during the time-trace. It was determined that 1 in 3 of these psQDs with $>90 \%$ ON time where artifacts created by an OFF state slightly above the selected threshold skewing the values. It can also be observed in Figure 7D that the open state has more psQDs with few blinking events. An in-depth look at the data did not uncover any artifacts or particular reason as to why the distribution is shifted. In fact psQDs with few blinking events enclose the whole breadth of intensity and ON\% values.

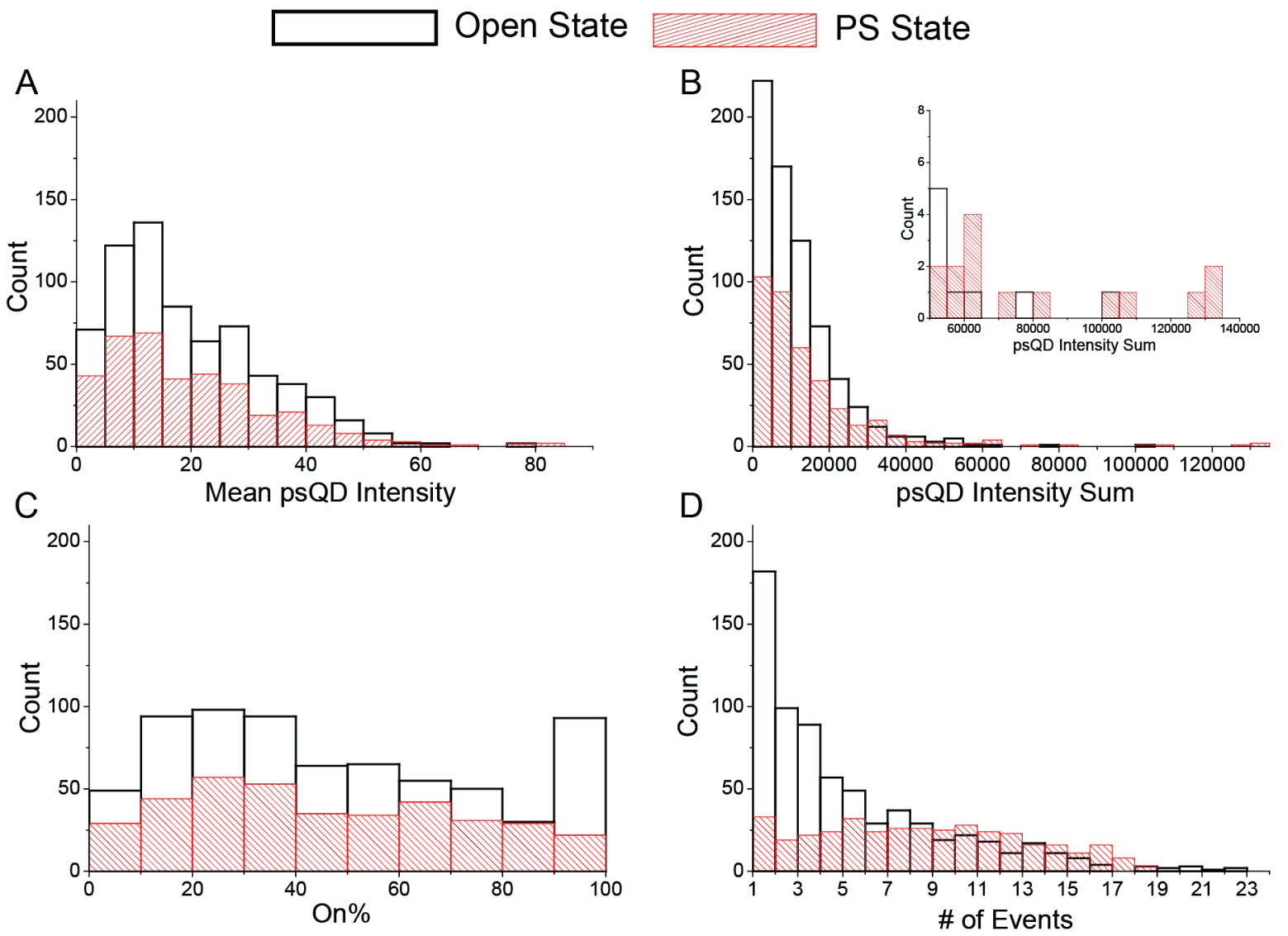

Figure 7: Histograms of dual-color psQDs. 
The end point characterization was successful, in that we were able to conclude that in the PS state a fraction of the psQDs were forced into a stable dark state. Photoswitching experiments were undertaken expecting to detect the change in the number of psQDs.

\subsubsection{Photoswitching Experiments}

For photoswitching experiments the same set-up and samples were utilized. A Nikon Intensilight $\mathrm{Hg}$ Precentered Fiber Illuminator supplied the photoswitching irradiation, the selected filters were: UV (340 $\pm 10 \mathrm{~nm})$ and Visible $(510-540 \mathrm{~nm})$. The experiments were realized by converting the open state to the PS state or vice versa. We attempted consecutive as well as alternating irradiations for either 10,30, or 60 seconds. A 5 second movie was taken initially and after each irradiation. The data analysis was similar to that discussed in Section 4.2.2.

Unfortunately, it was apparent even to the naked eye that the samples were merely undergoing photobleaching. In a representative experiment with 7 QDs in the initial field, after the first irradiation cycle there would only be 5 detected QDs and after a second cycle only 2-3 QDs remained. This was true both for controls and psQDs. It also appeared as if the visible or green light was the more efficient wavelength for photobleaching the QDs, probably due to the much higher intensity at $546 \mathrm{~nm}$ (strong $\mathrm{Hg}$ line) than at $340 \mathrm{~nm}$. As stated in Section 4.2.1 the QDs were dried on to the coverslip without any polymer coating. They were therefore particularly susceptible to photobleaching due to the exposure to oxygen, which would have greatly enhanced the rate of photobleaching. ${ }^{9}$ Future experiments will be carried out in aqueous medium with the psQD anchored to the glass through a biotin-streptavidin interaction. We anticipate a significant reduction in the rate of photobleaching.

\subsubsection{Secondary Observations}

The SPM provided further proof that the $400 \mathrm{~nm}$ excitation of the Alexa647 in the dual-color psQDs is indeed via a FRET mechanism. The Alexa647 on the dual-color psQD was excited directly using the $647 \mathrm{~nm}$ laser. Afterwards they were excited with the $485 \mathrm{~nm}$ laser, utilizing filters to isolate the Alexa647 emission, the blinking process of the $485 \mathrm{~nm}$ excitation followed a tendency much closer to that of the QD. It could also be observed that after 60 seconds of imaging with the 647 laser line most of the Alexa647 was bleached. The QDs did not photobleach during imaging, only during the irradiation, demonstrating their superior photostability.

One can consider whether the QD excitation was transforming the psQDs from the dark state to an emitting state. In Chapter 3 a pcFRET model was discussed in which efficient FRET and the large absorption cross-section of the QDs lead to a facile reversal of the PCs back to the open state during UV irradiation. In ensemble measurements in a spectrophotometer this effect was not observed, probably due to the very low light intensities involved, yet it is possible that at the single psQD level the phenomenon would be detected. An excitation laser would therefore not only read out the psQD but also switch the dark psQD to the ON state. 
A simple test for this hypothesis is to split the time trace into two parts $t_{1 / 2}$ and $t_{2 / 2}$ representing the first $50 \%$ of the time trace and the second $50 \%$ respectively. The psQDs were identified utilizing a maximum intensity projection of every frame of the movie, making its detection independent of when it begins to emit. If a psQD was being switched from a dark state during illumination, then there should be no emission until an arbitrary point of the movie $\left(t_{i}\right)$, where $t_{i}>t_{o}$. If we assume that the blinking statistics are not modified once the psQD is switched to the emitting state then the emission in $t_{2 / 2}$ should be higher since $t_{1 / 2}$ is in fact $t^{\prime}{ }_{1 / 2}=t_{1 / 2}-t_{i}$. Inspection of the PS state samples and comparison of the intensities do not indicate such an effect, it appeared as if the intensities were distributed randomly. In fact $54 \%$ of the psQDs had higher intensities in $t_{1 / 2}$ of the time trace. These results can be understood considering that the $488 \mathrm{~nm}$ line is not the most efficient wavelength for QD excitation and in view of the low photoreversal quantum efficiency.

\subsection{Conclusions}

The literature has many examples of QD SPM, ${ }^{10}$ yet that single psQDs can be readily imaged in a simple commercially available microscope is encouraging in terms of their potential utilization in more complex systems.

Initial studies support a model in which photocyclization shifts psQDs into long lived dark states that do not emit. At the same time, the inherent properties of the emitting psQDs, i.e. intensity, blinking statistics, and ON/OFF ratios, are not modified by the ensemble photostate. However, the conditions for conducting SPM experiments on psQDs must still be optimized, for example by establishing conditions under which single particle photoswitching can be observed without photobleaching. Using a solution based imaging medium, instead of the free exposure to air employed to date, should isolate the samples from oxygen and thus reduce photobleaching. The use of short light pulses and/or reduced intensity should also be beneficial.

The data analysis was based on other examples in the literature ${ }^{11}$ but optimized for the psQD system. Slight modification in the protocols will undoubtedly lead to different operational parameters and thus numerical values, but the conclusions are robust and were not modified by utilizing different cut-off or selection criteria.

Dark state fluorophores with extended OFF times are optimal for STORM as they allow for the interrogation of a single reporter dye. ${ }^{12}$ psQDs are bright and stable, photobleaching much slower than Alexa647, which is presently one of the gold standards for STORM imaging. ${ }^{12}$ They can also be excited with a variety of available lasers and their narrow emission makes them useful in multiplexed systems. The excitation laser does not switch the psQD state, and thermal reversion has not been observed, such that control of the psQD emission is "in hands of the operator". 


\section{References}

1. Moerner, W. E. A Dozen Years of Single-Molecule Spectroscopy in Physics, Chemistry, and Biophysics. J. Phys. Chem. B 2002, 106, 910-927.

2. Kapanidis, A. N.; Weiss, S. Fluorescent Probes and Bioconjugation Chemistries for Single-Molecule Fluorescence Analysis of Biomolecules. J. Chem. Phys. 2002, 117, 10953-10964.

3. Díaz, S. A.; Menendez, G. O.; Etchehon, M. H.; Giordano, L.; Jovin, T. M.; Jares-Erijman, E. A. Photoswitchable Water-Soluble Quantum Dots: pcFRET Based on Amphiphilic Photochromic Polymer Coating. ACS Nano 2011, 5, 2795-2805.

4. Zijlstra, N.; Blum, C.; Segers-Nolten, I. M. J.; Claessens, M. M. A. E.; Subramaniam, V. Molecular Composition of Sub-Stoichiometrically Labeled A-Synuclein Oligomers Determined by SingleMolecule Photobleaching. Angew. Chem. Int. Ed. 2012, 51, 8821-8824.

5. Diaspro, A., Confocal and Multiphoton Microscopy. Royal Society of Chemistry: 2004.

6. Stefani, F. D.; Hoogenboom, J. P.; Barkai, E. Beyond Quantum Jumps: Blinking Nanoscale Light Emitters. Phys. Today 2009, 62, 34-39.

7. Schneckenburger, H. Total Internal Reflection Fluorescence Microscopy: Technical Innovations and Novel Applications. Curr. Opin. Biotech. 2005, 16, 13-18.

8. Díaz, S. A.; Giordano, L.; Jovin, T. M.; Jares-Erijman, E. A. Modulation of a Photoswitchable DualColor Quantum Dot Containing a Photochromic FRET Acceptor and an Internal Standard. Nano Lett. 2012, 12, 3537-3544.

9. Durisic, N.; Wiseman, P. W.; Grütter, P.; Heyes, C. D. A Common Mechanism Underlies the Dark Fraction Formation and Fluorescence Blinking of Quantum Dots. ACSnano 2009, 3, 1167-1175.

10. Empedocles, S. A.; Norris, D. J.; Bawendi, M. G. Photoluminescence Spectroscopy of Single CdSe Nanocrystallite Quantum Dots. Phys. Rev. Lett. 1996, 77, 3873-3876.

11. Durisic, N.; Godin, A. G.; DerrelWalters; Grütter, P.; Wiseman, P. W.; Heyes, C. D. Probing the "Dark" Fraction of Core-Shell Quantum Dots by Ensemble and Single Particle pH-Dependent Spectroscopy. ACSnano 2011, 5, 9062-9073.

12. Dempsey, G. T.; Vaughan, J. C.; Chen, K. H.; Bates, M.; Zhuang, X. W. Evaluation of Fluorophores for Optimal Performance in Localization-Based Super-Resolution Imaging. Nat. Methods 2011, 8, $1027-$ 1036. 


\section{CHAPTER 5: BIOLOGICAL TARGETING OF PHOTOSWITCHABLE QUANTUM DOTS}

\section{Introduction}

Fluorescence microscopy is an essential tool in the investigation of structural and dynamic information at the cellular level. As the questions posed by scientists become more complex the search for better fluorophores becomes critical. My work has focused on developing and investigating the physical and chemical properties of psQDs whereas the primary interest of the lab has been their application as cellular probes. A series of initial experiments were realized with the aim of testing the potential of psQD in fluorescence imaging. Cell culture and preparation was realized by Lic. Martin Toscani (Quimica Biologica, FCEN-UBA, Buenos Aires, Argentina) or Dr. Donna Arndt-Jovin (Laboratory of Cellular Dynamics, MPIbpc, Göttingen, Germany); subsequent procedures were carried out in conjunction with my collaborators or by me.

The information that can be gleaned from a fluorescence image is limited in part by the specificity of labeling. It is crucial that the fluorophore report on the parameter(s) of interest and minimize background emission. Indiscriminate uptake was eschewed in favor of developing more elegant specifically targeted psQDs. Two distinct targeting methodologies were attempted and are presented in Section 5.1 and 5.3. The targeted psQD preparation and purification are presented, and then the imaging experiments and results are presented for each method in turn.

The selected target was the epidermal growth factor receptor (EGFR, ErbB1, HER1), a member of the ErbB family of receptor tyrosine kinases. ${ }^{1}$ EGFR is studied for its far-reaching biomedical importance due to the overexpression and/or mutation found in various types of cancer. For example, over one third of all carcinomas, which is the most frequent type of cancer, present very high levels of EGFR. ${ }^{2}$ For the discovery of the Epidermal Growth Factor (EGF), the activating ligand of EGFR, Stanley Cohen was awarded the Nobel Prize in Physiology in 1986. The extracellular binding of EGF causes the EGFR to dimerize, either as a homodimer or a heterodimer with other ErbB members. ${ }^{3}$ The active dimer autotransphosphorylates specific tyrosine residues in the cytoplasmic C-termini, thereby creating binding sites for adapter proteins that stimulate various signaling cascades, for example the MAPK, Akt, and JKT pathways regulating cell motility, differentiation, and division. ${ }^{4}$ The EGFR system is well established within the lab and multiple reports utilizing QDs as labels for its study and tracking have been published. ${ }^{5,6}$

The psQDs were imaged on both live and fixed cells and photoswitching experiments were realized using different light sources. 


\section{1 psQDs Targeted Through Post-Coating Functionalization}

When suspended in alkaline buffer solutions the psQDs present a negative charge due to the polymer carboxyl groups. This negative charge creates a repulsive force that keeps them from aggregating, while at the same time the large number of carboxyl groups provides a means for conjugation to other molecules. ${ }^{7}$ The modification of a few groups will not neutralize the psQD although excessive modification promotes aggregation. The modification of the psQD surface after polymer coating and re-suspension is known as a post-coating functionalization. This strategy could be applied by a downstream user to tailor any psQD to his specific purpose. Within sections 5.1 and 5.2 the characterization and results of the post-coating functionalization based on the first generation psQDs (Section 3.1) are presented.

As mentioned above, the exposed polymer surface of psQDs are rich in carboxyl groups, around 1000 per psQD, and these can be targeted for further functionalization. Carboxyl groups are not very reactive, but carbodiimide crosslinker chemistry provides an activation pathway for the formation of amide bonds with primary amines. For use in aqueous conditions the most common reagent is EDC (1-ethyl-3-(3-dimethylaminopropyl) carbodiimide) in combination with Sulfo-NHS (N-Hydroxysulfosuccinimide).

EDC forms an o-acylisourea, the reactive species which is either hydrolyzed back to the carboxyl group or reacts with an amine to form the amide bond (Scheme 1). The EDC is consumed during the reaction, but does not add any atoms between the two reagents and is therefore referred to as a zero-length linker. Sulfo-NHS increases the efficiency of coupling by limiting the competing hydrolysis pathway. The Sulfo-NHS reacts with the o-acylisourea to create a Sulfo-NHS-Ester, which can be displaced by a primary amine. ${ }^{8}$

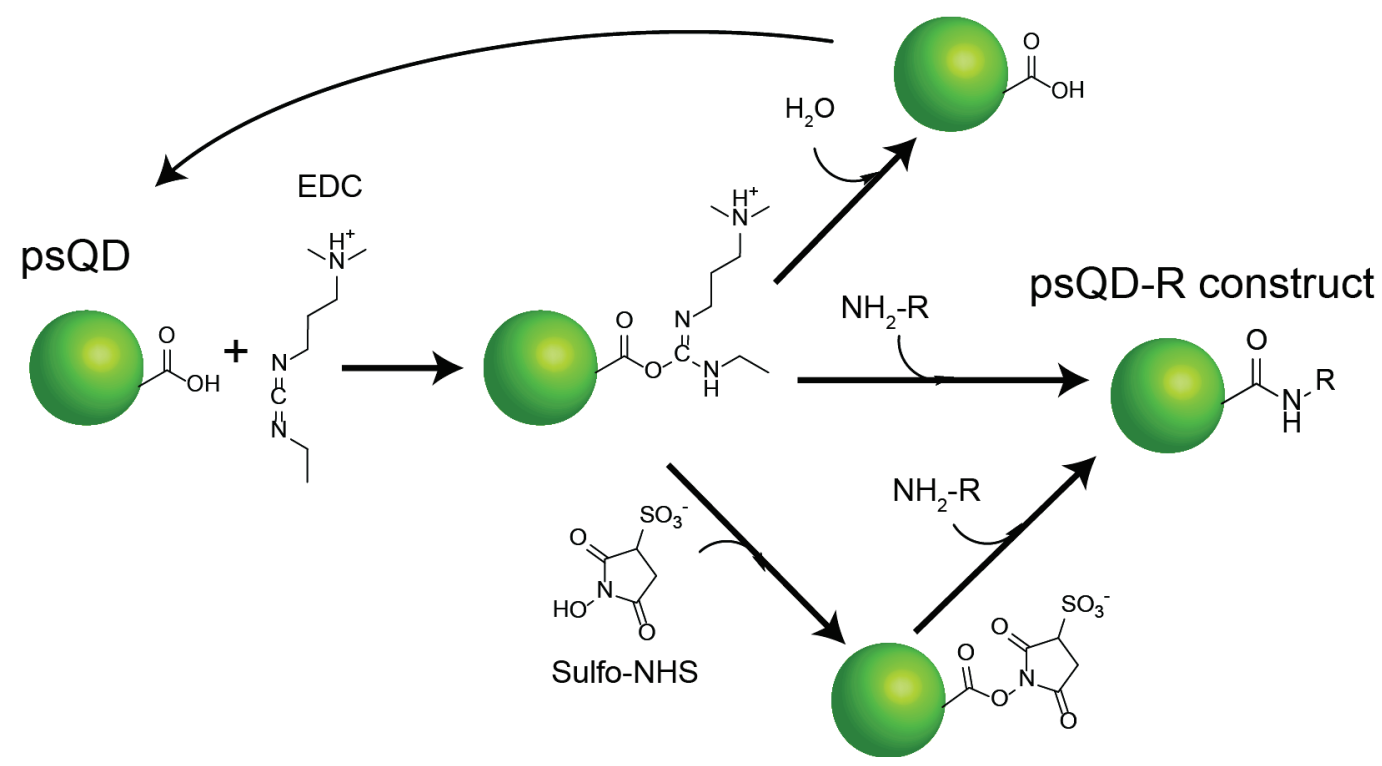

Scheme 1: EDC conjugation of psQD with primary amine. 
EDC is most efficient in acidic conditions ( $\mathrm{pH}$ 5) but the psQDs are limited to an alkaline buffer for stability. Thus, the $\mathrm{pH}$ was lowered to the 7-8 range and excess EDC and Sulfo-NHS (approx. 2000-fold) were utilized to compensate. ${ }^{9}$ Multiple preparations of four separate psQD functionalizations were attempted. An outline of the preparation and most important properties of each functionalization are presented in the following sub-sections. Each specific preparation will be referred to as a psQD construct, e.g. a psQD directly conjugated to streptavidin will be known as a psQD-streptavidin construct. Section 5.2 presents the imaging results from these preparations.

\subsubsection{Conjugation of Texas Red Cadaverine to psQDs}

EDC reaction conditions (mentioned above) were optimized with the use of psQDs and Texas Red (TR) Cadaverine dye. The TR dye has an absorbance maximum at $596 \mathrm{~nm}\left(\varepsilon=85000 \mathrm{M}^{-1} \mathrm{~cm}^{-1}\right)$ and emission maximum of $605 \mathrm{~nm}$. It was therefore easy to follow the conjugation by absorption and fluorescence spectroscopy. It is important to note the difference between the equivalents (eq.) that were added to the conjugation reaction and the amount of functional moieties that were covalently bound to the psQD. When eq. are discussed it is in reference to the proportions in the conjugation reaction. If excess dye was added to the conjugation reaction ( $>30$ TR eq. per psQD), aggregation was observed that was not reversed upon alkalization of the medium. With TR/psQD $<15$ eq. there was no aggregation observed.

Conjugated psQDs were purified in the same manner as other psQDs (Section 3.1.1). The psQD fraction was recovered in the 45 min fraction in the size exclusion purification step; small molecules such as TR and EDC eluted after 90 min. A second option was the utilization of Pierce Slide-A-Lyzer mini dialysis units $(10,000 \mathrm{MWCO})$ for the elimination of unconjugated reagents. Dialysis was best suited for smaller molecules such as TR, EGF (5.1.2) and biotin (5.1.4). The conjugation was realized on previously purified psQDs, ensuring that excess polymersomes did not interfere with the reaction.

Irradiation of $\mathrm{psQD}$ constructs showed that the original $\mathrm{psQD}$ emission modulation of $\sim 35 \%$ was unaffected by any of the tested surface conjugations. In a similar manner the photokinetics of PC conversion were not modified. Characterization therefore focused on the surface/functionalization properties of the psQD constructs.

The TR conjugation was purified by the above mentioned chromatography method and was then confirmed by spectroscopic measurements. The absorbance spectra allowed for the determination of the psQD to TR ratio. The final ratio was in the range of one TR per psQD, and the psQD-TR construct was stable in the same general conditions as unmodified psQDs. The fluorescence of the QD and the TR tracked each other throughout the fractions collected in the purification process, (See Figure 1). As it was previously observed that unbound TR eluted at $90 \mathrm{~min}$, this was strong evidence for conjugation. 

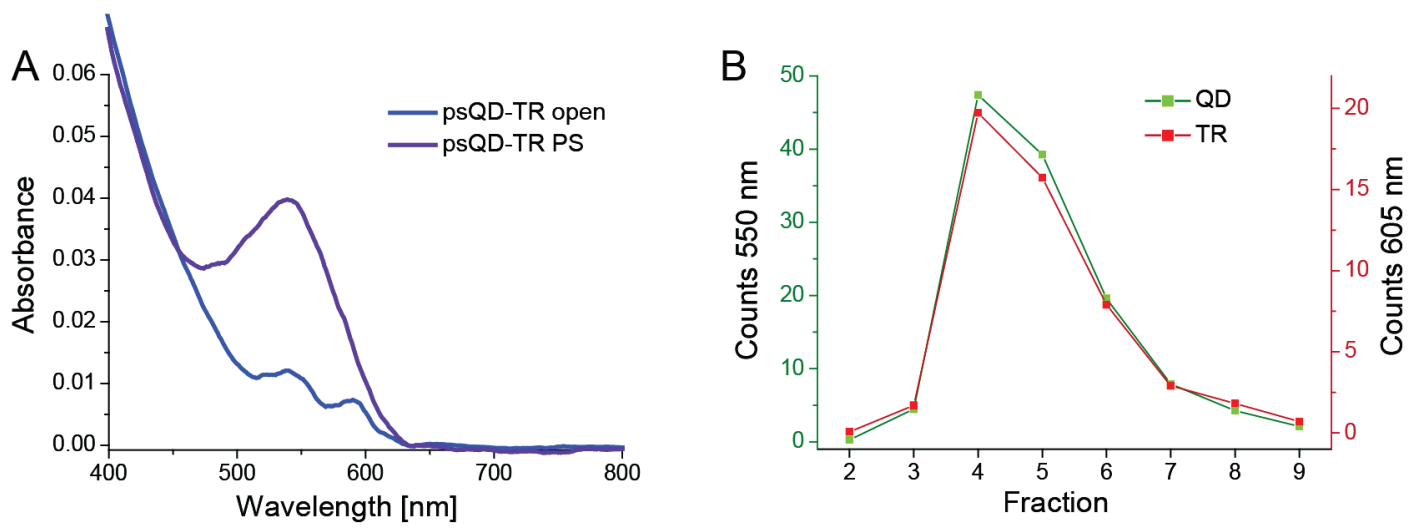

Figure 1: psQD-TR construct with 0.67TR per psQD. A) Absorbance spectra of the construct in the open and PS states. B) Emission counts of fractions purified through size-exclusion column. QD excitation, $400 \mathrm{~nm}$. TR excitation, $580 \mathrm{~nm}$.

The psQD-TR construct served to determine the general conditions of conjugation, but was of no specific interest as a functional construct. The TR did not provide the same benefits as a secondary fluorophore as the Alexa647 did in the dual-color psQDs (Chapter 3). The TR fluorescence was quenched by the PC photoconversion and the change in the QD-TR peak ratio was only $35 \%$ as compared to the $100 \%$ seen for QD-Alexa647. The psQD-TR constructs lacked any targeting capabilities, required for the intended biological applications. The constructs presented in the following sub-sections utilized similar conjugation conditions but involved biologically relevant moieties.

\subsubsection{Conjugation of Epidermal Growth Factor to psQDs}

When observing in vivo biological targets smaller fluorescent probes are preferred as they will have fewer secondary interactions with the surrounding environment. To keep the construct as small as possible a direct conjugation of the target ligand was attempted. EGF is a 53 amino acid peptide that contains two lysines (K28, K48), amino acids with primary amine side chains, and the amino terminal $\alpha$-amine for conjugation to the carboxyl groups. A recombinant humanEGF (Peprotech) was added to the conjugation solution at a stoichiometry of 150 eq. per psQD; the actual conjugation ratio was not determined. None of the EGF conjugation attempts resulted in aggregation; this suggested that the conjugation must have been quite inefficient and that the amount of EGF covalently bound to the psQDs was in fact quite low. DLS determinations reported a slight increase in the hydrodynamic diameter to $19 \pm 6 \mathrm{~nm}$ as compared to the $15 \pm 3 \mathrm{~nm}$ for the unmodified psQD, suggesting that some conjugation did take place. However, direct conjugation of the EGF resulted in a complete loss of activity as an EGFR ligand, as determined by the complete lack of psQD binding to cells expressing EGFR. It was very difficult to limit the conjugation to the amino terminal alpha amino side chain and derivation of the two internal lysines is known to be detrimental to the biological function of the EGF. An alternative is the use of mouse EGF which can bind to human EGFR but only presents the amino terminal $\alpha$-amine for conjugation. ${ }^{10}$ It may also be that the psQD creates a sterical limitation making it impossible for the EGFR receptor pocket to envelop the EGF; in this case extended linkers may 
provide a solution. No test, beyond cellular uptake, was attempted to determine the presence of EGF on psQDs. As direct conjugation proved ineffective, indirect conjugation was attempted, as described in the next two sub-sections.

\subsubsection{Conjugation of Streptavidin to psQDs}

The work of Lidke et $a l^{5}$ demonstrated that EGFR could be targeted by QDs using a streptavidin and biotin-EGF system. Since the psQD-EGF construct was inactive as a ligand, the next step was to attempt to recreate the QD-Streptavidin plus biotin-EGF system using the psQDs. Streptavidin is a $60 \mathrm{kDa}$ homo-tetramer, guaranteeing the availability of an active binding pocket independently of the conjugation position.

Initially the successful preparation of the psQD- streptavidin construct required a modification of the employed protocol. The conjugation required high concentrations of all reagents, yet these same conditions favored cross-linking driven aggregation. The psQD (1 eq., $140 \mathrm{nM}$ ), NHS (80000 eq., 10 $\mu \mathrm{M}$ ), and streptavidin (18 eq., $2.5 \mu \mathrm{M}$ ) were mixed in an eppendorf before the addition of the EDC $(80000$ eq., $10 \mu \mathrm{M})$. The reactions proceeded for $10 \mathrm{~min}$ at $\mathrm{RT}$ and were then quenched with 2 mercaptoethanol. In the absence of the quenching reagent, a reaction allowed to continue past 10 min led to cross-linking and massive aggregation of the psQDs, such that by $15 \mathrm{~min}$ of reaction time there would be zero colloidal psQDs. The high concentration conditions were required as lower concentrations did not produce efficient conjugation with the streptavidin. After subsequent experimentation, slightly modified conjugation conditions were found. The psQDs were prepared in a $7.9 \mathrm{pH}$ buffer as compared to the previous $\mathrm{pH} 9.0$ and only 3300 eq. of Sulfo-NHS and EDC were utilized, the reaction was slowed down 3-fold.

The size-exclusion purification step also allowed a quantification of the conjugated streptavidin. Unbound streptavidin eluted through the column in 75 min. A calibration injection of streptavidin diluted in buffer allowed the areas of the 75 min peaks in the reaction and calibration injection to be compared to determine conjugation efficiency. This was possible with streptavidin, as opposed to EGF or TR, as the peak did not overlap with the excess EDC or NHS-ester. If it was assumed that the peak decrease was due essentially to psQD conjugation, than successful psQDstreptavidin construct preparations had slightly less than 2 streptavidin per psQD.

The psQDs containing streptavidin were characterized by using a fluorescence quencher that overlapped the emission of the QD fluorescence. Biotin-Atto540Q (Atto-Tec GmbH), capable of quenching the QD emission through FRET, was added to streptavidin conjugated psQDs and control samples. A polymer coated QD was used as the baseline and when this baseline was applied to the data only the "successful" psQD-streptavidin constructs demonstrated decreased emission. An example is presented in Figure 2B where 12 picomoles of psQD-streptavidin were titrated by the Biotin-Atto540Q. At 20 picomoles of quencher the FRET appears to have been saturated, signifying 
very low conjugation efficiency where only 3 out of 5 psQDs had a streptavidin conjugated to it. To confirm that the quenching was due to the streptavidin-biotin bind bringing the Atto540Q near enough to interact with the QD, the data was fit to the FRET equation (Section 3.2, Equation 1). Until the point of saturation, $>20$ picomoles, the data fit very well. DLS determinations yielded a $34 \pm 7$ hydrodynamic diameter, consistent with the larger size of streptavidin compared to EGF.

A

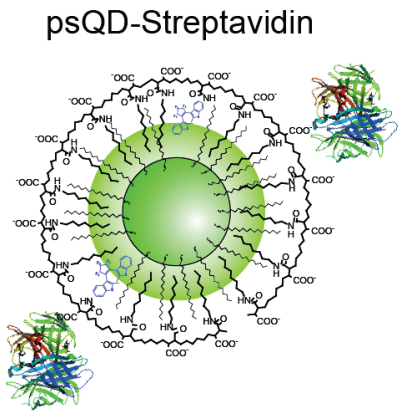

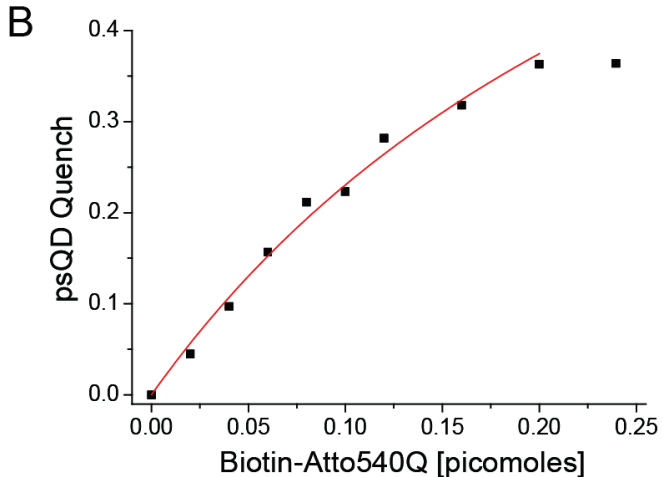

Figure 2: A) Scheme of psQD-streptavidin construct (to scale). Streptavidin image adapted from ${ }^{11}$ B) Emission quenching of 12 pmoles of psQD-Streptavidin construct upon addition of Biotin-Atto540Q.

A commercial biotin-EGF (Molecular Probes) containing a single amino terminal biotin adduct was used to target the psQD-streptavidin constructs (see Section 5.2). ${ }^{6}$ The psQD-streptavidin construct was the most successful of the preparations when it came to imaging, but its preparation was also the most cumbersome. Streptavidin was too large to eliminate with dialysis, obliging the construct to be purified by size-exclusion column, which had a much smaller final yield, and crosslinking leading to aggregation also caused the loss of whole batches of preparations. As in the case of EGF an alternative path to indirectly conjugate the streptavidin to the psQD was attempted (see following subsection).

\subsubsection{Conjugation of Biotin to psQDs}

The cross-linking in the psQD-streptavidin construct was driven by the large size and multiple conjugation sites of the streptavidin; an alternative strategy in which biotin was conjugated to the psQD was investigated. A commercial linker with a primary amine on one end and biotin on the other (Thermo \#21340) was utilized to conjugate a biotin to the psQDs. The TR experiments had demonstrated that excessive conjugation was not desirable; thus, the conjugation was limited to $<10$ biotin eq. per $\mathrm{psQD}$. As the amine-biotin only had one conjugation position psQD crosslinking was avoided. A streptavidin molecule could then indirectly be bound to the psQD through the biotin. The psQD-biotin constructs were targeted through the addition of streptavidin that had the other binding pockets occupied by a biotin-EGF. The tested ratio was 1: 0.5: 0.8 of psQD-biotin to streptavidin to Biotin-EGF respectively. The mixture was prepared before addition to the cells.

There were multiple ways to test if the psQDs presented exposed biotin (two examples are given in Section 5.3). Regrettably, at the time of their preparation the constructs of the post-coating 
functionalization of psQDs with biotin were not adequately characterized due to time constraints. Section 5.3 focuses on an improved, simpler and more versatile method for preparing psQDs with exposed biotin on the surface, making post-coating functionalization with biotin an unattractive strategy.

\subsection{Fluorescence Imaging Experiments with Post-Coating Functionalized psQDs}

Two cell lines were selected for imaging; the first was A431, an epidermoid carcinoma model cell line. A431 expresses $2.3 \cdot 10^{6}$ EGFR on their plasma membranes, making it an ideal system for testing the targeted psQDs. ${ }^{12}$ The second cell line was a stably transfected CHO (Chinese hamster ovary) line expressing a chimeric EGFR with an amino terminal ACP (acyl carrier protein) tag. The phosphopantetheine transferases conjugates CoA derivatives to the hydroxyl group of serine 36 on the ACP tag. ${ }^{13}$ The enzyme accepts adducts attached to the terminal cysteine thiol of Coenzyme A and transfers the substituted phosphopantetheine moiety (Scheme 2). ${ }^{14}$
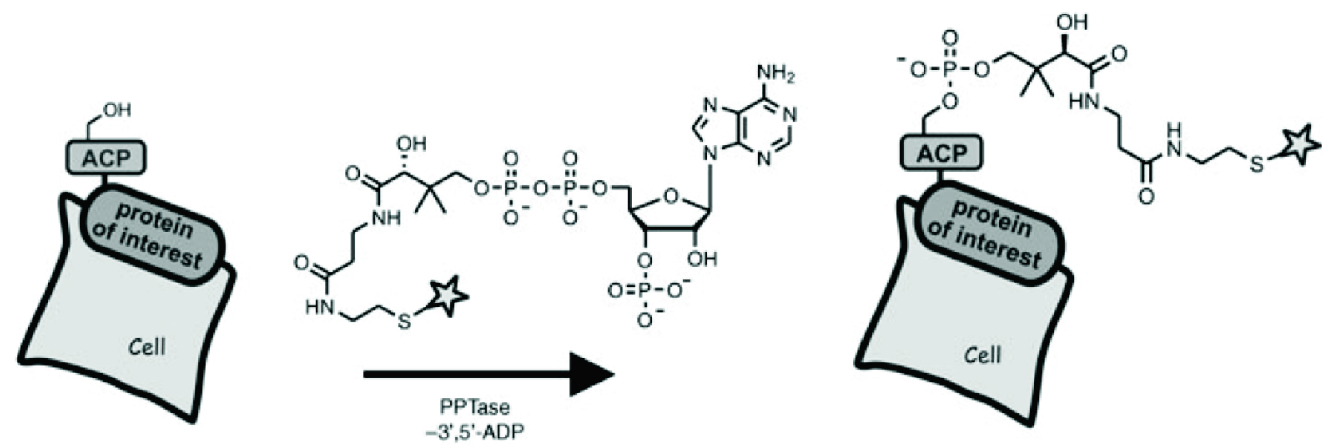

Scheme 2: Labeling of ACP fusion proteins on cell surfaces. The star represents different possible labels. ${ }^{14}$ (C)2005 WILEY-VCH

We used a CoA-biotin, which covalently attached a biotin to the EGFR exclusively on the cell surface, the internal protein not being accessible to either the enzyme or the substrate. The psQDs with streptavidin were then targeted to the modified EGFR on the ACP-tagged CHO cells in the absence of EGF. All constructs were utilized in the nanomolar range [1-30 nM] to perform the imaging experiments.

Imaging was realized on a commercial confocal scanning microscope or utilizing a programmable array microscope ${ }^{15}$ built in the lab. Photoswitching experiments were attempted with the microscope light sources, either with whole field Hg lamp irradiation or partial field laser irradiation.

The A431 cells were starved for $30 \mathrm{~min}$ before the $30 \mathrm{~min}$ incubation of the psQD-construct at RT. Multiple washes were carried out before imaging. The CHO cells were biotinylated as described 
above. After 15 min the cells were washed (3x) and then incubated with the psQD-construct for 30 min followed by two washes before imaging.

When working with targeted fluorophores the first control is the determination of unspecific binding or uptake. For this control unmodified psQDs, which only present the negative charges from the carboxyl group, were chosen. Repeated experiments demonstrated that the cell lines lacked significant psQD uptake or binding during 30 min incubations at RT. The cell surface, being negatively charged, likely repels the negatively charged surface of the psQD.

The second test was a positive control of the cell preparations. Commercially available Qdot 565ITK Streptavidin conjugates (Invitrogen) were used, either targeted using the Biotin-EGF for A431 cells or directly to the EGFR-Biotin on the ACP-EGFR-CHO cells. Figure 3A shows an example of the positive control. Photoswitching experiments with these commercial QDs did not show cyclical photomodulation.

Multiple imaging experiments were realized with the different constructs and with varying success. The psQD-TR construct, similar to the negative control, did not bind to the cells. Neither did the psQD-EGF constructs. The psQD-streptavidin construct was the most successful and the experiments presented below were all with this construct.

Unfortunately, experiments with the cells gave mixed results which were not always possible to clearly interpret. Some of the results are presented below as well as a discussion of the problems encountered such as inconsistent uptake, psQD aggregation and QD bleaching.

The first experiment (Figure 3) was of live A431 cells targeted by a psQD-streptavidin construct in the PS state, i.e. the QD was quenched. The psQD-streptavidin construct was mixed with biotin-EGF in a 1 to 2 ratio and then added to the cell at RT. Care was taken to minimize ambient illumination; all preparations were covered with aluminum foil to avoid light exposure that could modify the state of the psQD construct. The construct was introduced in the quenched state and psQD brightening induced by exposure to visible light irradiation using the intense $546 \mathrm{~nm} \mathrm{Hg}$ line was observed. The cells were imaged with a Leica TCS SP5 confocal microscope using $405 \mathrm{~nm}$ diode laser source before and after $30 \mathrm{sec}$ of $515-560 \mathrm{~nm}$ (filter bandwidth) irradiation (Figure 3B,C, respectively). Analogous to what was observed in previous reports, in the presence of EGF the cells were activated and the EGFR complex along with the psQD constructs internalized. ${ }^{6}$ The two large endosomes, which contained multiple psQDs, were much brighter and easier to quantify. The constructs were $33 \%$ brighter after irradiation with green visible light as seen in Figure 3D. 

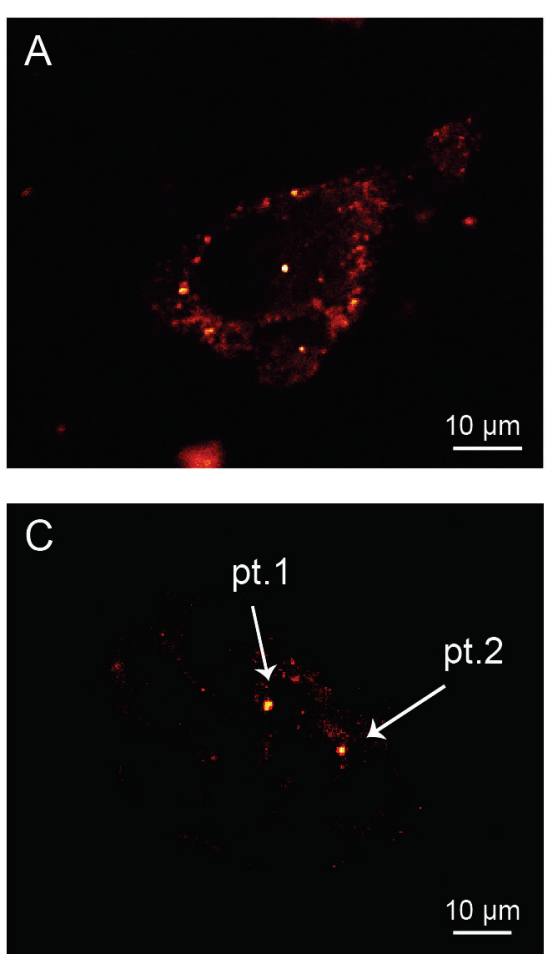

$\mathrm{D}$

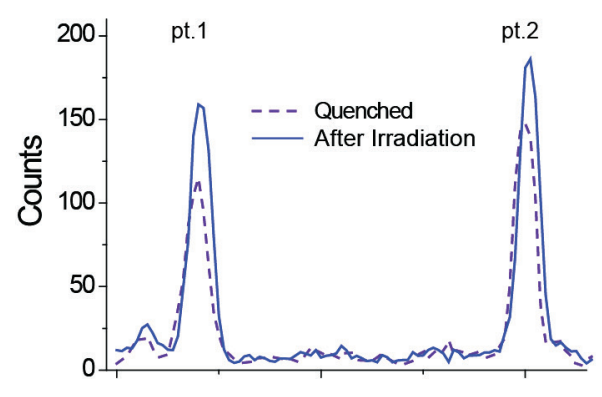

Figure 3: A) A431 cells targetted with commercial Qdot 565ITK Streptavidin conjugates (Invitrogen). B) A431 cells targetted with psQD-streptavidin constructs in the PS state. C) Same image as B after $30 \mathrm{~s}$ of 515-560 nm irradiation. D) Intensity profile of the selected endesomes in B and C.

Similar experiments were attempted in which the $561 \mathrm{~nm}$ diode laser line was utilized as the light source for de-quenching the psQDs in the PS state in a specific region of the cell. The quantification showed no definite difference between the irradiated and non-irradiated section of the cells. Possibilities include the photobleaching of the irradiated area, inefficient conversion with the diode laser line, or that the samples were not adequately quenched before the measurements.

A complete photocycle was realized on live ACP-EGFR-CHO cells biotinylated on the ACP tag and targeted by the psQD-streptavidin construct. In this case the cells were incubated with psQDstreptavidin in the open state, and irradiation was carried out with the $365 \mathrm{~nm} \mathrm{UV} \mathrm{Hg} \mathrm{line} \mathrm{for} 30 \mathrm{~s}$ and then with the $546 \mathrm{~nm} \mathrm{Hg}$ line for $30 \mathrm{~s}$. Table 1 and Figure 4 presents the results of the experiment. The UV irradiation drastically decreases all intensity parameters, while the visible irradiation increases the values, but not back to their original state.

Table 1: Fluorescence of psQD-streptavidin marked EGFR after UV and visible irradiations.

\begin{tabular}{lccc}
\hline & Initial & UV & Visible \\
\hline Total Counts & 72500 & 37000 & 45600 \\
Pixels above Threshold & 902 & 529 & 618 \\
Mean Pixel Intensity & 81 & 70 & 74 \\
Median Pixel Intensity & 73 & 67 & 69 \\
\hline
\end{tabular}




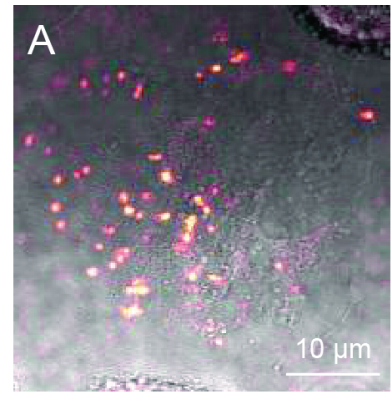

$\mathrm{E}$

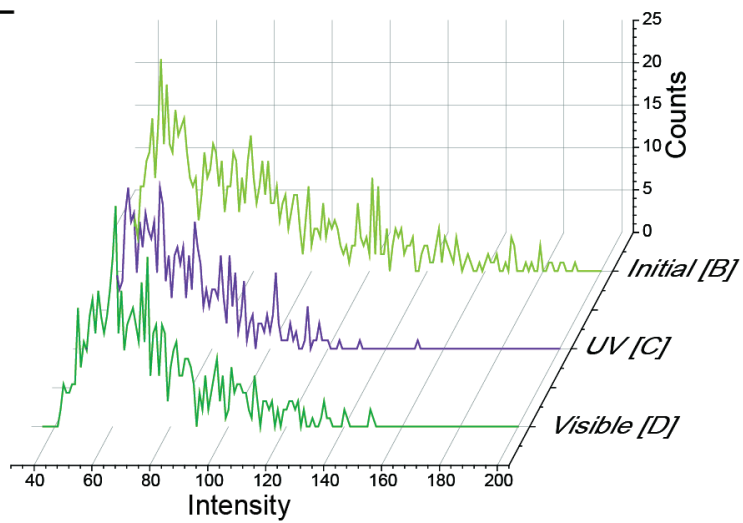

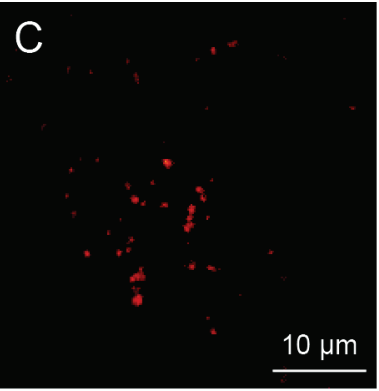

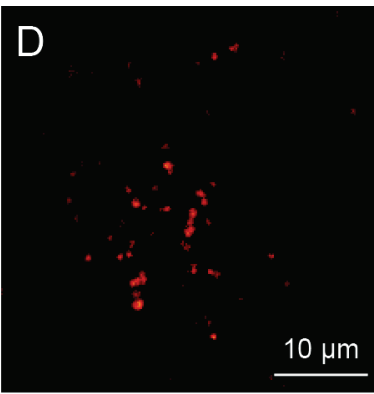

$\mathrm{F}$

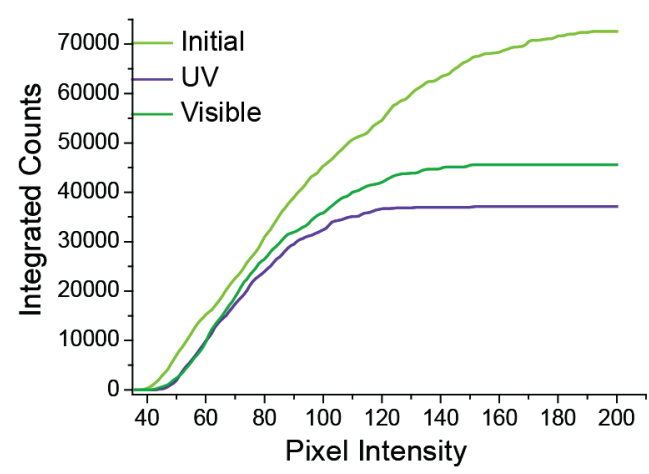

Figure 4: $\mathrm{CHO}$ cells marked with psQD-streptavidin constructs. All images have been background corrected and a threshold was applied. A) DIC and fluorescence overlay B) Initial (open state) C) After 30 seconds of UV irradiation. D) After $30 \mathrm{~s}$ of $515-560 \mathrm{~nm}$ irradiation. E) Histograms of pixel intensity. F) Integration of histograms presented in $\mathrm{E}$.

In Section 3.3.4 dual-color psQD samples in solution were irradiated with $365 \mathrm{~nm}$ light (the UV source available on the microscope) and it was observed that the maximum quenching was only $25 \%$ as opposed to the $45 \%$ obtained with $340 \mathrm{~nm}$ light. ${ }^{16}$ Therefore the $50 \%$ decrease in overall intensity observed in the microscope after UV irradiation most likely contained a photobleaching component, not only of the psQDs but of the background autofluorescence of the cell as well. After the $30 \mathrm{~s}$ of visible irradiation there was an increase in all the fluorescence signals. The number of pixels above the threshold increased as well as the mean and medium pixel intensities. The total intensity measured in the region of interest increased by $23 \%$ above the quenched value after visible irradiation. Though the irradiation and imaging conditions caused some photobleaching of either the psQD construct or the cell autofluorescence, making the interpretation of the results more complicated, the cyclical modulation of the probe intensity was observed.

In conclusion the post-functionalized psQDs demonstrated the ability to mark specific targets on live cells and initial results of photomodulation were obtained. Three different constructs were attempted with the intent of labeling the EGFR. The direct conjugation of the EGF ligand to the psQD created the smallest construct but also caused the inactivation of the EGF; thus, the construct was unable to bind to cells. The psQD-streptavidin construct was the most successful as a fluorophore. The drawback was the low yield in the preparation, due in large part to crosslinking as well as to the increased size of the probe (34 $\mathrm{nm}$ hydrodynamic diameter). The construct preparation and imaging 
conditions, especially for photoswitching, require further investigation before the psQD-constructs will be practical for more complex or unknown systems.

\section{3 psQDs Targeted Through Functionalized Polymer Coating}

As indicated above, post-coating functionalization of the psQD provided some initial success, but the process often led to aggregation and psQD crosslinking. The psQD must be purified before functionalization and as well as after post-functionalization, greatly reducing the final yield and making the process time consuming. By using a previously functionalized polymer for the QD coating these inconveniences can be avoided. For this purpose, a biotin functionalized photochromic comblike amphiphilic polymer, PMA 6PCala 75C12 1.3PEG-Bt, was prepared as detailed in Section 1.5.2. The QDs were then coated with this polymer using the same methodology used with the other polymers; the purification and characterization followed the same steps as was specified in Chapter 3 . The only observed difference was that the PEG made the single strand polymer more dispersible in the SBB buffer, such that the fraction of single strand polymer separated by the size-exclusion column was proportionally larger. The final construct was a psQD presenting a mean of 5-6 biotin molecules on the end of a PEG linker, denoted psQDbiotin. The surface biotin bound streptavidin molecules to the psQD. Streptavidin is a homo-tetramer, and thus was expected to present three other binding sites for additional biotin. The second biotin molecule can be bound to a ligand, such as biotin-EGF or the EGFR-ACP-biotin model featured in Section 5.2.

\subsubsection{Photophysical and Surface Characterization}

The photophysical properties of the psQDbiotin were determined in the same manner as common psQDs. The results are summarized in Figure 5 and Table 2.
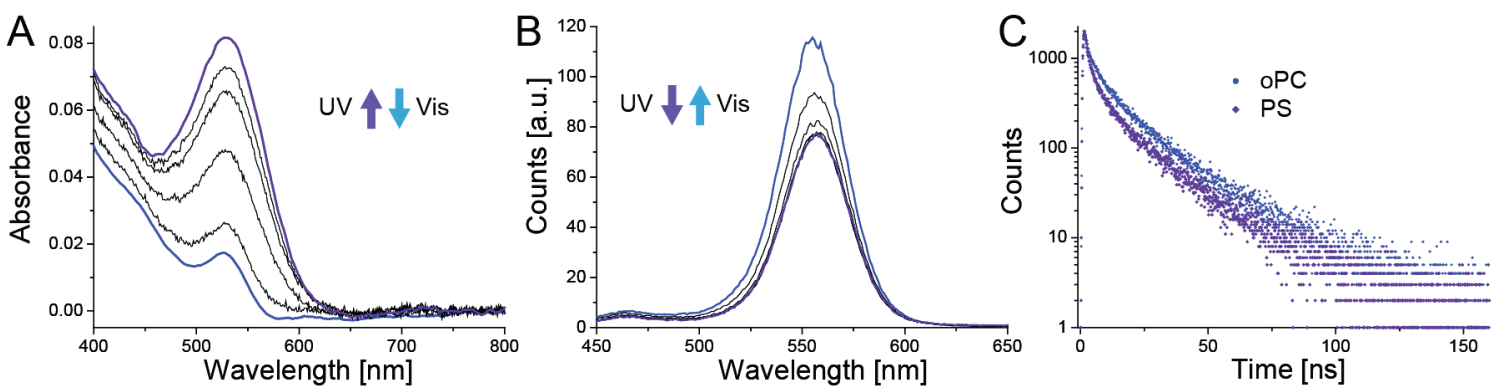

Figure 5: Spectroscopic monitoring of psQDbiotin. Irradiation was UV: 340 (irradiance $1.1 \mathrm{~mW} \mathrm{~cm}^{-2}$ ) and Vis: 545 (irradiance $6.2 \mathrm{~mW} \mathrm{~cm}{ }^{-2}$ ). Temperature, $20{ }^{\circ} \mathrm{C}$. (A) Absorbance spectra (B) Fluorescence spectra (C) Lifetime fluorescence spectra. 
Table 2: psQDbiotin Characterization

\begin{tabular}{lcccccc}
\hline & $\begin{array}{c}\text { Hydrodynamic } \\
\text { Diameter [nm] }\end{array}$ & $\begin{array}{c}\text { Quantum } \\
\text { Yield }\end{array}$ & $\begin{array}{c}\text { cPC/QD in } \\
\text { PS state }\end{array}$ & $\begin{array}{c}\text { Fluorescence } \\
\text { Modulation }\end{array}$ & $\begin{array}{c}\langle\tau\rangle_{\text {amp }} \\
{[\mathrm{ns}]}\end{array}$ & $\begin{array}{c}\langle\tau\rangle_{\text {int }} \\
\text { Modulation }\end{array}$ \\
\hline psQDbiotin & $17.5 \pm 1.5$ & 0.11 & 15 & $35 \%$ & $3.83 \pm 0.05$ & $30 \%$ \\
\hline
\end{tabular}

The biotin concentration on the polymer was quantified by NMR spectroscopy, but it was still necessary to determine if the psQDbiotin presented accessible biotins. Two experiments provided confirmation that the surface biotin could indeed bind to streptavidin.

The psQDbiotin and a control psQD where mixed with Streptavidin-Atto565 (Sigma-Aldrich) in SBB buffer. The absorption band of Atto565 dye overlaps with the QD emission, enabling FRET if the two moieties are brought into close contact. The psQDbiotin demonstrated a reduction in the QD fluorescence emission of $15 \%$ not observed with the control psQD as well as an $18 \%$ increase in the sensitized emission of the Atto565.

Dynabeads M-280 Streptavidin (Life Technologies) are micrometer sized negatively charged magnetic beads that present a monolayer of streptavidin on their surface. By applying a magnet all psQDs bound to the magnetic beads can be selectively separated from the supernatant. A solution of psQDs was mixed with different proportions of Dynabeads, all samples were diluted to the same final concentration of $\mathrm{psQD}$, to test whether the $\mathrm{psQD}$ presented accessible biotins. After $30 \mathrm{~min}$ of incubation the Dynabeads were pulled down with a separation magnet and the supernatant fluorescence was measured.
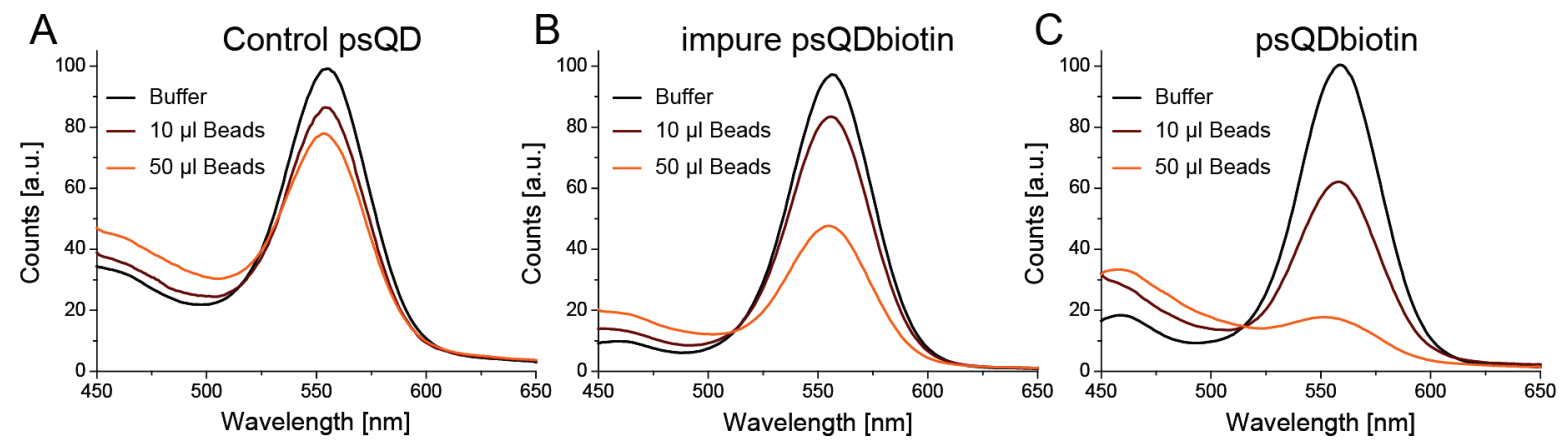

Figure 6: Fluorescence spectra of different psQDs solutions with either buffer, $10 \mu \mathrm{l}$, or $50 \mu \mathrm{l}$ of dynabeads added to the solution. A seperation magnet was applied to all the samples and the supernatent measured, $400 \mathrm{~nm}$ excitation. A) Control psQD B) psQDbiotin which was not purified after preperation. The impurification consists of empty polymersomes and single strand polymer. C) psQDbiotin, the same sample as from B after the purification process.

Though there is some unspecific binding of psQDs to the Dynabeads it was evident that the psQDbiotin bound more efficiently. The binding capacity of the Dynabeads depends on the size and charge of the biotinylated moiety, for example the Dynabead's binding capacity is reduced by 2 orders of magnitude when going from free biotin to a biotinylated antibody. According to the manufacturer the 10 and $50 \mu \mathrm{l}$ of beads added to the solutions should bind approximately 20 and 100 picomoles of 
biotinylated peptides, respectively. The total amount of psQDbiotin in the initial solution was estimated at 5 picomoles. Despite the fact that the psQD is much larger than a peptide and negatively charged with $50 \mu \mathrm{l}$ of beads almost all fluorescence was removed from the supernatant in the psQDbiotin sample, it appears that the biotin was readily available for streptavidin binding.

Figure 6B shows the results of a solution of psQDbiotin which was never purified after preparation, it was contaminated by excess polymer which naturally also had conjugated biotin. The results from Figure 6 are summarized in Table 3. It was observed that the $10 \mu 1$ measurements are equivalent in the control and impure solutions and that both the 10 and $50 \mu$ pull-downs were considerably smaller for the impure solution than for the pure psQDbiotin. The individual polymer strands should exhibit reduced steric hindrance, as compared to the psQDbiotin, and would be expected to bind more readily to streptavidin. It appears that only after removing the excess polymer from the solution was it possible for the Dynabeads to bind the psQDbiotin. The Dynabeads can confirm the presence of accessible biotins on the surface, but a quantitative biotin value cannot be obtained with this strategy.

Table 3: Supernatant fluorescence after Dynabeads separation. ${ }^{\mathrm{a}}$

\begin{tabular}{lccc}
\hline Dynabead volume & Control psQD & Impure psQDbiotin & psQDbiotin \\
\hline $0 \mu \mathrm{l}$ & 100 & 100 & 100 \\
$10 \mu \mathrm{l}$ & 87 & 87 & 61 \\
$50 \mu \mathrm{l}$ & 77 & 49 & 17 \\
\hline
\end{tabular}

[a] Values normalized to initial fluorescence. Excitation wavelength, $400 \mathrm{~nm}$.

\subsubsection{Imaging Experiments with psQDbiotin}

The psQDbiotin preparations were imaged on ACP-EGFR-CHO cells, which had approximately 300,000 EGFR and were biotinylated using the CoA-Biotin and ACP synthase enzyme(see also section 5.2). ${ }^{13}$ After washing, the cells were incubated with $100 \mathrm{nM}$ streptavidin at RT for $15 \mathrm{~min}$. Since no EGF was added, most of the EGFR bound streptavidin should have remained on the membrane with minimal internalization. After another washing step the targeted fluorophore was incubated (1-10 $\mathrm{nM})$ for $20 \mathrm{~min}$ at RT.

Positive controls using biocytin- 488 showed that the $\mathrm{CHO}$ cells were modified as expected, as a specific fluorescent signal could be observed on the cell membrane. Negative controls with bare psQDs showed no unspecific binding. Unfortunately, imaging attempts with the psQDbiotin were unsuccessful. No specific labeling was achieved.

Two possible explanations come to mind. The first series of experiments were conducted with an impure fraction of the psQDbiotin preparation. As was seen in the Dynabead experiments (previous Section), excess single strand polymer containing biotin was much more efficient at binding 
streptavidin than the psQDbiotin, presumably because of better accessibility of the biotin binding pocket of the protein due to steric hindrance of the nanoparticle. As the labeling experiments were realized with $\mathrm{nM}$ concentration of $\mathrm{psQDs}$ there was more than enough free polymer to have saturated the cellular binding sites with the polymer. A second proposed explanation is the electrostatic repulsion of the negatively charged psQDbiotin and the cellular membrane. ${ }^{17}$ In the constructs imaged in Section 5.2 the streptavidin was bound to the psQD and then incubated with the cell, in the experiments in this section the streptavidin was bound directly to the cell. The streptavidin on the psQD surface may act as shielding agents allowing the construct to approach the cellular plasma membrane; comparatively the streptavidin is only a very small fraction of the cell membrane and does not provide efficient shielding. It is therefore possible that a steric-electrostatic repulsion is inhibiting the psQDbiotin from binding with the streptavidin. In the case of commercial QDs, these are normally covered by multiple large PEG groups which provide colloidal stability while shielding the negative charge. Future experiments can be realized with a preformed psQDbiotin in combination with free streptavidin, though care must be taken to avoid unintended crosslinking and aggregation.

\subsection{Conclusions}

For real biological applications the development of targeted psQDs is a necessity. To this end both post-coating and polymer functionalization may find their niche. Post-coating functionalization will normally provide a smaller construct, but has a reduced yield and is more time consuming. Extended linkers should be incorporated to provide the required distance from the psQD surface, avoiding the deactivation of the ligand due to steric hindrance observed in the psQD-EGF construct. Polymer functionalization can be scaled-up in a simpler manner, but considering that the polymer is prepared in 100\% organic solvents most complex biological molecules will be excluded as possibilities. The biotin functionalized polymer provides a pathway for producing large quantities of psQDs that can later be combined with streptavidin and a biotinylated ligand for targeting.

The inclusion of additional PEG moieties on the psQD exterior should be investigated. PEG not only increases the QY of water soluble QDs, but also avoids protein corona formation and shields the negative surface charge of polymer coated NPs. ${ }^{18}$ The increase in QY is very important as the localization precision of single emitters depends on the amount of photons obtained from the emitter. Along with other variables such as extended linkers for the biotin, the addition of PEG may be a solution to the inefficient cellular labeling. The addition of PEGs to the polymer coating may also reduce the toxicity of the cadmium containing QDs. ${ }^{18}$

To this point the psQD excitation source has been $405 \mathrm{~nm}$ diode laser as this wavelength is an absorption minimum of both the closed and open form of the PC, and should therefore minimize photoconversion. Other excitation options may be utilized but they should be examined to assure that they are not involuntarily modifying the psQD photostate. The conditions for photoswitching beyond 
ensemble measurements in simple alkaline buffers must be optimized. As observed in the single particle experiments in Chapter 4, under intense light sources in oxidative conditions the psQDs are readily bleached. Different light sources, intensities, and irradiation times can be tested to optimize the emission modulation while avoiding bleaching the QD or causing photodamage to the cell.

\section{References}

1. Bublil, E. M.; Yarden, Y. The Egf Receptor Family: Spearheading a Merger of Signaling and Therapeutics. Curr Opin Cell Biol 2007, 19, 124-134.

2. Mendelsohn, J. Targeting the Epidermal Growth Factor Receptor for Cancer Therapy. J Clin Oncol 2002, 20, 1S-13S.

3. Yarden, Y.; Schlessinger, J. Epidermal Growth Factor Induces Rapid, Reversible Aggregation of the Purified Epidermal Growth Factor Receptor. Biochem. 1987, 26, 1443-1451.

4. Oda, K.; Matsuoka, Y.; Funahashi, A.; Kitano, H. A Comprehensive Pathway Map of Epidermal Growth Factor Receptor Signaling. Mol Syst Biol 2005, 1, 20050010.

5. Lidke, D. S.; Nagy, P.; Heintzmann, R.; Arndt-Jovin, D. J.; Post, J. N.; Grecco, H. E.; Jares-Erijman, E. A.; Jovin, T. M. Quantum Dot Ligands Provide New Insights into ErbB/Her Receptor-Mediated Signal Transduction. Nat. Biotechnol. 2004, 22, 198-203.

6. Lidke, D. S.; Lidke, K. A.; Rieger, B.; Jovin, T. M.; Arndt-Jovin, D. J. Reaching out for Signals: Filopodia Sense EGF and Respond by Directed Retrograde Transport of Activated Receptors. J. Cell Biol. 2005, 170, 619-626.

7. Sperling, R. A.; Parak, W. J. Surface Modification, Functionalization and Bioconjugation of Colloidal Inorganic Nanoparticles. Philos. Trans. R. Soc. A 2010, 368, 1333-1383.

8. Hermanson, G. T., Bioconjugate Techniques. Academic Press, Inc., : San Diego, USA., 1996.

9. Diaz, S. A.; Toscani, A. M.; Arndt-Jovin, D. J.; Jovin, T. M.; Jares-Erijman, E. A. In Small Photostable Photoswitchable Quantum Dots as Nanotools for Live Cell Imaging, NSTI-Nanotech 2011,2011; Boston, MA; United States, pp 205-208.

10. Nexø, E.; Frede Hansen, H. Binding of Epidermal Growth Factor from Man, Rat and Mouse to the Human Epidermal Growth Factor Receptor. Biochim. Biophys. Acta - General Subjects 1985, 843, 101106.

11. Hyre, D. E.; Le Trong, I.; Merritt, E. A.; Eccleston, J. F.; Green, N. M.; Stenkamp, R. E.; Stayton, P. S. Cooperative Hydrogen Bond Interactions in the Streptavidin-Biotin System. Protein Sci. 2006, 15, 459467.

12. Nagy, P.; Arndt-Jovin, D. J.; Jovin, T. M. Small Interfering RNAs Suppress the Expression of Endogenous and GFP-Fused Epidermal Growth Factor Receptor (ErbB1) and Induce Apoptosis in ErbB1-Overexpressing Cells. Exp Cell Res 2003, 285, 39-49.

13. George, N.; Pick, H.; Vogel, H.; Johnsson, N.; Johnsson, K. Specific Labeling of Cell Surface Proteins with Chemically Diverse Compounds. J. Am. Chem. Soc. 2004, 126, 8896-8897.

14. Johnsson, N.; George, N.; Johnsson, K. Protein Chemistry on the Surface of Living Cells. ChemBioChem 2005, 6, 47-52. 
15. De Beule, P. A. A.; de Vries, A. H. B.; Arndt-Jovin, D. J.; Jovin, T. M., Generation-3 Programmable Array Microscope (PAM) with Digital Micro-Mirror Device (DMD). In Emerging Digital Micromirror Device Based Systems and Applications Iii, Douglass, M. R.; Oden, P. I., Eds. Spie-Int Soc Optical Engineering: Bellingham, 2011; Vol. 7932.

16. Díaz, S. A.; Giordano, L.; Jovin, T. M.; Jares-Erijman, E. A. Modulation of a Photoswitchable DualColor Quantum Dot Containing a Photochromic FRET Acceptor and an Internal Standard. Nano Lett. 2012, 12, 3537-3544.

17. Hühn, D.; Kantner, K.; Geidel, C.; Brandholt, S.; De Cock, I.; Soenen, S. J. H.; Rivera_Gil, P.; Montenegro, J.-M.; Braeckmans, K.; Müllen, K.; Nienhaus, G. U.; Klapper, M.; Parak, W. J. PolymerCoated Nanoparticles Interacting with Proteins and Cells: Focusing on the Sign of the Net Charge. ACS Nano 2013, 7, 3253-3263.

18. Walling, M. A.; Novak, J. A.; Shepard, J. R. Quantum Dots for Live Cell and in Vivo Imaging. Int J Mol Sci 2009, 10, 441-491. 


\section{CHAPTER 6: SUMMARY \& OUTLOOK}

The work presented in this thesis integrates many diverse fields of research such as chemistry, nanotechnology, photophysics, and biology. The end result is the preparation, characterization, and deployment of the first reported water soluble photoswitchable QD. The chemical aspects of the work focused on the synthesis of various PCs and comb-like amphiphilic photochromic polymers. The photochromic polymers were then studied in different solvents alone or in combination with QDs. The self-assembly of these systems created different nanoprobes with varying properties and characteristics. For example, the combination of the polymer and QDs in buffer solution created the aforementioned psQDs, the properties of which were investigated in ensemble and single particle experiments with the aim of obtaining a complete photophysical description. The psQDs were further modified to target live cells and fluorescence microscopy experiments were carried out as a preliminary study of their potential biological applications.

The properties of QDs as fluorophores have been extolled throughout this thesis and I have presented psQDs as a possible next step in the use of QDs in microscopy, combining the advantages of traditional QDs with an added photomodulation capacity. The comprehensive description of psQDs from preparation to application - provides insights for the rational design of improved future probes.

\section{Chemistry}

A nucleophilic acyl substitution of the PC's aromatic amine on an acyl chloride, was adopted as a simple and economic method for synthesizing asymmetric mono-functionalized PCs with a primary amine available for further modification. The basic PC was modified with alkyl linkers of different lengths, which in turn were conjugated to PMA to create variations of the photoswitchable polymers. The primary amine on the end of the linker can readily react with a multitude of reagents, allowing for specific functionalizations, as was seen with the synthesis of the UV activated crosslinking PC. The photochemical properties of the synthesized PCs, such as photocyclization rate and efficiency, were studied and it was determined that their conjugation through an aliphatic linker to the polymer does not drastically modify their photochemical properties.

Multiple photochromic amphiphilic comb polymers were synthesized and characterized. The protocol reported in the literature was modified into a two-step addition of dodecylamine and adjustment of the initial solvent to consider dye solubility; the new protocol makes the conjugation of amine containing dyes more efficient and reproducible. The addition of multiple functional moieties, including hydrophilic dyes (Alexa647 and lucifer yellow) and biological molecules (biotin), was also explored. A standard protocol for characterizing PMA based polymers using absorbance and NMR spectroscopy was developed and applied successfully. The absorbance contribution of each component could be separated and quantified with absorbance spectroscopy; in the case of the PC it was the 
change in absorbance upon UV irradiation that allowed the determination of PC yield. Molecules with low extinction coefficients were quantified with ${ }^{1} \mathrm{H}$ NMR spectroscopy; as an example, the integration of the 3.65 ppm peak was used to quantify the concentration of PEG linkers.

Based on the protocols presented in this thesis new pcFRET pairs as well as multi-functional polymers for coating NPs can be conceptualized, synthesized, characterized, and exploited experimentally.

\section{Nano-Conformations}

The design and preparation of an amphiphilic comb-like polymer containing the pcFRET pair of $\mathrm{PCalaNH}_{2}$ and $\mathrm{LY}$ allowed the interrogation of the self-assembly conformations adopted by the polymer.

Through the use of polymer chain theory and fluorescence lifetime anisotropy it was determined that in organic solvents the polymers remain as individual strands which are well described as freely draining dynamic chains with a $4 \mathrm{~nm}$ diameter.

The multistrand empty polymersomes formed by the polymer in alkaline buffer were also characterized: size ( $75 \mathrm{~nm}$ diameter) and emission photomodulation ( $20 \%$ in steady-state, $13 \%$ in lifetime fluorescence). It was confirmed that voluminous dyes affect the hydrophobic nature of the polymersome interior as well as negatively affecting the NP coating efficiency. Dilution of the original polymersome with a dye-free polymer resulted in the increase of the photomodulation in both the steady-state and lifetime measurements, $30 \%$ and $21 \%$ respectively, as a result of the increased $(\sim 3-$ fold) PC photoconversion in the more hydrophobic environment.

In the presence of NPs with organic surface ligands the polymer will remain independent in organic solvents, but can coat the NPs and transfer them to aqueous medium when resuspended in alkaline buffers. It was confirmed that polymer conjugated hydrophobic moieties, such as the PCs, preferably occupy the hydrophobic microenvironment formed by the QD and the polymer coat. The intermediate environment could be adequately described as chloroform-like; both the QD and PC sensed it as such. The PC photoconversion was similar in both media and the polymer capped QD had the same QY as one suspended in $\mathrm{CHCl}_{3}$. QDs were utilized as templates to create small $(\sim 20 \mathrm{~nm}$ diameter) photomodulatable fluorescent probes. By templating multiple polymer strands around a 635 $\mathrm{nm}$ emitting QD the photomodulation properties of the probe were drastically increased $(69 \%$ and $56 \%$ in steady-state and lifetime determinations respectively) while creating a dual-color photoswitchable fluorescent nanoparticle. 


\section{Photoswitchable Quantum Dots}

Photochromic amphiphilic comb-like polymers were utilized to develop the first water soluble photoswitchable QD. The psQDs are small ( $\sim 15 \mathrm{~nm}$ hydrodynamic diameter) probes that can undergo multiple on-off cycles without fatigue in buffer solution with an emission quenching of up to $50 \%$ in steady state and $47 \%$ in fluorescence lifetime determinations. Versions of the psQDs were prepared with a secondary Alexa647 dye, which was conjugated to the polymer before coating and acted as an internal reference, thereby providing the construct with ratiometric properties. The QD-Alexa647 emission ratio could be modified nearly $100 \%$ by the photoconversion of the PCs. A very useful feature of all the psQD preparations was the prominent quenching of the QD exerted by low degrees of photoconversion obtained at short $(1 \mathrm{sec})$ irradiation times.

A model was proposed to explain the complex PC photoconversion and QD emission quenching kinetics of the psQDs. The critical concepts of the model are i) the existence of a heterogeneous set of PCs around the QD donor, that can be represented by 2 classes of molecules differing only in FRET efficiency, and ii) that during UV irradiation the PC photoreversal $(\mathrm{cPC} \rightarrow \mathrm{oPC})$ due to QD-cPC FRET is considerable and depends on the FRET efficiency. The Class 1 acceptors are very efficient and account for the high quenching at low acceptor concentrations although being constrained to low $\alpha_{\mathrm{PS}}$ values due to the FRET-induced photoreversal. The Class 2 acceptors dominate the cPC conversion time course but are not very efficient in FRET, accounting for the asymptotic tail observed in the data. The Class $2 \alpha_{\mathrm{PS}}$ is also reduced but comparatively very little. The representation was the simplest proposed model that sufficed for fitting the data for every psQD regardless of the type of QD or polymer used in the preparation.

psQDs were prepared in which the average PC-QD distance was varied by changing the length of the PC's linker. Initial experiments indicated that there is a limit to the QD quenching capacity $(<50 \%)$. This limitation was predicted by the above mentioned model; as the efficiency of the individual FRET pairs (QD-cPC) increases the total number of PC in the acceptor form is diminished, eventually reaching an equilibrium state. I hypothesize that the latter is determined by the selected pcFRET pair and irradiation conditions, as these will modify the $\mathrm{R}_{\mathrm{o}}$ and $\alpha_{\mathrm{PS}}$. This hypothesis predicts that there is an upper limit to the maximum attainable quenching, and it is independent of the D-A distance and the total number of PCs placed around the QD so long as the QD absorbance predominates over the oPC absorbance. Future experiments using psQDs with different linker lengths on the PC as well as polymers that contained mixed PCs should provide additional insight.

To further comprehend the psQD photophysics a series of single particle microscopy (SPM) experiments were conceptualized and realized in conjunction with collaborators at the Univ. of Twente (Enschede, The Netherlands). The psQDs could be detected at the single particle level in both laser scanning confocal and TIRF microscopes and the ensemble photochromic end states of the psQDs 
were successfully characterized. The SPM supports a model according to which the photocyclization into the PS state causes a fraction of the psQDs to go into a long-lived dark state. The emitting psQDs in the PS state cannot be distinguished from the psQDs in the open state, as they present the same intensity, blinking statistics, and ON/OFF ratios. A 50\% decrease in ensemble emission would therefore correlate with 1 in 2 psQDs ceasing to emit and not a general dimming of all psQDs. The experimental conditions for detection of single particle photoswitching have as yet not been ascertained, although previous investigations will simplify the selection of the imaging medium as well as the duration and intensity of irradiation. These experiments must be realized before the extended dark state hypothesis can be confirmed.

Biological targeting of psQDs was achieved through both a post-coating NP functionalization and a pre-coating polymer functionalization strategy. The post-coating methodology allows for the downstream modification of any $\mathrm{psQD}$ for a specific use, but the conjugation and purification is intricate and results in lower yields of the desired construct. psQD-streptavidin constructs were targeted to the EGFR of live cells and some initial photomodulation results ( $25 \%$ intensity) were obtained.

Polymer functionalization was realized with a PEG-biotin moiety. While the number of biological molecules that can withstand the polymer conjugation in organic solvents is limited, the advantage of pre-coating strategies is that they can be readily scaled up. Biotin is an adaptable molecule which in combination with streptavidin and a biotinylated ligand allows for specific targeting. The psQDbiotin nanoparticles were successfully bound to streptavidin coated magnetic nanoparticles, demonstrating the availability of the surface biotins.

\section{Outlook}

The field of fluorescent probe development is experiencing a resurgence as fluorescence microscopy continues to grow. The work presented in this thesis will provide an additional steppingstone to new and improved probes, even beyond the featured psQDs. For psQDs to achieve their potential as probes it will be necessary to complete their characterization. For example, single-particle photoswitching must succeed in order to test the extended dark-state hypothesis. The conditions for live cell microscopy also require troubleshooting in order to obtain complete and consistent labeling with psQDs as well as photomodulation, preferably with existing microscope components. If this can be achieved psQDs may find widespread use, particularly for STORM and OLID microscopy.

The submitted pcFRET model may be refined by studying psQDs with varying PC linker lengths. The data obtained from these experiments will help to further comprehend the observed limitation of quenching, which in turn will facilitate the design of more efficient photomodulating probes. The pcFRET model may have applications to systems beyond fluorescent nanoprobes. The 
design of photoswitchable polymers, for detectors or optical data storage, may be improved by modifying the selected pcFRET pair and their relative concentrations based on calculations derived from the proposed pcFRET model.

The experience I gained throughout this thesis leads me to believe that the final, or ideal, psQDs will differ substantially from the ones reported here. The ideal psQD for fluorescence microscopy will have the following traits. The selected QD will emit much farther in the red as red shifted QDs have better QY and higher molar absorptivity, and cellular microscopy is more feasible (NIR water window) and efficient at that wavelength. The polymer used to coat the QDs will most probably have completely different photochromes. Ideally the photochrome will have a high extinction coefficient and a $\alpha_{P S}$ close to unity. The photochromes may even present fluorescence emissions in a different spectral region than the QD. The polymer will include multiple PEGs to ensure that the QY of the QD is not adversely affected and multiple functional moieties, such as biotin, to allow for biological targeting. Within the scope of this thesis some of these objectives were tested, including the coating of red emitting QDs and the synthesis of polymers with PEG and biotin able to efficiently coat QDs. The present limitation to the development of this ideal psQD is the lack of small, efficient photoconverting, thermally stable, fatigue resistant, red-shifted photochromes. It can be anticipated that these will become available as a result of the intense current research activity in this field. 


\section{CHAPTER 7: EXPERIMENTAL}

All reagents and solvents were used as received. Reagents and solvents were acquired from SigmaAldrich unless specifically noted. When anhydrous or dry solvents were required analytical grade solvents were used and were dried with $4 \AA$ activated molecular sieves.

\subsection{Synthesis}

\subsubsection{Synthesis of 3-iodo-2-methylbenzo[b]thiophene}<smiles>Cc1sc2ccccc2c1I</smiles>

2-methylbenzo[b]thiophene ( $2.0 \mathrm{~g}, 14$ mmoles) was prepared in $50 \mathrm{ml}$ acetic acid, $10 \mathrm{ml}$ $\mathrm{H}_{2} \mathrm{O}, 2 \mathrm{ml}$ concentrated $\mathrm{H}_{2} \mathrm{SO}_{4}$. At RT and under vigorous stirring, $\mathrm{I}_{2}$ (2.1 g, 8.4 mmoles) and $\mathrm{H}_{5} \mathrm{IO}_{6}(0.8 \mathrm{~g}, 3.5$ mmoles $)$ were added and the reaction proceeded for 3 hours. The reaction was quenched with $\mathrm{NaHSO}_{3}$ and $\mathrm{NaOH}$. The crude reaction was extracted with ethyl acetate, and the organic phase was washed with brine, dried with $\mathrm{Na}_{2} \mathrm{SO}_{4}$, filtered, and evaporated in a Rotovap system. The crude product is dissolved in $25 \mathrm{ml}$ of $\mathrm{MeOH}$ in reflux, and then drops of water are added until a cloudy white solution is observed. The flask is removed from heating and placed in an ice bath for 90 minutes. The white crystals are filtered to obtain the final product $\left(\mathrm{R}_{\mathrm{f}}\right.$ $=0.62 \mathrm{n}$-heptane; $3.45 \mathrm{~g}$, yield: 90\%). ${ }^{1} \mathrm{H}$ NMR $\left(400 \mathrm{MHz}, \mathrm{CDCl}_{3}\right) \delta 2.60(\mathrm{~s}, 3 \mathrm{H}, \mathrm{CH} 3), 7.32(\mathrm{t}, 1 \mathrm{H}, \mathrm{H}-$ 6), $7.41(\mathrm{t}, 1 \mathrm{H}, \mathrm{H}-5), 7.66$ (d, 1H, H-4), 7.71 (d, 1H, H-7). ${ }^{1,2}$

\subsubsection{Synthesis of 3,3'-(3,3,4,4,5,5-hexafluorocyclopent-1-ene-1, 2-diyl)bis(2- methylbenzo[b]thiophene)}

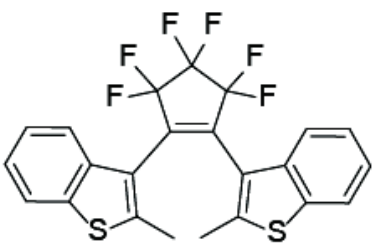

$\mathrm{PC}$

A solution of $n$-BuLi (1.6M in hexane, $2.22 \mathrm{~mL})$ was added to a solution of 3-iodo-2-methylbenzo[b]thiophene $(1,0 \mathrm{~g}, 3.65 \mathrm{mmoles})$ in $15 \mathrm{~mL}$ of anhydrous THF at $-78{ }^{\circ} \mathrm{C}$ under an inert Argon atmosphere. The solution was stirred for one hour, and a white precipitate was observed. At this point $0.25 \mathrm{~mL}$ of perfluorocyclopent-1-ene (Zeon Chemicals L.P., $\delta=1.6 \mathrm{~g} \mathrm{~cm}^{-3}$ ) was added. The solution turned yellow and after one hour at $-78{ }^{\circ} \mathrm{C}$ was allowed to reach room temperature. The solution was neutralized with $30 \mathrm{~mL}$ of $1 \mathrm{~N} \mathrm{HCl}$. The crude reaction was extracted with ethyl acetate, and the organic phase was washed with brine, dried with $\mathrm{Na}_{2} \mathrm{SO}_{4}$, filtered, and evaporated in a Rotovap system. Purification was performed on a silica gel column with cyclohexane as solvent, with the final product obtained as a white solid $\left(\mathrm{R}_{\mathrm{f}}=0.40 \mathrm{n}\right.$-heptane; $0.48 \mathrm{~g}$, yield: $\left.57 \%\right)$. ${ }^{1} \mathrm{H}$ NMR (400MHz, $\mathrm{CDCl}_{3}$ ) $\delta 2.2$ (s, 6H, CH3 ap), 2.49 (s, 3H, CH3 p), 7.17-7.20 (dd, 2H, H-5/H-6 p), 7.30 (m, 2H, H-6 ap), 7.38 (m, 2H, H-5 ap), 7.55-7.61 (m, 2H, H-4/H-7 p), 7.68 (m, 4H, H-4/H-7 ap). Parallel (p) to antiparallel (ap) conformers $35: 65{ }^{1}$ 


\subsubsection{Synthesis of 2-methyl-3-(perfluorocyclopent-1-enyl)benzo[b]thiophene}

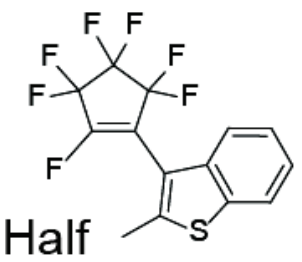

A solution of $n$-BuLi (1.6M in hexane, $2.22 \mathrm{~mL})$ was added to a solution of 3iodo-2-methylbenzo[b]thiophene ( $1 \mathrm{~g}, 3.65 \mathrm{mmoles})$ in $15 \mathrm{~mL}$ of anhydrous THF at $-78{ }^{\circ} \mathrm{C}$ under an inert Argon atmosphere. The solution was stirred for one hour, and a white precipitate was observed. At this point $0.13 \mathrm{~mL}$ of perfluorocyclopent-1-ene was added. The solution turned yellow and after one hour at $-78{ }^{\circ} \mathrm{C}$ was allowed to reach room temperature. The solution was neutralized with $30 \mathrm{~mL}$ of $1 \mathrm{~N} \mathrm{HCl}$. The crude reaction was extracted with ethyl acetate $(2 \times 20 \mathrm{~mL})$, and the organic phase was washed with brine, dried with $\mathrm{Na}_{2} \mathrm{SO}_{4}$, filtered, and evaporated in a Rotovap system. Purification was performed on a silica gel column with cyclohexane as solvent, with the final product obtained as a white solid $\left(\mathrm{R}_{\mathrm{f}}=\right.$ 0.70 n-heptane; 0.93 g, yield: $75 \%) .{ }^{1} \mathrm{H}$ NMR $\left(400 \mathrm{MHz}, \mathrm{CDCl}_{3}\right) \delta 2.53(\mathrm{~s}, 3 \mathrm{H}, \mathrm{CH} 3), 7.36(\mathrm{~m}, 1 \mathrm{H}, \mathrm{H}-$ 6), 7.40 (m, 1H, H-5), 7.49 (d, 1H, H-4), 7.80 (dd, 1H, H-7). ${ }^{1,3}$

\subsubsection{Synthesis of [3-(3,3,4,4,5,5-Hexafluoro-2-(2-methylbenzo[b]thiophen- 3- yl)cyclopent-1-en-1-yl)-2-methyl-6-nitrobenzo[b]thiophene]}

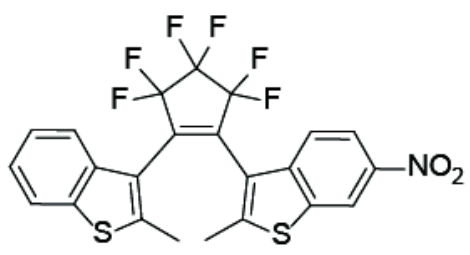

$\mathrm{PCmNO}_{2}$

PC (89 mg, $190.2 \mu$ moles) was added to $2.3 \mathrm{~mL}$ of glacial acetic acid and $0.3 \mathrm{~mL}$ of acetic anhydride in an ice bath. Then $0.1 \mathrm{~mL}$ of fuming nitric acid was slowly added; the solution was mixed vigorously and maintained at $\sim 5{ }^{\circ} \mathrm{C}$. After $15 \min$ the temperature was raised to 10 ${ }^{\circ} \mathrm{C}$, and then the solution was allowed to react overnight at RT. The reaction was quenched by the addition of $5 \mathrm{~mL}$ of water, at which point a white-yellow precipitate was observed. The solution was neutralized with concentrated aqueous $\mathrm{NaOH}$. The crude reaction was extracted with ethyl acetate, and the organic phase was washed with water, dried with $\mathrm{Na}_{2} \mathrm{SO}_{4}$, filtered, and evaporated in a Rotovap system. Purification was performed on a silica gel column with cyclohexane-ethyl acetate (98:2) as solvent, with a final product a white-yellow solid $\left(\mathrm{R}_{\mathrm{f}}=0.40,97: 3\right.$ cyclohexane: ethyl acetate; $38.2 \mathrm{mg}$, yield: $39 \%) .{ }^{1} \mathrm{H}$ NMR (400MHz, $\left.\mathrm{CDCl}_{3}\right) \delta 2.21$ (s, 2H, CH3 ap), 2.31 (s, 2H, CH3 ap), 2.49 (s, 1H, CH3 p), 2.58 (s, 1H, CH3 p), 7.20-7.76 (m, 5H, ArH), 8.10 (d, 0.4H, H-7 p), 8.26 (d, 0.6H, H-7 ap), 8.54 (s, 0.4H, H-7 p), 8.64 (s, 0.6H, H-7 ap). [p:ap] conformers $40: 60 .^{4}$

\subsubsection{Synthesis of [3,3'-(perfluorocyclopent-1-ene-1,2-diyl)bis(2-methyl-6- nitrobenzo[b]thiophene)]}

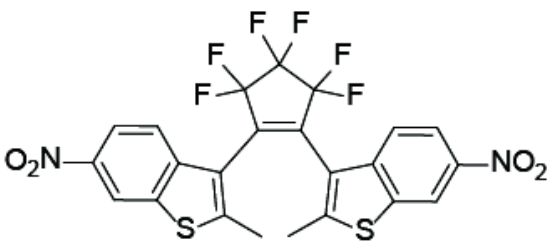

PC (89 mg, $190.2 \mu$ moles) was added to $2.3 \mathrm{~mL}$ of glacial acetic acid and $0.3 \mathrm{~mL}$ of acetic anhydride in an ice bath. Then 0.15 $\mathrm{mL}$ of fuming nitric acid was slowly added; meanwhile the solution was mixed vigorously and maintained at $\sim 10-15{ }^{\circ} \mathrm{C}$.

After 15 min the solution was allowed to reach RT and react for 24 hs. The reaction was quenched by the addition of $5 \mathrm{~mL}$ of water, at which point a yellow precipitate was observed. The solution was 
neutralized with concentrated aqueous $\mathrm{NaOH}$. The crude reaction was extracted with ethyl acetate, and the organic phase was washed with water, dried with $\mathrm{Na}_{2} \mathrm{SO}_{4}$, filtered, and evaporated in a Rotovap system. Purification was performed on a silica gel column with cyclohexane-ethyl acetate $(98: 2)$ as solvent, with a final product a light yellow solid $\left(\mathrm{R}_{\mathrm{f}}=0.20,97: 3\right.$ cyclohexane: ethyl acetate; $49.1 \mathrm{mg}$, yield: 46\%). ${ }^{1} \mathrm{H}$ NMR (400MHz, $\left.\mathrm{CDCl}_{3}\right) \delta 2.53$ (s, 3.6H, CH3 ap), 2.59 (s, 2.4H, CH3 p), $7.62(\mathrm{~d}$, 0.8H, H-5 p), 7.74 (d, 1.2H, H-5 ap), 8.08 (d, 1.2H, H-6 ap), 8.11 (d, 0.8H, H-6 ap), 8.58 (d, 0.8H, H-7 p), 8.67 (d, 1.2H, H-7 ap). [p:ap] conformers 40:60. ${ }^{4}$

\subsubsection{Synthesis of 3-(3,3,4,4,5,5-hexafluoro-2-(2-methylbenzo[b]thiophen-3- yl)cyclopent-1-enyl)-2-methylbenzo[b]thiophen-6-amine}

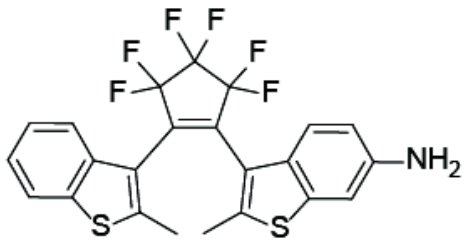

$\mathrm{PCmNH}_{2}$

A solution of $\mathrm{PCmNO}_{2}(24 \mathrm{mg}, 46.7 \mu$ moles $)$ was prepared in $5 \mathrm{~mL}$ of methanol. $\mathrm{NiCl} 2 \cdot 6 \mathrm{H}_{2} \mathrm{O}(86.76 \mathrm{mg})$ was added under vigorous mixing until complete solubility of $\mathrm{PCmNO}_{2}$ was achieved. The solution was placed in an ice bath, and then $\mathrm{NaBH}_{4}(43.5 \mathrm{mg}, 1.14$ mmoles) was slowly added, leading to the immediate formation of a black precipitate. The solution reached completion in $\sim 10 \mathrm{~min}$ at $\mathrm{RT}$, was quenched with $0.04 \mathrm{~N} \mathrm{HCl}$, filtered, and then evaporated in a Rotovap. The residue was extracted with $\mathrm{CH}_{2} \mathrm{Cl}_{2}$, and the organic phase was washed with water, dried with $\mathrm{Na}_{2} \mathrm{SO}_{4}$, filtered, and evaporated in a Rotovap system. Purification realized on a silica column $(100 \%$ cyclohexane with gradual increase of ethyl acetate until 90:10 is reached). $\mathrm{PCmNH}_{2}$ was obtained as a pink oil $\left(\mathrm{R}_{\mathrm{f}}=0.25,70: 30\right.$ cyclohexane: ethyl acetate; $16.9 \mathrm{mg}$, yield: $75 \%) .{ }^{1} \mathrm{H}$ NMR $\left(400 \mathrm{MHz}, \mathrm{CDCl}_{3}\right) \delta 2.10$ (s, $\left.2.9 \mathrm{H}, \mathrm{CH} 3 \mathrm{ap}\right), 2.21$ (s, 3.1H, CH3 ap) 2.43 (2s, 3.3H, CH3 p), 6.54 (d, 0.4H, H-5 p), 6.77 (d, 0.9H, H-5 ap), 6.87 (s, 0.4H, H-7 p), 6.96 (s, 0.8H, H-7 ap), 7.17-7.77 (m, 9.5H, ArH). [p:ap] conformers 35:65.

\subsubsection{Synthesis of 4-((3-(3,3,4,4,5,5-hexafluoro-2-(2-methylbenzo[b]thiophene-3- yl)cyclopent-1-en-1-yl]-2-methylbenzo[b]thiophene-6-yl)amino)-4-oxobutanoic acid}

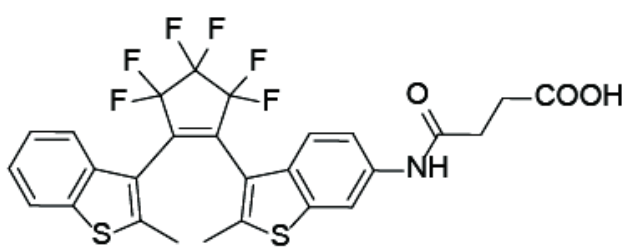

PCmonomer

A solution of succinic anhydride ( $4 \mathrm{mg}, 40.0 \mu$ moles) in anhydrous THF $(1 \mathrm{ml})$ is added to a solution of $\mathrm{PCmNH}_{2}(5$ $\mathrm{mg}, 10.4 \mu$ moles $)$ in $\mathrm{CHCl}_{3}(2 \mathrm{ml})$. The solution was heated to $55{ }^{\circ} \mathrm{C}$ and allowed to react for 3 hours. The reaction was quenched with $0.1 \mathrm{M} \mathrm{NaOH}(2 \mathrm{ml})$ and then extracted with

$\mathrm{CHCl}_{3}$. The organic phase was washed with a minimum amount of water, dried with $\mathrm{Na}_{2} \mathrm{SO}_{4}$, filtered, and evaporated in a rotovap system. The crude product was purified with a preparative thin layer chromatogram using $20 \times 20 \mathrm{~cm}$ normal phase plate and cyclohexane: ethyl acetate (5:3) solvent. The extraction from the stationary phase was realized with DMF. A grey precipitate was obtained $\left(\mathrm{R}_{\mathrm{f}}=\right.$ 0.15, 50:30 cyclohexane: ethyl acetate; $4.7 \mathrm{mg}$, yield: 78\%). ${ }^{1} \mathrm{H}$ NMR (400 MHz, CDCl3) $\delta 2.15$ (s, 1.95H, CH3 ap), 2.18 (s, 1.95H, CH3 ap) 2.46 (2s, 2.1H, CH3 p), 2.70 (t, 2H, H-9,), 2.80 (t, 2H, H-9), 6.98-7.70 (m, 6H, ArH) 8.16 (s, 0.35H, H-7 p), 8.20 (s, 0.65H, H-7 ap). [p:ap] conformers 35:65. 


\subsubsection{Synthesis of Acyl Chlorides}

A commercially available linker which presented a carboxyl group on one end and a protected amine on the other (205 $\mu$ moles) was added to a flask that had been previously dried and flushed with argon. Thionyl chloride ( $22 \mu 1,300 \mu$ moles, CAS: 7719-09-7) was added, the flask was flushed with argon and the reaction allowed to proceed for $30 \mathrm{~min}$ at $50{ }^{\circ} \mathrm{C}$. The flask was dried in a rotovap system for $60 \mathrm{~min}$ at $50{ }^{\circ} \mathrm{C}$, excess reagent, $\mathrm{SO}_{2}$, and $\mathrm{HCl}$ are therefore eliminated and freshly formed acyl chloride can be used in the next step.

\subsubsection{Synthesis of 2-amino- $\mathrm{N}-(3-(3,3,4,4,5,5$-hexafluoro-2-(2-methylbenzo[b]thiophen-3- yl)cyclopent-1-enyl)-2-methylbenzo[b]thiophen-6-yl)propanamide}

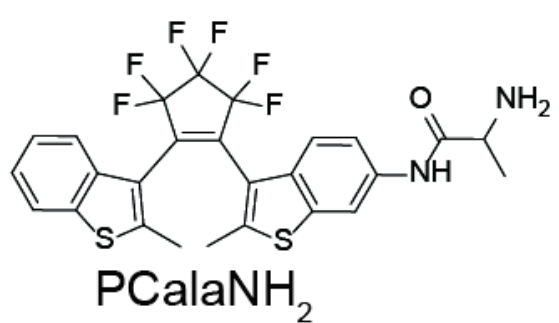

A solution containing $\mathrm{PCmNH}_{2}(48 \mathrm{mg}, 100 \mu$ moles $)$ was dissolved in dry $\mathrm{CHCl}_{3}$. The solution was added to a dry flask containing (9H-fluoren-9-yl)methyl 1-chloro-1-oxopropan-2ylcarbamate (59 mg, $180 \mu$ moles) and allowed to react at $30^{\circ} \mathrm{C}$ for $2 \mathrm{~h}$. The reaction was neutralized with $\mathrm{NaOH}$ and the crude product was extracted with $\mathrm{CH}_{2} \mathrm{Cl}_{2}$; a pink oil was obtained. Purification was performed on a silica gel column with cyclohexane: ethyl acetate (70:30) mobile phase. The intermediate product (9H-fluoren9-yl)methyl 1-(3-(3,3,4,4,5,5-hexafluoro-2-(2-methylbenzo[b]thiophen-3-yl)cyclopent-1-enyl)-2methylbenzo[b]thiophen-6-ylamino)-1-oxopropan-2-ylcarbamate, PCalaFmoc was obtained (64 mg, $84 \mu$ moles $)$ as a red foam. $\left(\mathrm{R}_{\mathrm{f}}=0.30,70: 30\right.$ cyclohexane: ethyl acetate $)$

The Fmoc protective group was released from the aliphatic amine by addition of excess piperidine (650 $\mu 1,6.5$ mmoles) in $\mathrm{CHCl}_{3}$ at $50{ }^{\circ} \mathrm{C}$ for 20 minutes. The reaction was quenched with $\mathrm{HCl}$ and extracted with $\mathrm{CH}_{2} \mathrm{Cl}_{2}$. The product was purified by silica gel column, with cyclohexane: THF (30:70) mobile phase. Solvent was evaporated in a rotovap system and the final product was obtained as a yellow/brown oil $\left(\mathrm{R}_{\mathrm{f}}=0.0,70: 30\right.$ cyclohexane: ethyl acetate; $41.5 \mathrm{mg}$, yield: $\left.75 \%\right)$. ${ }^{1} \mathrm{H}$ NMR (400 $\mathrm{MHz}, \mathrm{CDCl}_{3}$ ) $\delta 1.27$ (d, 1.05H, CH3 p), 1.29 (s, 1.25H, CH3), 2.17 (s, 1.20H, Ar-CH3 ap), 2.20 (s, 1.75H, Ar-CH3 ap), 2.48 (2s, 2.40H, Ar-CH3 p), 3.65 (m, 1.07, CH), 6.80-7.70 (m, 6H, ArH), 8.31 (s, 0.40H, H-7 ap), 8.35 (s, 0.20H, H-7 p), 9.53 (s, 0.30H, Ar-NH-CO), 9.65 (s, 0.51H, Ar- $\underline{-}-\mathrm{CO}$ ). [p:ap] conformers 40:60

\subsubsection{Synthesis of 6-amino-N-(3-(3,3,4,4,5,5-hexafluoro-2-(2-methylbenzo[b]thiophen- 3-yl)cyclopent-1-enyl)-2-methylbenzo[b]thiophen-6-yl)hexanamide}

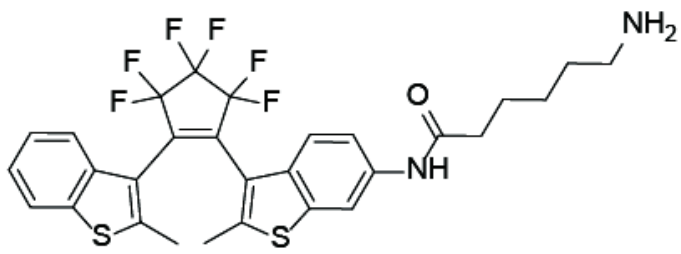

PCahxNH

A solution containing $\mathrm{PCmNH}_{2}(48 \mathrm{mg}, 100 \mu$ moles $)$ was dissolved in dry $\mathrm{CHCl}_{3}$. The solution was added to a dry flask containing tert-butyl 6-chloro-6oxohexylcarbamate (45 mg, $180 \mu$ moles) and allowed to react at $30^{\circ} \mathrm{C}$ for $2 \mathrm{~h}$. The reaction was neutralized with

$\mathrm{NaOH}$ solution and the crude was extracted with $\mathrm{CH}_{2} \mathrm{Cl}_{2}$, a greenish-brownish oil was obtained. 
Purification was performed on a silica gel column with cyclohexane:ethyl acetate (90:10) mobile phase. The intermediate product tert-butyl 6-(3-(3,3,4,4,5,5-hexafluoro-2-(2-methylbenzo[b]thiophen3-yl)cyclopent-1-enyl)-2-methylbenzo[b]thiophen-6-ylamino)-6-oxohexylcarbamate was obtained (38 $\mathrm{mg}, 54.4 \mu \mathrm{moles})$ as a pink oil. $\left(\mathrm{R}_{\mathrm{f}}=0.55,50: 50\right.$ cyclohexane: ethyl acetate $)$

The BOC protective group was released from the aliphatic amine by addition of $20 \%$ fuming $\mathrm{HCl}$ in ethyl acetate for $10 \mathrm{~min}$ at RT. The reaction was quenched with $\mathrm{NaOH}$ and extracted with $\mathrm{CH}_{2} \mathrm{Cl}_{2}$. The product was purified by creating a silica gel filter, impurities were removed with washes of cyclohexane: ethyl acetate (from $100 \%$ cyclohexane to $100 \%$ ethyl acetate) and then the product was obtained by eluting with DMF. Solvent was evaporated in a rotovap system and the final product was obtained as a pink/orange oil $\left(\mathrm{R}_{\mathrm{f}}=0.0,70: 30\right.$ cyclohexane: ethyl acetate; $20 \mathrm{mg}$, yield: $\left.33 \%\right)$. ${ }^{1} \mathrm{H}$ NMR $\left(400 \mathrm{MHz}, \mathrm{CDCl}_{3}\right) \delta 1.25$ (m, 1.94H, CH2-CH2-CH2), $1.51(\mathrm{~m}, 2.30 \mathrm{H}, \mathrm{CH} 2), 1.72(\mathrm{~m}, 2.07 \mathrm{H}, \mathrm{CH} 2)$, $2.13(s, 1.20 \mathrm{H}, \mathrm{CH} 3 \mathrm{ap}), 2.16$ (s, 1.18H, CH3 ap), 2.34 (t, 2.04H, NH-CO-CH2), $2.43(2 s, 2.66 \mathrm{H}$, $\mathrm{CH} 3 \mathrm{p}), 2.71$ (m, 2.00H, CH2-NH2), 6.85-7.70 (m, 6H, ArH), 8.18 (s, 0.35H, H-7 p), $8.22(s, 0.65 \mathrm{H}$, H-7 ap). [p:ap] conformers 40:60

\subsubsection{Synthesis of 8-amino- $\mathrm{N}-(3-(3,3,4,4,5,5$-hexafluoro-2-(2-methylbenzo[b]thiophen- 3-yl)cyclopent-1-enyl)-2-methylbenzo[b]thiophen-6-yl)octanamide}

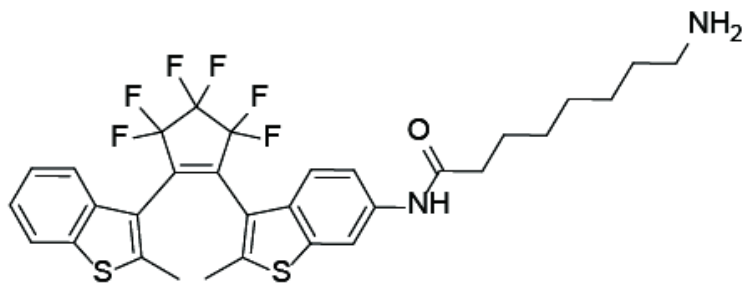

$\mathrm{PCaOcNH}_{2}$

A solution containing $\mathrm{PCmNH}_{2}(48 \mathrm{mg}, 100$ $\mu$ moles) was dissolved in dry $\mathrm{CHCl}_{3}$. The solution was added to a dry flask containing (9H-fluoren-9yl)methyl 8-chloro-8-oxooctylcarbamate $(72.0 \mathrm{mg}$, $180 \mu$ moles) and allowed to react at $30^{\circ} \mathrm{C}$ for $2 \mathrm{~h}$. The reaction was neutralized with $\mathrm{NaOH}$ and the crude product was extracted with $\mathrm{CH}_{2} \mathrm{Cl}_{2}$; a pink oil was obtained. Purification was performed on a silica gel column with cyclohexane: ethyl acetate (70:30) mobile phase. The intermediate product $(9 \mathrm{H}-$ fluoren-9-yl)methyl 8-(3-(3,3,4,4,5,5-hexafluoro-2-(2-methylbenzo[b]thiophen-3-yl)cyclopent-1enyl)-2-methylbenzo[b]thiophen-6-ylamino)-8-oxooctylcarbamate, PCaocFmoc, was obtained (61.8 mg, $73 \mu$ moles $)$ as a pink wax. $\left(\mathrm{R}_{\mathrm{f}}=0.40,60: 40\right.$ cyclohexane: ethyl acetate $)$

The Fmoc protective group was released from the aliphatic amine by addition of excess piperidine (650 $\mu 1,6.5$ mmoles) in $\mathrm{CHCl}_{3}$ at $50{ }^{\circ} \mathrm{C}$ for 20 minutes. The reaction was quenched with $\mathrm{HCl}$ and extracted with $\mathrm{CH}_{2} \mathrm{Cl}_{2}$. The product was purified by silica gel column, with cyclohexane: THF (30:70) mobile phase. Solvent was evaporated in a rotovap system and the final product was obtained as a pink-yellow oil $\left(\mathrm{R}_{\mathrm{f}}=0.0,70: 30\right.$ cyclohexane: ethyl acetate; $27.5 \mathrm{mg}$, yield: 44\%). ${ }^{1} \mathrm{H}$ NMR (400 MHz, $\left.\mathrm{CDCl}_{3}\right) \delta 1.33(\mathrm{~m}, 9.98 \mathrm{H}, \mathrm{CH} 2-\underline{\mathrm{CH}} 2-\mathrm{CH} 2), 1.51(\mathrm{~m}, 2.30 \mathrm{H}, \mathrm{CH} 2), 1.72(\mathrm{~m}, 2.07 \mathrm{H}, \mathrm{CH} 2), 2.13(\mathrm{~s}$, $1.70 \mathrm{H}, \mathrm{CH} 3 \mathrm{ap}), 2.16$ (s, 2.08H, CH3 ap), 2.34 (t, 2.26H, NH-CO-CH2), $2.43(2 s, 2.71 \mathrm{H}, \mathrm{CH} 3 \mathrm{p})$, $2.71(\mathrm{~m}, 2.00 \mathrm{H}, \underline{\mathrm{CH} 2}-\mathrm{NH} 2), 6.85-7.70(\mathrm{~m}, 6 \mathrm{H}, \mathrm{ArH}), 8.18(s, 0.42 \mathrm{H}, \mathrm{H}-7 \mathrm{p}), 8.22(s, 0.65 \mathrm{H}, \mathrm{H}-7 \mathrm{ap})$. [p:ap] conformers 40:60. 


\subsubsection{Synthesis of 12-amino-N-(3-(3,3,4,4,5,5-hexafluoro-2-(2-methylbenzo[b]thiophen- 3-yl)cyclopent-1-enyl)-2-methylbenzo[b]thiophen-6-yl)dodecanamide}

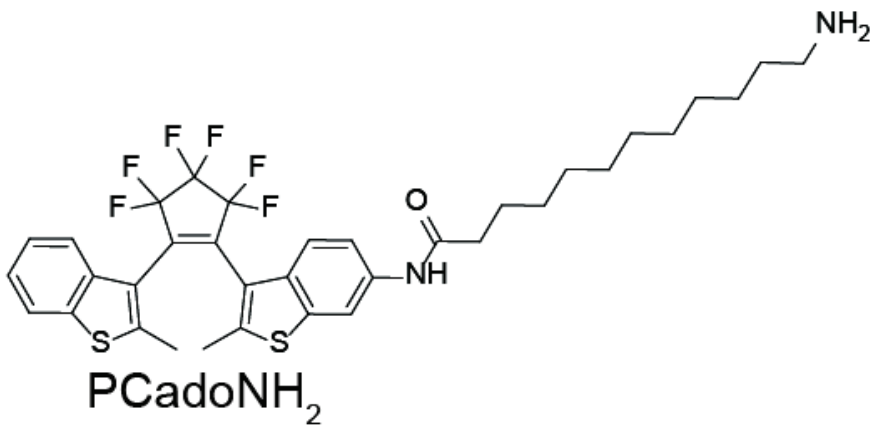

A solution containing $\mathrm{PCmNH}_{2}(48 \mathrm{mg}$, $100 \mu$ moles) was dissolved in dry $\mathrm{CHCl}_{3}$. The solution was added to a dry flask containing (9H-fluoren-9-yl)methyl 12chloro-12-oxododecylcarbamate $(82.1 \mathrm{mg}$, $180 \mu$ moles) and allowed to react at $30{ }^{\circ} \mathrm{C}$ for $2 \mathrm{~h}$. The reaction was neutralized with $\mathrm{NaOH}$ and the crude product was extracted with $\mathrm{CH}_{2} \mathrm{Cl}_{2}$; a pink oil was obtained. Purification was performed on a silica gel column with cyclohexane: ethyl acetate (70:30) mobile phase. The intermediate product (9H-fluoren-9-yl)methyl 12-(3-(3,3,4,4,5,5-hexafluoro-2-(2methylbenzo[b]thiophen-3-yl)cyclopent-1-enyl)-2-methylbenzo[b]thiophen-6-ylamino)-12oxododecylcarbamate, PCaocFmoc, was obtained $(40.6 \mathrm{mg}, 45 \mu$ moles $)$ as a golden oil. oil $\left(\mathrm{R}_{\mathrm{f}}=0.65\right.$, 60:40 cyclohexane: ethyl acetate)

The Fmoc protective group was released from the aliphatic amine by addition of excess piperidine (650 $\mu 1,6.5$ mmoles) in $\mathrm{CHCl}_{3}$ at $50{ }^{\circ} \mathrm{C}$ for 20 minutes. The reaction was quenched with $\mathrm{HCl}$ and extracted with $\mathrm{CH}_{2} \mathrm{Cl}_{2}$. The product was purified by silica gel column, with cyclohexane: THF (30:70) mobile phase. Solvent was evaporated in a rotovap system and the final product was obtained as a pink-yellow oil $\left(\mathrm{R}_{\mathrm{f}}=0.0,70: 30\right.$ cyclohexane: ethyl acetate; $18.3 \mathrm{mg}$, yield: $\left.27 \%\right) .{ }^{1} \mathrm{H}$ NMR $(400 \mathrm{MHz}$, $\left.\mathrm{CDCl}_{3}\right) \delta 1.27(\mathrm{~m}, 16.80 \mathrm{H}, \mathrm{CH} 2-\mathrm{CH} 2-\mathrm{CH} 2), 1.51(\mathrm{~m}, 2.30 \mathrm{H}, \mathrm{CH} 2), 1.72(\mathrm{~m}, 2.07 \mathrm{H}, \mathrm{CH} 2), 2.14(s$, $2.12 \mathrm{H}, \mathrm{CH} 3 \mathrm{ap}), 2.17$ (s, 2.22H, CH3 ap), 2.34 (t, 2.04H, NH-CO-CH2), $2.43(2 s, 2.66 \mathrm{H}, \mathrm{CH} 3 \mathrm{p})$, $2.71(\mathrm{~m}, 2.00 \mathrm{H}, \underline{\mathrm{CH} 2}-\mathrm{NH} 2), 6.85-7.70(\mathrm{~m}, 6 \mathrm{H}, \mathrm{ArH}), 8.18(s, 0.32 \mathrm{H}, \mathrm{H}-7 \mathrm{p}), 8.22(s, 0.52 \mathrm{H}, \mathrm{H}-7 \mathrm{ap})$. [p:ap] conformers 38:62.

\subsubsection{Synthesis of $\mathrm{N}-(3-(3,3,4,4,5,5-h$ exafluoro-2-(2-methylbenzo[b]thiophen-3- yl)cyclopent-1-enyl)-2-methylbenzo[b]thiophen-6-yl)-2-(3-(3-methyl-3H-diazirin-3- yl)propanamido)propanamide}

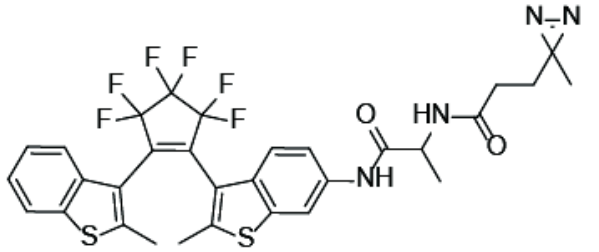

$\mathrm{PCalaNH}_{2}(22 \mathrm{mg}, 40 \mu$ moles) and SDA (Thermo\#26167, 11.2 $\mathrm{mg}, 50 \mu$ moles) were added to $4 \mathrm{ml}$ of dry $\mathrm{CHCl}_{3}$ and allowed to react for 90 minutes at RT. The flask was covered in aluminum foil to protect from light. The product was purified by silica gel column, with n-heptane: THF $(60: 40)$ mobile phase. The final product is yellow and crystalline $\left(\mathrm{R}_{\mathrm{f}}=0.30,1: 1\right.$ cyclohexane: THF; $23.9 \mathrm{mg}$, yield: $\left.90 \%\right) .{ }^{1} \mathrm{H}$ NMR $\left(400 \mathrm{MHz}, \mathrm{CDCl}_{3}\right) \delta$ $1.26(\mathrm{~d}, 2.91 \mathrm{H}, \underline{\mathrm{CH} 3}-\mathrm{CH}), 1.81$ (t, 1.87H, CO- $\underline{\mathrm{CH}} 2-\mathrm{CH} 2), 2.17$ (dd, 4.00H, Ar-CH3 ap), 2.28 (s, $3.90 \mathrm{H}, \mathrm{C}-\mathrm{CH} 3), 2.48$ (2s, 2.12H, Ar-CH3 p), 2.52 (t, 1.46H, CH2- $\underline{\mathrm{CH} 2}-\mathrm{C}), 4.71$ (m, 1.00, CH), 6.33 (m, 0.90H, CH-NH-CO), 7.07-7.72 (m, 6H, ArH), 8.13 ( $s, 0.43 \mathrm{H}, \mathrm{H}-7 \mathrm{ap}), 8.19(s, 0.25 \mathrm{H}, \mathrm{H}-7 \mathrm{p}), 8.78$ (s, 0.33H, Ar- $\underline{\mathrm{NH}}-\mathrm{CO}$ p), 8.93 (s, 0.55H, Ar- $\underline{\mathrm{NH}}-\mathrm{CO}$ ap). [p:ap] conformers 35:65. 


\subsubsection{Preparation of Photochromic Polymers with Aromatic Amine PC}

PMA (Sigma-531278, MW 6,000; $18.0 \mathrm{mg}, 3 \mu$ mole polymer, $120 \mu$ moles monomer) was added to a dry glass flask. $\mathrm{PCmNH}_{2}(25.0 \mathrm{mg}, 52 \mu$ moles$)$ was dissolved in anhydrous THF (5.5 ml) and added to the PMA in the flask. The flask was sonicated for 2 minutes and left to react at $50{ }^{\circ} \mathrm{C}$ with stirring. The solvent was reduced to approximately half and $5.55 \mathrm{mg}$ of dodecylamine ( $30 \mu \mathrm{moles})$ was added to the flask and the reaction was left overnight at $60{ }^{\circ} \mathrm{C}$. An additional $11 \mathrm{mg}(60 \mu$ moles $)$ of dodecylamine was added and allowed to react for $6 \mathrm{~h}$. A size-exclusion column of $\mathrm{LH}-20$ suspended in $\mathrm{CHCl}_{3}$ was utilized to separate unconjugated reagents. The final polymer PMA 6PC $75 \mathrm{C} 12$ is obtained as a dry film, for different $\mathrm{PCmNH}_{2}$ or dodecylamine concentrations the amount of reagent must be adjusted.

\subsubsection{Preparation of Photochromic Polymers with Primary Amine PC}

The ensuing methodology is for obtaining PMA 8 PCaoc $75 \mathrm{C} 12$, the methodology is valid for the other polymers adjusting for the desired concentrations and linker length. PMA $(6.0 \mathrm{mg}, 1 \mu$ mole polymer, $40 \mu$ moles monomer ) was added to a dry glass flask. $\mathrm{PCaocNH}_{2}(3.75 \mathrm{mg}, 6 \mu$ moles $)$ is prepared in anhydrous THF $(1.0 \mathrm{ml})$ and added to the PMA in the flask. The flask was sonicated for a minute and $2.8 \mathrm{mg}$ of dodecylamine $(15 \mu \mathrm{moles})$ was added to the flask and the reaction was left overnight at 60 ${ }^{\circ} \mathrm{C}$. An additional $2.8 \mathrm{mg}$ (15 $\mu$ moles $)$ of dodecylamine was added and allowed to react for $6 \mathrm{~h}$. A sizeexclusion column of $\mathrm{LH}-20$ suspended in $\mathrm{CHCl}_{3}$ was utilized to separate unconjugated reagents. After purification $10 \mathrm{mg}$ of dried polymer were obtained.

\subsubsection{Preparation of PMA 7PCahx 75C12 0.3AI}

PMA (6 mg, $1 \mu$ mole polymer, $40 \mu$ moles monomer) was introduced into a dry $10 \mathrm{ml}$ flask. Two $\mathrm{mL}$ of a DMF:DMSO solution of Alexa647 cadaverine (Invitrogen, A-30679; $0.16 \mu$ moles) was added. The solution was stirred at $60{ }^{\circ} \mathrm{C}$ for $90 \mathrm{~min}$ and then $7.7 \mathrm{mg}$ (14 $\mu$ moles) of $\mathrm{PCahxNH}_{2}$ was added in the minimal possible volume of THF. $1.85 \mathrm{mg}$ of dodecylamine $(10 \mu$ moles $)$ was added to the flask and the reaction was left overnight at $60^{\circ} \mathrm{C}$. An additional $3.7 \mathrm{mg}(20 \mu$ moles $)$ of dodecylamine was added and allowed to react for $6 \mathrm{~h}$. A size-exclusion column of LH-20 suspended in $\mathrm{CHCl}_{3}$ was utilized to separate unconjugated reagents. The final product is a reddish/purple solid (10 mg).

\subsubsection{Preparation of PMA 9PCala 75C12 2.5LY}

PMA ( $6 \mathrm{mg}, 1 \mu$ mole polymer, $40 \mu$ moles monomer) was introduced into a dry $10 \mathrm{ml}$ flask. A solution of lucifer yellow cadaverine (Molecular Probes, CAS \#149733-79-9, $10 \mu$ moles) in DMF (with trace DMSO) was added. The solution was stirred at $60{ }^{\circ} \mathrm{C}$ for $90 \mathrm{~min}$ and then $9 \mathrm{mg}$ (16 $\mu$ moles) of $\mathrm{PCalaNH}_{2}$ was added in the minimal possible volume of THF. Dodecylamine ( $2.8 \mathrm{mg}, 15 \mu$ moles) was added to the flask and the reaction was left overnight at $60{ }^{\circ} \mathrm{C}$. An additional $2.8 \mathrm{mg}$ (15 $\mu$ moles) of dodecylamine was added and allowed to react for $6 \mathrm{~h}$. A size-exclusion column of LH-20 suspended in $\mathrm{CHCl}_{3}$ was utilized to separate unconjugated reagents. The final product is a yellow/orange solid (11 $\mathrm{mg})$. 


\subsubsection{Preparation of PMA 6PCala 75C12 1.3PEG-Bt}

PMA (12 mg, $2 \mu$ mole polymer, $\sim 80 \mu$ moles monomer) was introduced into a dry $10 \mathrm{ml}$ flask. $\mathrm{PCalaNH}_{2}$ (4.2 mg, 7.6 moles) and Amine-PEG1 1-Biotin (Pierce\#26136, $0.92 \mathrm{mg}, 1.2 \mu$ moles) were added to the first flask as THF solutions. After one hour at $65^{\circ} \mathrm{C}$, dodecylamine (7.4 mg, $40 \mu$ moles) was added to the flask and the reaction was left overnight at $60{ }^{\circ} \mathrm{C}$. An additional $3.7 \mathrm{mg}(20 \mu$ moles $)$ of dodecylamine was added and allowed to react for $6 \mathrm{~h}$. A size-exclusion column of LH-20 suspended in $\mathrm{CHCl}_{3}$ was utilized to separate unconjugated reagents, $20 \mathrm{mg}$ of the final polymer were obtained.

\subsection{Measurements}

\subsubsection{Absorbance Spectroscopy.}

Absorbance spectra (300-800 nm) were acquired on a Cary $100 \mathrm{UV}-\mathrm{V}$ is spectrophotometer (Varian) utilizing $100 \mu \mathrm{L}$ microcuvettes with a $10 \mathrm{~mm}$ optical path. A solvent spectrum was utilized as a blank.

\subsubsection{Steady-State Fluorescence Spectroscopy}

Fluorescence spectra were acquired at $20{ }^{\circ} \mathrm{C}$ on a Cary Eclipse fluorescence spectrophotometer (Varian) utilizing Hellma $100 \mu \mathrm{L}$ microcuvettes. Fluorescence quantum yields were determined utilizing known standards (Rhodamine $6 \mathrm{G}$ in $\mathrm{EtOH}$ ) or QDs with the values provided by the manufacturers.

\subsubsection{Fluorescence Decay and Anisotropy Spectroscopy}

Fluorescence lifetime measurements were performed in a Fluoro- Log-TCSPC (Horiba Jobin Yvon). For observing LY, the excitation was carried out with a nanoLED N-460 source (Horiba Scientific) with the emission monochromator set to $525 \mathrm{~nm}$, a time-to-amplitude converter (TAC) range of $50 \mathrm{~ns}$, a pulse repetition frequency of $1 \mathrm{MHz}$, and a 5,000 counts peak value for automatic timing. For anisotropy measurements polarizers were introduced into the excitation and emission paths. In the case of $635 \mathrm{~nm}$ QD emission (Chapter 2), excitation was carried out with a N-460 nanoLED, and the emission monochromator was set to the peak at $635 \mathrm{~nm}$, a TAC range of $500 \mathrm{~ns}$, a pulse repetition frequency of $500 \mathrm{kHz}$, and 10,000 counts peak value for automatic timing. For $550 \mathrm{~nm}$ emitting QDs (Chapter 3) excitation was carried out with a nanoLED N-320 all other conditions were the same.

\subsubsection{TEM}

Samples of $0.1 \mu \mathrm{M}$ concentration were prepared in $50 \mathrm{mM}$ SBB buffer, placed on a carbon grid, and imaged with a Philips $120 \mathrm{kV}$ BioTwin microscope equipped with a $1024 \times 1024$ pixel GATAN CCD camera (Gatan, Inc.); 75-100 particles were measured to calculate diameter statistics. 


\subsubsection{Hydrodynamic Diameter}

Dynamic Light Scattering (DLS) measurements were made with a Nano-ZS Zetasizer nanoseries (Malvern Instruments) and a 90Plus Particle Size Analyzer (Brookhaven Instruments). Nanoparticle tracking measurements were made on a Nanosight LM10 (NanoSight Limited). Solutions were filtered (when appropriate) with $0.2 \mu \mathrm{m}$ filters and dilutions made with deionized water or buffer solution so as to achieve an optimal concentration.

\subsubsection{NMR}

Nuclear Magnetic resonance spectra were acquired on Bruker $400 \mathrm{MHz}$ (MPI bpc, Dep. of NMR based Structural Biology) in deuterated solvents (most often $\mathrm{CDCl}_{3}$ ). Spectra analysis and assignment was realized with MestReNova 7.0.2 (Mestrelab Research S.L.). Assignment was based on correlation with existing structures in the literature. Values reported in ppm based on TMS or non-deuterated solvent references.

\subsection{7 i-PrOH Titrations}

The titrations were realized on $100 \mu \mathrm{L}$ of sample in microcuvettes with serial additions of $i$-PrOH. After each addition the sample was mixed by pipetting and absorbance and fluorescence spectra were measured.

\subsection{Procedures}

\subsubsection{Silica Gel Chromatography}

Thin layer chromatography was realized on TLC Silica Gel 60 F254 Aluminum Sheets (Merck) with analytical grade solvents. A UV lamp was utilized to reveal the stains. Silica column purifications were realized with Silica Gel, 130-270 mesh, 60^ for column chromatography (Sigma-Aldrich) with analytical grade solvents.

\subsubsection{Size Exclusion Chromatography}

A column was prepared with Sephadex $\mathrm{LH}-20$, previously equilibrated in $\mathrm{CHCl}_{3}$. The separation medium will float in $\mathrm{CHCl}_{3}$ if allowed to settle, to avoid this, a thin layer of Sea Sand (Merck) can be applied on top, though this is not necessary as the medium will flow with the solvent and eliminate holes if the flow is continuous. The polymer was dried and resuspended in anhydrous $\mathrm{CHCl}_{3}$ and purified from unreacted reagents. The purification was followed by thin layer chromatography.

\subsubsection{Polymer Coating of QDs}

QD samples were precipitated from solvent supplied by the manufacturer and resuspended in anhydrous $\mathrm{CHCl}_{3}$. A solution of the polymer was prepared in anhydrous $\mathrm{CHCl}_{3}$. The solutions were mixed in a glass flask in a $1 \mathrm{mg}$ polymer for $200 \mathrm{pmol}$ of QDs proportion. The solution was mixed at 
$65{ }^{\circ} \mathrm{C}$ for $2 \mathrm{~h}$. The solvent was evaporated slowly, and once the sample was dry, SBB $50 \mathrm{mM} \mathrm{pH} 10$ was added in excess. The samples were left overnight with mild stirring.

\subsection{4 psQD Purification}

Sample purification was realized by filtration with $0.2 \mu \mathrm{m}$ inorganic filters, after which the solutions were concentrated to the $1 \mu \mathrm{M}$ range using Amicon 100,000 $\mathrm{kDa}$ cutoff filters (Millipore) and $50 \mathrm{mM}$ SBB, pH 9.0. Injections of 20 or $50 \mu \mathrm{L}$ (depending on concentration) were applied to a Superdex 200 analytical column (GE Healthcare Life Science) in an HPLC system with UV-Vis detection. Elution was with $50 \mathrm{mM} \mathrm{SBB}$, pH 9, starting with a flow of $50 \mu \mathrm{L} / \mathrm{min}$ for $3 \mathrm{~min}$ and then $20 \mu \mathrm{L} / \mathrm{min}$. The average run time was $70 \mathrm{~min}$. The desired fraction was collected in the $45 \mathrm{~min}$ range with slight variations depending on the QD size. In some cases a second injection was required to obtain the desired purity.

\subsubsection{Sample Irradiation}

Samples were irradiated using an $\mathrm{Hg}$ arc lamp (SUV-DC, Lumatec, Deisenhofen, Germany) and filters (340 $\pm 10 \mathrm{~nm}, 365 \pm 10 \mathrm{~nm}$, and $545 \pm 10 \mathrm{~nm}$ ). Samples were placed in Hellma $10 \mathrm{~mm} \times 2 \mathrm{~mm}$ or 3 $\mathrm{mm} \times 3 \mathrm{~mm}$ microcuvettes, filled such that the entire sample was exposed to light, thereby minimizing inhomogeneities. The samples were kept dilute $(<0.1$ absorbance at $540 \mathrm{~nm}$ for all conversion rates) so as to avoid internal filter effects.

\subsubsection{Conjugation to psQDs}

psQDs were stored in $50 \mathrm{mM}$ SBB pH 9.0 buffer, and dyes or proteins were diluted in SBB pH 7.3 from concentrated DMF solution in the case of dyes. EDC and Sulfo-NHS solutions were freshly prepared as $1 \mathrm{M}$ solutions in SBB $\mathrm{pH}$ 7.3. The psQDs and conjugate target were mixed in the desired proportion. Successful conjugation required excess conjugate and high concentrations. EDC was added at $\sim 2000$ equivalents per conjugate. The reactions took place at room temperature for 10-30 minutes then quenched with 2-mercaptoethanol, after which the solution was dialyzed using Pierce Slide-A-lyzer Mini Dialysis units (10,000 MWCO) or purified through exclusion column once more.

\subsubsection{Agarose gels}

Functionalized psQDs were run on $8 \times 10 \mathrm{~cm} \mathrm{1 \%}$ agarose gels in 0,5 TBE (Tris/Borate/EDTA) buffer at $100 \mathrm{mV}$ for $60-90$ minutes. $^{5}$

\subsubsection{Fittings}

Data analysis was realized with laboratory programs implemented on Mathematica 8.0 (Wolfram Research). In the case of coupled differential equations (FRET model, Section 3.2) the FindMinimum routine of Mathematica was used to solve the interlaced modeled equations. 


\subsubsection{Data Analysis Confocal Scanning SPM}

\section{Area Scan Macro.}

1. An image scan was imported into ImageJ $1.44 \mathrm{p}$ and cropped to eliminate border effects.

2. Background was subtracted [Rolling Background 50.0 Pixel] and an image threshold was applied [Adjust Threshold: Brightness, Min $=0, \operatorname{Max}=40$, Filter=pass].

3. The Image was transformed into a Binary image and the program was asked to find all areas that represented individual QDs [Analyze Particles: size=4-infinity, circularity $=0.00-1.00]$. These areas were marked as ROI and the coordinates were saved.

4. The ROI masks were applied to the original image after cropping and background subtraction.

5. Measurements provided a list with the mean, max, and minimum intensity of each detected psQD along with the measured area.

Time-Trace Macro.

1. Individual time-traces are separated and a threshold is applied [ $=\mathrm{IF}(\mathrm{Cell}>60$, Cell, 0$)]$. A QD is considered $\mathrm{ON}$ if the intensity is above 60 counts and OFF if it is below.

2. An event is defined as when a QD switches from OFF to ON. The borders of detection $\left(\mathrm{t}_{0}\right.$ and $\left.\mathrm{t}_{\text {end }}\right)$ are considered as OFF $[=(\operatorname{IF}($ Cell $>0,1,0)) *(\operatorname{IF}(\{$ Cell- 1$\}=0,1,0))]$.

\subsubsection{Data Analysis TIRF SPM}

1. Each movie was imported as a stack of images and cropped to eliminate border effects.

2. Background was subtracted from each image [Rolling=25 stack].

3. A projection of the maximum intensity of each pixel throughout the stack was created $[Z$ Project: Start=1, Stop=99, projection=Max intensity].

4. A threshold was applied to the Max Intensity projection [Adjust Threshold: Brightness, Min $=50$, Max $=255$, Filter=pass].

5. The image created in 4 was analyzed to find all areas that represented individual QDs[Analyze Particles: size $=4$-infinity, circularity $=0.66-1.00]$. These areas were marked as ROI and the coordinates were saved.

6. The ROI masks were applied to the original stack after cropping and background subtraction (Step 2).

7. Each ROI provides a time trace [Select window $=1$, roiManager $=$ Measure, Next Slice $=+1$, repeat].

8. Individual time-traces are separated and a threshold is applied[ $=\mathrm{IF}(\mathrm{Cell}>60, \mathrm{Cell}, 0)]$.

9. An event is defined as when a QD switches from OFF to ON. The borders of detection ( $t_{0}$ and $\left.\mathrm{t}_{\text {end }}\right)$ are considered as OFF $[=(\operatorname{IF}($ Cell $>0,1,0)) *(\operatorname{IF}(\{$ Cell- 1$\}=0,1,0))]$.

\section{References}

1. Giordano, L. Síntesis De Diheteroariletenos Fotocrómicos Y Su Aplicación Como Aceptores En Transferencia De Energía. Universidad de Buenos Aires, Buenos Aires, 2006.

2. Kawai, S.; Nakashima, T.; Kutsunugi, Y.; Nakagawa, H.; Nakano, H.; Kawai, T. Photochromic Amorphous Molecular Materials Based on Dibenzothienylthiazole Structure. J. Mat. Chem. 2009, 19, 3606 .

3. Utsumi, H.; Nagahama, D.; Nakano, H.; Shirota, Y. Synthesis of a Novel Family of Photochromic Amorphous Molecular Materials Based on Dithienylethene, Their Photochromic Properties and Application for Dual Image Formation. J. Mat. Chem. 2002, 12, 2612-2619. 
4. Kim, E.; Kim, M.; Kim, K. Diarylethenes with Intramolecular Donor-Acceptor Structures for PhotoInduced Electrochemical Change. Tetrahedron 2006, 62, 6814-6821.

5. Sperling, R. A.; Liedl, T.; Duhr, S.; Kudera, S.; Zanella, M.; Lin, C. A. J.; Chang, W. H.; Braun, D.; Parak, W. J. Size Determination of (Bio)Conjugated Water-Soluble Colloidal Nanoparticles: A Comparison of Different Techniques. J. Phys. Chem. C 2007, 111, 11552-11559. 


\section{ACKNOWLEDGEMENTS}

This work would not have been possible without the help and support of so many people. I'd like to begin with perhaps the two most important and dedicate this thesis to them, Prof. Dr. Elizabeth Jares-Erijman, Eli, and Prof. Dr. Maria del Carmen Mühlmann, Mom. Two incredible scientists, but more importantly, women. My mother's passion for her work in research was inspiring and made me see a future in science. Greater still was her passion for her students, her love was never ending for her family, friends, and everyone in her lab. These same characteristics I could see in Eli and I quickly found a place I considered my second home. Eli you provided me innumerable opportunities. Your mind, heart, and door were always open. I have no doubt that without you I would not be where I am today.

Thank you Tom and Donna. You taught me so much and guided me throughout. You pushed me when I was stuck or stubborn and encouraged and motivated me when you saw me on the right track. My admiration for you both is immense, your knowledge and work ethic inspiring. Your warmth of spirit was exemplified when you invited me into your home a cold night four years ago when I was spending my first Thanksgiving alone. Tom the hours we spent together discussing science and life in general always left me a richer man. Thank you both.

I would also like to thank my lab mates. Back in Buenos Aires, Carla, Doris, Eldara, Flor, Jime, Jimena, Jessy, Julia, Lucia, Maria, Sole, Fede(short), Fede(tall), Fran, and Martin. Every time I went back I felt as if I never left, nor wanted to. To everyone here in Göttingen: Anya, Elisa, Kerstin, Luciana, Renate, Albert, Anthony, Claudio, Dima, Lisandro, Luis, Pieter, Vova, and Wouter. I could not have wished for a better environment. I enjoyed our time together in the lab, but even better was the time we spent together outside of it. Flori thanks for all the help and proofreading during the writing process. Special mention to Raghu and Michelle who were forced to share an office with me and are probably a little worse for the wear now.

Thanks to Prof. Stalke for taking on the responsibility of helping me finish. Thanks to everyone in the Stalke group for being friendly and helping me when the students got too frustrated with my German.

Thanks to Prof. Subramaniam and everyone in Twente, especially Niels for taking the time to work with me and chat for countless hours.

To Dietmar Riedel and Gudrun Heim for the TEM images, to the Departments of Physical Biochemistry (MPIbpc), NMR based Structural Biology (MPIbpc), Facility for Advanced Light Microscopy (MPIbpc), NMR-Service (GAUG), and Zentrale Analytik (GAUG) for access to equipment and measurements. To the DAAD, BMBF, and ANPCyT for financial support.

Thanks to Manfred Konrad and Oliver Wenger who selflessly helped me during crucial times. 
I would like to thank three guys who meant so much to me during this thesis. Eli's lab was like my second home and that was because you guys were like my brothers. When I started out in the lab, Guille and Johnny, you taught me the ropes. Guille you were the gold standard from the moment I stepped into the lab and my project would not have gotten off the ground without you. Johnny your enthusiasm was contagious, you gave me the courage to try things outside of my comfort zone and don't think I have forgotten the positions (including this one) I got based on your recommendation. If they were my older brothers then Julio you were my twin. We worked together, got lost along the way, found some answers, but mostly just found new questions.

Outside of science there are too many people to thank. To all my family and friends, thank you! You are my place in this world and I could not get by a day, let alone four years, without knowing you are there supporting me. A special mention to those who honored us with their visits Flor, Gaston, Susana, Barbie, Connie, Vivi, Cucu, Vicki, Elmar, Rosita, Guille, Pau, and Gismondi.

Elmar and Rosita you brought me into your family and gave me the most important thing I needed to get ahead these four years and the same could be said about Paula and Axel. Thank you for being in my life.

Fernando your intelligence and natural talent always kept me on my game. I know I have to give it my all just to keep up. I am very proud to call you my brother. Gracias a los abuelos. Son una fuente infinita de inspiración y los amo con todo mi corazón. Dad I could not ask for a better rolemodel. You gave me my work ethic and taught me that I should only be satisfied if I did the best job I could do. I am the man I am today thanks to you.

Mom I miss you and think about you every day. Germany, fluorescence, microscopy.....You would have loved this!!!

Romi there isn't an idea, image, or page that did not go through you. You were instrumental to my work, but more importantly to me. You are my perfect counterpart and not just because I am design blind, you are bright, caring, patient, and a tireless worker all things I needed during these 4 years. Thank you for always being by my side. I love you and never could have done it without you. 


\section{Curriculum Vitae}

Name: Sebastián Andrés Díaz

Birth date: January 29, 1983

Birthplace: Capital Federal, Argentina

Nationality: Argentina and USA

Diplom in Chemistry, FCEN, Universidad de Buenos Aires, 2007.

\section{Peer Reviewed Publications}

1) S. A. Díaz, G. O. Menendez, M. H. Etchehon, L. Giordano, T. M. Jovin and E. A. JaresErijman, "Photoswitchable Water-Soluble Quantum Dots: pcFRET Based on Amphiphilic Photochromic Polymer Coating" ACS Nano 2011, 5, 2795-2805.

2) S. A. Díaz, L. Giordano, T. M. Jovin and E. A. Jares-Erijman, "Modulation of a Photoswitchable Dual-Color Quantum Dot Containing a Photochromic FRET Acceptor and an Internal Standard" Nano Lett. 2012, 12, 3537-3544.

3) S. A. Díaz, L. Giordano, J. C. Azcárate, T. M. Jovin and E. A. Jares-Erijman, "Quantum Dots as Templates for Self-Assembly of Photoswitchable Polymers: Small, Dual-Color Nanoparticles Capable of Facile Photomodulation" J. Am. Chem. Soc. 2013, 135, 3208-3217.

4) F. Gillanders, L. Giordano, S. A. Diaz, T. M. Jovin and E. A. Jares-Erijman, "Photoswitchable Fluorescent Diheteroarylethenes. Substituent Effects on Photochromic and Solvatochromic Properties" In Preparation. 2013.

\section{Conference Proceedings Publications}

1) S. A. Díaz, A. M. Toscani, D. J. Arndt-Jovin, T. M. Jovin, E. A. Jares-Erijman, "Small Photostable Photoswitchable Quantum Dots as Nanotools for Live Cell Imaging" Proc. Nanotech 2011 Vol. 3 2011, 205-208.

\section{Oral Presentations at International Conferences}

1) S. Díaz and E.A. Jares-Erijman. "Quantum Dot Sensors and Nanoactuators for Living Cells" NaNaX 4, Munich / Tutzing, Germany. April 11-15, 2010.

2) S. Díaz, A. Toscani, D. Arndt-Jovin, T. Jovin, E. Jares-Erijman. "Small Photostable Photoswitchable Quantum Dots as Nanotools for Live Cell Imaging" Nanotech Conference and Expo, Boston, USA. June 13-16, 2011. 


\section{Poster Presentations (first author only)}

1) S. Díaz, D. Grumelli, M. Etchehon, E. Rodríguez-Cobo, and E.A. Jares-Erijman. "Synthesis and Characterization of Functionalized Amphiphilic Polymer Micelles with Photochromic Diheteroarylethenes" US-Argentina Workshop on Nanomaterials, San Carlos de Bariloche, Argentina. March 15-17, 2009.

2) S. Díaz, N. Zijlstra, C. Blum, V. Subramaniam, T. Jovin and E.A. Jares-Erijman. "Biocompatible Quantum Dots Photoswitchable by pcFRET. Single-Particle Characterization" International Bunsen Discussion Meeting: Förster Resonance Energy Transfer in Life Sciences, Göttingen, Germany. March 27-30, 2011.

3) S. Díaz, L. Giordano, T. Jovin and E.A. Jares-Erijman. "Development of Stable Photomodulatable Fluorescent Nanoprobes for Aqueous and Cellular Systems" XXIV UIPAC Symposium on Photochemistry, Coimbra, Portugal. July 15-20, 2012.

4) S. Díaz, L. Giordano, T. Jovin and E.A. Jares-Erijman. "Novel Photomodulatable Fluorescent Nanoprobes for Aqueous and Cellular Systems." Annual Meeting of the German Biophysical Society, Göttingen, Germany. September 23-26, 2012.

5) S. Díaz, L. Giordano, N. Zijlstra, C. Blum, V. Subramaniam, T. Jovin and E.A. Jares-Erijman. "Photoswitchable Fluorescent Nanoprobes for Aqueous and Cellular Systems" Downscaling Science: $1^{\text {st }}$ Herrenhausen Conference on Nanoscience, Hannover, Germany. December 1214, 2012.

6) S. Díaz, L. Giordano, N. Zijlstra, C. Blum, V. Subramaniam, D. Arndt-Jovin, T. Jovin and E.A. Jares-Erijman. "Imaging Photoswitchable Fluorescent Nanoprobes" $1^{\text {st }}$ International Symposium Nanoscale Photonic Imaging, Göttingen, Germany. April 4-5, 2013. 